\title{
The Effect of Cold Roller-Bending on the Mechanical \& Geometric Properties of Steel Hollow Sections
}

\author{
Richards, Jonathon K.
}

How to cite:

Richards, Jonathon K. (2019) The Effect of Cold Roller-Bending on the Mechanical \& Geometric Properties of Steel Hollow Sections. thesis, Swansea University.

http://cronfa.swan.ac.uk/Record/cronfa52473

Use policy:

This item is brought to you by Swansea University. Any person downloading material is agreeing to abide by the terms of the repository licence: copies of full text items may be used or reproduced in any format or medium, without prior permission for personal research or study, educational or non-commercial purposes only. The copyright for any work remains with the original author unless otherwise specified. The full-text must not be sold in any format or medium without the formal permission of the copyright holder. Permission for multiple reproductions should be obtained from the original author.

Authors are personally responsible for adhering to copyright and publisher restrictions when uploading content to the repository.

Please link to the metadata record in the Swansea University repository, Cronfa (link given in the citation reference above.)

http://www.swansea.ac.uk/library/researchsupport/ris-support/ 


\title{
School of Materials Engineering
}

\author{
EngD Thesis
}

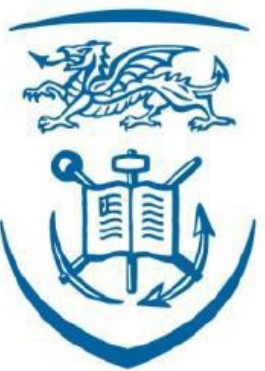

\section{Swansea University Prifysgol Abertawe}

\section{The Effect of Cold Roller-Bending on the} Mechanical \& Geometric Properties of

\section{Steel Hollow Sections.}

Candidate: Jonathon Richards BEng (Hons) MRes

Academic Supervisor: Dr A Das (Swansea University)

Industrial Supervisors: Dr C Owen \& Mr J Parley (TATA Steel)

Mr M Phillips (Angle Ring)

Sponsoring Companies: TATA Steel -Tubes

Angle Ring 


\section{Acknowledgements}

I would like to thank a number of people for their support and contributions to the project, either directly or indirectly. Firstly, I would like to express my gratitude to my project supervisors, Dr Chris Owen, Jim Parley, Dr Amit Das and Mark Phillips for encouraging me to pursue the project and have been helpful and attentive supervisors throughout. Their input has been invaluable.

I would like to thank the sponsors of my project for making it possible, Tata Steel Europe, Swansea University, Angle Ring Ltd, Specific and the welsh assembly government. I have very much enjoyed working with both industrial sponsors and look forward to working with them again in the future.

Most of all I would like to thank my family for supporting me and putting up with me through the process of writing up the thesis, it has been the hardest thing I have ever had to write.

Since starting this process, I have become married to the love of my life, had two wonderful children, started a new career with my sponsoring company and aged significantly!

I look forward to the next challenge life throws at me.

Jon Richards 


\begin{abstract}
In recent years there has been a significant increase in the use of steel hollow sections; in particular, where applications require a more aesthetic appeal. [1] As advances have been made in manufacturing and technology, the cost of hollow sections has decreased, however; it is still greater than that of hot-rolled open sections, such as Universal beams (I-beams). However, despite having a greater cost per tonne than open sections, hollow sections are being utilised increasingly due to a number of properties that make them an obvious choice for both aesthetically pleasing and utilitarian structures alike.
\end{abstract}

Tata Steel Tubes is one of Europe's leading manufacturers of welded steel tubes and pipes. They produce many thousands of tonnes of hot-finished and cold-formed tubes each year that are used in a diverse range of applications including the construction sector. Another key sector is energy \& power which relies heavily on conveyance tubes.

This project investigates the cold-roller-bending of plain carbon steel hollow sections for use in construction applications. The process of cold-roller-bending is widely considered to be an art rather than a science and therefore relatively little is known about the effects of the process on the physical and mechanical properties of the steel. In many cases it is assumed to be negligible[2] and therefore ignored by the standards and many of the guidance notes. Thus, the aim of this exercise was to determine and compare the effects of the cold bending process on the properties of hot-finished and cold-formed, plain-carbon steel, high-frequency induction (HFI) welded tubes.

There is no current EU, British or American standard that specifies changes in the mechanical properties brought about by the bending process or a maximum allowed curvature. This is because the properties are considered to largely remain the same as the original unbent material and that any alterations in these properties are made up for by the shape of the section.[3]-[5]

This project refutes this assumption. Hot-finished and cold formed hollow sections were curved using roller-bending equipment to a range of strains with increasing curvature.

The project has examined all of the requirements outlined by a structural engineering calculation, including the tensile strength, impact resistance, hardness and geometric resistances of the cross section. This has been performed in the hope of determining the most suitable section for the coldbending process.

The testing has determined that the process of cold-roller bending can be detrimental to many of the properties detailed above. All of the mechanical, mechanical and geometric properties examined in the experimentation were affected in some way. For example, the cross-sectional 
geometry of the section can be significantly altered by the bending process as the stresses involved distort the cross section of the tube. This leads to differences in the geometric properties when compared to the original unbent section. These properties include the sections resistance to bending and torsion. The tensile properties of the sections are also affected. During testing it was determined that cold-roller bending of steel leads to significantly reduced yield strength where the material is in compression during bending. This reduction in the elastic capacity of the section has been determined to have been caused by the Bauschinger effect; a phenomenon that occurs in many metals that leads to a reduction in capacity to resist stress and therefore yield stress of a section is reduced. The effect is observed when the section has underdone pre-strain beyond the materials yield point in the opposite direction to the current deformation. Further investigation around the Bauschinger effect revealed that it occurs when the section is first elongated and then compressed as well as where the section is compressed before elongation. For a number of applications, in Civil design, the plastic properties are not normally considered as a structure is not commonly designed to operate within its plastic region when in service. However, sections that have been curved using cold-roller bending will yield at significantly reduced stresses and therefore the plastic properties should be considered, particularly if the section is being used in a position where the bending loads are being reversed (from that of bending), for example, as an archway. Furthermore, this reduced capacity to resist bending will lead to spreading of the archway when loaded from above. This will lead to increased lateral load being exerted on a support structure which must be considered when designing a structure using curved steelwork.

It was observed that both the hot-finished and cold-formed sections experienced a similar reduction in the yield strength during tensile testing (at the intrados) however; hot-finished sections regained their properties with further cold deformation more rapidly than the equivalent cold-formed product. This observation, coupled with improved impact resistances and superior geometric properties lead to the conclusion that hot-finished sections are considered to be more suitable for the cold-roller bending process. 


\section{Table of Contents}

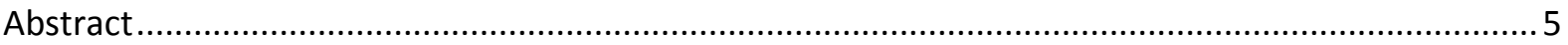

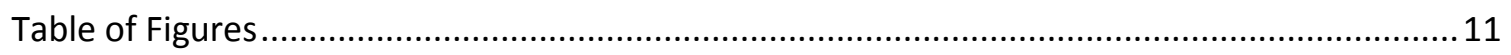

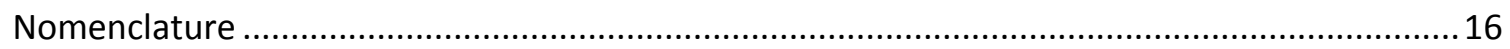

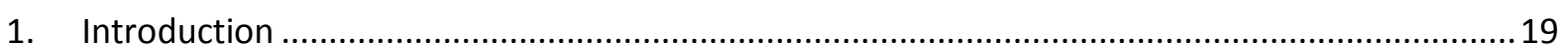

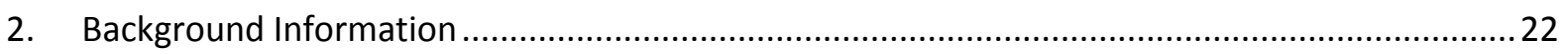

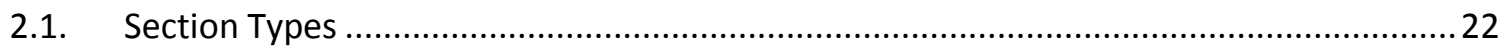

Structural High Frequency-Induction Welded (HFW) tubes \& Box sections (Hot-finished) ......... 22

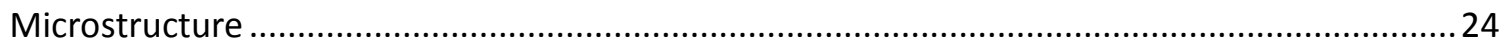

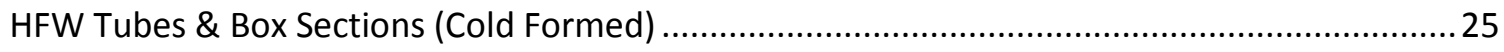

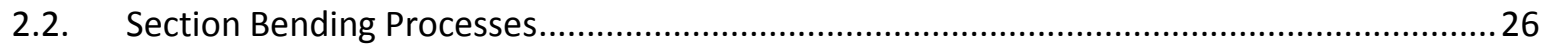

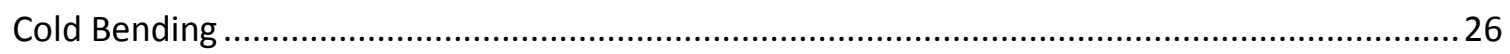

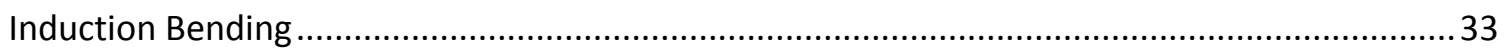

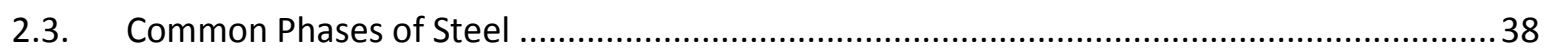

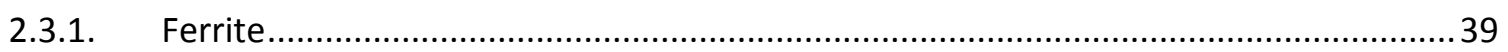

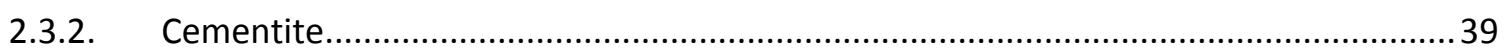

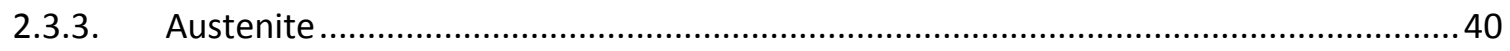

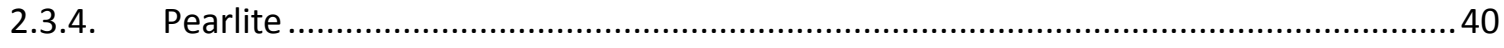

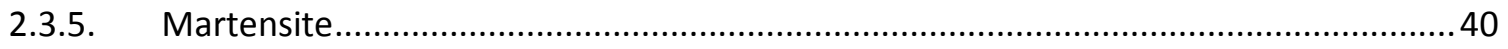

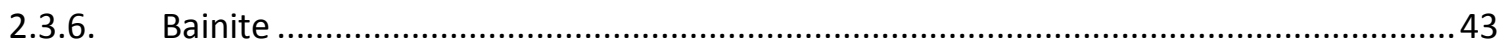

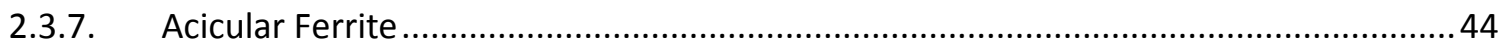

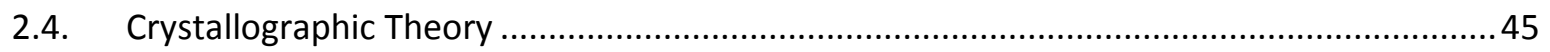

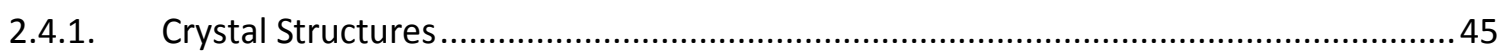

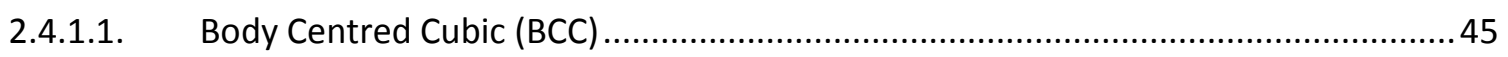

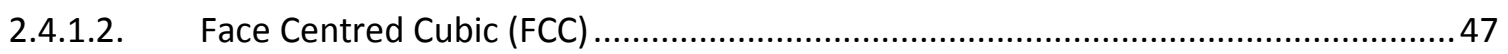

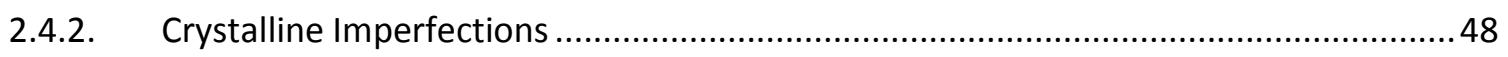

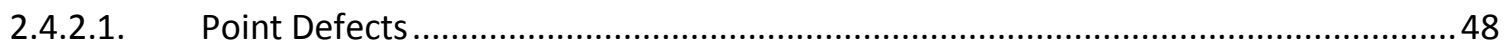

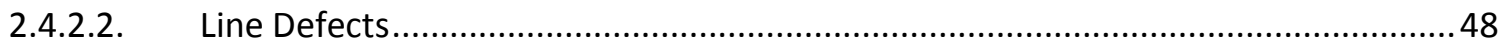

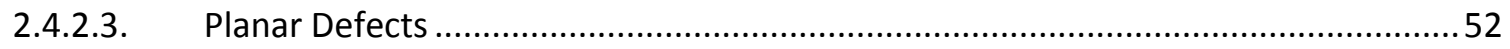

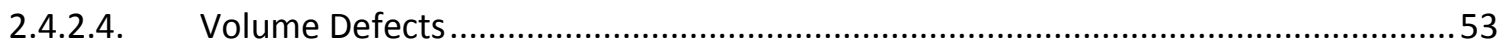

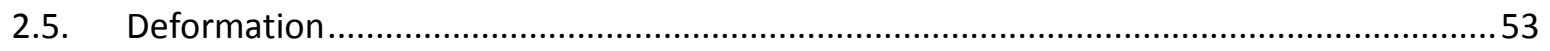

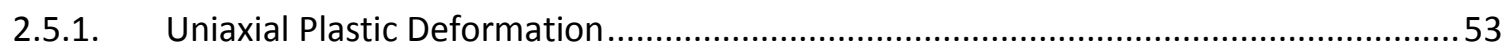

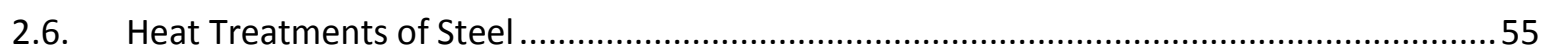

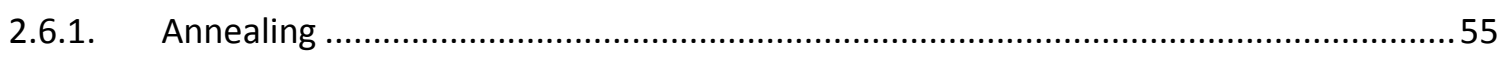




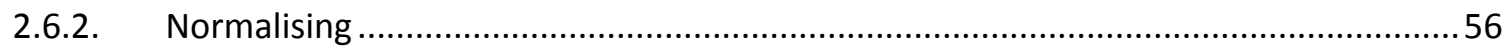

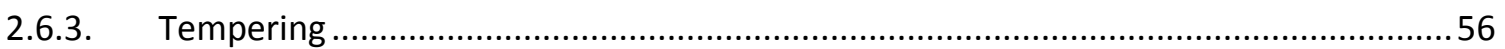

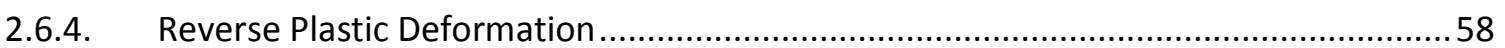

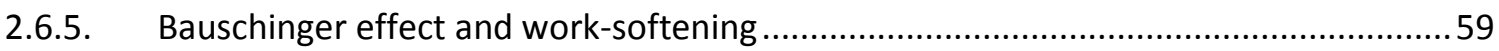

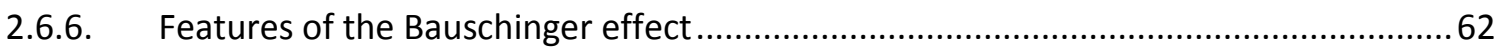

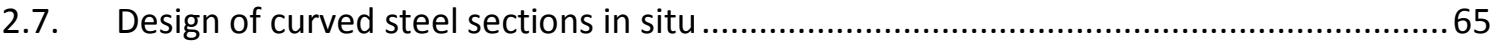

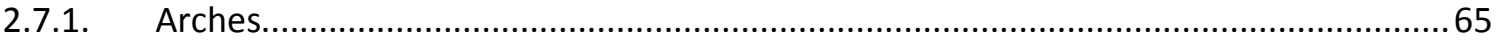

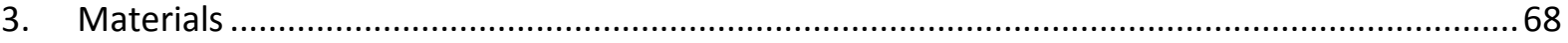

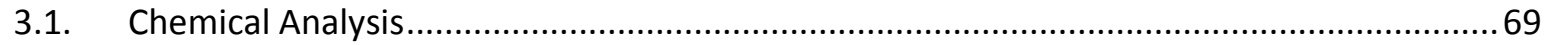

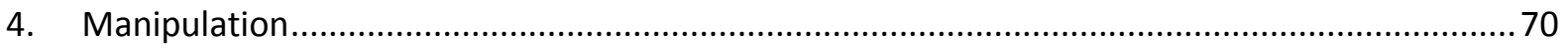

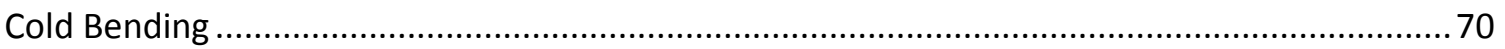

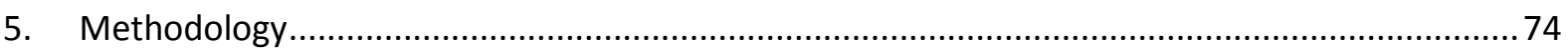

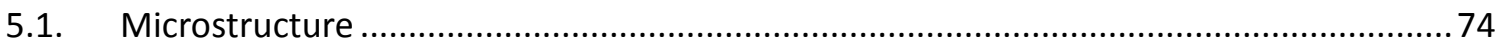

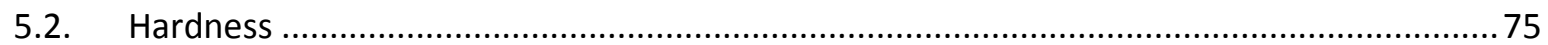

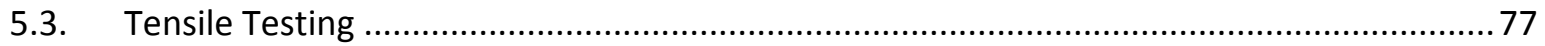

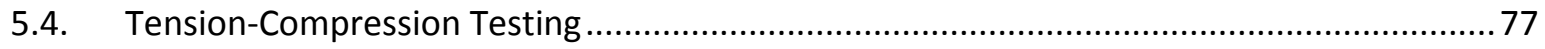

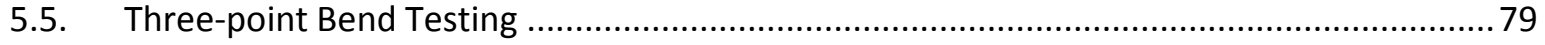

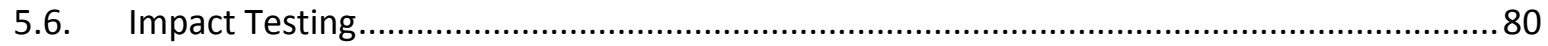

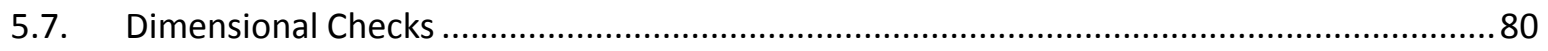

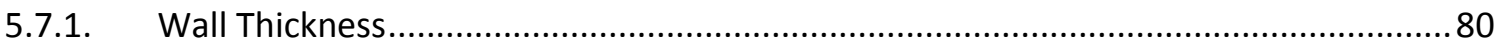

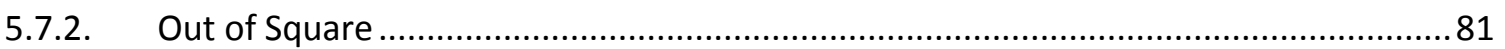

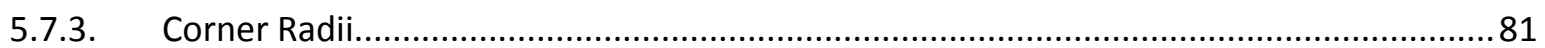

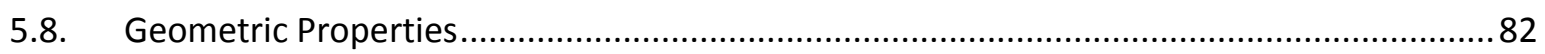

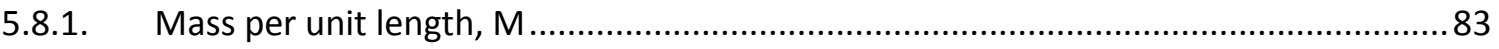

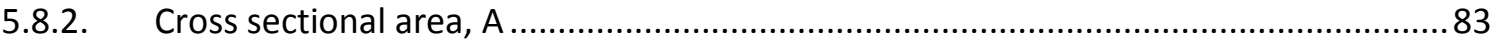

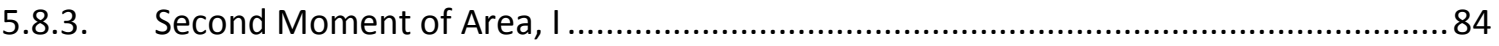

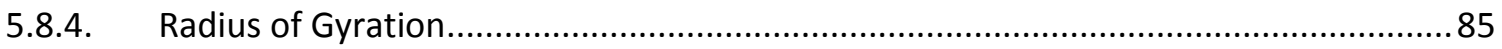

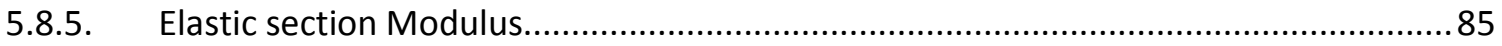

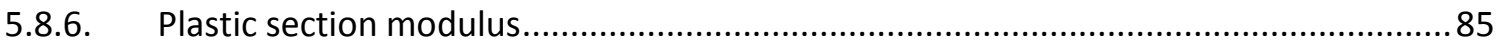

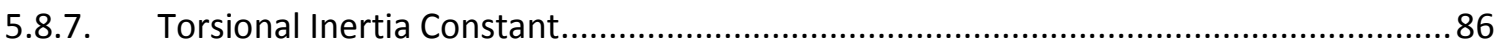

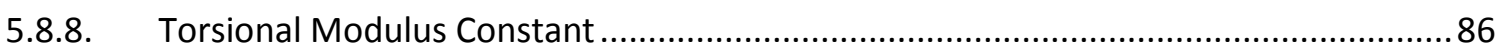

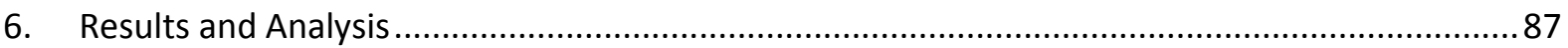

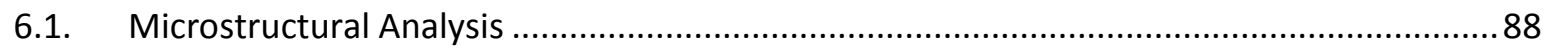

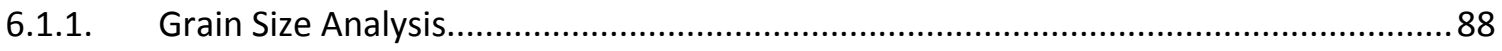


6.1.1.1. Hot-finished Rectangular Hollow Section Grain Size ............................................ 88

6.1.1.2. Cold-formed Rectangular Hollow Section Grain Size ............................................. 88

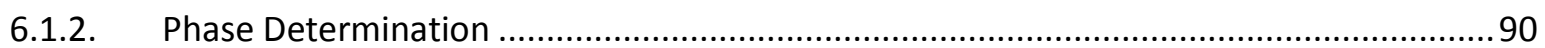

6.1.2.1. Hot-finished Square Hollow Section ................................................................. 90

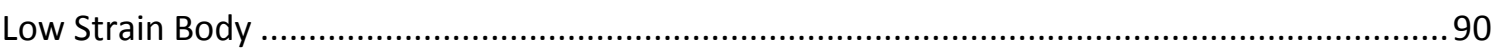

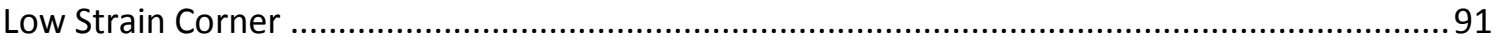

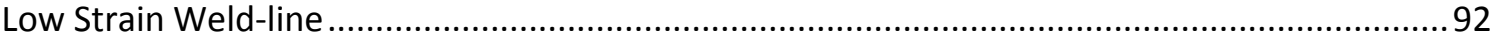

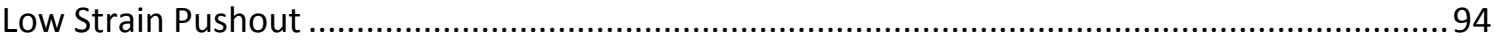

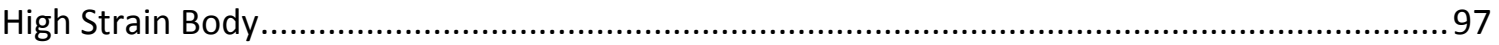

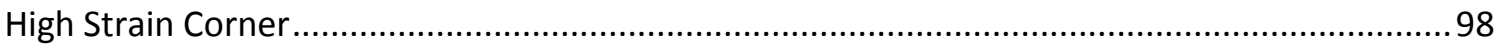

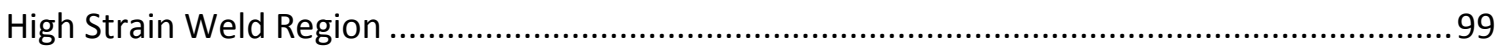

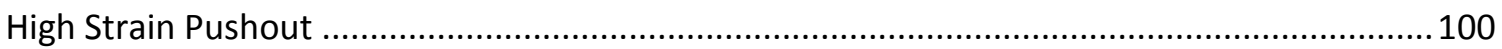

6.1.2.2. Cold-formed Square Hollow Section ................................................................. 103

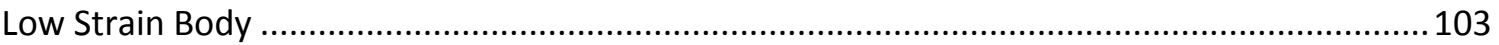

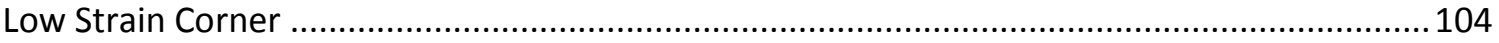

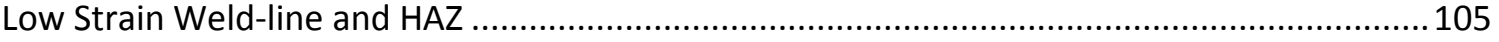

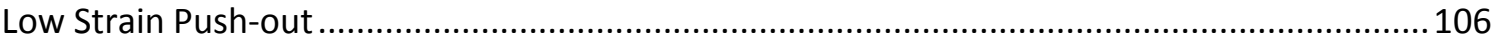

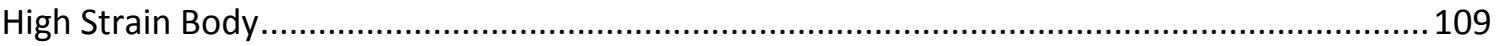

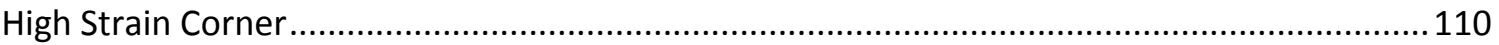

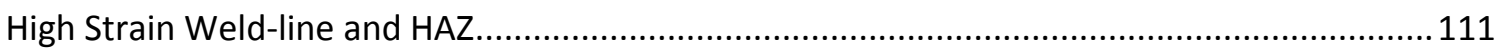

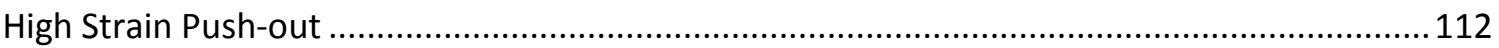

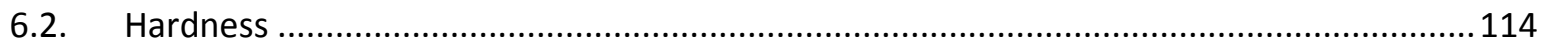

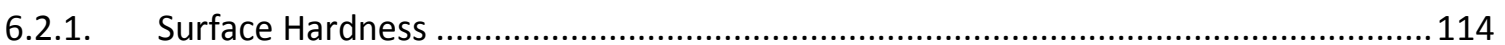

6.2.1.1. Hot-finished Rectangular Hollow Section ............................................................ 114

6.2.1.2. Cold-formed Rectangular Hollow Section .............................................................116

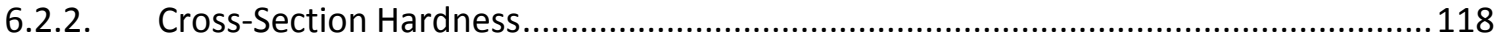

6.2.2.1. Hot-finished Rectangular Hollow Section ........................................................... 118

6.2.2.2. Cold-formed Square Hollow Section .................................................................... 123

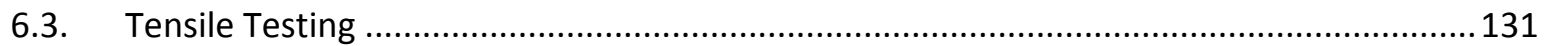

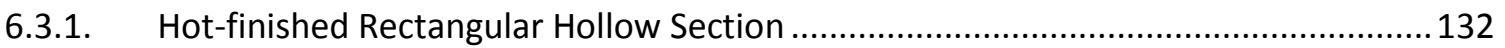

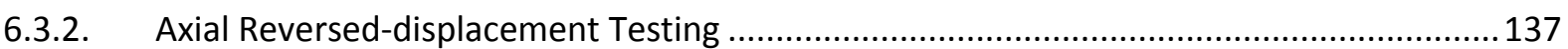

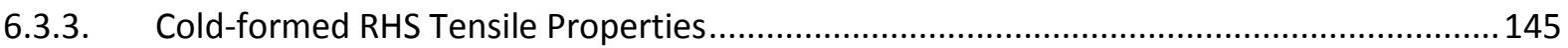

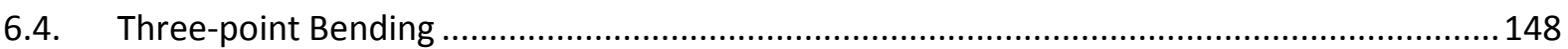

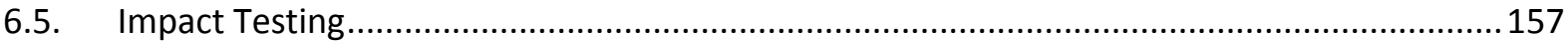


6.5.1. Hot-finished RHS Charpy Impact Resistance Properties ..........................................157

6.5.2. Cold-formed RHS Charpy Impact Resistance Properties ..............................................159

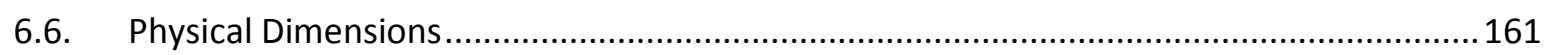

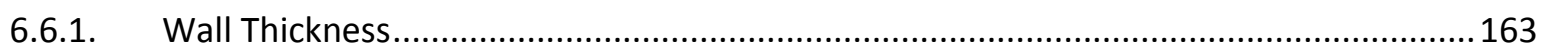

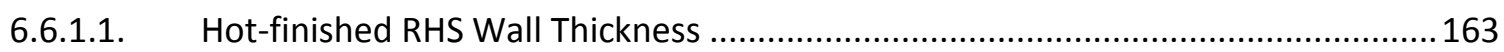

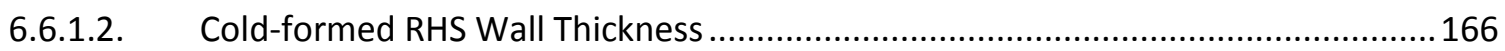

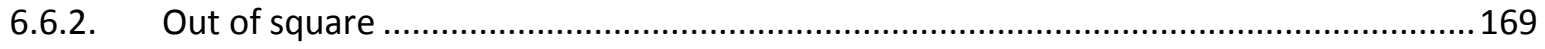

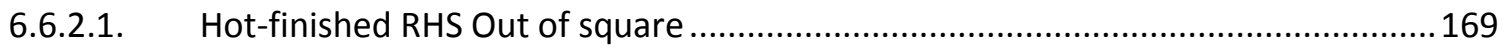

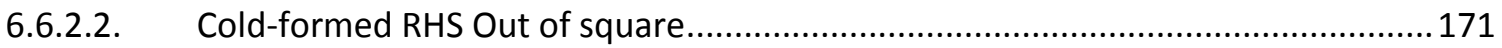

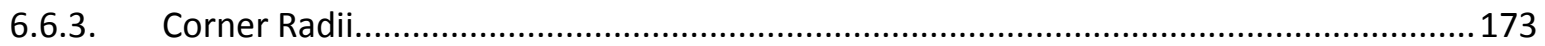

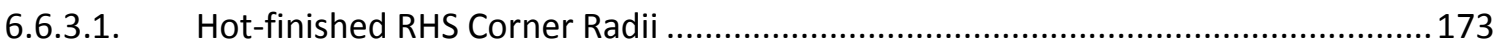

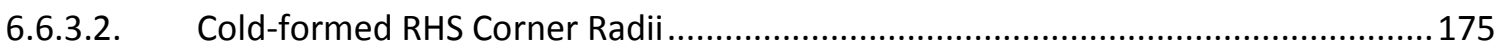

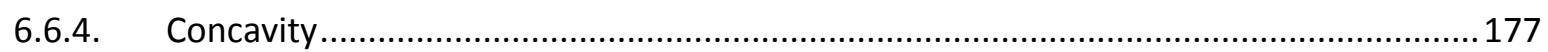

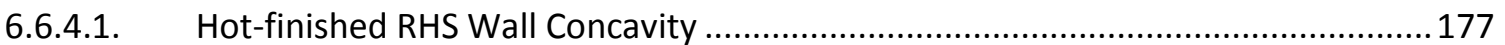

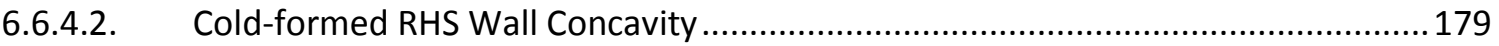

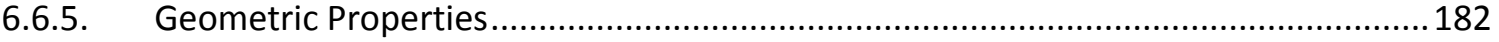

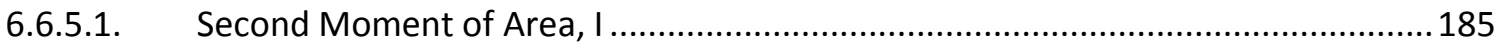

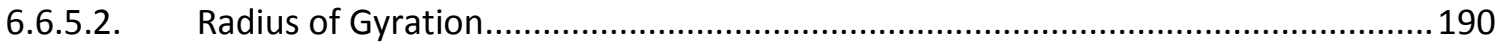

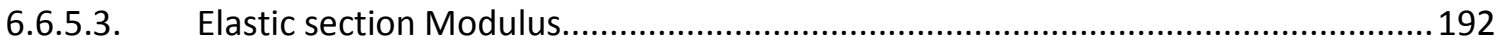

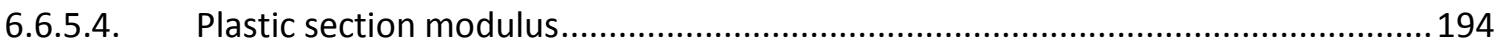

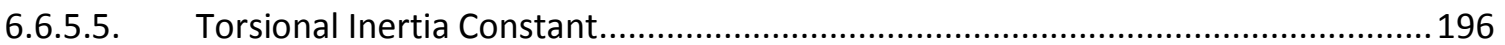

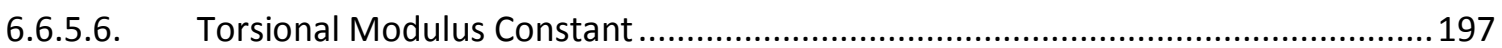

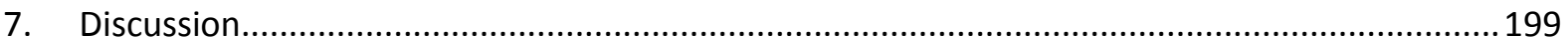

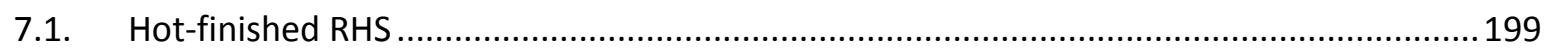

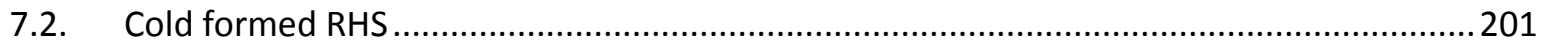

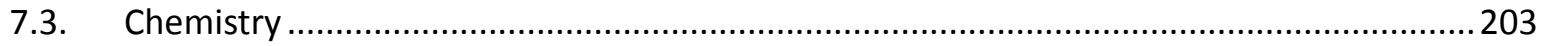

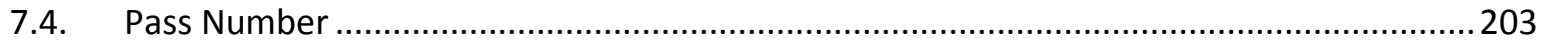

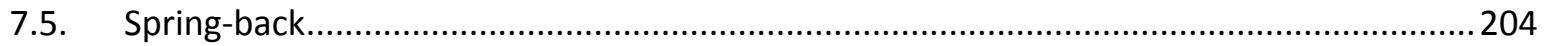

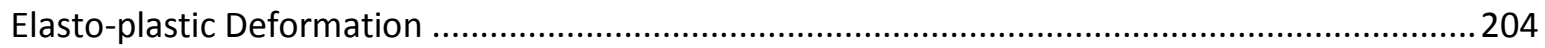

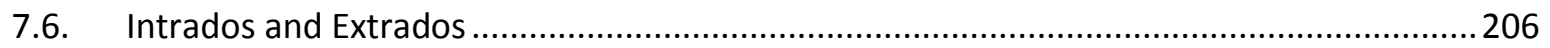

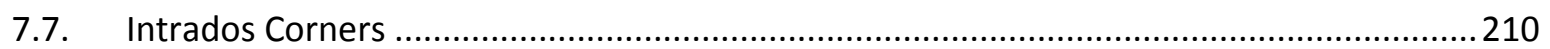

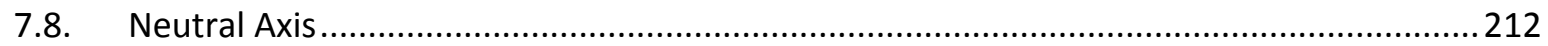

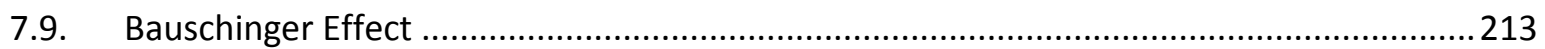

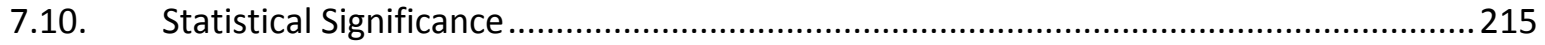


8. Conclusions

\section{Table of Figures}

Figure 1-Tube forming process (welded) [1] .....

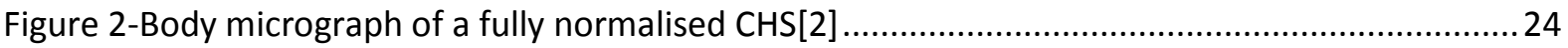

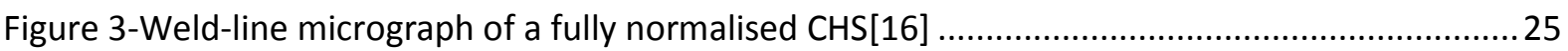

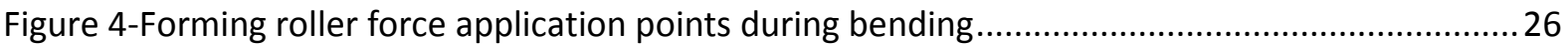

Figure 5-Global instability phenomena for arches that transfer loading to their abutments: (a) Snap

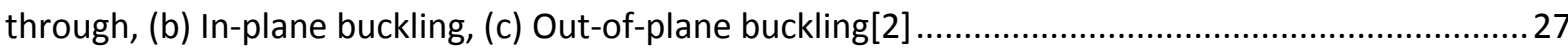

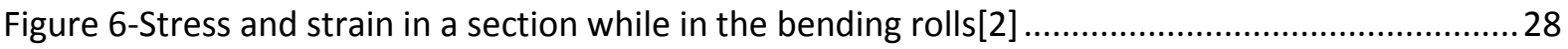

Figure 7-Stress distribution in the section during bending, spring-back stress distribution after load is removed and residual stress distribution in a section after bending where $\boldsymbol{p} \boldsymbol{y}$ is the yield stress ...... 29

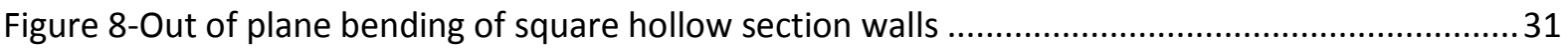

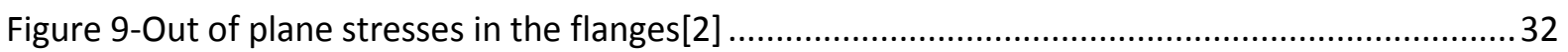

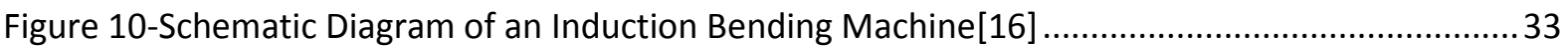

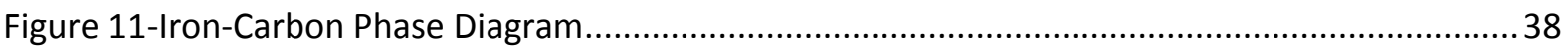

Figure 12-(left to right): Schematic BCC hard-sphere unit cell: Schematic BCC isolated unit cell:

Schematic diagram of an $\mathrm{BCC}$ isolated unit cell showing the relationship between the lattice constant, $\mathrm{a}$ and the atomic radius, $\mathrm{R}[35]$

Figure 13-(left to right): Schematic FCC hard-sphere unit cell[35]: Schematic FCC isolated unit cell[35]: Schematic diagram of an FCC isolated unit cell showing the relationship between the lattice constant, $\mathrm{a}$ and the atomic radius, $\mathrm{R}[35]$

Figure 14-Schematic showing positive edge dislocation of a crystal lattice at atomic resolution[37]. 49

Figure 15-Movement of an edge dislocation through a crystal lattice[63] ........................................ 49

Figure 16-Screw dislocation schematic of a crystal lattice at atomic resolution [63] ..........................51

Figure 17-Schematic diagram of the Bauschinger effect where $\boldsymbol{\sigma} \boldsymbol{p}=$ maximum pre-stress, $\boldsymbol{\sigma r}=$ the

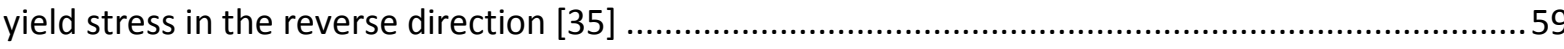

Figure 18-Modulus stress-strain curves

Figure 19-The Bauschinger effect parameters dependence on pre-strain for $0.17 \% \mathrm{C}$ steel derived by

Kostryzhev from work by Muir and Abel[10], [46]

Figure 20-Forward-reverse (modulus) stress-strain curve and its dependence on temperature for cold formed strip steel

Figure 21-Typical stress-strain curve for high and low carbon steels showing the relative decrease in yield for the reverse deformation phase[47].

Figure 22-Curved member acting as an arch exerting a lateral load on abutments as a vertical load is applied upon it[2]

Figure 23-Deflected shape of a curved member exerting pressure on supporting abutments as vertical load is applied[2]

Figure 24-The image shows the circular tube samples shortly after being cut into short lengths for transportation to their testing site at Swansea 
Figure 25-Schematic diagram showing the sampling position, number designation (1-6) and the bend direction (indicated with the blue arrow) of the rectangular hollow sections....................................72

Figure 26-Schematic diagram indicating the bending direction and the denoted axis ........................73

Figure 27-The image shows the hot formed tube samples that are to be used for microstructural

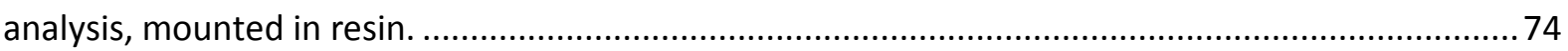

Figure 28 -Hot-finished sample bent to $0.25 \%$ strain intrados flange, magnification $500 \mathrm{x}-\mathrm{H} 0.25 \mathrm{H} 1 \ldots 90$ Figure 29-Hot-finished sample bent to $0.25 \%$ strain, intrados corner, magnification 500x-H0.25H4.91 Figure 30 -Hot-finished sample bent to $0.25 \%$ strain, intrados corner weld-line, magnification $25 \mathrm{x}$ $\mathrm{H} 0.25 \mathrm{H} 6$ .92

Figure 31 -Hot-finished sample bent to $0.25 \%$ strain, intrados corner weld-line, magnification $500 x$ $\mathrm{H} 0.25 \mathrm{H} 6$ 93

Figure 32 -Hot-finished sample bent to $0.25 \%$ strain, intrados corner weld-line pushout, magnification $25 \mathrm{x}$ H0.25H6.

Figure 33 -Hot-finished sample bent to $0.25 \%$ strain, intrados corner weld-line pushout, magnification $500 \mathrm{x}-\mathrm{H} 0.25 \mathrm{H} 6$

Figure 34 -Hot-finished sample bent to $0.25 \%$ strain intrados corner weld-line pushout, magnification

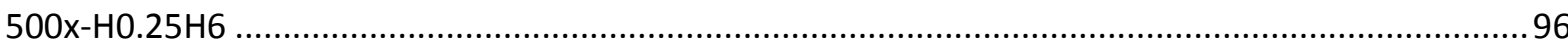

Figure 35 -Hot-finished sample bent to $5 \%$ strain, intrados flange, magnification 500x-H5.00L61..... 97 Figure 36-Hot-finished sample bent to $5 \%$ strain, extrados corner, magnification 500x-H5.00L65 ....98 Figure 37 -Hot-finished sample bent to $5 \%$ strain, intrados corner weld-line, magnification $500 x$ H5.00L66

Figure 38-Hot-finished sample bent to $5 \%$ strain, intrados corner weld-line push-out, magnification 25x-H5.00L66 100

Figure 39 -Hot-finished sample bent to $5 \%$ strain, intrados corner weld-line push-out, magnification 200x-H5.00L66 101

Figure 40 -Hot-finished sample bent to $5 \%$ strain, intrados corner weld-line push-out segregate void, magnification 500x-H5.00L66....

Figure 41 -Cold-formed sample bent to $0.25 \%$ strain, intrados flange, magnification 500x-C0.25H1 103 Figure 42 -Cold-formed sample bent to $0.25 \%$ strain, intrados corner, magnification 500x-C0.25H4104 Figure 43 -Cold-formed sample bent to $0.25 \%$ strain, neutral axis weld-line, magnification $500 x$ $\mathrm{C} 0.25 \mathrm{H} 2$ 105

Figure 44 -Cold-formed sample bent to $0.25 \%$ strain, neutral axis weld-line push-out, magnification $25 x-C 0.252$ 106

Figure 45 -Cold-formed sample bent to $0.25 \%$ strain, neutral axis weld-line push-out, magnification $500 \mathrm{x}-\mathrm{C} 0.25 \mathrm{H} 2$.

Figure 46 -Cold-formed sample bent to $0.25 \%$ strain, neutral axis weld-line push-out, magnification $500 \mathrm{x}-\mathrm{C} 0.25 \mathrm{H} 2$

Figure 47-Cold-formed sample bent to 5\% strain, intrados flange, magnification 500x-C5.00L31.... 109 Figure 48-Cold-formed sample bent to 5\% strain, intrados corner, magnification 500x-C5.00L34 ...110 Figure 49 -Cold-formed sample bent to $5 \%$ strain, neutral axis weld-line, magnification 500x-C5.00L32

Figure 50 -Cold-formed sample bent to $5 \%$ strain, neutral axis weld-line push-out, magnification $25 \mathrm{x}$ C5.00L32

Figure 51-Cold-formed sample bent to $5 \%$ strain, neutral axis weld-line pushout, magnification 500xC5.00L32 
Figure 52-Graph plotting the hot-finished RHS surface hardness for the intrados and extrados with increasing strain

Figure 53-Graph plotting the intrados and extrados surface hardness of the cold-formed RHS as strain is increased

Figure 54-Graph plotting high against low strain, hot-finished RHS cross-sectional hardness at the intrados surface

Figure 55-Graph plotting low strain, hot-finished RHS cross-sectional hardness at the intrados against the extrados surface

Figure 56-Graph plotting hot-finished RHS cross sectional hardness at the intrados and extrados low strain samples.

Figure 57-Graph plotting high strain, hot-finished RHS cross-sectional hardness at the intrados against the extrados.

Figure 58-Graph plotting high strain, hot-finished RHS cross-sectional hardness at the intrados corner against the extrados corner.

Figure 59-Graph plotting hot-finished RHS cross sectional hardness at the extrados of both high and low strain samples.

Figure 60-Graph plotting hot-finished RHS cross sectional hardness at the extrados corner of both high and low strain samples.

Figure 61-Graph plotting high strain, cold-formed RHS cross-sectional hardness at the intrados against the extrados.

Figure 62-Graph plotting high strain cold-formed RHS cross-sectional hardness at the intrados corner against the extrados corner.

Figure 63-Graph plotting, cold-formed RHS cross-sectional hardness at the intrados for both low and high strain samples.

Figure 64-Graph plotting, cold-formed RHS cross-sectional hardness at the intrados corner for both low and high strain samples.

Figure 65-Graph plotting, cold-formed RHS cross-sectional hardness at the extrados for both low and high strain samples.

Figure 66-Graph plotting, cold-formed RHS cross-sectional hardness at the intrados corner for both low and high strain samples.

Figure 67-Mean cross section Vickers hardness for the testing areas of the hot-finished RHS........129

Figure 68-Mean cross section Vickers hardness for the testing areas of the cold-formed RHS.........130 Figure 69-Graph plotting the difference in proof stress between the intrados and extrados of the hot-finished RHS with increasing strain

Figure 70-Graph plotting stress against displacement for hot-finished RHS.

Figure 71-Graph plotting the stress against strain for a compression-tension test carried out on hotfinished material

Figure 72-Graph plotting the modulus of stress against strain for a compression-tension test carried out on hot finished material, indicating the $0.2 \%$ proof stress ....

Figure 73-Graph plotting stress against strain for a tension-compression test carried out on hotfinished material-low strain

Figure 74-Graph plotting stress against strain for a tension-compression test carried out on hotfinished material-high strain. 
Figure 76-Graph plotting the stress and strain for a tension-compression test carried out on hotfinished material on the same axis

Figure 77-Graph plotting the stress and strain for a low strain tension-compression test performed on hot-finished material on the same axis

Figure 78-Graph plotting the difference in proof stress between the intrados and extrados of the cold-formed RHS with increasing strain.

Figure 79-Graph plotting stress against strain for hot-finished, low strain, three-point bend test and

$0.2 \%$ proof stresses

Figure 80-Graph plotting stress against strain for hot-finished, low-strain three-point bend test and $0.5 \%$ proof stresses

Figure 81-Graph plotting stress against strain for hot-finished, high-strain three-point bend test and $0.2 \%$ proof stresses

Figure 82-Graph plotting stress against strain for cold-formed, low strain three-point bend test and

$0.2 \%$ proof stresses

Figure 83-Graph plotting stress against strain for cold-formed, low strain three-point bend test and $0.5 \%$ proof stresses carried out

Figure 84-Graph plotting stress against strain for cold-formed, high-strain three-point bend test and

$0.2 \%$ proof stresses carried out . 155

Figure 85-Graph plotting mean Charpy impact resistance for the hot-finished material ...................160

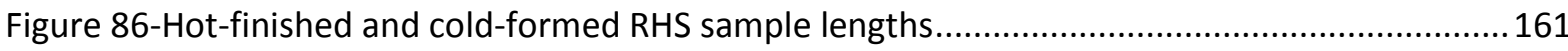

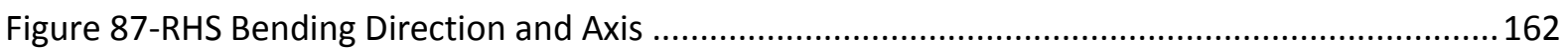

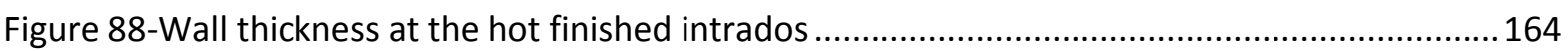

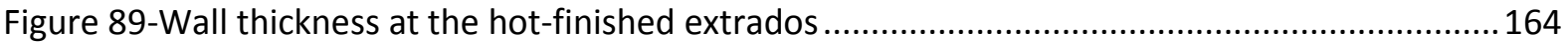

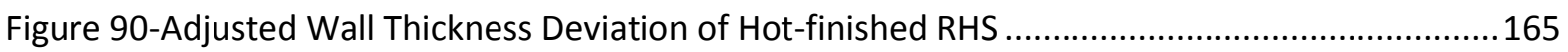

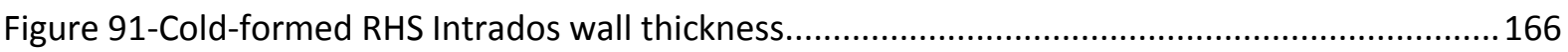

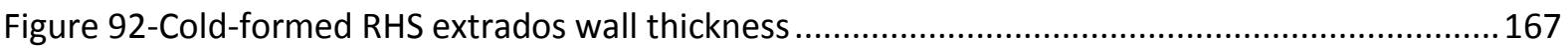

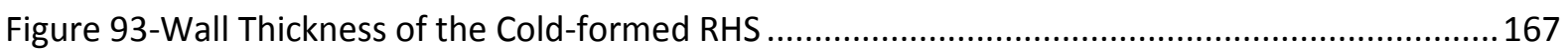

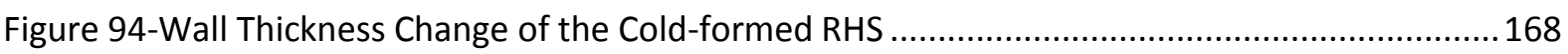

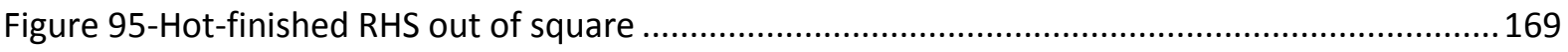

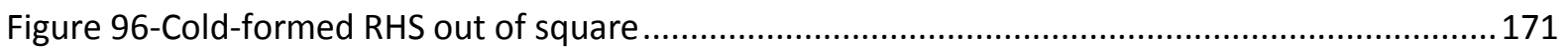

Figure 97-Hot-finished RHS percentage reduction in intrados corner radii ....................................173

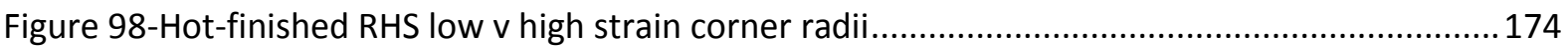

Figure 99-Cold-formed RHS Percentage Intrados Corner Radii Reduction........................................175

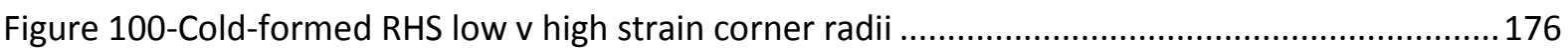

Figure 101-Intrados corner profile of a high strain cold-formed RHS ..........................................176

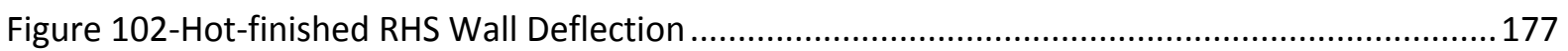

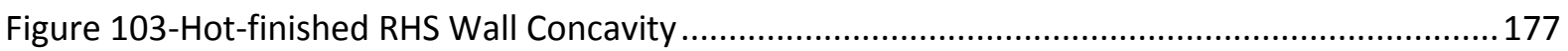

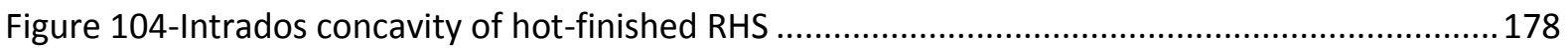

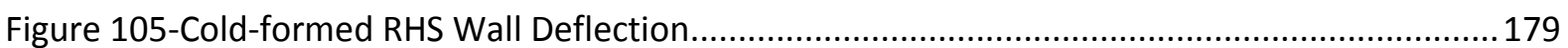

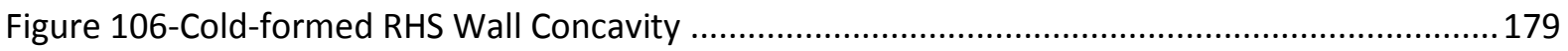

Figure 107-Intrados Concavity of a high strain cold-formed RHS................................................ 181

Figure 108-Geometry alteration in hot-finished RHS due to deformation........................................ 185

Figure 109-Geometry alteration in cold-formed RHS due to deformation ......................................185

Figure 110-Graph plotting the second moment of area, I as strain is increased for hot-finished RHS 
Figure 111-Graph plotting deviation in the second moment of area, I from that quoted in the specification as strain is increased for hot-finished RHS .

Figure 112-Graph plotting change in second moment of area, I as strain is increased for cold-formed RHS

Figure 113-Graph plotting deviation in the second moment of area, I from that quoted in the specification as strain is increased for cold-formed RHS

Figure 114-Graph plotting change in radius of gyration, $i$ as strain is increased for hot-finished RHS

Figure 115-Graph plotting change in radius of gyration, i as strain is increased for cold-formed RHS

Figure 116-Graph plotting change in elastic section modulus, $\mathbf{W e l}$ as strain is increased for hot-

finished RHS

Figure 117-Graph plotting change in elastic section modulus, $\mathbf{W e l}$ as strain is increased for coldformed RHS

Figure 118-Graph plotting change in plastic section modulus, $\boldsymbol{W p l}$ as strain is increased for hotfinished RHS

Figure 119-Graph plotting change in plastic section modulus, $\boldsymbol{W p l}$ as strain is increased for cold-

formed RHS

Figure 120-Graph plotting change in torsional inertia constant, $I t$ as strain is increased for hot-

finished RHS

Figure 121-Graph plotting change in torsional inertia constant $\boldsymbol{I t}$ as strain is increased for cold-

formed RHS

Figure 122-Graph plotting change in torsional modulus constant, $\boldsymbol{C t}$ as strain is increased for hot-

finished RHS

Figure 123-Graph plotting change in torsional modulus constant, $\boldsymbol{C t}$ as strain is increased for cold-

formed RHS.

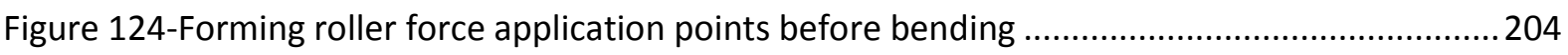

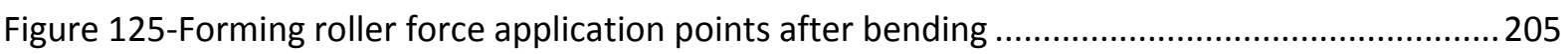

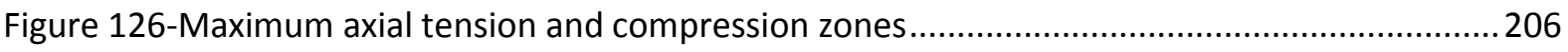

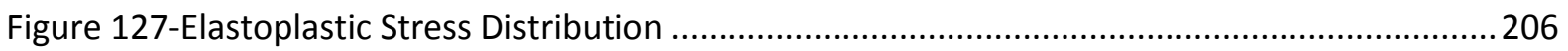

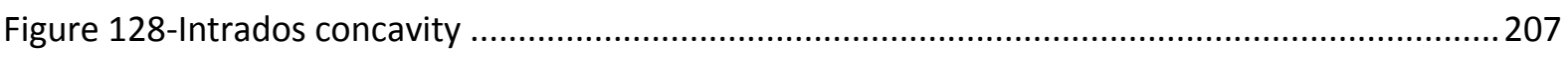

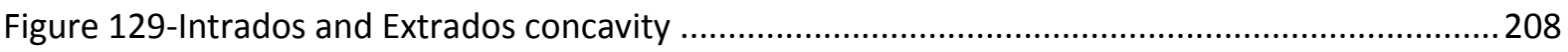

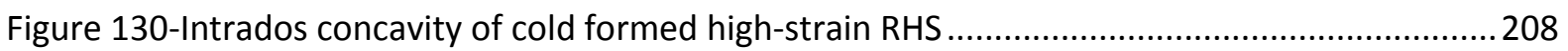

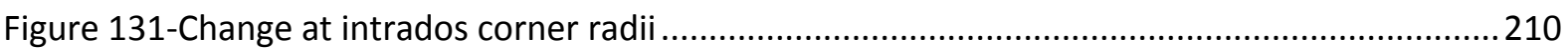

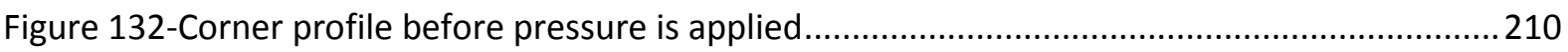

Figure 133-Elastoplastic stress distribution around the neutral axis .............................................212

Figure 134-lateral pressure experienced by the RHS during bending ..........................................212 
Nomenclature

\begin{tabular}{|c|c|}
\hline $\mathrm{HFI}$ & High Frequency Induction \\
\hline HFW & High Frequency Welded \\
\hline RHS & Rectangular Hollow Sections \\
\hline $\mathrm{CHS}$ & Circular Hollow Sections \\
\hline OD & Outer Diameter of a circular hollow section \\
\hline ID & Inner Diameter of a circular hollow section \\
\hline Intrados & Inside surface of a curved section \\
\hline Extrados & Exterior surface of a curved section \\
\hline AC & Alternating Current \\
\hline DC & Direct Current \\
\hline APF & Atomic Packing Factor \\
\hline BCC & Body Centred Cubic \\
\hline FCC & Face Centred Cubic \\
\hline $\mathrm{HCP}$ & Hexagonal Close Packed \\
\hline BCT & Body Centred Tetragonal \\
\hline APF & Atomic Packing Factor \\
\hline CEV & Carbon Equivalent Value \\
\hline LC & Low Carbon \\
\hline $\mathrm{HC}$ & High Carbon \\
\hline $\mathrm{V}_{\text {atoms }}$ & Volume of the atoms contained in a unit cell \\
\hline $\mathrm{V}_{\text {unit cell }}$ & Volume of a unit cell \\
\hline TMRC & Thermo-Mechanically Roller Cooled \\
\hline HV & Vickers Hardness (Unit) \\
\hline HAZ & Heat Affected Zone \\
\hline $\mathrm{R}$ & Radius \\
\hline $\mathrm{R}_{\text {in }}$ & Radius of the intrados \\
\hline $\mathrm{R}_{\mathrm{na}}$ & Radius of the neutral axis \\
\hline $\mathrm{S}_{\text {in }}$ & Length of intrados arc length \\
\hline $\mathrm{S}_{\mathrm{na}}$ & Length of neutral axis arc length \\
\hline wt\% & Weight percent \\
\hline $\mathrm{M}_{\mathrm{s}}$ & Martensite Start Temperature \\
\hline $\mathrm{M}_{\mathrm{F}}$ & Martensite Finish Temperature \\
\hline
\end{tabular}




\begin{tabular}{|c|c|}
\hline$\beta_{\sigma 1}$ & Bauschinger Stress Parameter 1-relative decrease in yield stress \\
\hline$\beta_{\sigma 2}$ & $\begin{array}{l}\text { Bauschinger Stress Parameter 2-Property restoration during reverse strain to } \\
0.2 \% \text { strain }\end{array}$ \\
\hline$\beta_{\sigma 3}$ & $\begin{array}{l}\text { Bauschinger Stress Parameter 3-Property restoration during reverse strain to } \\
0.5 \% \text { strain }\end{array}$ \\
\hline$\beta_{\sigma 4}$ & Bauschinger Stress Parameter 4-Property restoration during reverse strain \\
\hline$\sigma_{\mathrm{p}}$ & Max Pre-stress \\
\hline$\sigma_{\text {yield }} / \sigma_{\mathrm{y}} / \mathrm{p}_{\mathrm{y}} / \mathrm{f}_{\mathrm{y}}$ & Yield Stress \\
\hline$\varepsilon_{\text {yield }} / \varepsilon_{\mathrm{y}}$ & Yield Strain \\
\hline $\mathrm{p}_{\mathrm{yd}}$ & Design Yield Stress \\
\hline$\sigma_{\mathrm{r}}$ & Yield stress in reverse loading \\
\hline$\sigma_{\mathrm{r} 0.2}$ & Stress at $0.2 \%$ reverse strain \\
\hline$\sigma_{\mathrm{r} 0.5}$ & Stress at $0.5 \%$ reverse strain \\
\hline $\mathrm{E}_{\mathrm{p}}$ & Energy pent during pre-strain \\
\hline $\mathrm{E}_{\mathrm{r}}$ & Energy saved during reverse strain \\
\hline$\Delta \sigma_{\mathrm{s}}$ & Permanent Work Softening \\
\hline$\epsilon_{\mathrm{p}}$ & Pre-strain \\
\hline$\epsilon_{\mathrm{r}}$ & Reverse Strain \\
\hline$\beta_{\varepsilon}$ & Bauschinger Strain Parameter \\
\hline CMM & Coordinate Measuring Machine \\
\hline E & Young's Modulus \\
\hline $\mathrm{p}_{\mathrm{r}}$ & Residual stress at extreme fibres \\
\hline K & Strength Coefficient \\
\hline $\mathrm{M}_{\mathrm{p}}$ & Plastic Moment \\
\hline $\mathrm{M}_{\mathrm{sb}}$ & Spring-back Moment \\
\hline$S$ & Elastic section modulus \\
\hline Z & Plastic section modulus \\
\hline A & Cross sectional Area \\
\hline $\mathrm{T}$ & Section Gauge/Wall Thickness \\
\hline$\sigma_{1}$ & Longitudinal Bending Stresses \\
\hline$\sigma_{2}$ & Out of plane stresses \\
\hline$\tau$ & coexistent shear stress \\
\hline ASTM & American Society for Testing and Materials \\
\hline
\end{tabular}




\begin{tabular}{|c|l|}
\hline $\mathrm{I}$ & Second Moment of Area \\
\hline$\delta \mathrm{l}$ & Change in length \\
\hline $\mathrm{Ag}$ & Outside corner radius \\
\hline $\mathrm{A}_{\xi}$ & Inside corner radius \\
\hline $\mathrm{h}_{\mathrm{gyy}}$ & Outside corner radius-vertical \\
\hline $\mathrm{h}_{\mathrm{gxx}}$ & Outside corner radius-horizontal \\
\hline $\mathrm{h}_{\mathrm{gyy}}$ & Inside corner radius-vertical \\
\hline $\mathrm{h}_{\mathrm{gxx}}$ & Inside corner radius-horizontal \\
\hline$i_{y y}$ & Radius of Gyration \\
\hline $\mathrm{W}_{\mathrm{el} \mathrm{yy}}$ & Elastic Section Modulus-Vertical \\
\hline $\mathrm{W}_{\mathrm{el} \mathrm{xx}}$ & Elastic Section Modulus-Horizontal \\
\hline $\mathrm{W}_{\mathrm{pl} \mathrm{yy}}$ & Plastic Section Modulus-Vertical \\
\hline $\mathrm{W}_{\mathrm{pl} \mathrm{xx}}$ & Plastic Section Modulus-Horizontal \\
\hline $\mathrm{I}_{\mathrm{t}}$ & Torsional Inertia Constant \\
\hline$C$ & Torsional Constant \\
\hline$C_{t}$ & Torsional Modulus Constant \\
\hline
\end{tabular}




\section{Introduction}

In recent years there has been a significant increase in the use of steel hollow sections; in particular, where applications require a more aesthetic appeal. [1] As advances have been made in manufacturing and technology, the cost of hollow sections has decreased, however; it is still greater than that of hot-rolled open sections, such as Universal beams (I-beams). Hot-rolled open sections are more commonly used in industrial portal-frame structures, such as warehouses or farm sheds where large spans are required; they have the highest resistance to bending of any steel section in their working plane. However, despite having a greater cost per tonne than open sections, hollow sections are being utilised increasingly due to a number of properties that make them an obvious choice for both aesthetically pleasing and utilitarian structures alike.

Hollow sections have a very high torsional rigidity and, particularly in the cases of square and circular hollow sections, have no weak direction when considering bending resistance in either plane.[3], [6]-[9] These properties allow for more minimalistic lateral bracing systems, which often reduces the total mass of steel in the final superstructure. This also allows more windows to be installed where otherwise bracings would be required. Hollow sections also have a reduced area when surface coatings and fire protection are being considered (when compared to open sections). These combined factors allow an architect to produce a design for their client that is more aesthetically pleasing but with the same structural performance and comparable price to one constructed from open sections.

Tata Steel Tubes is one of Europe's leading manufacturers of welded steel tubes and pipes. They produce many thousands of tonnes of hot-finished and cold-formed tubes each year that are used in a diverse range of applications including the construction sector. Another key sector is energy \& power which relies heavily on conveyance tubes.

Both of these sectors rely increasingly on the use of curved steelwork, for example, the Wembley Stadium arch in London is formed from many curved steel tubes joined together to form the aesthetically pleasing superstructure. The oil and gas industry is also heavily reliant on curved steel tubes for pipeline and refinery use. The curving of steel may be performed using high temperature or cold forming techniques. Most structural sections are cold bent at ambient temperature to reduce cost, whereas the majority of oil and gas installations specify hot bending at elevated temperature. [10]-[12]

This project investigates the cold-roller-bending of plain carbon steel hollow sections for use in construction applications. The process of cold-roller-bending is widely considered to be an art rather 
than a science and therefore relatively little is known about the effects of the process on the physical and mechanical properties of the steel. In many cases it is assumed to be negligible[2] and therefore ignored by the standards and many of the guidance notes. Thus, the aim of this exercise was to determine and compare the effects of the cold bending process on the properties of hot-finished and cold-formed, plain-carbon steel, high-frequency induction (HFI) welded tubes.

There is no current EU, British or American standard that specifies changes in the mechanical properties brought about by the bending process or a maximum allowed curvature. This is because the properties are considered to largely remain the same as the original unbent material and that any alterations in these properties are made up for by the shape of the section.[3]-[5] This project refutes this assumption. Hot-finished and cold formed hollow sections were curved using rollerbending equipment to a range of strains with increasing curvature.

The project has examined all of the requirements outlined by a structural engineering calculation, including the tensile strength, impact resistance, hardness and geometric resistances of the cross section. This has been performed in the hope of determining the most suitable section for the coldbending process.

The testing has determined that the process of cold-roller bending can be detrimental to many of the properties detailed above. All of the mechanical, mechanical and geometric properties examined in the experimentation were affected in some way. For example, the cross-sectional geometry of the section can be significantly altered by the bending process as the stresses involved distort the cross section of the tube. This leads to differences in the geometric properties when compared to the original unbent section. These properties include the sections resistance to bending and torsion. The tensile properties of the sections are also affected. During testing it was determined that cold-roller bending of steel leads to significantly reduced yield strength where the material is in compression during bending. This reduction in the elastic capacity of the section has been determined to have been caused by the Bauschinger effect; a phenomenon that occurs in many metals that leads to a reduction in capacity to resist stress and therefore yield stress of a section is reduced. The effect is observed when the section has underdone pre-strain beyond the materials yield point in the opposite direction to the current deformation. Further investigation around the Bauschinger effect revealed that it occurs when the section is first elongated and then compressed as well as where the section is compressed before elongation. For a number of applications, in Civil design, the plastic properties are not normally considered as a structure is not commonly designed to operate within its plastic region when in service. However, sections that have been curved using cold-roller bending will yield at significantly reduced stresses and therefore 
the plastic properties should be considered, particularly if the section is being used in a position where the bending loads are being reversed (from that of bending), for example, as an archway. Furthermore, this reduced capacity to resist bending will lead to spreading of the archway when loaded from above. This will lead to increased lateral load being exerted on a support structure which must be considered when designing a structure using curved steelwork.

It was observed that both the hot-finished and cold-formed sections experienced a similar reduction in the yield strength during tensile testing (at the intrados) however; hot-finished sections regained their properties with further cold deformation more rapidly than the equivalent cold-formed product. This observation, coupled with improved impact resistances and superior geometric properties lead to the conclusion that hot-finished sections are considered to be more suitable for the cold-roller bending process. 


\section{Background Information}

\subsection{Section Types}

A number of different hollow section types were examined during the project with a focus on HFI welded hollow sections. TATA Steel tubes UK produce HFI welded products in many shapes and sizes, including circular, elliptical, square and rectangular sections. In recent years the product range has been simplified to ease the process of choosing the correct product for the final application. Structural hollow sections produced by TATA Steel are branded either as Celsius (hot finished) or Hybox (cold formed). TATA steel's production process for HFI welded structural hollow sections is described below:

\section{Structural High Frequency-Induction Welded (HFW) tubes \& Box sections (Hot- finished)}

Hot rolled strip, produced in TATA Steel Port Talbot is sent to the tube mills at Corby or Hartlepool by rail in the form of coils. At Corby, the wide coils are slit into narrower strips for tube production. Tube forming is a continuous process so the strips are first welded together, end-to-end. The process of welding the strips together cannot currently be carried out while the strip is in motion. So, as the tube mill runs continuously, an accumulator is used to gather strip and thereby allow sufficient time for the welding process to take place without having to stop or slow the tube production process. During tube production the strip is cold formed into a round tube shape using rollers. This can be seen in Figure 1. The HFW welding process involves heating of the strip edges using a high frequency alternating current passing through an induced by an induction coil. The coil produces a strong magnetic field that rapidly alters its orientation with the alternating current, this causes the strip edges to melt which are then forcibly pressed together and cooled, fusing them together to form a weld. During welding, a bead is formed on both the inside and outside surfaces. This is formed as the molten metal is squeezed out when the weld is being formed under high pressure. This bead is always trimmed off the external surface but for pressure or conveyance applications it is also removed from the bore of the tube. In these cases it is chipped into small sections before being removed with a scarfing tool. The tube weld is then assessed for defects using eddy current testing. This test will detect and mark the sections where the coil ends were bonded together, along with other forming defects. This allows automatic equipment to cut out the defective material for inspection. The tubes are then cut to fixed lengths for further processing, the actual lengths depending on the mill concerned and subsequent processing involved. For pressure tubes the remnants of the internal bead are removed from the bore using an air-blast. In the case of the hot-finished hollow sections, two further forming and heat treatment steps are performed. 
These extra steps are called normalising and reshaping. The normalising process is performed at temperatures exceeding $900^{\circ} \mathrm{C}$ and is implemented to homogenise the microstructure, removing the heat affected zone (HAZ) around the weld-line by re-crystallising the steel and eliminating any internal residual stresses from the section. This microstructure can be seen in Figure 2 and Figure 3. Any oxide scale generated in the furnace is then removed using high pressure water jets before reshaping. The reshaping process is used either to alter the diameter of the section or its shape from a round parent hollow to an elliptical, square or rectangular profile. The number, position and shape of the rollers in the mill-stands control the final length, diameter and shape of the final section. After cutting to length, the sections are allowed to cool in still air on cooling racks. The tubes are rolled regularly on the racks to prevent differential cooling which would results in deformation of the section causing a curved final shape. After cooling the sections are packed in bundles ready for dispatch.

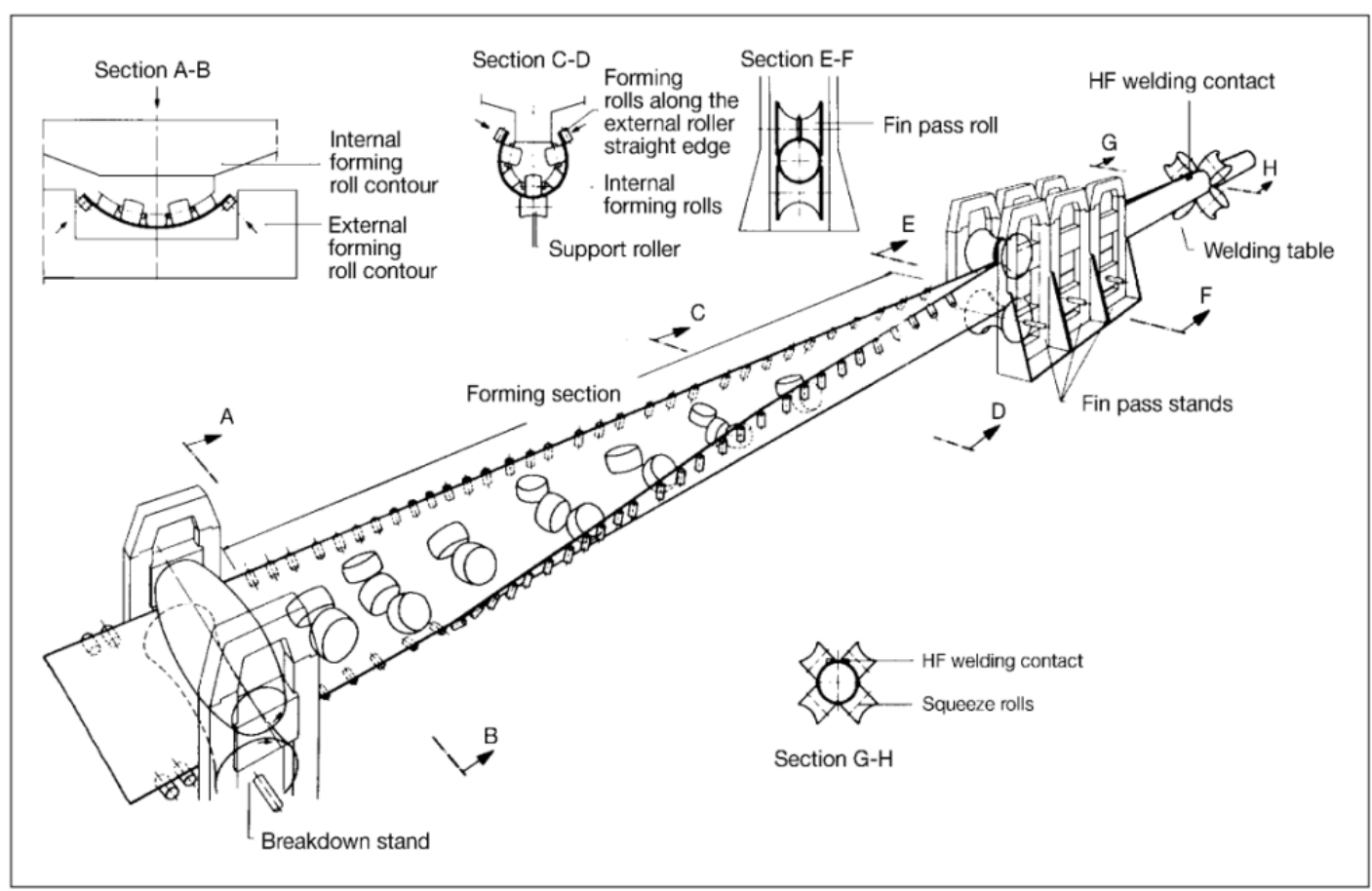

Figure 1-Tube forming process (welded)[1] 


\section{Microstructure}

The welded tube structure is a Hypoeutectic and is primarily fine ferrite grains with areas of Pearlite throughout. It has a fine microstructure due to recrystallisation during normalising.

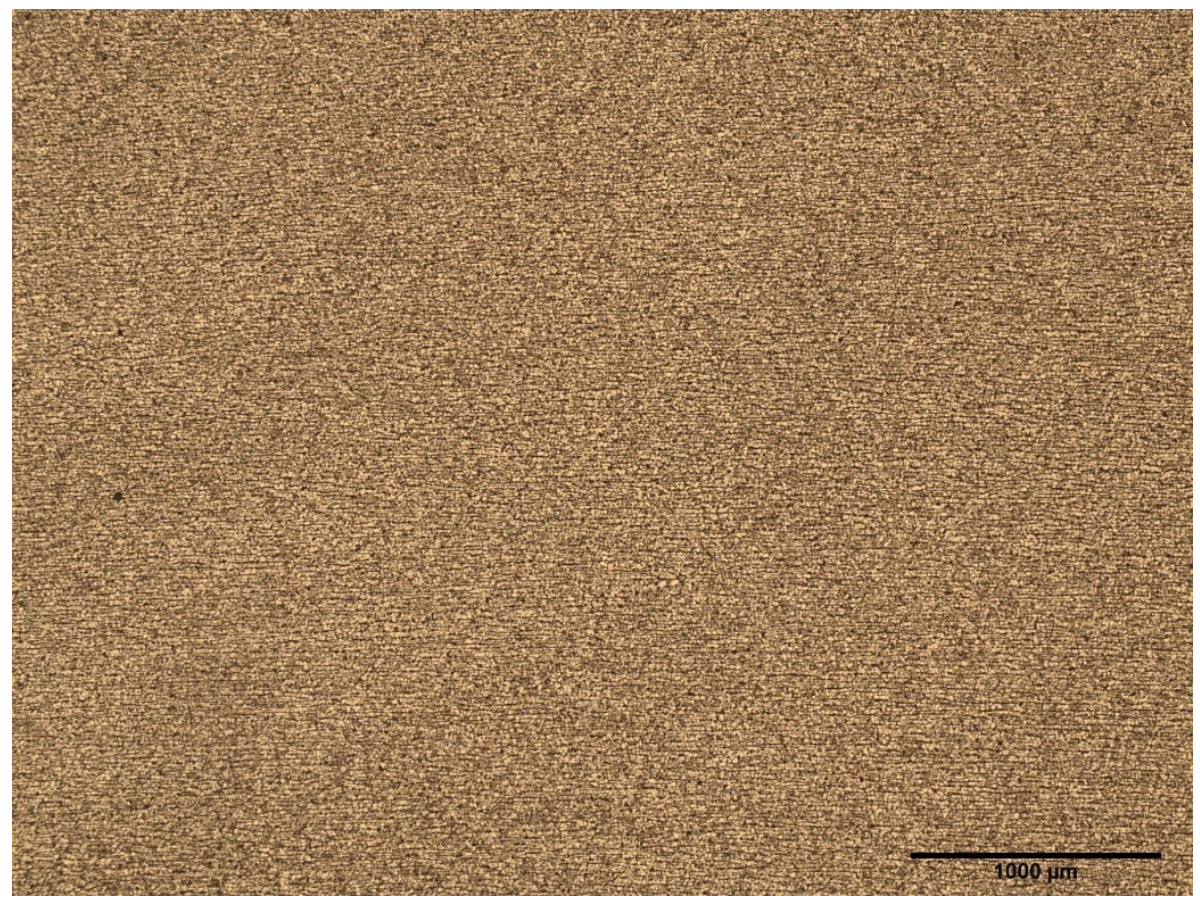

Figure 2-Body micrograph of a fully normalised CHS[2] 
The microstructure around the weld is similar to the body; however, the longitudinal banding of the microstructure is diverted towards the internal and external surface adjacent to the weld as the molten material is ejected during the forming process. This shape is not removed during the normalising process and can be seen in figure 3 .

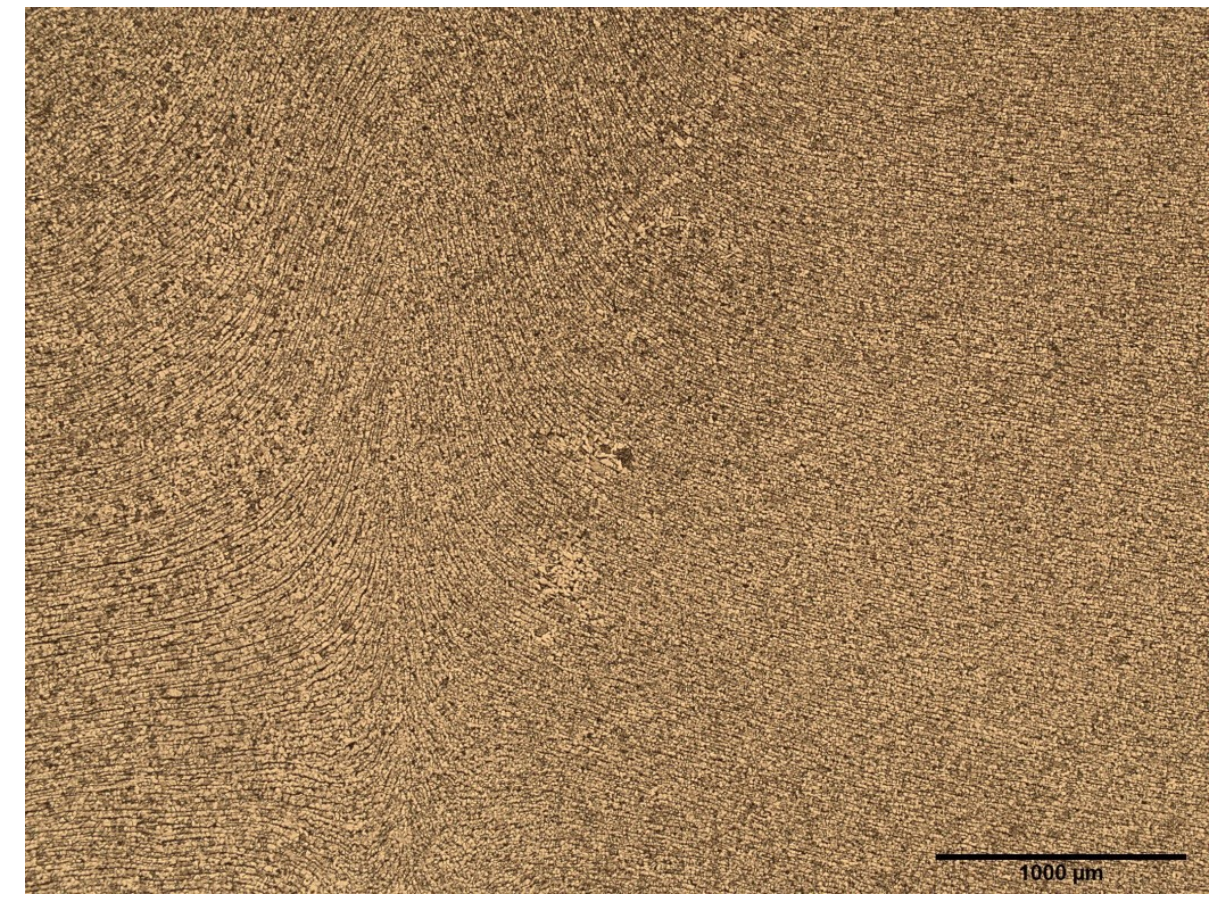

Figure 3-Weld-line micrograph of a fully normalised CHS[16]

\section{HFW Tubes \& Box Sections (Cold Formed)}

Cold formed tubes are produced initially in the same way as the hot finished tubes but don't undergo the subsequent normalising or hot reshaping process. Instead the shaping process to a square or rectangular section is carried out at ambient temperature. As a result, this leads to considerable stress being introduced in the corners of the section. The corner radii of cold formed tubes cannot be as small as the hot finished equivalents of the same dimensions.

The microstructure of the cold formed products is also very different from the hot finished equivalent. Cold work of the material has led to elongation of the grains. This elongation of the grain structure affects the mechanical properties of the product and leaving residual stresses which work to expand the tube. These residual stresses are often measured by performing a split ring test, where a short length of tube is slip and the expansion of the section is measured. Rapid recrystalisation has taken place at the weld line which leads to a hard and fairly brittle structure. The heat affected zone (HAZ) and weld are clearly visible when a cross section is prepared and examined under a microscope. 


\subsection{Section Bending Processes}

There are two main section bending methods, hot induction bending and cold roller bending. Cold roller bending is generally employed for the curving of structural steels due to the lower costs associated with the method.

The different curving mechanisms have advantages and disadvantages. This particular project investigates cold-roller bending and its effect on the mechanical and geometric properties of the steel sections processed in this way.

\section{Cold Bending}

Cold-roller bending is a relatively simple curving procedure that involves progressive bending of a section during a number of passes through a set of bending rolls. The shape of the rolls can be altered to suit the shape of the section to be bent. Each pass through the rolls reduced the radius and the process is repeated until the required degree of curvature is achieved. A schematic diagram of the bending process can be seen below.

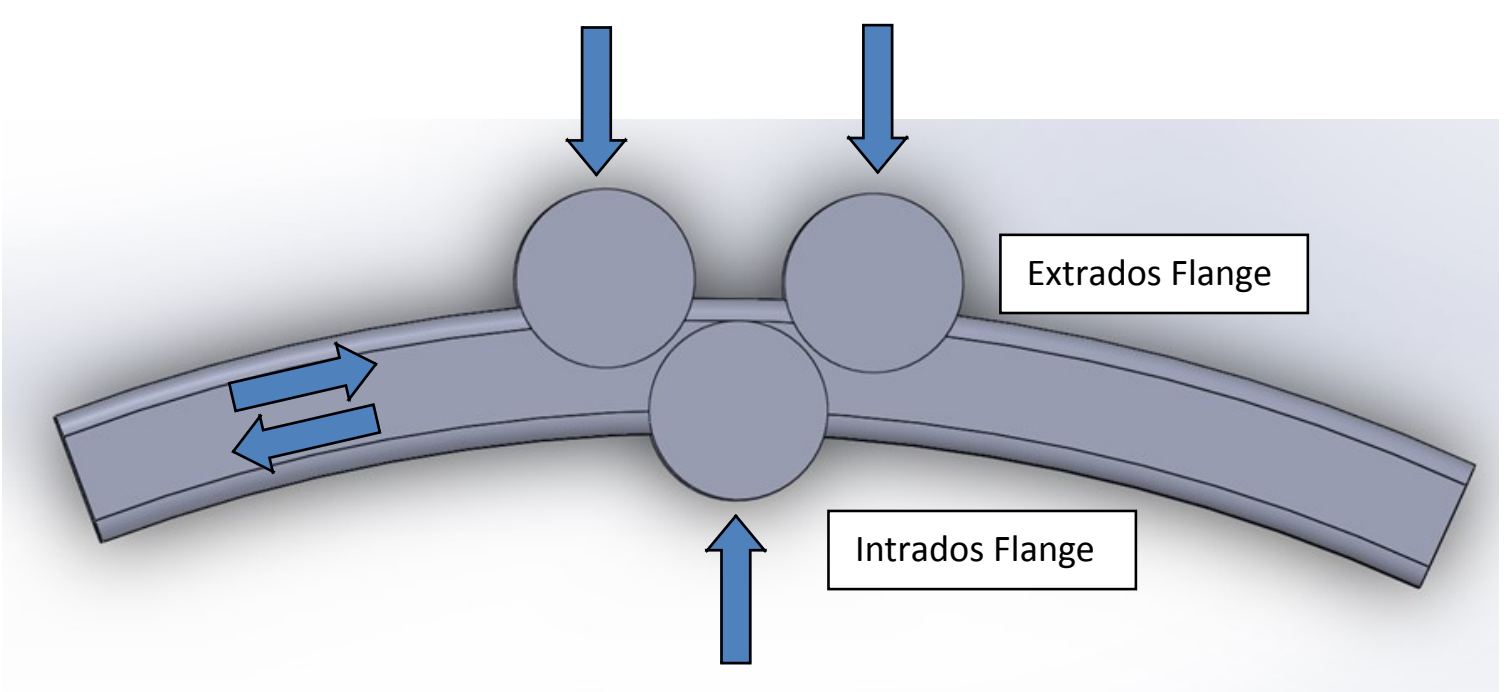

Figure 4-Forming roller force application points during bending

This method of bending relies on the plastic deformation of the material so that the section retains its shape after the load has been removed[1], [13]. Cold bending can be problematic when thin walled sections or tight radii are to be produced. The pressure exerted by the rollers is high and therefore can lead to wall collapse or distortion in tubes if an internal mandrel is not also used.

The minimum radius to which a section can be curved without significant distortion depends on the section properties and the bending methods used. Advances in technology and curving techniques mean that minimum radii continue to be reduced. During the cold roller-bending process, the 
material is strained beyond the elastic limit, and a permanent set is created in the material. In the case of a beam, the outside flange is stretched and the inner flange is compressed. There is no UK, European or American Standard that predefines or limits the radius to which structural sections can be cold curved for use in construction. This is because for standard low-carbon steels, the strain undergone during the bending process is not usually considered detrimental. The material is

(a) Snap-through

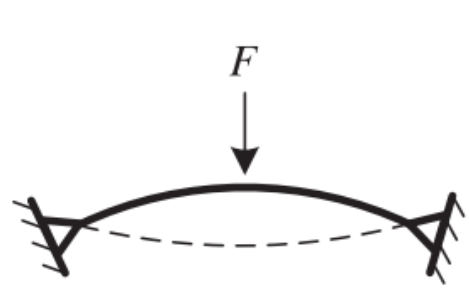

(b) In-plane buckling

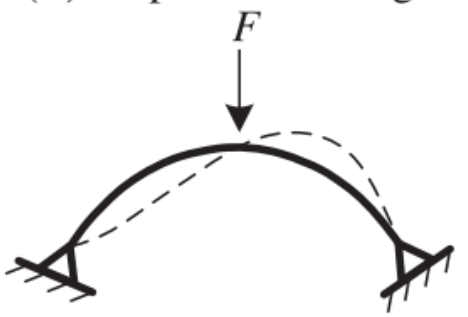

(c) Out-of-plane buckling

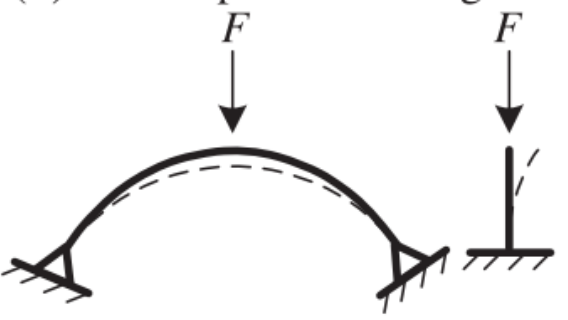

Figure 5-Global instability phenomena for arches that transfer loading to their abutments: (a) Snap through, (b) In-plane buckling, (c) Out-of-plane buckling[2]

thought to exhibit the same elastic characteristics in the same elastic range. However, it is generally accepted that the strain capacity is reduced. [2], [14]-[19] It is known and accepted in industry that the steel sections become strain hardened when using the cold roller-bending process. The amount of strain hardening depends on the curvature achieved and the geometry of the section. The Bauschinger effect, which is examined in detail in a later chapter is not considered in engineering calculations. A tensile test on a sample of steel that has been cold roller-bent will show a small loss in ductility and a higher ultimate tensile strength when bending in the same direction is resumed.[2], [20] Even though there is a loss of ductility, for normal structural applications the effect is considered minimal and for most applications can be ignored. Despite there being no standard for engineers to conform to, the Steel Construction Institute has produced a guidance document to aid in the design of curved steel structures [2]. This section investigates the document and covers a number of aspects of steel design including residual stresses, out of plane bending and bucking of the section in situ. Some of the in-situ buckling modes are shown in Figure 5. It also includes a number of equations in order to take the altered section geometry into account when performing calculations.

Cold curving is known to alter the residual stresses in members[2], [19], [21]. The magnitude of these residual stresses remaining after the curving process is thought to depend heavily on the section properties in the direction of curvature. It is thought that the residual stresses do not affect the cross-sectional resistance of a section. As a section passes through the bending rolls, the strain exceeds the yield strain, causing the required plastic deformation. As it leaves the rolls elastic spring back occurs, restoring equilibrium in the cross section. 
Figure 6 shows the stress distribution assuming that the extreme fibre strain reaches $K \varepsilon_{y}$, where: $\varepsilon_{y}$, is the yield strain of the steel. The figure also demonstrates that not all of the section reaches yield stress. In which case a plastic hinge would occur and the section would fail. Close to the neutral axis, stresses remain in the elastic region. It is generally considered that the strain in the sample is a linear distribution from maximum extension at the extrados to maximum compression at the intrados of curvature. Thus it is assumed that the strain at the neutral axis is zero.

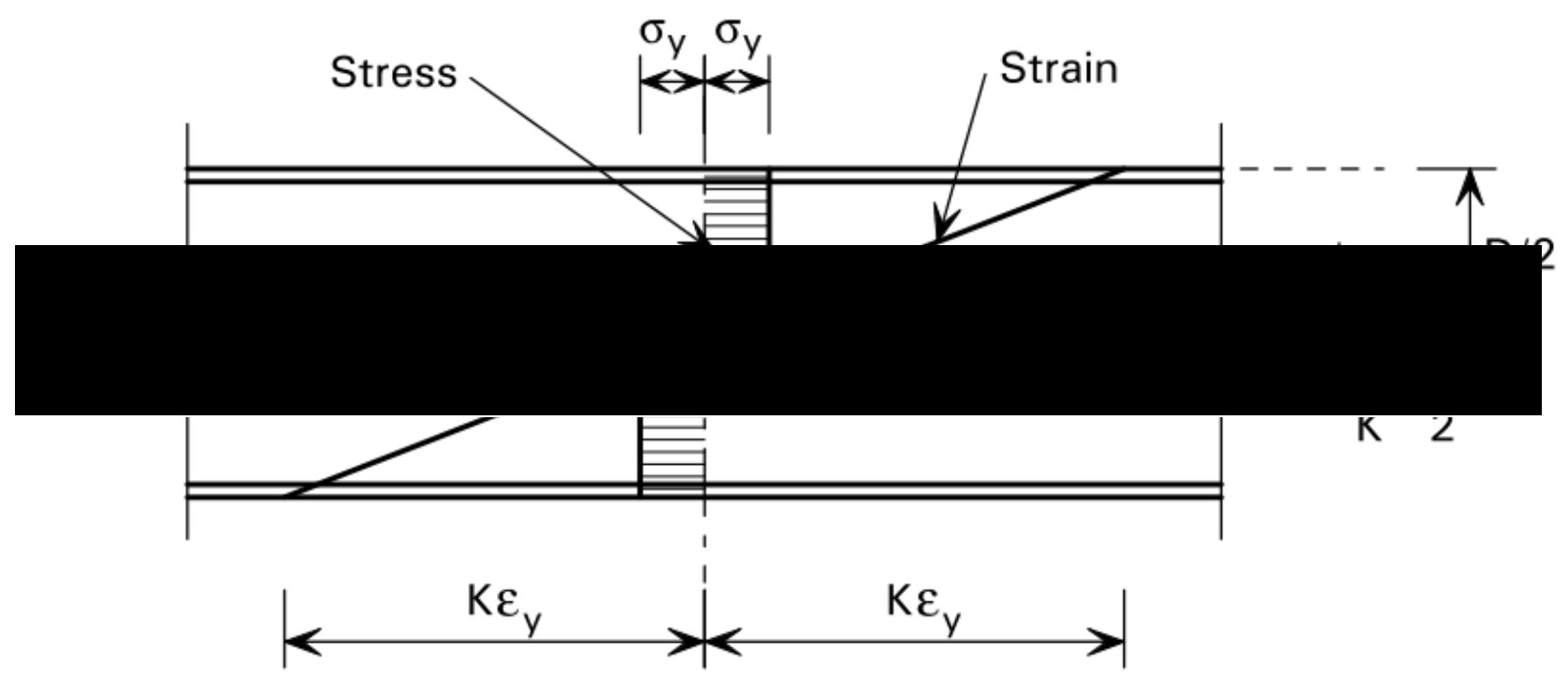

Figure 6-Stress and strain in a section while in the bending rolls[2] 


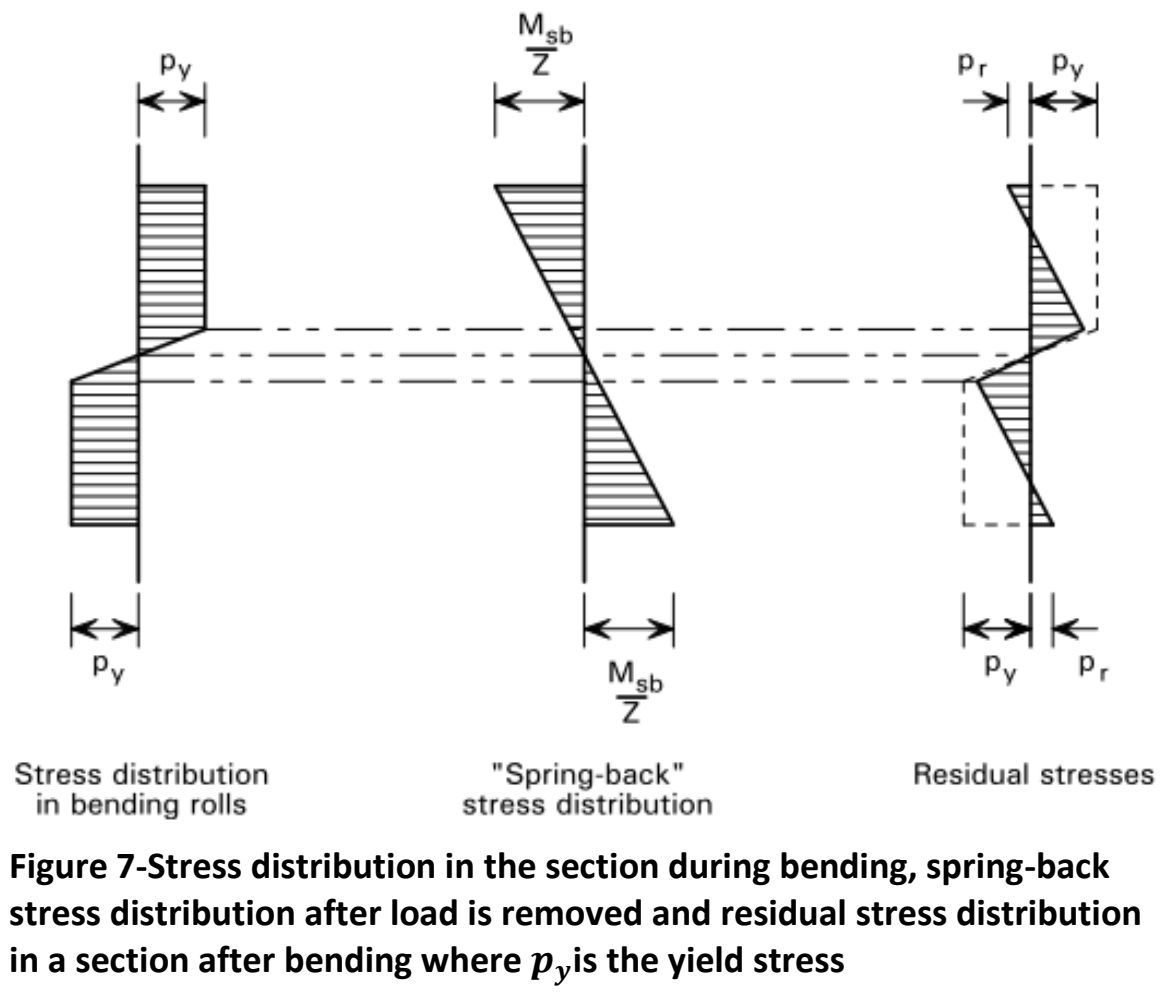

Figure 7 shows the stress distribution in the bending rolls which is induced by the bending moment caused by the rolls, $M_{b r}$, and the elastic "spring-back" moment, $M_{s b}$, which restores equilibrium such that[2], [21]:

$$
M_{b r}+M_{S b}=0
$$

\section{Equation 1-Residual Stress Equilibrium[2]}

The literature suggests that equilibrium is restored after the bending load is removed and the material springs back. The "spring-back" moment is elastic, therefore, the spring-back strains at the extreme fibres, exceed yield strain, producing the stress distribution shown in Figure 7.

The residual stresses at the extreme fibres, $p_{r}$, are therefore of opposite sense to the stresses induced by the bending rolls and are given by:

$$
p_{r}=\left(\frac{M_{s b}}{\text { Plastic Section Modulus }}\right)-p_{y}
$$

\section{Equation 2-Residual stress calculation[2]}

This residual stress opposite to the direction of bending is likely to reduce the force required to restraighten the section. As plastic deformation increases, the strain in the extreme fibres increases, however; elastic spring-back is unchanged and is always equal to the elastic strain. 
To estimate the residual stresses, the bending moment caused by the bending rolls are usually assumed to be the plastic moment

$$
M_{p} \approx p_{y} S
$$

Where: $\mathrm{S}$ is the elastic section modulus

\section{Equation 3-Plastic Moment}

The spring-back stresses, $p_{s b}$, are therefore given by :

$$
p_{s b}=\frac{p_{y} S}{Z}
$$

\section{Equation 4-Spring-back Stress}

And the residual stresses at the extreme fibres are:

$$
p_{r}=p_{s b}-p_{y}=\frac{p_{y} S}{Z}-p_{y}
$$

\section{Equation 5-Residual Stress[2]}

For square and circular hollow sections, the ratio of $S / Z$ is the same in either direction and is usually quite small. However for universal beams the ratio varies depending on the bending plane. Therefore the residual stress is usually less when compared to universal sections, approximately $\approx$ $0.2 p_{y}$ [2] when compared to $0.5 p_{y}$ for open sections curved on plan. Therefore, if serviceability limit state stresses are imposed, (typically approximately $0.6 p_{y}$ ) the additional residual stress could lead to plasticity at working load, particularly in open sections such as universal beams. However, the guidance notes state that in most cases, deflection is likely to be the governing criteria and the serviceability limit state is not likely to exceed yield. This is contrary to evidence of the Bauschinger effect. 
During bending it is expected that there will be out of plane bending of the flanges. The flanges of curved members subject to in-plane bending or axial loads must also resist the out-of-plane component of loads resulting from the curvature of the member. This applies to both open sections and hollow sections.

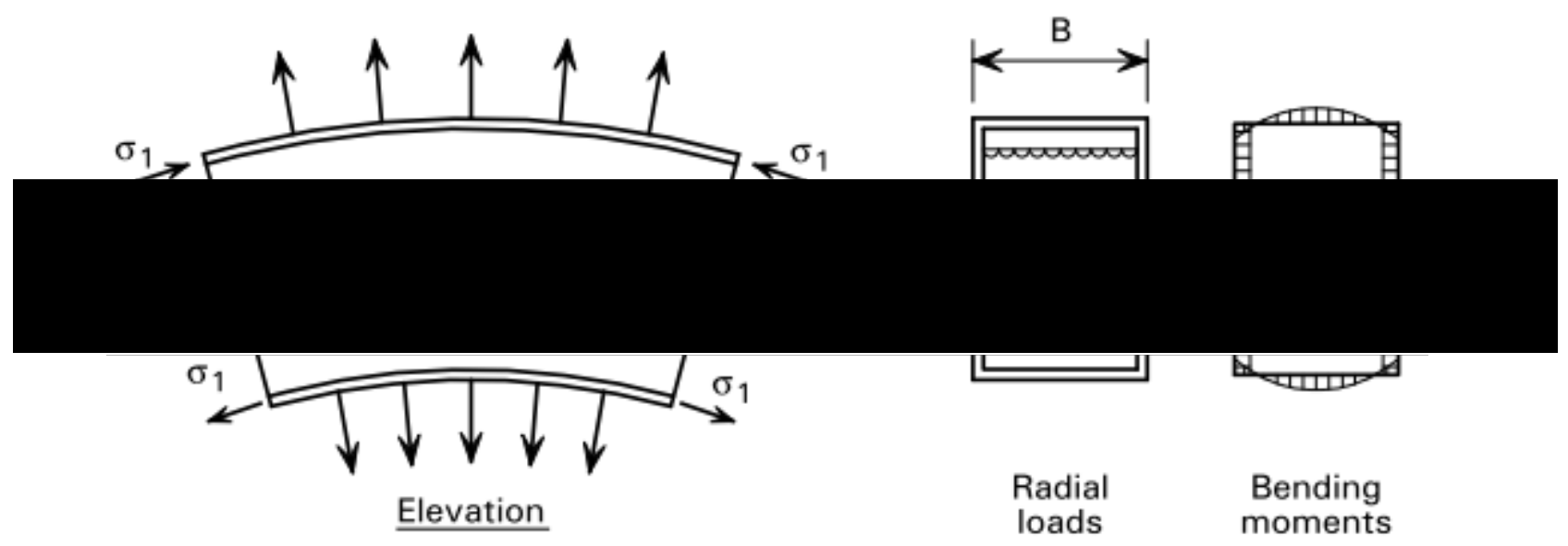

Figure 8-Out of plane bending of square hollow section walls

Figure 8 shows a cut-through of a typical RHS subject to bending. Due to the direct stresses $\sigma_{1}$, the forces at the ends of these slices are not in equilibrium because they are inclined. This inclination causes a radial component of load all along the slice, which must be resisted by out-of-plane bending in the flange.

For the intrados and extrados flanges of box sections, the maximum allowable bending moment during curving cannot exceed:

$$
M=\left(\frac{T \sigma_{1}}{R}\right)\left(\frac{B^{2}}{8}\right)
$$

\section{Equation 6-Maximum allowable moment to prevent failure due to out of plane stresses[2]}

Where $\mathrm{R}$ is the radius of curvature and $\mathrm{T}$ is the thickness of the flange.

The out of plane bending stress is given by:

$$
\sigma_{2}=\frac{3 \sigma_{1} B^{2}}{4 R T}
$$

Equation 7-Out of plane bending stresses generated by axial deformation stresses[2] 


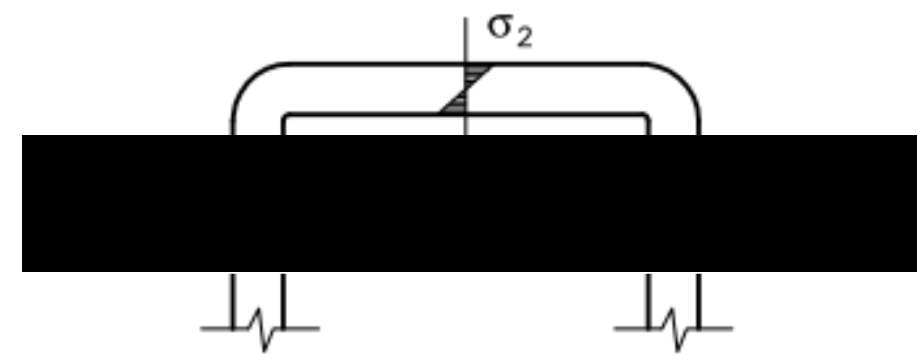

Figure 9-Out of plane stresses in the flanges[2]

When calculating the reduced section capacity due to these out of plane stresses, the calculations are based on the elastic modulus of the flange rather than the plastic modulus. This conservative approach prevents buckling of the flanges. [2] The out-of-plane loads reduce the cross-sectional resistance of a member because of the transverse bending stresses, $\sigma_{2}$ which are accounted for using the von Mises relationship.[2], [22] Out of plane bending stresses should always be taken into account when calculating any resistance of a hollow section. Some out-of-plane stresses are locked in during the cold rolling process. These are small enough to be ignored.[2] 


\section{Induction Bending}

The process of induction bending does not rely on rollers like cold bending and the section is only passed through the equipment once.[5] The process is slower than cold bending with a bend speed close to $50 \mathrm{~mm} / \mathrm{min}$ and involves using a hydraulic ram to push the section through a specially shaped induction coil. Induction heating, when applied to hot bending of tubes, consists of a single induction coil to heat a relatively narrow band of the tube. The coil generates an intense localized magnetic flux and "induces" an electric current to circulate within the tube wall directly below the coil.[6-9] However, there is no residual magnetism. It is the induced circulating current and the material resistivity which effectively generates the heat necessary for hot bending. The induction coil can be engineered to give different heating affects such as a wide heat band to account for thermal conduction into thick tube walls, as well as various configurations of cooling water spray.[6]

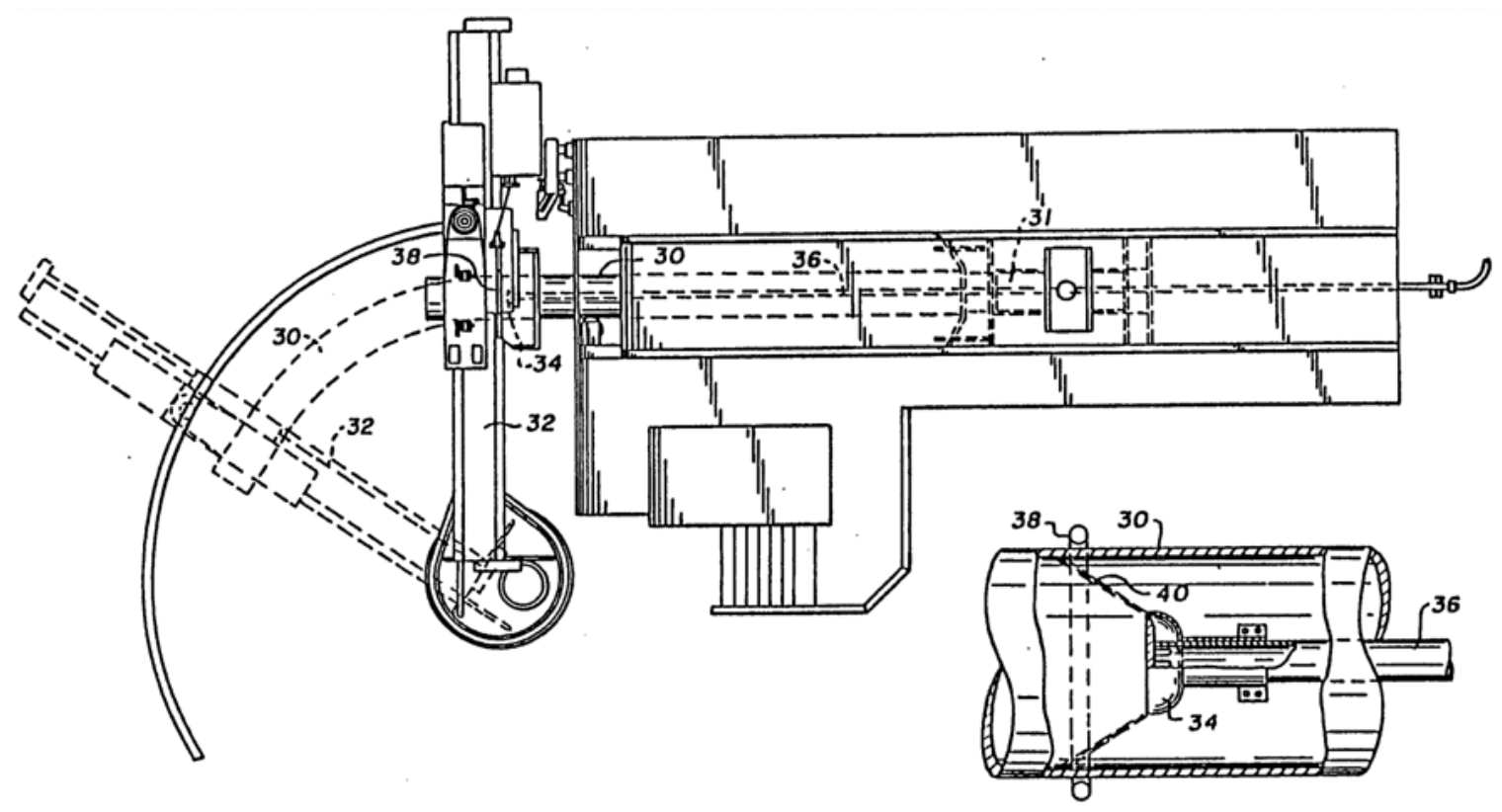

Figure 10-Schematic Diagram of an Induction Bending Machine[16]

The coil heats up the section, increasing the malleability for bending. It is therefore inevitable that hot bending will affect the properties and dimensions of the tube. The challenge is to design and control the process such that acceptable material properties are achieved. The induction heating process has the unique capability to generate a narrow circumferential heat band. The tube bending process exploits the generated heat band as a "plastic hinge".[12] The use of hot bending allows sections to be bent with relatively little force due to the significantly lowered yield strength of the austenitic phase created in the section as it passes through the coil.[23], [24] An additional advantage is that all the plastic strain due to bending is confined to the narrow heat band and this minimises the tendency for tubes to become out of round and lowers the threshold for wrinkling. 
[12], [25], [26] Usually, the heated section is immediately cooled by water sprays after bending; this also helps to ensure better dimensional control when compared to heating alone.

The water spray is often set up in such a way as to maximise the cooling rate.[12] The bending is done using a rotating arm of fixed length which is attached to the front of the section. As the section is pushed through the coil, the arm rotates about an axis a set distance, away from the section to be bent. The length of the arm determines the radius of the bend. All bending takes place within the heated zone. It is plausible to assume that this region where bending can occur may increase when the section in unquenched, the reason for this is that a larger section of the member/tube remains at a highly elevated temperature. This also leads to further grain growth of the microstructure, leading to a softer microstructure. As stated above, hot bending allows thicker walled sections to be bent with considerably reduced applied forces and tighter curvature.[10] The high forces required during cold bending of thick walled sections can lead to buckling and ultimately collapse of tube walls, the reduced force required for induction bending minimises this risk.

The reduced compressive strength of the steel at the heated zone could lead to buckling of the compressed inner surface leading to a non-uniform, undulating surface finish.[12] The extrados is likely to undergo wall thinning due to tension caused by the relative increase in length. The phenomenon that causes this change in wall thickness during bending is known as Poison's ratio. It has been found that induction bending can lead to a wall thinning of around $15 \%$ at arc lengths of around 5R.[12] Expected distortions for general bending can be estimated from tables. Actual distortions may vary from predicted values due to the particular bending process requirements such as speed, temperature, cooling method, coil design, material type and chemistry.[27], [28]

There are three key process parameters for induction bending which affect the material properties: bend speed, peak temperature and cooling rate. The start and stop procedures are secondary process parameters, which also affect the properties of the section.[12] The effect differs between machines (and often even between operators) and depends on the sophistication of the control process for that equipment. Modern HFI welded line pipes are low carbon micro-alloyed steels. Induction bending can be performed at temperatures that range from just above the critical temperature for steel to over $1000^{\circ} \mathrm{C}$ but are typically carried out at temperatures between 875 and $1075^{\circ} \mathrm{C}$ depending on the chemistry, [28] which is beyond the austenitising temperature where recrystallisation takes place. Over this temperature range the dissolution of micro-alloyed elements increases with temperature. For a given chemistry, the peak temperature achieved during induction heating and the rate of cooling determine the final material properties. There is a clear link between induction bending temperature, ultimate tensile strength (UTS) and hardness.[12] The strengthening 
mechanism is a combination of grain size effects, the solution and re-precipitation of micro-alloying constituents and the formation of low temperature transformation products such as Martensite and Bainite. To consistently achieve high strength and toughness directly from the induction bending equipment, the peak temperature and cooling rate needs to be carefully controlled and this best practice must be determined and supported by physical testing. For a fixed speed and constant cooling rate (used in most companies employing the induction bending method), the peak temperature is controlled by the level of induction power applied during the bending process. The cooling rate is determined by both the speed of bending and cooling water spray system comprising pressure, volume and apertures (type and number).

An important consideration for induction bends is the use of post bend-heat treatments including annealing, normalising, tempering, together with quench and temper.[29] In some cases there may be a conflict between the process parameters required to achieve the required material properties. In thick walled, high strength tube, the process parameters needed to achieve the yield and tensile strength requirements may cause the outside surface hardness restrictions to be exceeded.[15, 16] The only way to solve this problem is likely to be the application of a post-bend heat treatment but this is restricted by the size and availability of suitable furnaces. There are very few furnaces available which are capable of heat treating induction bends made from large diameter pipe. This is especially problematic for bends which require quench and temper heat treatments and annealing. Incorrect use of post-bend temper heat treatments may introduce more problems: in particular, a tempering heat treatment required for the bend area may adversely affect the unbent straight section on each end of the bend. Annealing can also be a problem as it can result in sagging of the section in the furnace. Due to a combination of size range of HFI welded pipe and chemistry; it is generally well suited to the induction bending process. Heat treatment is therefore rarely required for induction bends formed from $\mathrm{HFI}$ welded tubes unless the cooling rate is not controlled adequately.[12], [16], [28]-[31]

It is thought that induction bending at around $900^{\circ} \mathrm{C}$ can lead to micro-cracking attributed to a ductility trough that can exist at this temperature.[32] Babock-Oberhausen, and other commercial companies now bend below this trough at a temperature around $750^{\circ} \mathrm{C}$ to avoid micro-cracking and to reduce power costs. Inherently, the cost of induction bending is greater than that of cold bending, as considerable heating is involved, requiring high currents.

Induction heating is the process of heating an electrically conducting object by electromagnetic induction, where eddy currents are generated within the metal, resistance leads to heating of the section. An induction coil consists of an electromagnet, through which an alternating current is 
passed. Heat can be generated in materials that have significant relative permeability with magnetic hysteresis losses. The frequency of $A C$ used depends on the object size, material type and the penetration depth. The larger the section that is being heated the lower the frequency needed. Thick metals require a frequency of $\mathrm{AC}$ between 5 and $30 \mathrm{kHz}$ whereas; smaller work-pieces or shallow heat penetration requires a frequency of between 100 and $400 \mathrm{kHz}$.[30], [31] Induction heading is most effective for Iron and its alloys, which are heated rapidly due to their ferromagnetic nature.[33]

Magnetic materials improve the induction heat process because of hysteresis. Materials with high permeability, for example, between 100 and $500 \mathrm{H} / \mathrm{m}$, are easier to heat by induction. Hysteresis heating occurs below the Curie temperature, where materials lose their magnetic properties. High permeability below the Curie temperature in the work-piece is useful. Temperature difference, mass, and specific heat influence the work-piece heating.[30]

The energy transfer of induction heating is affected by the distance between the coil and the workpiece. The closer the work-piece is to the coil, the greater the heat transfer.[30] Energy losses occur through heat conduction from the work-piece due to convection, and thermal radiation. However the air jets used help to prevent thermal conduction away from the heated zone behind the induction coil. The coil is usually made of copper tubing. The diameter, shape, and number of turns influence the efficiency and field pattern[12].

To understand where the risks lie for curving of steel sections, it is important to understand the characteristics of the various types of tube that are available and how they relate to the process.

\section{HFI Welded Tube}

Most tube induction bends are performed on HFI welded tube with a range of wall thicknesses and grades so that the necessary material properties can be produced directly from the induction bending equipment without any further heat treatments.

Steel tubes manufactured in modern HFI welded tube mills are often produced from thermomechanically controlled, rolled steel strip with chemistries to meet grade and high speed seam weldability requirements. The properties imparted by this specialist cooling method are lost during heating of the section for induction bending, however, careful control of the temperature and cooling rate when curving these sections can produce a product similar to that of the unbent material. [12], [28], [34] 


\section{Seamless}

The manufacturing process for high strength seamless carbon steel tube is quite different to that used to make tube from rolled plate or strip, as mentioned earlier. Seamless tube is hot-finished to achieve the required diameter and wall thickness; it is then heat treated to achieve the required strength and toughness. Seamless tube mills naturally design chemistries to suit the rapid internal and external mill quench and heat treatment process. Induction bending in its current state is limited to external water spray cooling at relatively slow speeds and therefore cannot achieve the same quenching rate as large scale tube mills. This means that, for certain sections, it may be necessary to perform a full body post-bend quench and temper heat treatment; otherwise only downgraded material properties may be achieved off the bending process.[12] 


\subsection{Common Phases of Steel}

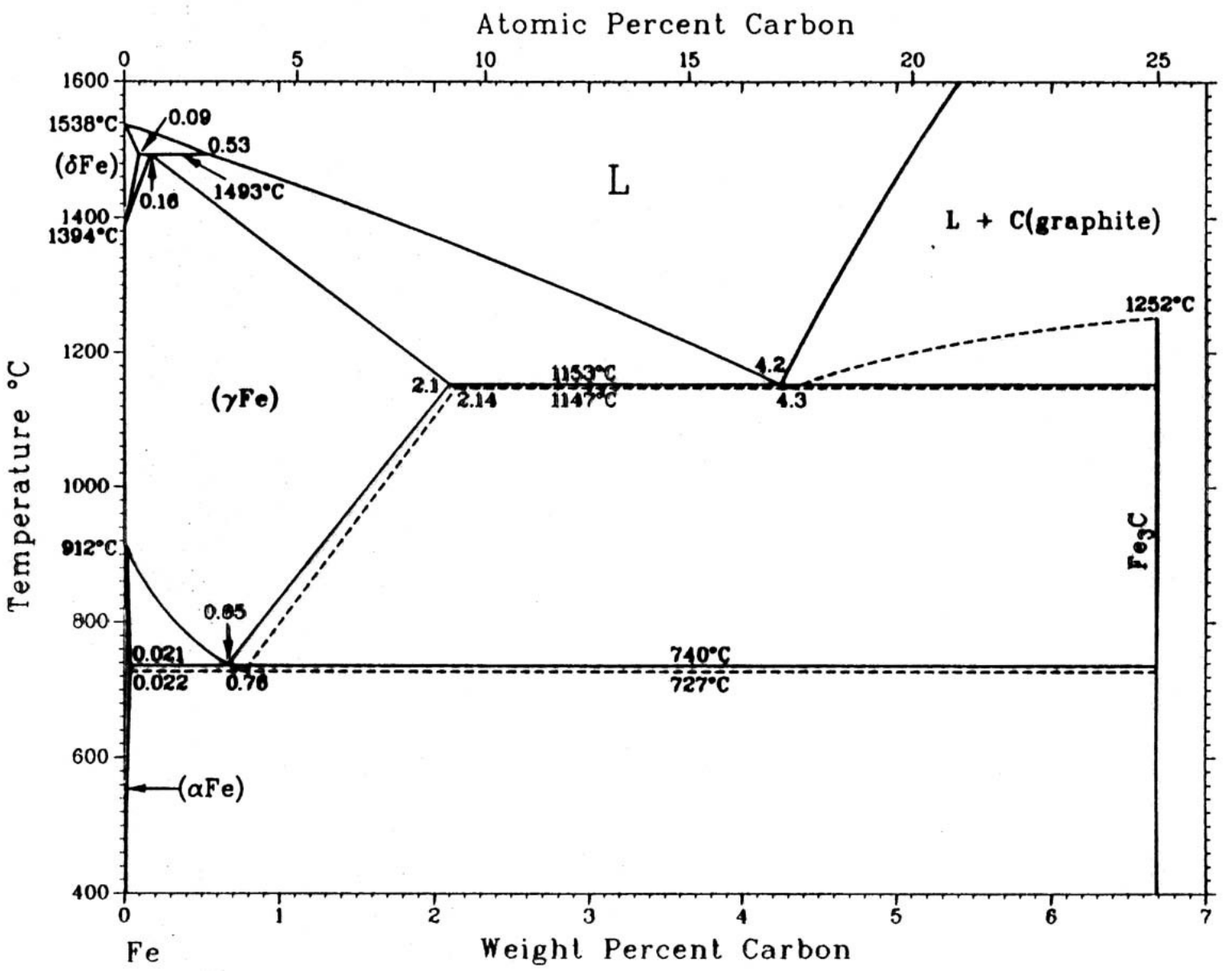

Figure 11-Iron-Carbon Phase Diagram

Figure 11 shows the iron-carbon phase diagram. Steel in its most basic form is an alloy of iron and carbon, this is known as a plain carbon steel. However, a number of other chemical additions can be used to affect the desired properties of the final steel. All phases depicted in the diagram are produced from slow heating or cooling rates allowing time for diffusion based reactions. Metastable states, such as Martensite and Bainite can be produced through rapid heating or cooling rates[24]. The steel portion of the phase diagram shown covers the range between 0 and $2.08 \mathrm{wt} \% \mathrm{C}$. The cast iron portion of the diagram covers the remaining range between 2.08 and $6.67 \mathrm{wt} \% \mathrm{C}$. The steel portion of the metastable diagram can be divided further into three subdivisions, hypoeutectic, eutectic and hypereutectic steels. All of the samples used in this project are hypoeutectic.

Steel that contains less than $0.68 w t \% C$ is classed as hypoeutectic. Steel containing $0.68 w t \% C$ is classed as eutectic. Above $0.68 \mathrm{wt} \% \mathrm{C}$, they are classed as hypereutectic. The grades of steel used in this project contain between 0.14 and $0.17 \mathrm{wt} \% \mathrm{C}$ and are therefore hypoeutectic. The addition of other alloying additions can alter the eutectoid composition. Elements that stabilise austenite, 
decrease eutectoid temperature, whereas, ferrite stabilising elements increase the eutectic temperature. Some ferrite stabilising elements include: chromium, silicon, titanium and aluminium.

The hot-finished steel has been fully normalised during tube production, its composition will primarily be proeutectoid ferrite with a proportion of eutectic ferrite and cementite that forms the perlite structure[35]. Cementite is considered to be metastable and over a period of years can decompose to ferrite and graphite. The proportion of ferrite can be approximately determined using the following calculation:

$$
W t \% \text { Ferrite }=\frac{6.67-0.14}{6.67-0.02} \times 100=\frac{6.53}{6.65} \times 100=98.2 \%
$$

\section{Equation 8-Wt\%ferrite[35]}

The following sections describe a number of common phases that steel can comprise.

\subsubsection{Ferrite}

Ferrite, also known as $\alpha$-ferrite ( $\alpha$-Fe) or alpha iron are terms for pure iron, with a body-centred cubic (BCC) crystal structure. It is this crystalline structure which gives steel and cast iron their magnetic properties, and is a good example of a ferromagnetic material. It has a ultimate tensile strength of approximately $280 \mathrm{MPa}$ and is fairly soft due to the low percentage weight of carbon.[24] Mild steel (up to $\approx 0.2 \mathrm{wt} \% \mathrm{C}$ ) consist typically of ferrite, with increasing amounts of Pearlite as the carbon content is increased. Since Bainite and Pearlite each have ferrite as a component, any ironcarbon alloy will contain some ferrite if allowed to reach equilibrium at room temperature. The exact amount of ferrite will depend on the cooling processes that the alloy undergoes as it cools from above its critical temperature. [24], [35]

In pure iron, ferrite is stable below $912^{\circ} \mathrm{C}$. Above this temperature, it converts to austenite, the face-centred cubic $(\mathrm{FCC})$ form of iron, also known as gamma-iron $(\nu-\mathrm{Fe})$. Above $1,390^{\circ} \mathrm{C}$, up to the melting point of $1,539^{\circ} \mathrm{C}$, the $\mathrm{FCC}$ austenite converts to a more stable $\mathrm{BCC}$ crystal structure called delta-ferrite $(\delta-\mathrm{Fe})$. Only a very small quantity of carbon can dissolve into the in ferrite lattice; the maximum solubility is $\approx 0.022 \mathrm{wt} \%$ at $727^{\circ} \mathrm{C}$ and $0.005 \%$ carbon at $0^{\circ} \mathrm{C}$. This is because carbon dissolves in iron interstitially.[24] Carbon atoms are approximately twice the diameter of the interstitial area, meaning each carbon atom is surrounded by a strong local strain field.

\subsubsection{Cementite}

Cementite; or iron carbide is a chemical compound of iron (Fe) and Carbon (C). The formula for cementite is $\mathrm{Fe}_{3} \mathrm{C}$. Cementite is made up of $6.67 \mathrm{wt} \% \mathrm{C}$; the remaining $93.3 \mathrm{wt} \%$ is $\mathrm{Fe}$. It has an 
orthorhombic crystal structure. Iron carbide is a hard, brittle material and is classified as a ceramic material in its pure form.[24], [35]

\subsubsection{Austenite}

Austenite; or gamma-iron ( $\gamma$-Fe) is the face centred cubic (FCC) form of steel and due to this structure has higher carbon solubility than the BCC alpha ferrite. The maximum solubility of carbon in austenite is $2.14 \mathrm{wt} \% \mathrm{C}$ which occurs at $1147^{\circ} \mathrm{C}$. Austenite is never stable in plain carbon steels at less than $727^{\circ} \mathrm{C}$, however; additional alloying elements can make it stable at room temperature. These elements are known as Austenisers, for example, Nickel, Molybdenum and Manganese ( $\mathrm{Ni}$, Mo, Mn respectively). Due to its FCC crystal structure, austenite is paramagnetic, meaning that is only very weakly attracted to a magnet. Austenite is easily work-hardened making it both strong and ductile.[24]

\subsubsection{Pearlite}

Pearlite is often said to be a two-phased, lamellar structure comprising of alternate layers of alphaferrite $(88 w t \% \alpha-\mathrm{Fe})$ and cementite $\left(12 \mathrm{wt} \% \mathrm{Fe}_{3} \mathrm{C}\right)$. It forms in some steels and cast irons. The lamellar appearance is misleading as the individual lamellae within a collection are connected in three dimensions; a single cluster is therefore an interpenetrating bicrystal of both ferrite and cementite. In a eutectic iron-carbon alloy, Pearlite forms via a eutectoid reaction as austenite slowly cools below the critical temperature of $727^{\circ} \mathrm{C}[23]$, [35].

The eutectoid composition of austenite is approximately $0.76 \mathrm{wt} \% \mathrm{C}$; steel with less carbon is known as hypo-eutectoid and will form a proportion of relatively pure pro-eutectoid ferrite crystallites. These ferrite crystals do not participate in the eutectoid reaction and cannot transform into Pearlite. Likewise steels with higher carbon contents are known as hyper-eutectoid and will form proeutectoid cementite before reaching the critical temperature. The proportion of ferrite or cementite forming above the eutectoid point can be calculated from the iron/iron-carbide phase diagram shown in Figure 11 using the lever rule.[24], [35]

\subsubsection{Martensite}

Martensite is formed in carbon steels by the rapid quenching of austenite. The cooling occurs so rapidly that carbon atoms are not able to diffuse out of the crystal structure (before the ferrite is formed) in large enough quantities to form cementite $\left(\mathrm{Fe}_{3} \mathrm{C}\right)$. As a result, the $\mathrm{FCC}$ austenite transforms to a highly strained body-centred tetragonal (BCT) form of ferrite that is supersaturated with carbon. The axial ratio of $c / a$ increases with increasing carbon concentration and decreases on 
tempering. The ratio decreases with increasing tempering temperature.[28] The shear deformations that result produce large numbers of dislocations, which is a primary strengthening mechanism of steels. The greatest hardness observed in Pearlitic steel is $\approx 423 \mathrm{HV}$ whereas Martensite can be greater than $\approx 772 \mathrm{HV}$.

The martensitic reaction begins during cooling when the austenite reaches the Martensite start temperature $\left(M_{s}\right)$ and the parent austenite becomes mechanically unstable. As the sample is quenched, an increasingly large percentage of the austenite transforms to Martensite until the lower transformation temperature $\left(\mathrm{M}_{\mathrm{f}}\right)$ is reached, at which time the transformation is complete. For eutectoid steel, between 6 and $10 \%$ of the austenite, will remain, this known as retained austenite. The percentage of retained austenite is insignificant for percentages of carbon less than $0.6 \mathrm{wt} \%$ and increases to approximately $13 \mathrm{wt} \%$ at $0.95 \mathrm{wt} \% \mathrm{C}$. For a relatively high carbon steel $(1.4 \mathrm{wt} \% \mathrm{C})$ the retained austenite can be between $30-47 w t \%$. For Martensite to form, a very rapid quench is essential. For a eutectoid carbon steel $(0.78 \mathrm{wt} \% \mathrm{C})$ of thin section, if a quench starting at $750^{\circ} \mathrm{C}$ and ending at $450^{\circ} \mathrm{C}$ takes 0.7 seconds (a rate of $430^{\circ} \mathrm{C} / \mathrm{s}$ ) no Pearlite will form in the steel and the structure will be martensitic with small amounts of retained austenite. For steel $0-0.6 w t \% C$ the Martensite has the appearance of lath, and is called lath Martensite. Both the seamless and welded tubes used in this project will be from this group. For steel greater than $1 \mathrm{wt} \% \mathrm{C}$ it is called plate Martensite. Between those two, the physical appearance of the grains is a mix of the two. The strength of the Martensite is reduced as the amount of retained austenite grows. In the percentages between $0.6 \mathrm{wt} \%$ and $1.0 \mathrm{wt} \% \mathrm{C}$ the form will be a mixture of the two forms. If the cooling rate is slower than the critical cooling rate, some amount of Pearlite will form, starting at the grain boundaries where it will grow until the $M_{s}$ temperature is reached then the remaining austenite transforms into Martensite at about half the speed of sound in steel. In certain alloy steels, Martensite can also be formed by the deformation of the steel at temperature, in its austenitic form, by quenching to below $\mathrm{M}_{\mathrm{s}}$ and then working by plastic deformations to reductions of area to $40 \%$ or as little $20 \%$ of the original. The process produces dislocation densities up to $10^{13} / \mathrm{cm}^{2}$. The great number of dislocations, combined with precipitates that originate and pin the dislocations in place, produces an extremely hard steel. Thus, Martensite can be thermally induced or stress induced[24], [35].

One of the differences between the two phases, mentioned earlier, is that Martensite has a BCT crystal structure, whereas austenite has a FCC structure.[35] The transition between these two microstructures requires very little thermal activation energy because it is a diffusion-less process of transformation, which results in a rapid rearrangement of atomic positions, and has been known to occur even at cryogenic temperatures. Martensite has a lower density than austenite, so that the 
martensitic transformation results in an increase in relative volume. More importantly, the shear strain has a magnitude of about 0.26 and which determines the shape of the plates of Martensite.

Martensite and Bainite are not at equilibrium and therefore are not shown in the phase diagram of the iron-carbon system. Equilibrium phases form by slow cooling rates that allow sufficient time for diffusion, whereas Martensite, Bainite and acicular ferrite are usually formed with high cooling rates. Martensite forms as rejection of carbon is prohibited. It's forced to stay in the phase thereby distorting the crystal lattice and this strain makes the phase hard. Heating Martensite and allowing it to cool naturally produces tempered Martensite. Tempered Martensite has a good combination of hardness and ductility and therefore is more useful for many applications where a combination of both is required. Since quenching rates can be difficult to control, steels are often quenched rapidly to produce a surplus of Martensite; they are then tempered to gradually alleviate the stresses until the correct structure is achieved for the chosen application.[24], [36] 


\subsubsection{Bainite}

Bainite is an acicular microstructure (not a phase) that forms in steels at temperatures of $250-550^{\circ} \mathrm{C}$, depending on the chemical composition. Bainite was first described as a decomposition product that can be formed when austenite is cooled past the critical temperature. Davenport and Bain described the microstructure as being comparable in appearance to tempered Martensite. Bainite has a fine, non-lamellar structure and commonly consists of cementite and dislocation-rich ferrite. The high concentration of dislocations present in the $\alpha$-iron means that it is harder than standard ferrite.

The temperature range for production of Bainite is between those for Pearlite and Martensite. When formed during continuous cooling, the cooling rate to form Bainite is not as severe as in the production of Martensite, but more rapid than that the formation of Pearlite in steels of the same composition. It is normally observed when the quenching rate is not great enough for a thick section. The addition of various alloying elements can increase the rate of Bainite formation by lowering the temperature required for its formation. The most effective alloying element for this is carbon.

The similarity in appearance between the microstructures of Martensite and Bainite is renowned as the two materials share many aspects of their transformation mechanisms. This means it difficult to distinguish one from the other as they both utilize displacement of carbon rather than diffusion. However, differences do exist. Under a light microscope, the microstructure of Bainite appears darker than Martensite. This darker appearance is due to its poor light reflectivity. Differences can also be seen when high resolution electron microscopy is used.

Bainite, much like its formation temperature is an intermediate of Pearlite and Martensite in terms of hardness. For this reason, the bainitic microstructure becomes useful in that no additional heat treatments are required after initial cooling to achieve a hardness value between that of Pearlitic and martensitic steels. However, Bainite has a very low initial yield point. This means that it has a low yield to ultimate tensile stress ratio meaning that it plastically deforms from relatively low stresses when compared to the final failure. Tempering of Bainite can give a good compromise of hardness and ductility. 


\subsubsection{Acicular Ferrite}

Acicular ferrite crystals have a lenticular shape but the bulk material is characterised in two dimensions by its needle-like grain structure. The chaotic microstructure increases the materials toughness. Acicular ferrite is formed by direct nucleation on the inclusions present in austenite grains which results in short, randomly oriented ferrite needles. Acicular ferrite is also characterised by high angle boundaries between the grains which reduces the likelihood of crack propagation through cleavage. Acicular ferrite is a fine Widmanstätten constituent, and is nucleated by an optimum intragranular dispersion of oxide, sulphide or silicate particles.

Due to the toughness of acicular ferrite, if a material is being welded, the composition of the material or the filler metal is often controlled in order to maximise the volume fraction of acicular ferrite. Higher alloying concentration and faster cooling delay transformation during continuous cooling, causing the austenite to convert to acicular ferrite at lower temperatures. This reduced temperature can be below the Bainite start temperature which increases the hardness of the material[29]. The effectiveness of inclusions as nucleation sites in modern low alloy steel weld metals is such that fine intragranular Bainite can nucleate on them, both by continuous cooling and by isothermal transformation below the Bainite start temperature. Acicular and Widmanstätten ferrite can be mistaken for intragranular Bainite when observed using at optical microscope.[14] 


\subsection{Crystallographic Theory}

\subsubsection{Crystal Structures}

The physical structure of solid materials depends upon the arrangement of the constituent atoms, ions or molecules and the interactions between them. Metals have a crystalline structure that is repeated in three dimensions. This is knows as long range order. The atomic arrangement of a crystalline solid can be explained by comparing an atoms position in the lattice to a point on intersection on a network of lines in three dimensional space.[35] The lattice on which the atoms sit is (theoretically) an infinite three dimensional array of points. Each point in the lattice is surrounded by the same grouping of identical lattice points. Therefore, each lattice space can be described by specifying the position of each atom in a repeating unit cell. A unit cell is the smallest subdivision of a lattice that still maintains the characteristics of the overall crystal lattice. The positions of the atoms in a unit cell are defined from the corner of the cell with the $a, b$ and $c$ lattice vectors. The axial lengths of the unit cell and the inter-axial angles $\alpha, \beta$ and $\gamma$ are known as the lattice constraints. The literature states that there are only seven unit cells needed in order to construct all of the known space lattices. These are: Cubic, Tetragonal, Orthorhombic, Rhombohedral, Hexagonal, Monoclinic and Triclinic. Most of the crystal systems are variations of the basic unit cells. There are fourteen possible crystal lattices which are categorised under four main sub-divisions: Simple, body-centred, face centred and base-centred.

\subsubsection{Body Centred Cubic (BCC)}

Body centred cubic is the lattice form for iron at ambient temperature. The BCC structure unit cell has the equivalent of 2 atoms contained within it. One atom at the centre of the cell and eight eighths; one at each corner of the cell. The atoms that contact each other in a BCC unit cell across the cube diagonal. The relationship between the length of the cube side, $a$ and the atomic radius, $R$ is:

$$
\alpha=\frac{4 R}{\sqrt{3}}
$$

\section{Equation 9-BCC relationship between the lattice constant, $a$ and the atomic radius, $R$}

The unit cell length, a for BCC iron is $0.2864 \mathrm{~nm}$. The atomic packing factor of the BCC unit cell is relatively low at 0.68 or $68 \%$. The atomic packing factor (APF) is effectively the density of the atoms within it and is calculated by dividing the volume of the atoms in the unit cell by the volume of the total BCC unit cell: 


$$
A P F=\frac{\text { Volume of atoms in BCC unit cell }}{\text { Volume of BCC unit cell }}
$$

Where

$$
\begin{gathered}
V_{\text {atoms }}=(2)\left(\pi R^{3}\right)=8.373 R^{3} \\
V_{\text {unit cell }}=a^{3}=12.32 R^{3} \\
\therefore A P F=\frac{V_{\text {atoms }}}{V_{\text {unit cell }}}=\frac{8.373 R^{3}}{12.32 R^{3}}=0.68
\end{gathered}
$$

Equation 10-Atomic packing factor for BCC unit cell

Thus, $68 \%$ of the BCC unit cell is occupied by atoms. The BCC structure is loosely packed lattice.
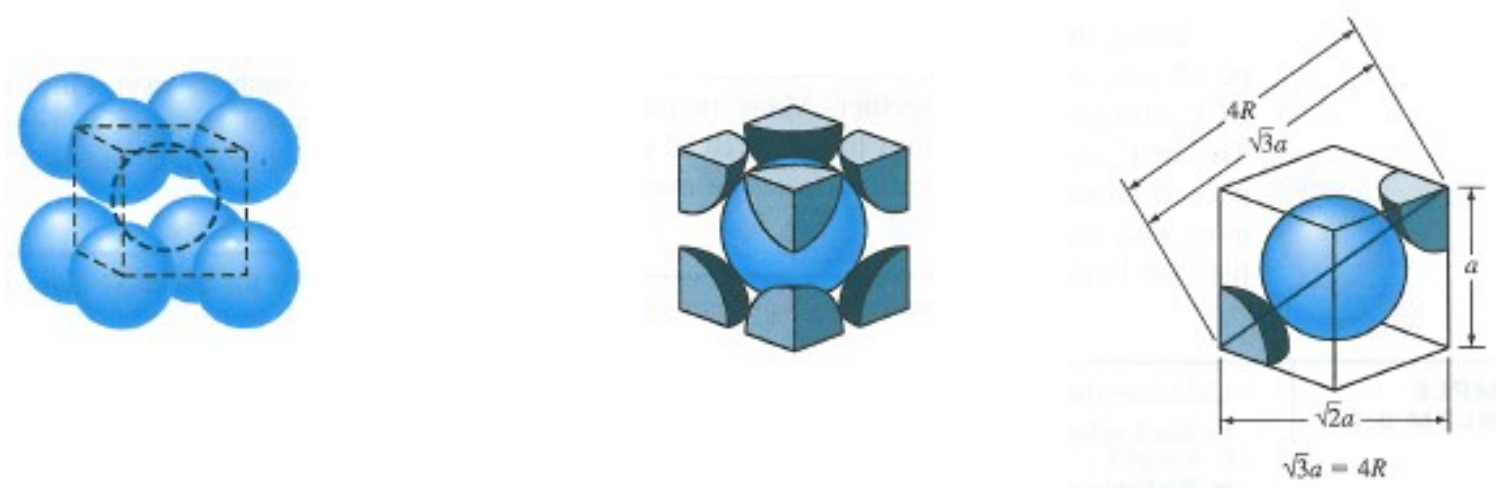

Figure 12-(left to right): Schematic BCC hard-sphere unit cell: Schematic BCC isolated unit cell: Schematic diagram of an BCC isolated unit cell showing the relationship between the lattice constant, $a$ and the atomic radius, $R[35]$ 


\subsubsection{Face Centred Cubic (FCC)}

This crystal structure has a unit cell of cubic geometry, with atoms located at each corner and the centre of each face. Copper aluminium, gold and silver all have FCC crystal structures. Austenite or $\gamma$-iron also has an FCC structure. The atoms in an FCC structure are packed together as closely as possible. The atomic packing factor for this structure is 0.74 which is significantly greater than that of the BCC unit cell. FCC contains the equivalent of four atoms per unit cell. One atom split into eight octants at the corners and a further three atoms split into halves at the faces. The atoms in an FCC structure contact each other across the cubic face diagonal thus showing the relationship between the length of the cubic side, a and the atomic radius is:

$$
a=\frac{4 R}{\sqrt{2}}
$$

Equation 11-FCC relationship between the lattice constant, a and the atomic radius, $\mathbf{R}$
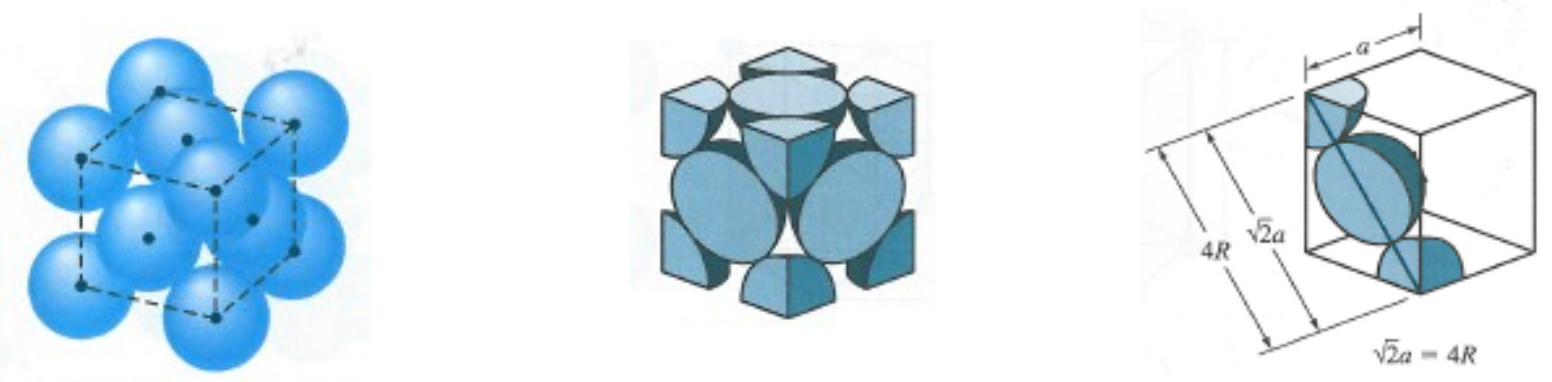

Figure 13-(left to right): Schematic FCC hard-sphere unit cell[35]: Schematic FCC isolated unit cell[35]: Schematic diagram of an FCC isolated unit cell showing the relationship between the lattice constant, $a$ and the atomic radius, $R[35]$ 


\subsubsection{Crystalline Imperfections}

The concept of crystalline defects was introduced in the 1930's by Taylor, Orowan and others to explain the behaviour of crystalline materials during deformation. Later work on metals indicated the dependence of the mechanical properties on the presence of crystalline defects such as dislocations.[10] Work hardening theories were developed on the basis of dislocation interactions with obstacles such as grain boundaries, precipitates and other dislocations within the material.

In reality, crystals are never perfect and contain a varying number of imperfections that affect the mechanical properties of the material. The imperfections of a crystal lattice are classified according to their shape. These classifications include; point, line, planar and volume defects.

\subsubsection{Point Defects}

The simplest point defect is a vacancy. A vacancy is an atom site from which an atom is missing. Vacancies can be produced during solidification as a result of disturbances during crystal growth. In metals, the equilibrium concentration of vacancies is approximately 1 in 10,000 atoms. Additional vacancies can also be introduced into a metal by plastic deformation among other methods including rapid cooling from an elevated temperature. Non-equilibrium vacancies have a tendency to cluster, producing di or tri-vacancies. Vacancies can move through the material by exchanging position with their neighbours. This movement is particularly apparent at elevated temperature where the atomic mobility is greater.[35] The other point defect is known as an interstitial. An interstitialcy is where at atom can occupy the interstitial space between other atoms. This defect does not usually occur naturally due to the local deformation they cause; however, can be introduced using irradiation. Impurity additions of the substitutional or interstitial type are also point defects and may be present in metallic or covalently bonded crystals Carbon as an alloying addition in steel is a good example of an interstitial atom.

\subsubsection{Line Defects}

Line imperfections or dislocations are defects that cause distortion of the crystal lattice that is centred on a line. Dislocations are produced during solidification of the material and are often formed during plastic deformation of the crystal lattice. The two main varieties of dislocation are edge and screw types. There are also combinations, known as mixed dislocations. 


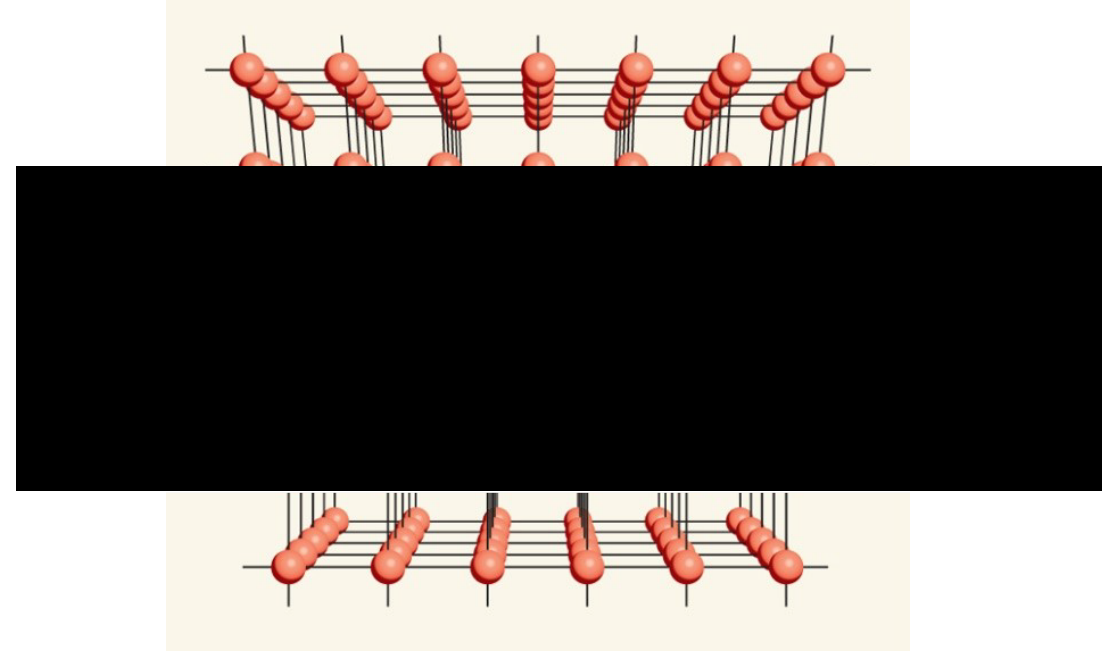

Figure 14-Schematic showing positive edge dislocation of a crystal lattice at atomic resolution[37]

An edge dislocation is formed by the insertion of an extra half plane of atoms. This can be seen in Figure 14. The displacement distance between the atoms around the dislocation is called the slip vector[35], [37] and is perpendicular to the edge-dislocation line. Dislocations are not equilibrium defects and therefore store energy in the distorted region of the lattice around the dislocation. An edge dislocation has a region in compression in the half plane and a region in tension below the half plane.

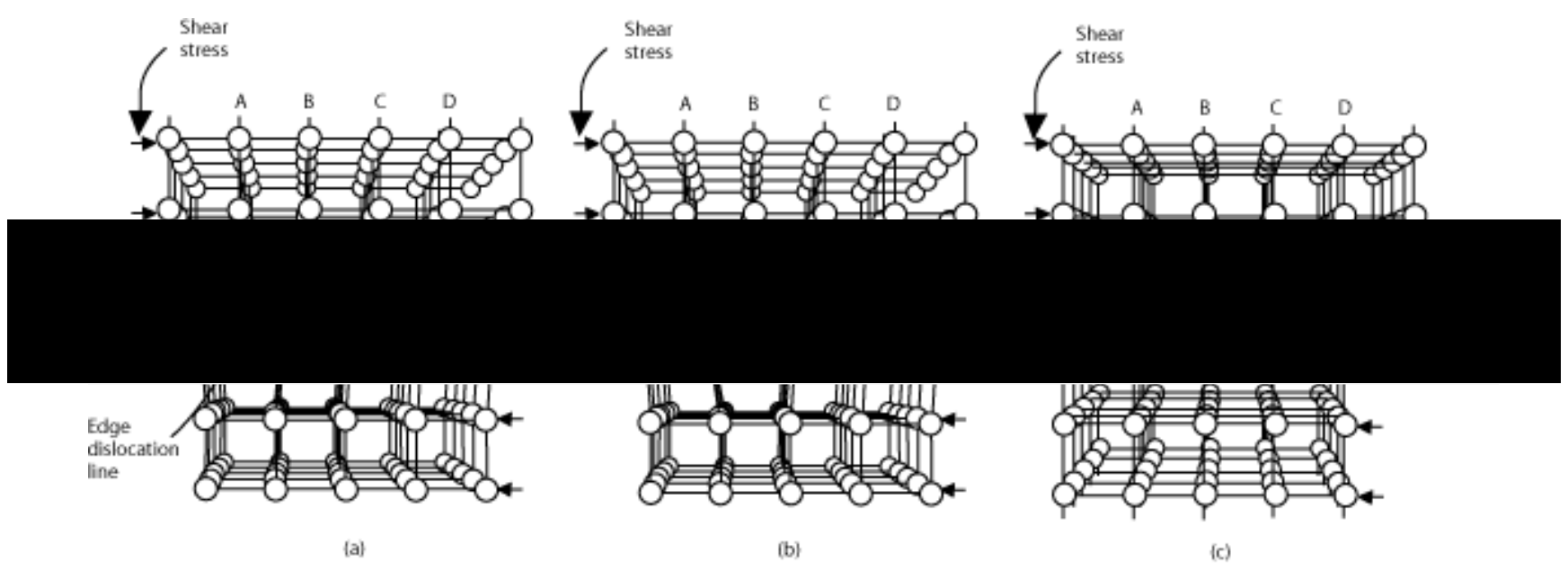

Figure 15-Movement of an edge dislocation through a crystal lattice[63]

As shown in Figure 15, the dislocation moves one plane at a time is discreet steps. The dislocation in the top half of the crystal is moving to the right from its position shown in Figure 15 (a) to its position in Figure 15(b) and finally Figure 15 (c). In the process of slipping one plane at a time the dislocation propagates across the crystal. The movement of the dislocation across the plane 
eventually causes the top half of the crystal to move with respect to the bottom half. However, only a small fraction of the bonds are broken at any given time. Movement in this manner requires a much smaller force than breaking all the bonds across the middle plane simultaneously.

A screw dislocation can be formed in a crystal by applying differential shear stress either side of a cutting plane. These shear stresses introduce a distortion in the crystal lattice in the form of a spiral ramp. This can be seen in Figure 16. The lower right image shows the plane of atoms just above the rip. The atoms represented by the blue circles have not yet moved from their original position. The atoms represented by the red circles have moved to their new position in the lattice and have reestablished metallic bonds. The atoms represented by the green circles are in the process of moving. It can be seen that only a portion of the bonds are broken at any given time. As is the case with the edge dislocation; movement in this manner requires a significantly smaller force than shearing all of the bonds along the middle plane simultaneously. 


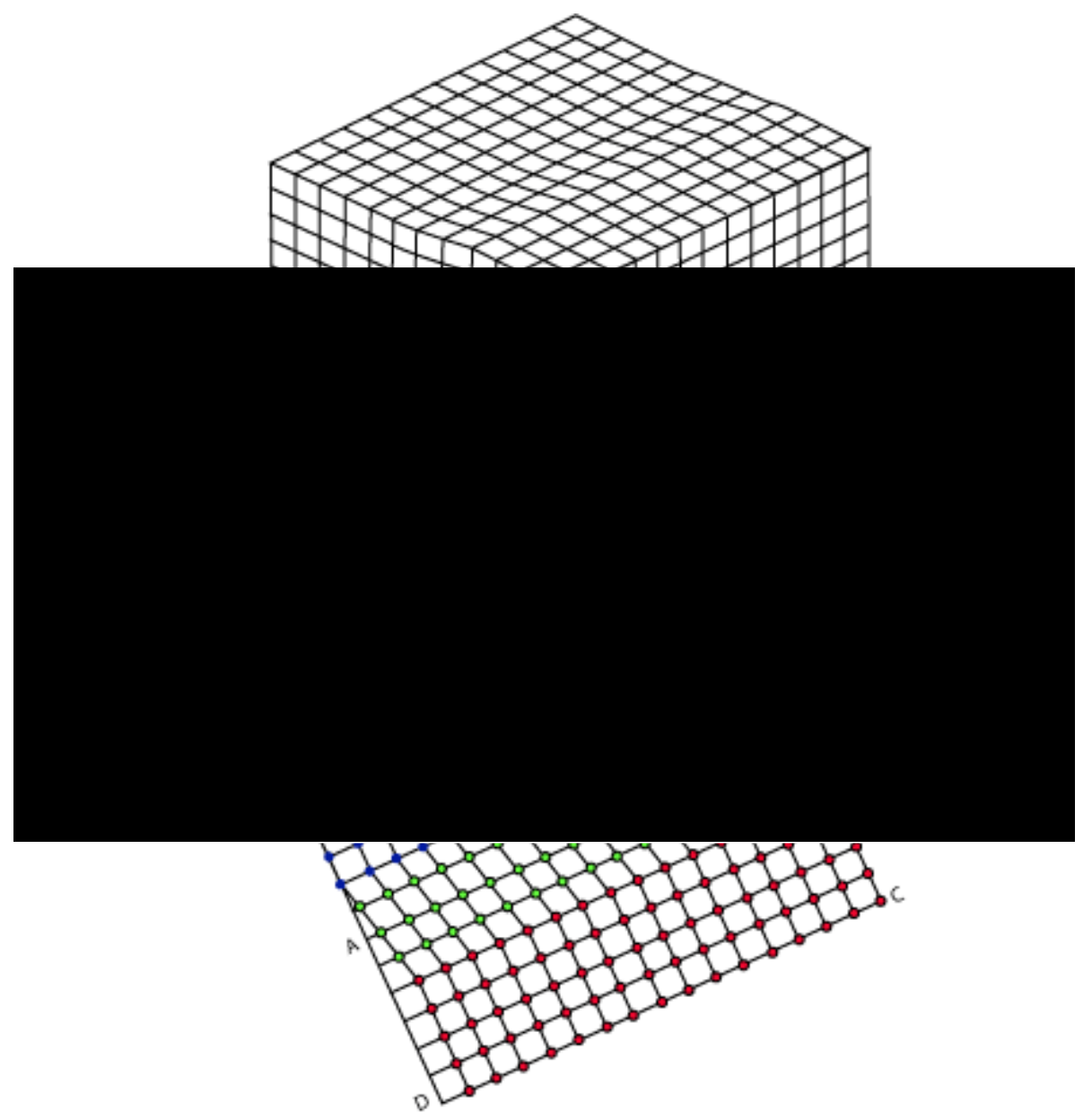

Figure 16-Screw dislocation schematic of a crystal lattice at atomic resolution[63] 


\subsubsection{Planar Defects}

Planar defects include external surfaces, grain boundaries, low and high-angle boundaries, twins twists and stacking faults. The external surface of any material is the most common planar defect. External surfaces are considered defects because the atoms on the surface are only bonded to atoms on one side and therefore have a lower number of neighbours. This results in a higher energy state at the surface when compared to atoms inside the lattice with the correct number of neighbouring atoms. This higher energy at the surface makes the material more susceptible to erosion and reaction with the environment.

Grain boundaries are imperfections that separate two crystals with different orientations. The grain boundaries in a metal are created during solidification. The crystals start at separate nuclei and grow simultaneously until the crystals meet at the grain boundaries. The shape of a grain is determined by the restriction imposed upon it by other grains surrounding the crystal. The grain boundary it-self is narrow, between 2 and 5 atomic diameters. There is an atomic mismatch that exists due to the differing orientation of the crystal lattices. This atomic mismatch leads to a lower atomic packing and higher energies. The higher energy at the grain boundaries along with the relatively large spacing produces a more favourable environment for growth of precipitates and nucleation. Grain boundaries restrict plastic flow by restricting dislocation movement in the boundary region. Thus, materials with a finer grain structure and therefore; more grain boundaries per unit volume tend to be stronger. This restriction of dislocations is of particular importance for cold bending of steel sections. The ease of dislocation movement in any direction will affect the mechanical properties of the section. Grain boundaries are the most effective two dimensional defect for strengthening a material.

Twin boundaries are formed when the material is permanently plastically deformed. Therefore, they are likely to be produced during both the cold-formation of the tubes and cold-roller bending. Twist boundaries can also be formed during re-crystallising process. This happens when the atoms reposition themselves within a deformed crystal at elevated temperature. However, for the materials studied within this project it is unlikely to have occurred. As with dislocations, twin boundaries tend to strengthen a material.

Sometimes during the production of a crystalline material interaction of dislocations or collapse of a vacancy cluster can lead to a stacking fault. Stacking faults usually occur in FCC and HCP crystal structures and usually restrict the movement of dislocations and therefore strengthen the material. 


\subsubsection{Volume Defects}

Volume defect are three dimensional defects usually form when a cluster of point defects join to form a void in the lattice. However, a collection of impurity atoms or alloying additions may join to form a precipitate. The size of these defects can vary significantly. These defects have a significant effect on the mechanical performance of the material.

\subsection{Deformation}

\subsubsection{Uniaxial Plastic Deformation}

To initiate deformation by slip, dislocations must be moved or generated and then moved [38]. The dislocations present in the virgin material become secured by obstacles such as precipitates or other dislocations. The stress of the upper yield point is required in order to release them from the "blockages". However, after the dislocations start to move, the stresses needed to propagate dislocation movement is reduced. This reduction in required energy is the cause of the lower yield point in steel. After the lower yield point, dislocations continue to glide without any increase in stress for a period of increased strain. However, eventually they will meet with obstacles and the stress required to continue motion is increased as the dislocations start to stack up. If the section is unloaded and then reloaded at ambient temperature the dislocations will continue to move along the same path and the upper yield point will not be observed.

The significant majority of engineering alloys are poly-crystalline with the exception of single grain nickel super alloys for use in jet engine rotor blades among a few others. Grain boundaries strengthen metals and their alloys by restricting the movement of dislocations at ambient temperature. However at high temperatures they become areas of weakness. A fine grain size is desirable for materials that require high strength or toughness. However, they are more susceptible to creep and corrosion.[35] A fine, equiaxed grain structure will have uniform and isotropic mechanical properties. During plastic deformation of metals, dislocations move along slip planes and cannot usually move intra-granularly. Slip lines change direction at grain boundaries and therefore, each grain has its own dislocations moving along slip planes which have different orientations to the adjacent grains. As the number of grains increases, the size of the grain becomes smaller. This means that dislocations within the grains can travel a shorter distance before being stopped at a boundary. This causes dislocation pile-up. Dislocation pile-up is the reason that finer grained materials to have a higher strength when compared to a courser grain equivalent. The process of dislocations piling up caused the material to become more resistant to further strain in the direction of the flow stress. This process is known as work hardening. The shape of the grain is 
altered with plastic deformation. The grains are sheared relative to each other by the generation and movement of dislocations. The distortion of the grains leads to an elongated microstructure. Dislocation density increases with cold-deformation. New dislocations are generated and interact with existing dislocations increasing the dislocation density. As the density of the dislocations increases it becomes more difficult for the dislocations to move further and therefore, the material work hardens in line with plastic deformation. The ductility of the material is decreased with coldwork and therefore, the elongation of the material will decrease when tested. Strain hardening is one of the most prominent methods for strengthening a metal and the strength increase can easily be controlled by varying the amount of strain that the material experiences during production. 


\subsection{Heat Treatments of Steel}

It is the specific microstructure and chemistry of a steel that determines its mechanical properties. Even slight changes in either the microstructure or the chemical composition can have marked effects on the yield strength, hardness, etc.[24] There are a number of ways to alter the microstructure of steel without consciously altering the chemical composition. The main method is controlling the rate at which the steel cools from austenite temperature. This can be done by altering the cooling rate of the steel during initial production or by performing heat treatments on the steel after it has been fully formed.[31] The cooling rate determines the grain size of the steel; it also has some control over the variety of unit cell that makes up the steel. For example, quenching (rapid cooling rate performed by water being sprayed onto the hot metal) from above the critical temperature can produce Martensite. If the steel is cooled slowly, diffusion of carbon is allowed. This produces a structure called Pearlite

A heat treatment can be performed as the steel is initially produced, or later in its processing. The three main heat treatments are normalising, annealing and tempering. In normalising, the steel is allowed to cool from an elevated temperature (above the critical), under normal atmospheric conditions or "still air" [24], [35] In annealing, the steel is cooled from a high temperature in a controlled atmosphere. The atmosphere is controlled to prevent corrosion and is often inert or partially reducing[39], [40]. The rate of cooling is controlled by a furnace. These processes allow a longer period of grain growth; producing larger crystal structures, giving different mechanical properties.

\subsubsection{Annealing}

Annealing is a heat treatment that alters a material to increase its ductility and formability. In steel it involves heating the material to above its critical temperature, then "soaking" at this elevated temperature, before cooling.[41]

Annealing is used to increase ductility; it softens the material by relieving internal stresses. The process homogenises the material, refining the structure, which improves the properties for cold working.

In the cases of copper, steel, silver, and brass, this process is performed by heating the material for a prolonged period called the soaking time before cooling to ambient conditions. Cooling is usually done in the presence of an inert or reducing gas[23]. Copper, silver and brass can be cooled slowly in air, or quickly using a water quench. However, ferrous metals, such as steel, must be cooled slowly to anneal. The metal is softened and prepared for further work, such as; shaping, stamping, 
or forming. Annealing can be done in a batch process or in the case of strip products, can be performed continuously.

\subsubsection{Normalising}

Normalisation is a cooling process applied to ferrous alloys such as steel to give the material a uniform fine grained structure and make it less brittle. It is used for steels with carbon concentrations $<0.4 \mathrm{wt} \%$ to transform austenite into ferrite, Pearlite and sorbite (a finer lamella structure than standard Pearlite). The process involves heating the section above the critical temperature. It is then soaked for a short period at this temperature before being allowed to cool in air. Smaller grains form producing a tougher, more ductile material. It eliminates columnar grains and dendritic segregation that sometimes occurs during casting.[23] Normalizing improves machinability of a component and provides dimensional stability if subjected to further heat treatment processes. The hot-finished sections used in this project have been normalised.

\subsubsection{Tempering}

Tempering is a process of heat treating, which is used to increase the toughness of iron-based alloys. Tempering is usually performed after cooling, to reduce some of the excess hardness, and is done by heating the metal to a much lower temperature than is required for recrystallisation.[13] The choice of temperature depends on both the specific composition of the alloy and on the desired properties in the finished product. For example, very hard tools are often tempered at low temperatures, while springs are tempered at much higher temperatures.

Tempering is most often performed on steel that has been heated above its critical temperature before quenching. Quenching can be performed using methods such as immersing the red-hot steel in water, oil, or forced-air. The quenched-steel produced is in its hardest possible state, which is fully martensitic if the cooling is rapid enough. It is then tempered to decrease the hardness to a point more suitable for the desired application. The hardness of the quenched-steel depends on both cooling speed and on the composition of the alloy.[12], [28][13] Steel with a high carboncontent or carbon equivalent value (CEV) will become much harder than a steel with a low carboncontent. Likewise, tempering high-carbon steel to a certain temperature will produce steel that is considerably harder than low-carbon steel that is tempered at the same temperature. The amount of time held at the tempering temperature also has an effect. Tempering at a slightly elevated temperature for a shorter time may produce the same effect as tempering at a lower temperature for a longer time. Tempering times vary, depending on the carbon content, size, and desired application of the steel, but typically range from minutes to hours. 
Tempering quenched-steel at very low temperatures, between 66 and $148^{\circ} \mathrm{C}$, will usually not have much effect other than a slight relief of some of the internal stresses. Tempering at higher temperatures, from 148 to $205^{\circ} \mathrm{C}$, will produce a slight reduction in hardness, but will primarily relieve much of the internal stresses. Tempering in the range of 260 to $340^{\circ} \mathrm{C}$ causes a decrease in ductility. This is known as a ductility trough and can increase the brittleness, and occurs in the "tempered Martensite embrittlement" range. Steel requiring more strength than toughness, such as tools are not usually tempered above $205^{\circ} \mathrm{C}$. Instead, a variation in hardness is usually produced by varying only the tempering time. When increased toughness is desired at the expense of strength, higher tempering temperatures, from 370 to $540^{\circ} \mathrm{C}$, are used $[13,19]$. Tempering at even higher temperatures, between 540 and $600^{\circ} \mathrm{C}$, will produce excellent toughness, but at a serious reduction in the strength and hardness. At $600^{\circ} \mathrm{C}$, the steel may experience another stage of embrittlement, called "temper embrittlement", which occurs if the steel is held within the embrittlement temperature range for too long. When heating above this threshold, the steel will not be held for a prolonged period, and quickly cooled to avoid temper embrittlement. 


\subsubsection{Reverse Plastic Deformation}

During reverse deformation the dislocation density has been found to decrease when compared to the initial strain direction. This has been observed in both BCC and FCC structures. [10], [18], [42],[15]. This reduction leads to a uniform distribution of dislocation density just after the load reversal. The cell structure is restored on further reverse strain. With an increase in forward prestrain the cell structure dissolution strain is increased during reverse deformation.[43]

Investigation of dislocation structure change during strain path change of less than $180^{\circ}$ also showed dissolution of the cell walls in both BCC and FCC materials[44], [45]. However, in highly alloyed materials dislocation structure change with strain path change has not been observed, which was related to dislocation immobilisation by precipitates after pre-straining[45]. In addition to the reduction of the yield strength observed in a pure Bauschinger test where the strain path is changed by $180^{\circ}$, strain path changes of less than $180^{\circ}$ also leads to yield reduction.[45] This is of particular importance for this project as the material is curved and not just axially strained. This has a high industrial importance, as pure Bauschinger conditions are observed less frequently than strain path changes of less than $180^{\circ}$. This must be taken into account in design calculations where cold-forming is used. 


\subsubsection{Bauschinger effect and work-softening}

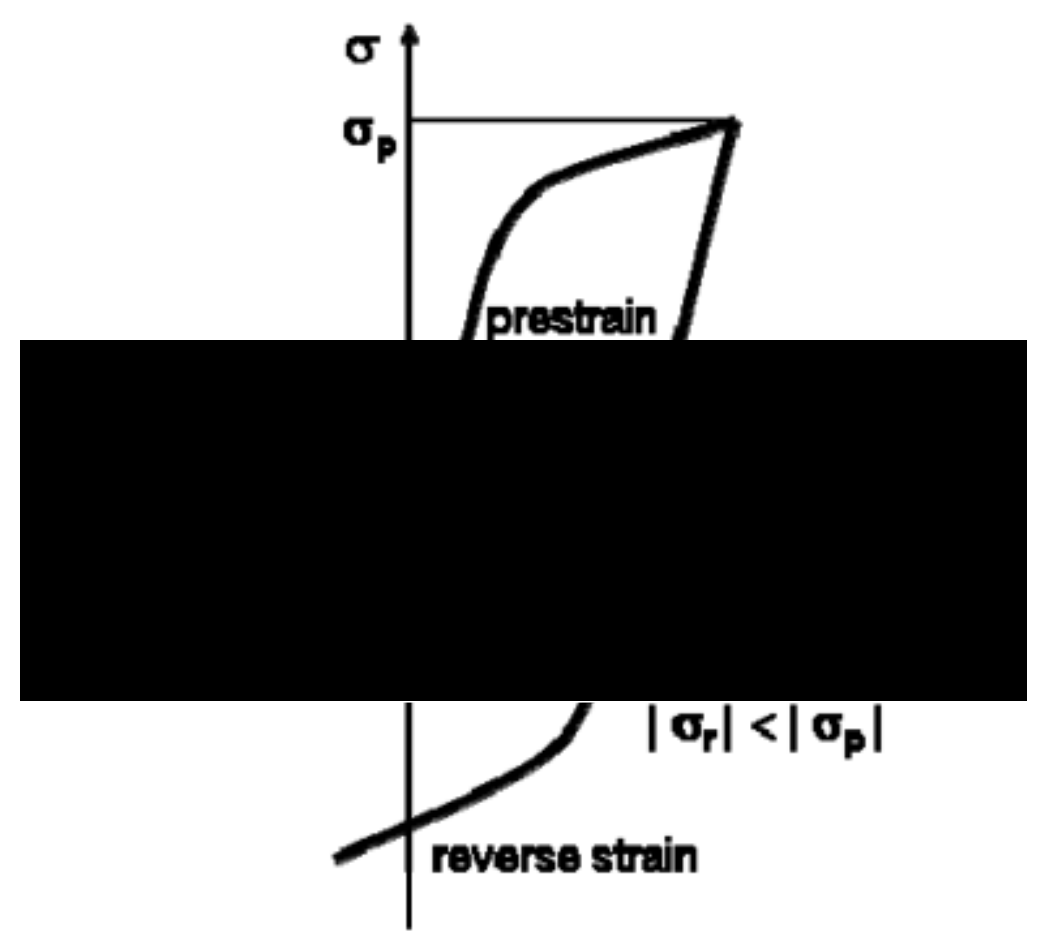

Figure 17-Schematic diagram of the Bauschinger effect where $\sigma_{p}=$ maximum pre-stress, $\sigma_{r}=$ the yield stress in the reverse direction [35]

According to the literature[46]-[48], there are three parameters that are used to assess the absolute values of the Bauschinger effect. These are the stress, the strain and energy. This can be seen in Figure 18. There are a number of different mathematical expressions for the stress parameter alone. Each is related to different points on the forward-reverse stress-strain curve. The stress parameter $\beta_{\sigma 1}$ describes the relative decrease in the yield stress from the forward to reverse deformation:

$$
\beta_{\sigma 1}=\frac{\sigma_{p}-\sigma_{r}}{\sigma_{p}}
$$

Equation 12-Relative decrease in yield stress from forward to reverse deformation

Where $\sigma_{p}$ is the maximum pre-stress and $\sigma_{r}$ is the yield stress in the direction of reverse strain[10] 
The stress parameters $\beta_{\sigma 2}, \beta_{\sigma 3}$ and $\beta_{\sigma 4}$ are calculated using stress values at both $0.2 \%$ and $0.5 \%$ reverse strain.

$$
\beta_{\sigma 2}=\frac{\sigma_{p}-\sigma_{r 0.2}}{\sigma_{p}}
$$

Equation 13-Property restoration during reverse straining after the yield drop at $0.2 \%$ reverse strain

Where $\sigma_{r 0.2}$ is the stress at $0.2 \%$ reverse strain

$$
\beta_{\sigma 3}=\frac{\sigma_{p}-\sigma_{r 0.5}}{\sigma_{p}}
$$

Equation 14-Property restoration during reverse straining after the yield drop at $0.5 \%$ reverse strain Where $\sigma_{r 0.5}$ is the stress at $0.5 \%$ reverse strain

$$
\beta_{\sigma 4}=\frac{\sigma_{r 0.5}}{\sigma_{p}}
$$

Equation 15-Property restoration during reverse straining after the yield drop

As yielding represents the initiation of dislocation movement, parameter $\beta_{\sigma 1}$ defines the "short range" work-softening. As previously discussed, the rate of work-hardening depends on the chemistry of the material such as the alloying additions and the microstructure, as well as the phases present, precipitates and the dislocation density. The "long-range" work-softening is defined by the remaining three parameters, $\beta_{\sigma 2}, \beta_{\sigma 3}$ and $\beta_{\sigma 4}$. They define how the Bauschinger effect affects the stress to strain relationship as deformation increases in the reverse direction.

The strain parameter describes the relative strain rate in the reverse direction needed to achieve the same change in length produced during pre-strain:

$$
\beta_{\varepsilon}=\frac{\varepsilon_{r}}{\varepsilon_{p}}
$$

\section{Equation 16-Bauschinger strain parameter}

Where $\varepsilon_{p}$ is the plastic strain and $\varepsilon_{r}$ is the reverse plastic strain for the point of equal modulus stress to that of the pre-stress 


$$
\beta_{E}=\frac{E_{S}}{E_{p}}
$$

\section{Equation 17-Bauschinger energy parameter}

Where $E_{p}$ is the energy spent during pre-strain and $E_{s}$ is the energy saved during reverse strain due to the Bauschinger effect

The Bauschinger strain and energy parameters also gave an indication of the "long-range" Bauschinger effect. The "long-range" parameters give an indication of how long lasting the Bauschinger effect is. As the dislocations start to stack up in the opposite direction as reverse strain is increased, the material will start to harden in the same way as the pre-strain phase. It is the

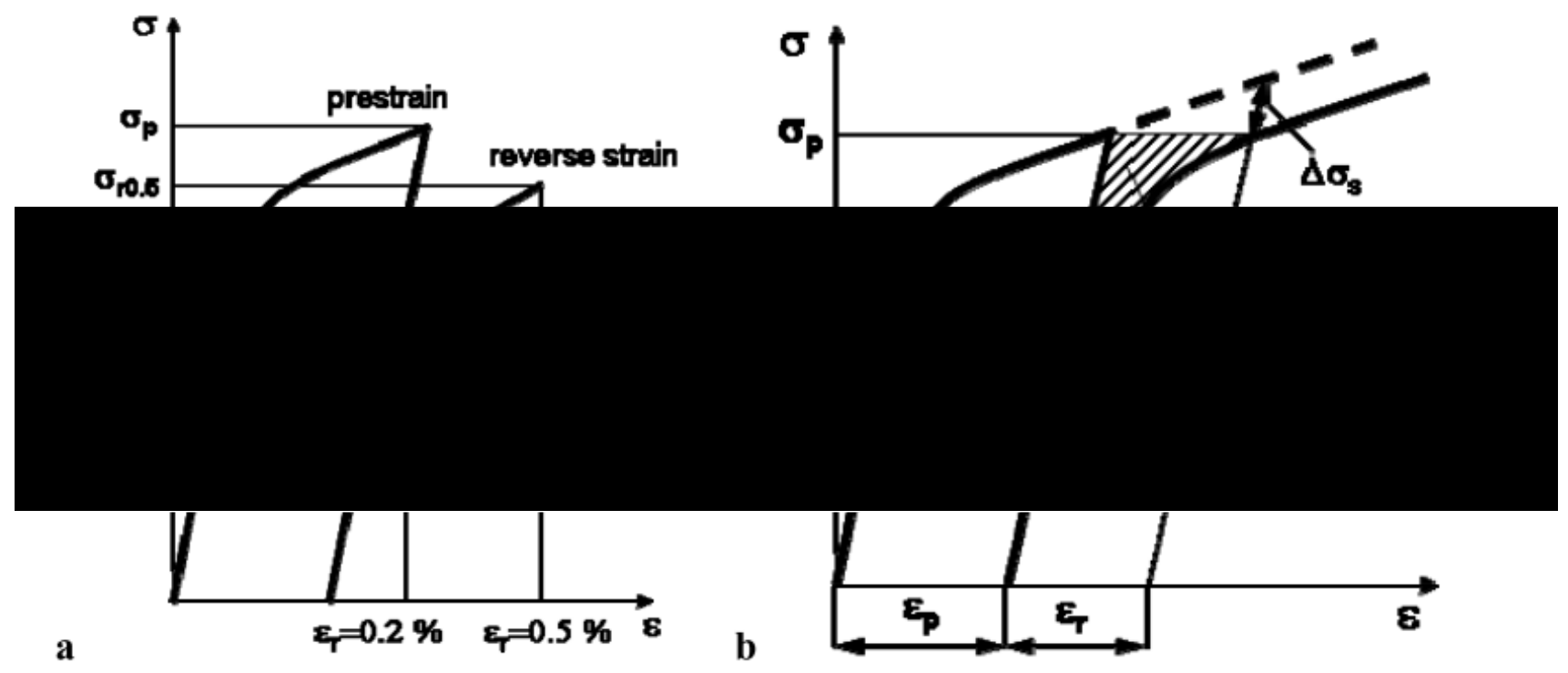

Figure 18-Modulus stress-strain curves for (a) the Bauschinger effect stress-parameter and (b) the Bauschinger effect strain and energy parameters[10]

Where: $\sigma_{\mathrm{r} 0.2}$ and $\sigma_{\mathrm{r} 0.5}-$ the yield stress at $0.2 \%$ and $0.5 \%$ reverse strain

$\Delta \sigma_{\mathrm{s}}=$ permanent work-softening

$\varepsilon_{p}=$ pre-strain

$\varepsilon_{r}=$ "Bauschinger strain", the strain in the reverse direction corresponding to the point of reverse stress equal to the maximum pre-stress $\sigma_{p}$

$E_{p}=e n e r g y$ spent during pre-strain

$E_{s}=e n e r g y$ saved during reverse straining due to the Bauschinger effect, shown by the shaded area

difference in stress and strain required to reach the same strength in the reverse direction that is important. 


\subsubsection{Features of the Bauschinger effect}

As previously discussed, the Bauschinger effect leads to work softening in a material. With an increase in pre-strain the stress parameter $\beta_{\sigma 1}$ increases and the strain and energy parameters decrease. This is likely related to the total dislocation density increase. This leads to an increase in the yield lowering effect. However the mobility of dislocations are increased, leading to a faster return of strength as pre-strain is increased. Abel and Muir reported yielding during unloading and as a consequence of this, it was shown that in some cases $\beta_{\sigma 1}>1$.[46] This was explained by Abel and Muir by relaxation of the elastic back stresses in some grains led to plastic deformation of the adjacent grains in some cases.

The appearance of a plateau on the reverse stress-strain curve shown in Figure 20 is related to the dislocation density decrease and the cell structure dissolution during the initial stage of reverse loading. This has been observed in both FCC and BCC materials.[49] With an increase in reverse strain the motion of the dislocations are once again impeded leading to an increase in dislocation density and therefore an increase in stress-strain curve gradient.

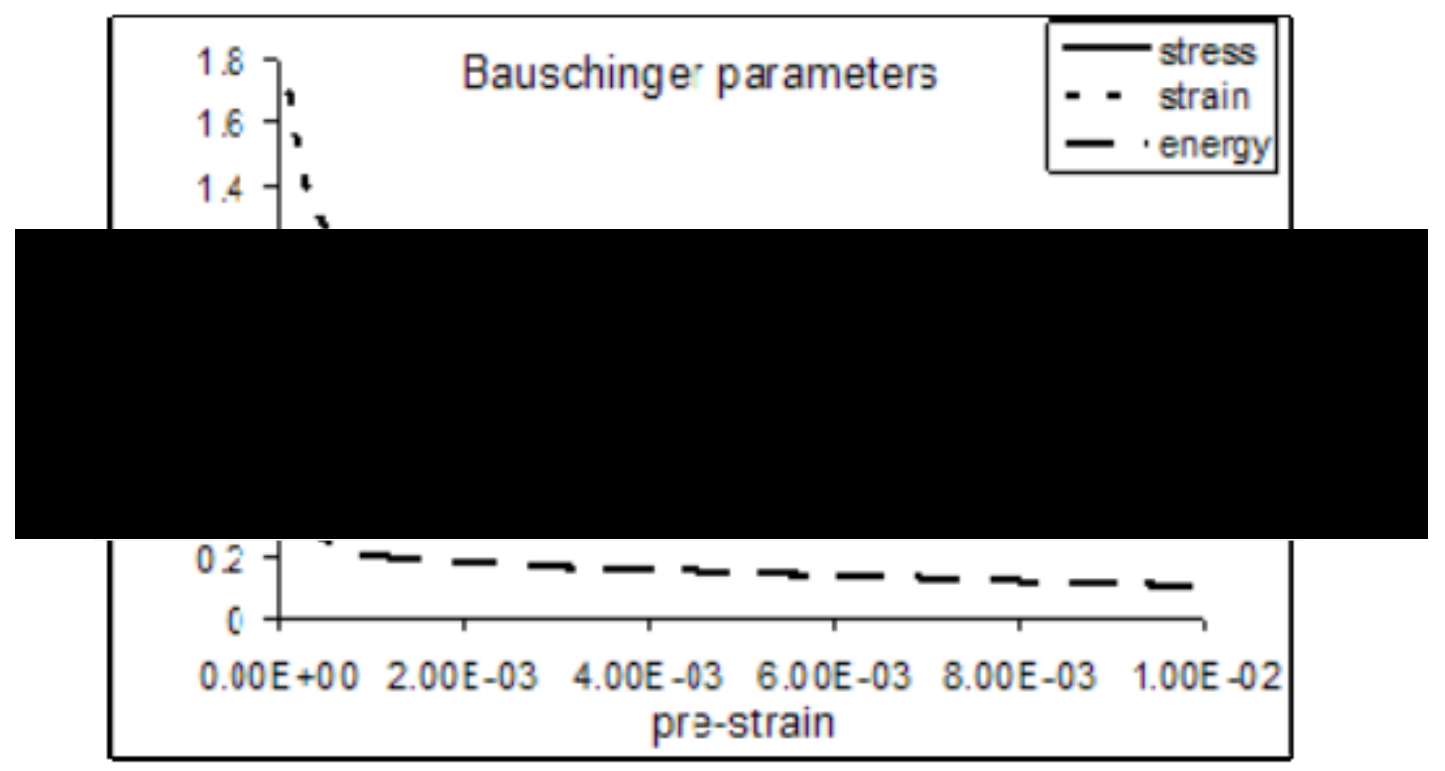

Figure 19-The Bauschinger effect parameters dependence on pre-strain for $0.17 \%$ C steel derived by Kostryzhev from work by Muir and Abel[10], [46] 


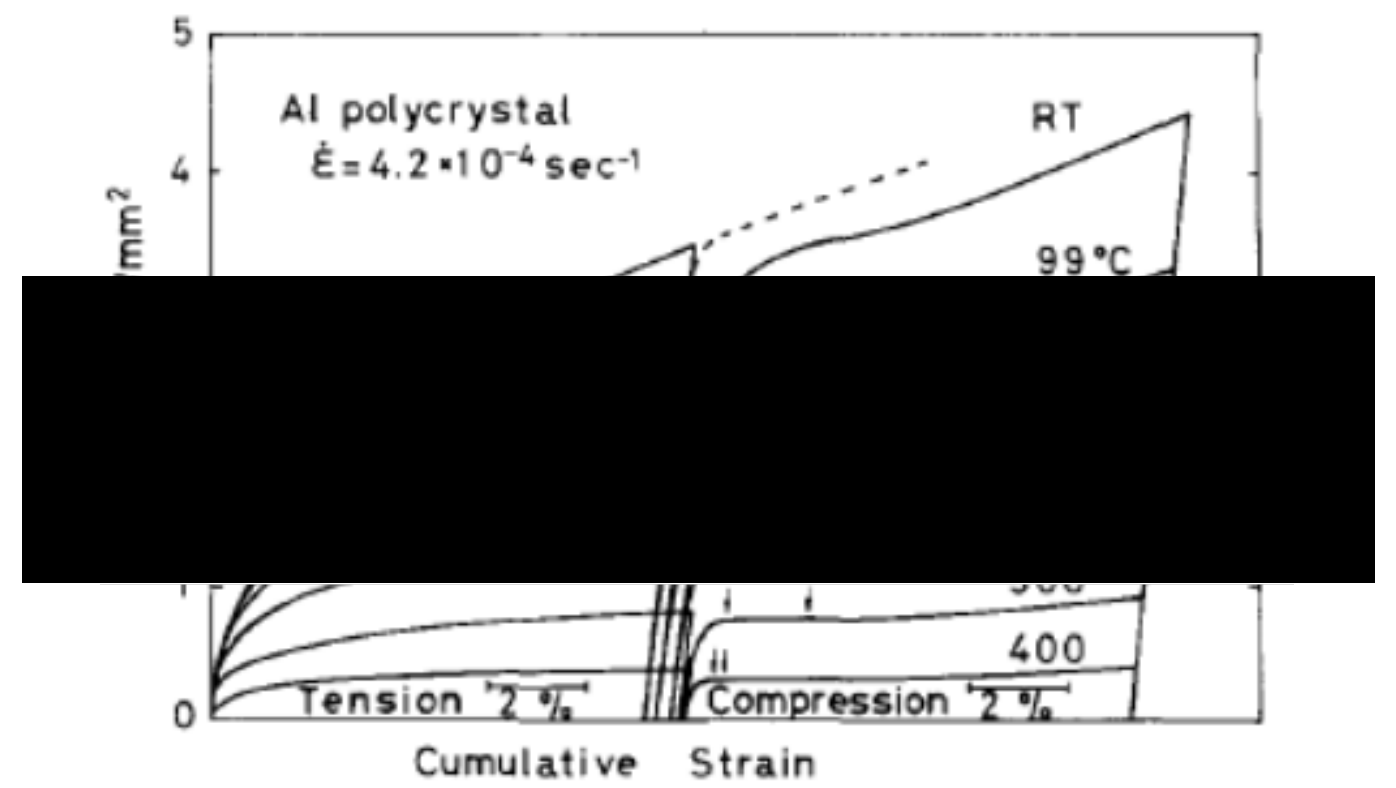

Figure 20-Forward-reverse (modulus) stress-strain curve and its dependence on temperature for cold formed strip steel

It can be seen above in Figure 20 that with an increase in test temperature, the forward-reverse stress-strain curve gradient, values for both pre and reverse strain are reduced significantly and the work-hardening rate in the reverse direction decreases to almost none as temperature increases. This increased temperature leads to annihilation of dislocations and therefore, a reduction of the dislocation density and reduced hardenability. In the example, by the time the deformation in the samples has reached that of the pre-strain (tension) phase, the compressive portion of the curve usually recovers to the same stress as the tension phase and in most cases surpasses it. However, for the room temperature sample this does not occur, the compressive section never recovers its properties after the initial pre-strain.

As discussed previously, the chemistry of the steel is closely linked with the pre-strain properties of the material and its work hardening characteristics. Carbon for example increases the work hardening of a material by pinning dislocations earlier. In the reverse direction, with an increase in carbon, the yield lowering effect and work hardening rate increases. This can be seen in Figure 21. 


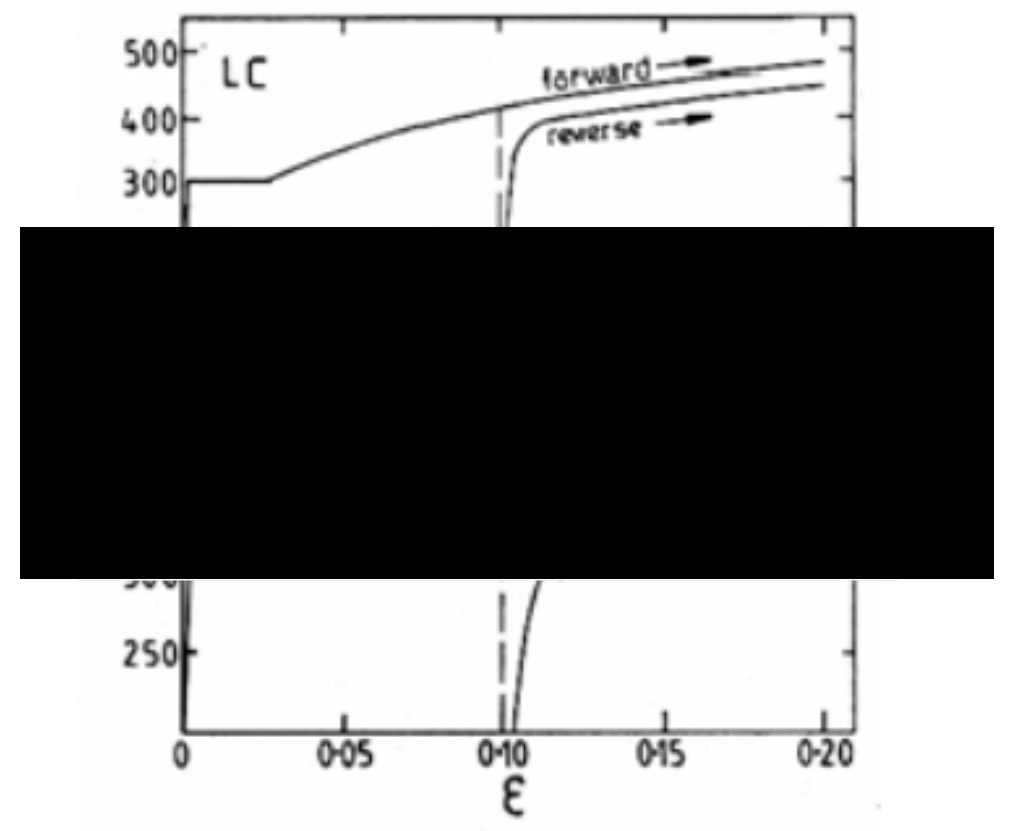

Figure 21-Typical stress-strain curve for high and low carbon steels showing the relative decrease in yield for the reverse deformation phase[47]

Alloying additions that strengthen the steel by either precipitation strengthening or solid solution strengthening tend to eliminate the stress increase plateau at the initiation of reverse deformation. The reason for this is that there is no dissolution of dislocation structures as there are more dislocation-obstacle interactions with features such as precipitates or solute atoms. [48][50] 


\subsection{Design of curved steel sections in situ}

There is no specific standard which covers the use of curved steel sections and the material properties are often assumed to be identical to the original, unbent section. However, resistance checks must still be performed with regards to the sections new geometric profile. The resistance checks presented in this section are based on the checks required in BS 5950-1, with appropriate modifications to account for the effects of curvature. Based on the research surrounding the Bauschinger effect, it is likely that an archway curved using cold-roller bending is more susceptible to out-of-plane buckling (shown in Figure 5-c[19], [47], [51], [52]) as it will have a reduced resistance to deformation when the loading is $180^{\circ}$ from that of the pre-strain. Though it is not specifically stated in the literature surrounding construction of steel structures, the curved section will likely have reduced resistance to out-of-plane buckling[3], [10], [51].

\subsubsection{Arches}

Members curved in elevation are often required to function, at least in part, as arches. Therefore, the bending moment in the curved member is less than would be experienced by a straight beam. However, the literature suggests that it is extremely important that the horizontal stiffness of the supports is high enough to counteract the lateral load exerted by the deflection of a curved beam when a vertical load is applied to it, which is likely greater when the section has been cold worked into shape using cold-roller bending[51]. If this is not performed adequately, the abutments will spread and the top of the arch will drop significantly, as shown in Figure 22.
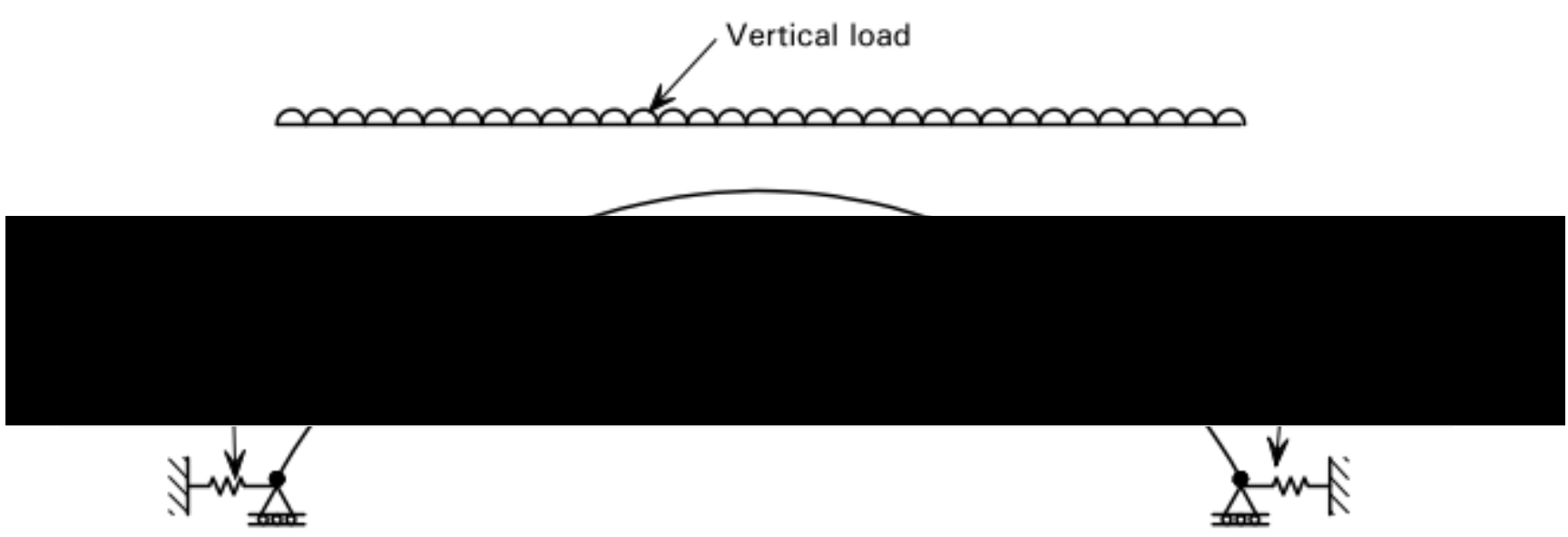

Figure 22-Curved member acting as an arch exerting a lateral load on abutments as a vertical load is applied upon it[2]

The consequence of this spread is that the axial compression and the bending moment in the arch must increase to resist the load, which might over-stress the arch member. The Bauschinger effect is known to reduce the resistance of a section $180^{\circ}$ from the original deflection. So it is likely that a 
section will have reduced capacity to resist this loading. The increasing deflection of the arch top will cause increasing axial compression, that the section is unable to support, leading to further spread of the abutments, causing the arch to collapse entirely.[2], [10], [47], [48], [53].

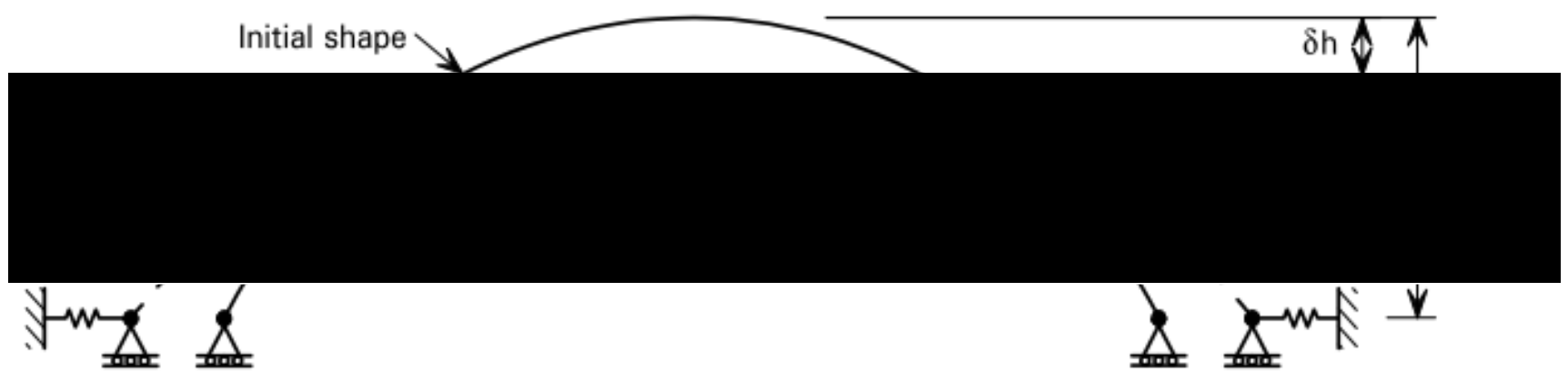

Figure 23-Deflected shape of a curved member exerting pressure on supporting abutments as vertical load is applied[2]

This effect is critical in arches with curvature, where there is a low rise compared with their span length. In the extreme, a flat arch with very low support stiffness behaves as a simply supported beam.

The reduction in the height of the arch can be compensated for or prevented in either of two ways. The first approach is to consider how the reduction in height must be limited to ensure that small deflection, first order frame analysis is appropriate.

First order analysis assumes that the stiffness of the structure remains constant and is not affected by changes in the frames geometry as it is loaded. This is a significant simplification of the problem. The principle of superposition applies to this approach. Where the analysis model remains the same, the results from analyses of different sets of applied actions can be added together and the results of individual design situations can be scaled. The analysis results are proportional to the applied actions. The second approach is to determine the deflection and axial compression resulting from the increasing spread of the abutments as the arch height reduces. This method requires secondorder analysis as the deflection of the beam is greater. [2], [21]. Second order analysis takes into account the change in stiffness brought about by changes to the geometry as loading is applied. Examples of this are cable structures, where a cable becomes apparently stiffer as it straightens out. 
The out of plane stresses and Bauschinger effect are often compensated for using the design yield strength, $p_{y d}$ the maximum allowable stress assumed the section can withstand before yielding. Though the design yield strength is not calculated taking Bauschinger effect into account, the large safety margin minimises its effect. For hollow sections, the guidance notes suggest using this reduced design strength for all resistance calculations. The maximum direct stresses normally occur at the corners of hollow sections. [2], [25] At these locations, the shear stresses arising from the shear force and torsion are near their maximum values; therefore the effect of the shear stresses must be taken into account by calculating a reduced design strength, $p_{y d}$

$$
p_{y d}=\left(p_{y}^{2}-3 \tau^{2}\right)^{0.5}
$$

\section{Equation 18-Design yield stress of a curved hollow section[2]}

Where: $\tau$ is the coexistent shear stress; the sum of the shear stresses from both the shear force and torsion. The shear stresses are calculated by dividing the shear forces by the shear area, the shear stresses due to torsion are calculated by dividing the torsion by the torsional constant, $\mathrm{C}$. The values input for these calculations are likely to change as cross sectional geometry is deformed during bending. 


\section{Materials}

Material selection is an important phase of the design process. Low strength steel is likely to be cheaper and are therefore often chosen preferentially due to strict budgets; however a larger crosssection will often be required to meet the requirements leading to increased weight of the final superstructure. A stronger steel grade, though more costly will lead to a reduced cross-section requirement. A smaller member cross-section leads to a reduced total steel requirement.

As mentioned in chapter 2 on page 22 there are a number of different forming methods for steel sections. The two section types investigated in this project are hot-finished and cold formed S355 grades, meaning that they both have a minimum yield strength of $355 \mathrm{MPa}$. However they have very different mechanical properties. Though both sections have the same specified minimum yield strength, cold-formed sections tend to be less ductile and therefore have a lower yield to UTS ratio than normalised, hot-finished steel. The cold-formed sections are also harder and have a lower impact resistance than the hot-finished sections. These differences are introduced by the different forming methods and chemistry of the grades. The cold-formed sections are heavily stressed during production leading to significant residual stresses and an elongated microstructure around the perimeter of the tube. The HAZ around the weld-line is clearly visible on inspection and has a fine acicular ferrite microstructure due to the rapid cooling after formation of the weld-line. The hotfinished sections have undergone a normalising process which removes the heat affected zone around the weld-line and any residual stresses introduced during the forming process. The heat treatment causes the steel to recrystallise, producing a finer, homogenous microstructure.

The sections examined in this project were produced by TATA steel in the UK and Holland. The Coldformed RHS was produced in the Netherlands and the hot-finished material was produced in the UK.

The suitability of curving these sections using cold-roller bending has been investigated

The chemistry of the steel is important as many of the alloying additions affect the materials strength, ductility and hardness along with the severity of the Bauschinger effect. 


\subsection{Chemical Analysis}

\begin{tabular}{|c|c|c|c|c|c|c|c|c|c|c|c|c|}
\hline $\begin{array}{l}\text { Section } \\
\text { Type }\end{array}$ & $\begin{array}{l}\text { C } \\
\text { (\%wt) }\end{array}$ & $\begin{array}{l}\text { Si } \\
\text { (\%wt) }\end{array}$ & $\begin{array}{l}\text { Mn } \\
\text { (\%wt) }\end{array}$ & $\begin{array}{l}P \\
\text { (\%wt) }\end{array}$ & $\begin{array}{l}S \\
\text { (\%wt) }\end{array}$ & $\begin{array}{l}\mathrm{Cr} \\
(\% w t)\end{array}$ & $\begin{array}{l}\text { Mo } \\
\text { (\%wt) }\end{array}$ & $\begin{array}{l}\mathrm{Ni} \\
(\% w t)\end{array}$ & $\begin{array}{l}\text { Al } \\
\text { (\%wt) }\end{array}$ & $\begin{array}{l}\mathrm{Ca} \\
\text { (\%wt) }\end{array}$ & $\begin{array}{l}\mathrm{Cu} \\
\text { (\%wt) }\end{array}$ & $\begin{array}{l}\text { Nb } \\
\text { (\%wt) }\end{array}$ \\
\hline $\begin{array}{l}\text { Hot } \\
\text { finished } \\
\text { RHS }\end{array}$ & 0.14 & 0.177 & 1.32 & 0.014 & 0.003 & 0.02 & $<0.01$ & 0.02 & 0.036 & 0.0004 & 0.019 & 0.028 \\
\hline $\begin{array}{l}\text { Cold } \\
\text { Formed } \\
\text { RHS }\end{array}$ & 0.17 & 0.013 & 1.4 & 0.016 & 0.004 & 0.04 & 0.01 & 0.03 & 0.044 & 0.0003 & 0.018 & 0.013 \\
\hline
\end{tabular}

Table 1-Chemical composition of the sample sections

It can be seen in Table 1 that the cold formed RHS has the higher carbon equivalent value (CEV) of the samples tes normally employed by TATA Steel UK. Both the forming method and higher CEV combined make this product sig material.

Both materials have carbon equivalent values well within the weldability limits for steel sections. With the hot-finishe cold-formed considered "good"[12], [54]

$$
C E V=\% C+\left(\frac{\% M n+\% S i}{6}\right)+\left(\frac{\% C r+\% M o+\% V}{5}\right)+\left(\frac{\% C u+\% N i}{15}\right)
$$

\section{Equation 19-Calculation of the Carbon Equivalent Value}




\section{Manipulation}

\section{Cold Bending}

The aim of this project was to assess the effect of cold-roller bending on the mechanical properties of hot-finished and cold-formed RHS with increasing strain rate. The RHS were curved using coldroller bending equipment with curvature being increased in stages from $0.25 \%$ to $5 \%$ strain $(0.25$, $0.5,1,2,3,4$ and $5 \%$ strain). The curved sections were then cut to $750 \mathrm{~mm}$ lengths. Each of these was then cut into three sections. Two, 300mm lengths were sent to Corby for tensile and Charpy testing; and one, $150 \mathrm{~mm}$ length was sent to Swansea University for microstructural analysis and Hardness testing. Cutting each length into three allowed comparative testing to be carried out on certain areas in tandem. For example, the intrados corner of each particular sample can be subjected to tensile, Charpy, hardness testing and microstructural analysis meaning that correlations between hardness and tensile strength for a particular condition can be analysed. Each sample was given its own unique sample code which allowed the results to be more easily compared.

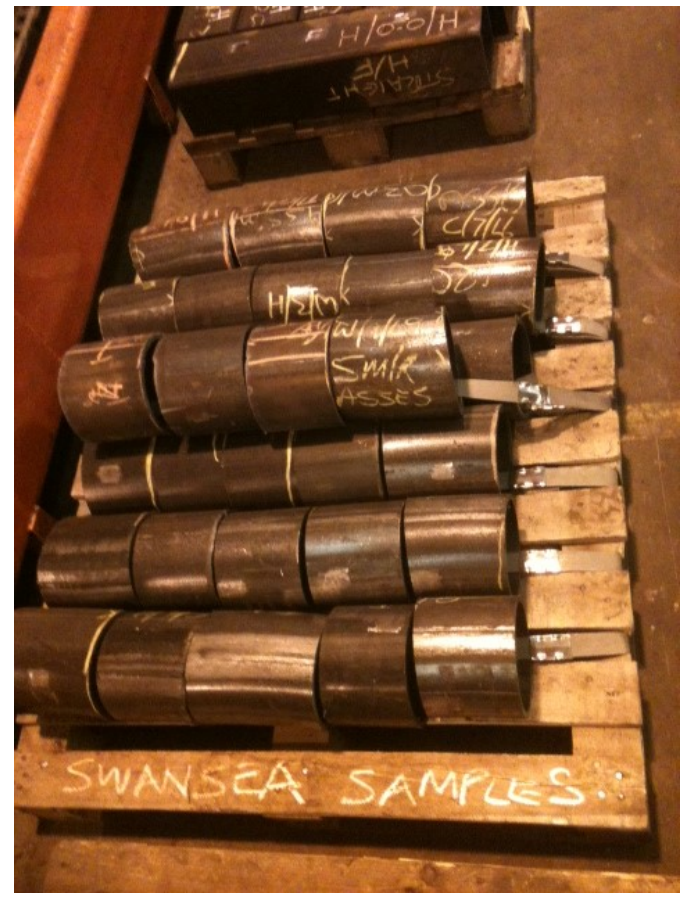

Figure 24-The image shows the circular tube samples shortly after being cut into short lengths for transportation to their testing site at Swansea 
Figure 25 indicates the locations from which the samples were taken from the RHS. These positions were chosen to ensure that all of the areas that were likely to be affected by the bending process were examined. The sides of the RHS and the neutral axis remain flat during bending. Due to the relative ease of testing the neutral axis, in industry all tensile testing is carried out with sections cut from this area. It is clear that this industry norm is not sufficient for examining the effect of the bending process on the mechanical properties. It is clear that the extreme fibres at the intrados and extrados undergo significantly more deformation than the side walls of the section, with the corners being most heavily affected. In cold-formed sections, these areas, will have already been heavily cold worked to produce the corner during the formation of the section profile. They were then deformed further during the curving procedure. For this project the testing has been carried out at all of the locations detailed in Figure 25 where possible to ensure that all of the likely areas of weakness are analysed. 

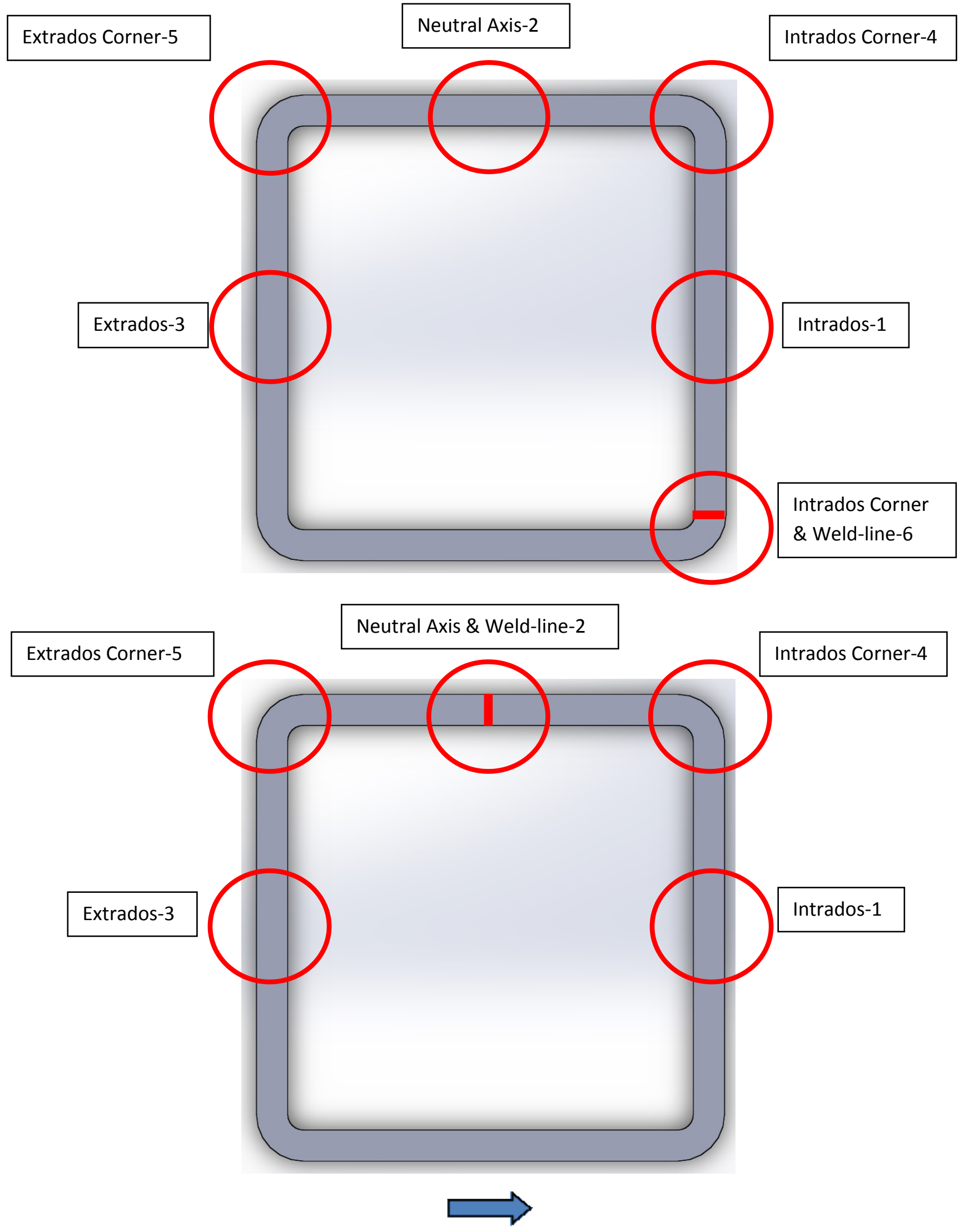

Figure 25-Schematic diagram showing the sampling position, number designation (1-6) and the bend direction (indicated with the blue arrow) of the rectangular hollow sections.

1-Intrados-inside surface of the bend, 2-Neutral axis of the bend and weld-line for the cold-formed samples

3-Extrados-outside surface of the bend, 4-Intrados corner, 5-Extrados corner

6-intrados corner containing a weld-line for the hot-finished samples 


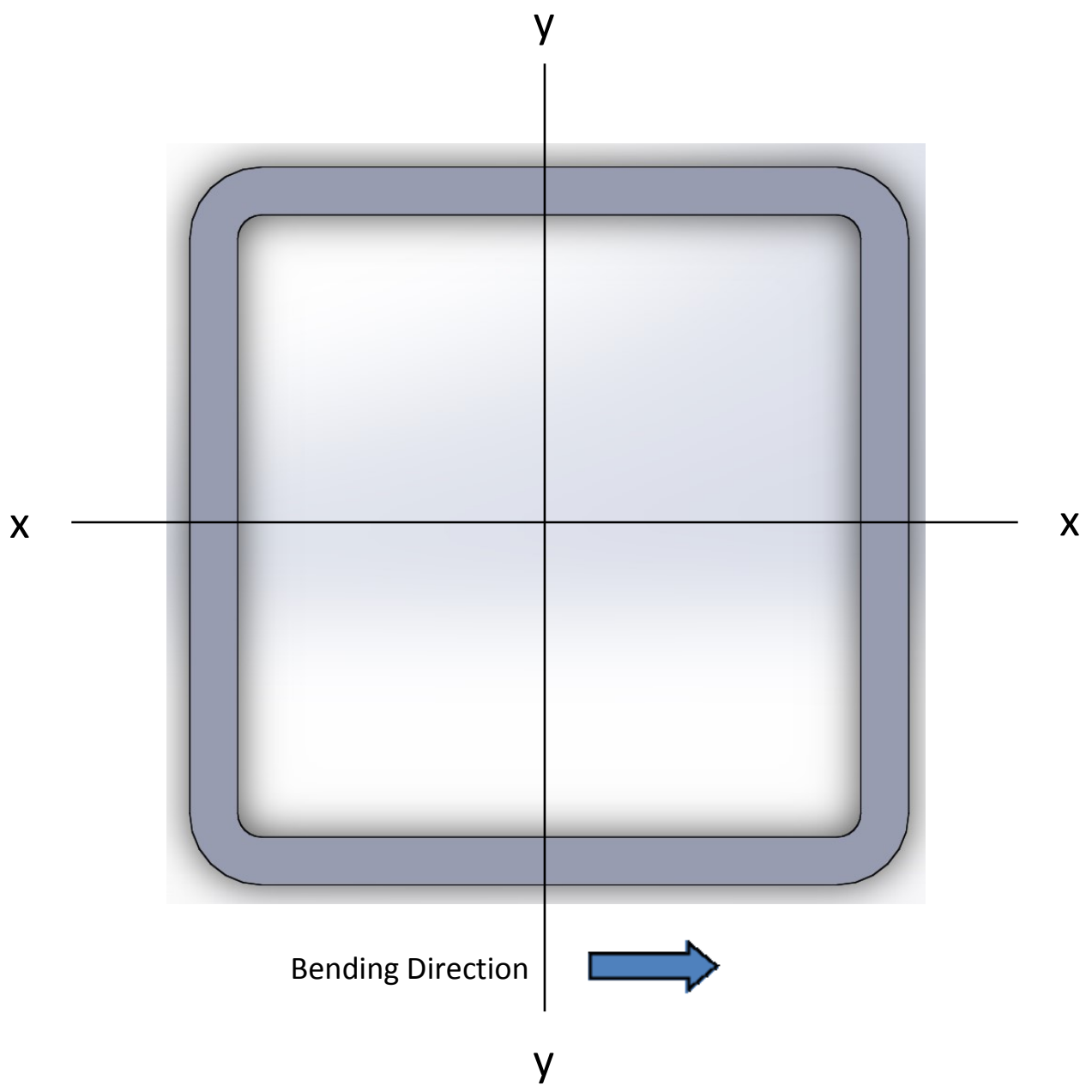

Figure 26-Schematic diagram indicating the bending direction and the denoted axis 


\section{Methodology}

\subsection{Microstructure}

The microstructure of the sections were examined to investigate any obvious signs of strain caused during the bending process. All areas detailed in Figure 25 were examined by removing small samples from the sample lengths using a precision saw. These samples were then mounted in Bakelite resin and labelled. Samples at this stage can be seen in Figure 27. The samples were then ground flat using silicon carbide paper pads. The samples were ground to an increasing surface quality until they were ready for polishing. The samples were then polished using Diamond suspension to a $1 \mu \mathrm{m}$ surface finish. Care must be taken when preparing a sample as bevelling or rounding of a sample can lead to poor image quality. When measurements are taken, it is important that the picture analysis software is fully calibrated to ensure correct results.

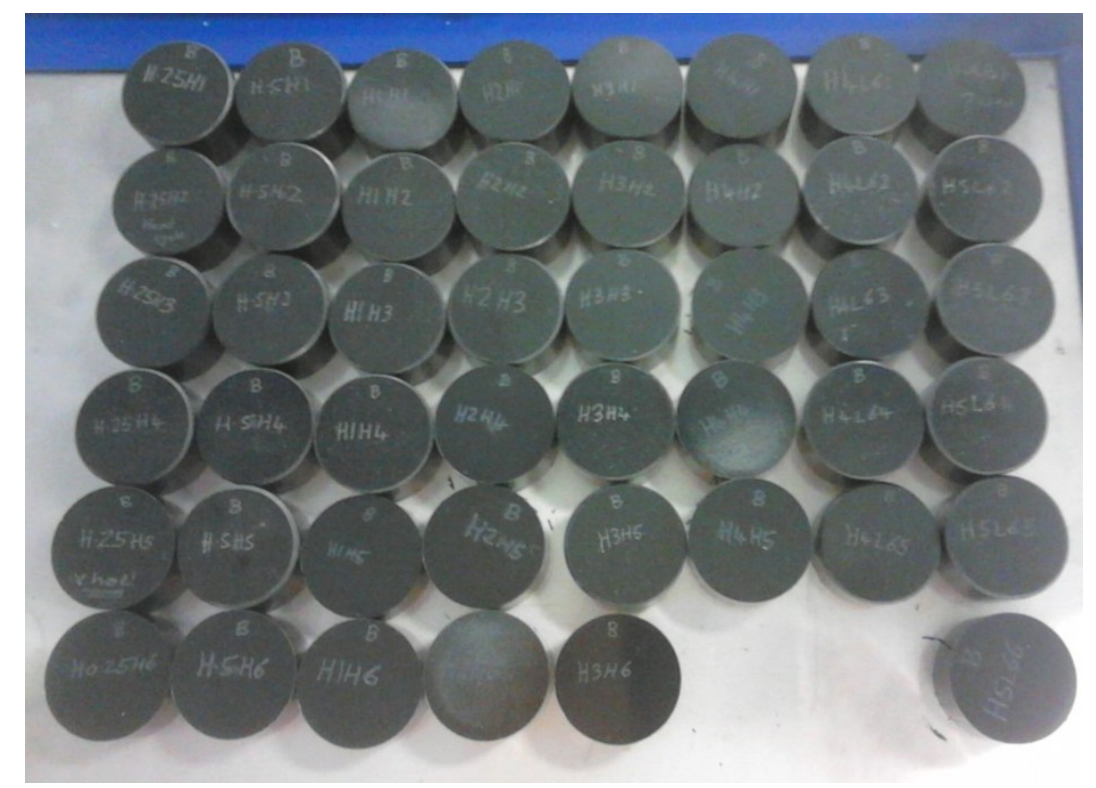

Figure 27-The image shows the hot formed tube samples that are to be used for microstructural analysis, mounted in resin.

The samples were then etched in $5 \%$ Nital solution. After etching the samples were rinsed with ethanol and dried to prevent over etching. The samples were then observed using a Nikon MA200 inverted metallographic microscope with analysis software.

Grain analysis software was calibrated before analysing the microstructure to ensure accurate measurements. 


\subsection{Hardness}

After microstructural analysis, the samples were sent to TATA Steel's Swinden Technology Centre (STC) in Rotherham for hardness testing. Automatic hardness testing equipment was used to assess both the inter and intra-sample hardness variations caused by the bending process.

The tests were specifically intended to indicate variation in:

- Through-thickness hardness of the sections; from bore edge to outer edge

- Hardness around the corner region perimeter; to specifically examine how wide the hardened region is though the thickness of the section

- Hardness across the weld-line and HAZ

The testing also allowed a comparison to be made between different areas on a particular sample as well as comparing the results from the same position on bends with a different strain rate.

The most commonly used hardness test is the Vickers hardness test[17] and was developed as an alternative to the Brinell method of measuring the hardness of materials. The Vickers test is often considered easier to perform than other hardness tests as the required calculations are independent of the size of the indenter. The Vickers test is the most versatile and can be used for all metals as it has one of the widest scales among hardness tests. The basic principle, as with all measures of hardness, is to determine a chosen material's ability to resist plastic deformation. The unit of hardness given by the test is known as the Vickers Hardness (HV). The hardness number is determined by the load over the surface area of the indentation. The HV number is then determined by the ratio $F / A$, where $F$ is the force applied to the diamond and $A$ is the surface area of the resulting indentation in square millimetres. A can be determined by the formula.

$$
H V=\frac{0.1891 F}{d^{2}}
$$

\section{Equation 20-Vickers Hardness}

Where: $\mathrm{d}=$ diagonal distance $(\mathrm{mm})$ and $\mathrm{F}=$ Force $(\mathrm{N})$ [17]

The hardness of the sample can be used to estimate the tensile strength of the section using conversion tables. This becomes especially useful when dealing with bent sections, as production of a tensile test sample requires it to be straightened, altering the properties slightly and giving results which aren't comparable to an unbent section. 
Surface hardness checks can be difficult due to a scale build-up on the surface. This scale would need to be removed before testing takes place. This renders dynamic hardness testing during production impractical. The curvature of the section can also alter the results.

Care must be taken when performing hardness testing as:

- Bevelling in a sample will produce a kite shaped indent, the same is also true for the rounded surface of a tube's corner during surface hardness testing.

- Equipment must be regularly calibrated in order to ensure correct loading, load time and measurement focus

- Indenting the sample will often cause a heave around the test point if this is measured due to poor maintenance of the equipment can lead to poor results.

The Vickers hardness of the samples was tested both at the surface as a non-destructive technique and through the cross section in order to establish what the effects of strain rate were on the hardness of the samples. 


\subsection{Tensile Testing}

Tensile testing is a fundamental method in which a sample is subjected controlled tension until failure. The results from the test are commonly used to select a material for an application, quality control, or to predict how a material will react under other types of forces. Properties that are directly measured during a tensile test are yield strength, ultimate tensile strength and maximum elongation. The reduction in area, caused by necking is recorded after the test is complete. From these measurements, a number of other properties of the material can be calculated including the: Young's Modulus, Poisson's ratio and the strain-hardening characteristics. The results are usually plotted in a graph of stress against strain. Uniaxial tensile testing is the most commonly used for obtaining the mechanical characteristics of isotropic materials. Due to the grain orientation caused during production of the tubes and subsequent bending, the tensile strength of the steel is not isotropic.

The machining of the samples was performed in the TATA Steel Corby workshop. The samples were cut from the tube sections using a cooled saw. The "dog-bone" shaped samples were then straightened so that they could be placed in the automatic tensile tester

The standard tensile testing was carried out in the TATA Steel Corby test house by trained technicians using an automated tensile tester produced by Schenck. The age of the machine meant that no stress strain plots could be produced, however, the yield strength, UTS and elongation of the sample is output by the equipment. Calibration of the machine is performed by UKAS accredited engineers when required.

The output of the tensile testing equipment was recorded in a table and the elongation of the sample is measured using a micrometre after failure.

\subsection{Tension-Compression Testing}

Tension-compression and compression-tension tests have been carried out on hot-finished material to determine the influence of the Bauschinger effect on the material when axial loading is reversed. A sample is first pulled beyond its elastic limit in tension to a set displacement. The load is then reversed and the section is compressed to a specified displacement. Other samples were first compressed beyond their elastic limit before pulling the section in tension to a specified displacement. The force required and the extension/compression of the sample is recorded by the equipment and the stress and strain experienced by the sample are calculated using the dimensions of the section. Alignment of the system is of particular importance for this method of testing as any misalignment can lead to early-onset buckling in the sample. Great care was taken to ensure that the sample was held orthogonal to the equipment in order to 
minimise the likelihood of buckling. The testing apparatus used was a Dartec $250 \mathrm{kN}$ which is based at the Swansea University Bay Campus. Using specially designed threaded collets to allow for both tension and compression of the same sample. 


\subsection{Three-point Bend Testing}

Three-point bend tests have been performed on hot-finished and cold-formed material to determine the influence of the Bauschinger effect on the material when flexural loading is reversed. Threepoint bend testing is a simple method to determine a material's resistance to bending. The testing method requires a tensile/compression tester and a specialised jig in order to perform the test. The test piece was supported from below on 2 support rollers and the load was applied at the centrepoint between the supports from above. The sample was then bent to a specified displacement. The sample was then turned over after the load was removed and re-straightened by bending the sample back to a straight piece. This was performed to assess any reduction is proof stresses after initial bending. The sample was then turned over a final time and re-bent to a displacement equal to the original bend. The force and displacement is recorded by the testing machine and the flexural stress and strain are calculated from the values and the dimensions of the section. The testing apparatus used was a Dartec $250 \mathrm{kN}$ which is based at the Swansea University Bay Campus. 


\subsection{Impact Testing}

There are two main types of impact test commonly used for steel samples. The most common of these is the Charpy impact test. Both methods include impacting a notched sample with a swinging mass. The Charpy Impact test was used in this project and is a standardised test which determines the amount of energy absorbed by a material during instantaneous fracture. This absorbed energy is a measure of a given material's toughness and acts as a tool to study temperature-dependent ductile-brittle transition. It is widely applied in industry, since it is easy to prepare and conduct and results can be obtained quickly and cheaply. However, machining test samples can be time consuming. The impact resistance of the sections bent during this project have been compared to assess toughness and to determine which bending conditions affect the final product least.[17] The impact testing was carried out in the test house at TATA Steel Corby on an Instron machine.

\subsection{Dimensional Checks}

\subsubsection{Wall Thickness}

During bending, the area on the inside of the bend is in compression, the area on the outside of the bend is in tension and, usually equidistant between the two is the neutral axis where no net force is applied. Due to these applied forces, the lengths of the surfaces inside and outside the bend are altered. The Intrados is compressed and shortened and the extrados is pulled and extended. This leads to a reduction in wall thickness at the outside of the bend. Conversely, the compression experienced at the bend interior will lead to a considerable increase in wall thickness or buckling. Poisson's ratio is the ratio of lateral strain to axial strain in a specimen that is loaded axially. The physical wall thickness can be measured in a number of ways with varying degrees of accuracy. A Coordinate measuring machine (CMM) probe can be used to precisely measure the wall thickness and can then be programmed to take measurements automatically; eliminating human error. However, for this method to work both correctly and accurately the tube needs to be cut into sections. This is not ideal for the company bending the section. There would be a lot of downtime while the wall thicknesses are measured in order to ensure the sections are within specification. An alternative method is ultrasonic testing. Ultrasonic testing can be used to measure the wall thickness of the section without any need for the tube to be cut up so there is no sacrificial tube required for wall thickness checks and the data is fed back to the operators more quickly allowing for changes which leads to improved wall thicknesses in future bends. The percentage change in wall thickness can be calculated by using the following equation:

$$
\text { Wall Thickness }=\frac{\mathrm{T}-\mathrm{t}}{\mathrm{t}} \times 100
$$




\section{Equation 21-Percentage Change in Wall Thickness}

Where: $\mathrm{T}=$ Current Wall Thickness $(\mathrm{mm})$ and $\mathrm{t}=$ Original Wall Thickness $(\mathrm{mm})$

These changes in wall thickness can lead to differing mechanical properties along the length of the tube.

In this project the wall thickness of the tubes are measured using Vernier callipers as the tubes are already cut up, making this task easier. The wall thickness is measured in a number of places and an average for that area is calculated and tabulated.

\subsubsection{Out of Square}

During bending the cross section of the tube is altered. In general, when a tube is bent, the X-X length is reduced and the $Y-Y$ length is increased, for example in Figure 26. This change in shape will give the tube different properties when forces are applied in differing directions. Therefore, the out of square, or how rectangular the section becomes after bending, must be carefully monitored. The outer dimensions of the section are measured using a set of external callipers. It is a quick check taken in both the $X-X$ and $Y-Y$ directions at multiple locations along the tube length. Out of square is the ratio of depth to breadth. The larger the difference between the two values the larger the percentage will be. A square will have an out of square value of $0 \%$ as both values are equal.

The percentage out of square is calculated by inputting the depth and breadth into the following equation:

$$
\text { Out of Square }=\frac{2 \times(D-B)}{(D+B)} \times 100
$$

\section{Equation 22-Out of Square}

Where: $\mathrm{D}=$ Depth of the section and $\mathrm{B}=$ Bredth of the section

\subsubsection{Corner Radii}

The corner radii has been measured using internal and external radius gauges and recorded. 


\subsection{Geometric Properties}

The measured dimensions of the sections were used to calculate the geometric properties. The geometric properties of a section are used to determine a section's suitability for use in a structure during the design process. The geometric properties of the sections have been calculated in order to assess the effect of the roller-bending process on the properties. The geometric property equations are stated in the respective standards for the hot-finished and cold-formed material[7], [9], [55], [56]. The geometric properties that were calculated for comparison were:

- Mass per unit length-

- The mass of the section per unit length is used to calculate the weight of a section in-situ which is needed to design supporting structures.

- Cross sectional area

- The cross sectional area is needed to determine the safe working load based on its yield strength and UTS. It is particularly important for tension calculations.

- Second moment of area

- The second moment of area is a measure of a shapes resistance to bending caused by loading in a particular direction.

- Radius of gyration

- The radius of gyration is used to estimate the stiffness of a column in compression and the subsequent likelihood of bucking.

- Section Modulus

- There are two types of section modulus, elastic and plastic.

- Elastic section modulus is used to determine the yield moment

- Torsional inertia constant

- Torsional modulus constant

- These constants are used to determine a sections resistance to torsion.

- Shear modulus

- Shear modulus is used to determine a sections resistance to shear stress. 
All of the above geometric information is needed for correct engineering design of a structure. However, a number of effects of the bending process such as concavity of the walls of a RHS are not included in this list as they are assumed to lie within steel section specifications. Currently there is no construction standard for curved hollow sections; however, this is likely to change in the near future. All of the sections tested in this investigation have been compared against two standards; the production standard for straight sections (BS EN 10210:2006 and 10219:2006 for hot and cold formed sections respectively[7], [9], [55], [56]) and the construction standard for steel products (BS EN 1090:2009[4]). These comparisons will demonstrate the alterations in properties of the curved sections when compared to those of the straight sections and to assess how well they will perform in-situ.

The second moment of area is important for use in construction calculations as it is property of shape which aids the prediction of deflection, bending and stress under loading. It has been calculated in both the $X-X$ and $Y-Y$ directions using the mean wall thickness as well as the mean height and width dimensions.

\subsubsection{Mass per unit length, $M$}

$$
M=\mathbf{0 . 7 8 5 A}
$$

\section{Equation 23-Mass per unit length of RHS}

This equation is based on the average density of steel at room temperature.

\subsubsection{Cross sectional area, $A$}

$$
A=\frac{2 T(B+H-2 T)-(4-\pi)\left(r_{0}^{2}-r_{i}^{2}\right)}{10^{2}}
$$

\section{Equation 24-Cross-sectional area of a RHS}




\subsubsection{Second Moment of Area, I}

$$
I_{y y}=\frac{1}{10^{4}}\left[\frac{B H^{3}}{12}-\frac{(B-2 T)(H-2 T)^{3}}{12}-4\left(I_{g}+A_{g} h_{g}^{2}\right)+4\left(I_{\xi \xi}+A_{\xi} h_{\xi}^{2}\right)\right]
$$

Equation 25-Second moment of area in the Y-Y (vertical) direction of a RHS

$$
I_{x x}=\frac{1}{10^{4}}\left[\frac{H B^{3}}{12}-\frac{(H-2 T)(B-2 T)^{3}}{12}-4\left(I_{g}+A_{g} h_{g}^{2}\right)+4\left(I_{\xi \xi}+A_{\xi} h_{\xi}^{2}\right)\right]
$$

Equation 26-Second moment of area in the X-X (horizontal) direction of a RHS

Where

$$
A_{g}=\left(1-\frac{\pi}{4}\right) r_{o}^{2}
$$

Equation 27-Ag (outside corner radius)

$$
A_{\xi}=\left(1-\frac{\pi}{4}\right) r_{i}^{2}
$$

Equation $28-A_{\xi}$ (inside corner radius)

$$
h_{g y y}=\frac{H}{2}-\left(\frac{10-3 \pi}{12-3 \pi}\right) r_{o}
$$

Equation 29- $h_{\text {gyy }}$ (outside corner radius - vertical)

$$
h_{g x x}=\frac{B}{2}-\left(\frac{10-3 \pi}{12-3 \pi}\right) r_{o}
$$

Equation 30- $h_{g x x}$ (outside corner radius - horizontal)

$$
h_{\xi y y}=\frac{H-2 T}{2}-\left(\frac{10-3 \pi}{12-3 \pi}\right) r_{i}
$$

Equation 31- $h_{g y y}($ inside corner radius - vertical)

$$
h_{\xi x x}=\frac{B-2 T}{2}-\left(\frac{10-3 \pi}{12-3 \pi}\right) r_{i}
$$

Equation $32-h_{\mathrm{gxx}}$ (inside corner radius-horizontal) 


$$
I_{g y y}=\left(\frac{1}{3}-\frac{\pi}{16}-\frac{1}{3(12-3 \pi)}\right) r_{o}^{4}
$$

Equation 33

$$
I_{\xi y y}=\left(\frac{1}{3}-\frac{\pi}{16}-\frac{1}{3(12-3 \pi)}\right) r_{i}^{4}
$$

Equation 34

5.8.4. Radius of Gyration

$$
i_{y y}=\sqrt{\frac{I_{y y}}{A}}
$$

Equation 35-Radius of gyration in the Y-Y (vertical) direction of a RHS

$$
i_{x x}=\sqrt{\frac{I_{x x}}{A}}
$$

Equation 36-Radius of gyration in the X-X (horizontal) direction of a RHS

5.8.5. Elastic section Modulus

$$
W_{\text {el } y y}=\frac{2 I_{y y}}{H} \times 10
$$

Equation 37-Elastic section modulus in the Y-Y (vertical) direction of a RHS

$$
W_{e l x x}=\frac{2 I_{y y}}{B} \times 10
$$

Equation 38-Elastic section modulus in the X-X (horizontal) direction of a RHS

\subsubsection{Plastic section modulus}

$$
W_{p l y y}=\frac{1}{10^{3}}\left[\frac{B H^{2}}{4}-\frac{(B-2 T)(H-2 T)^{2}}{4}-4\left(A_{g} h_{g}\right)+4\left(A_{\xi} h_{\xi}\right)\right]
$$

Equation 39-Plastic section modulus in the Y-Y (vertical) direction of a RHS

$$
W_{p l x x}=\frac{1}{10^{3}}\left[\frac{H B^{2}}{4}-\frac{(H-2 T)(B-2 T)^{2}}{4}-4\left(A_{g} h_{g}\right)+4\left(A_{\xi} h_{\xi}\right)\right]
$$

Equation 40-Plastic section modulus in the X-X (horizontal) direction of a RHS 
5.8.7. Torsional Inertia Constant

$$
I_{t}=\frac{1}{10^{4}}\left[T^{3} \frac{h}{3}+2 K A_{h}\right]
$$

Equation 41-Torsional inertia constant

Where

$$
K=\frac{2 A_{h} T}{h}
$$

Equation 42-K-strength coefficient

Where

$$
h=2[(B-T)+(H-T)]-2 R_{C}(4-\pi)
$$

Equation 43-effective height

$$
A_{h}=(B-T)(H-T)-R_{c}^{2}(4-\pi)
$$

Equation 44- $A_{h}$

Where

$$
R_{c}=\frac{r_{o}+r_{i}}{2}
$$

Equation $45-\mathbf{R}_{\mathrm{c}}$

5.8.8. Torsional Modulus Constant

$$
C_{t}=10\left[\frac{I_{t}}{T+K / T}\right]
$$




\section{Results and Analysis}

Curving steel sections, either by hot or cold forming is known to alter the mechanical properties and often the dimensions of the section. For example, the hardness of a tube is likely to change dramatically after forming.[16] A number of testing methods are available for assessing the significance of these changes in properties. Some tests are destructive and require samples to be removed from the section, such as Charpy impact testing; cross sectional hardness measurements and microstructure analysis. Other testing is non-destructive and can be performed without any major damage to the section, such as external, dynamic hardness testing, out of square measurements and ultra-sonic wall thickness measurements. The methods used and the results collected have been detailed in this section. 


\subsection{Microstructural Analysis}

\subsubsection{Grain Size Analysis}

The grain sizes of the rectangular hollow sections were measured using the circular intercept method. The results stated are the ASTM grain sizes measured at sample positions 1-5/6 over the cross section for both the hot-finished and cold-formed samples. The measurements were taken from the mid-gauge point of each sample area.

\subsubsection{Hot-finished Rectangular Hollow Section Grain Size}

\begin{tabular}{|llllllll|}
\hline Sample ID & 1 & 2 & 3 & 4 & 5 & 6 & MEAN \\
\hline $\mathbf{H} / \mathbf{0 . 2 5 / H}$ & 11.4 & 12.2 & 11.5 & 11.7 & 11.4 & 11.5 & 11.6 \\
\hline $\mathbf{H} / \mathbf{0 . 5 0 / H}$ & 11.4 & 11.6 & 11.9 & 10.9 & 11.4 & 11.8 & 11.5 \\
\hline $\mathbf{H} / \mathbf{1 . 0 0 / H}$ & 12.2 & 12 & 11.8 & 12 & 12.1 & 11.4 & 11.9 \\
\hline $\mathbf{H / 2 . 0 0 / H}$ & 12.2 & 11.7 & 12.2 & 11.7 & 11.6 & 12 & 11.9 \\
\hline $\mathbf{H / 3 . 0 0 / H}$ & 11.8 & 11.8 & 11.9 & 11.9 & 11.3 & 12 & 11.8 \\
\hline $\mathbf{H / 4 . 0 0 H}$ & 11.6 & 11.9 & 11.8 & 11.9 & 11.7 & & 11.8 \\
\hline $\mathbf{H / 4 . 0 0 / L 6}$ & 11.9 & 11.9 & 11.7 & 11.8 & 11.4 & & 11.7 \\
\hline $\mathbf{H / 5 . 0 0 / L 6}$ & 11.5 & 11.8 & 11.5 & 10.6 & 11.5 & 11.4 & 11.4 \\
\hline
\end{tabular}

Table 2-Mean ASTM grain size of the hot finished RHS samples

\subsubsection{Cold-formed Rectangular Hollow Section Grain Size}

\begin{tabular}{|lllllllll|}
\hline Sample ID & 1 & 2 & 3 & 4 & 5 & 6 & MEAN \\
\hline C/0.25/H & 11.4 & 11.1 & 11.6 & 11.4 & 11.8 & N/A & 11.5 \\
\hline C/0.50/H & 11.2 & & 11.7 & 11.9 & 10.3 & N/A & 11.3 \\
\hline C/1.00/H & 10.9 & 11.4 & 11.2 & 11.1 & 11.4 & N/A & 11.2 \\
\hline C/2.00/H & 11.7 & 11.5 & 11.9 & 11.2 & 11 & N/A & 11.5 \\
\hline C/3.00/H & 11.9 & 11.4 & 11.5 & 11.7 & 11.1 & N/A & 11.5 \\
\hline C/4.00/H & 11.9 & 11.4 & 11.5 & 11.7 & 11.1 & N/A & 11.5 \\
\hline C/4.00/L4 & 11.4 & 11.7 & 11.9 & 12.1 & 11.7 & N/A & 11.8 \\
\hline C/5.00/L3 & 10.8 & 12.6 & 11.7 & 11.5 & 11.9 & N/A & 11.7 \\
\hline
\end{tabular}

Table 3 Mean ASTM grain size of the cold formed RHS samples 
There was no significant difference in the measured grain sizes of any of the samples. No clear conclusions can be drawn from these tables alone. All of the sections had a fine grain size consistent with aluminium treated steel which has been produced using fine grain practices. To better understand the performance of the sections, it was also necessary to consider the shape of the grains (equiaxed or elongated) and their orientation within the bulk material. This has been achieved using an optical microscope to observe the cross section. 


\subsubsection{Phase Determination}

\subsubsection{Hot-finished Square Hollow Section}

\section{Low Strain Body}

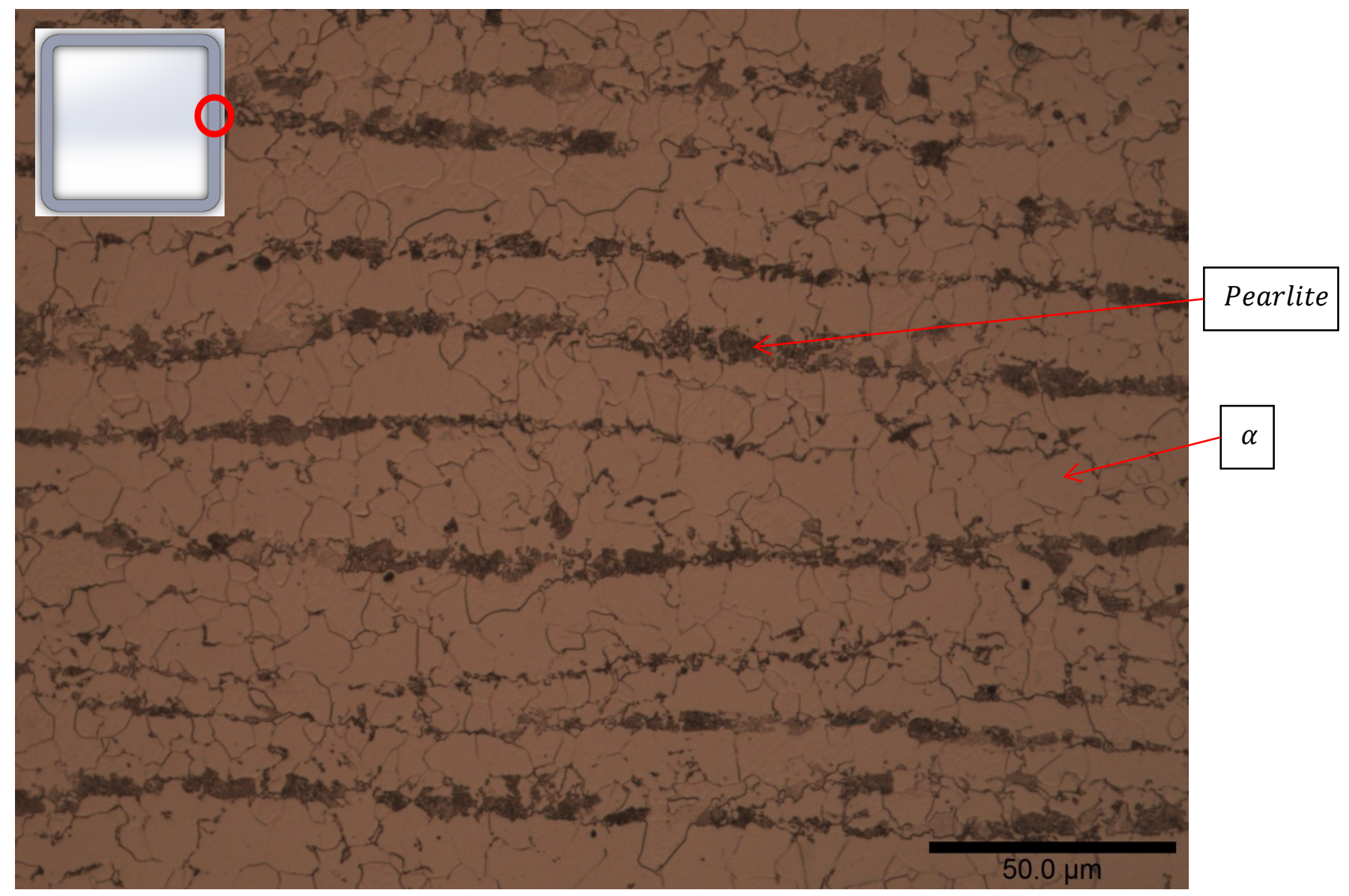

Figure 28-Hot-finished sample bent to $0.25 \%$ strain intrados flange, magnification $500 x-\mathrm{H} 0.25 \mathrm{H} 1$

Figure 28 shows the low strain, body microstructure of the TATA Steel hot finished RHS at 500x magnification. It has a fine grain ferrite microstructure with Pearlite banding throughout. The microstructure shows banding of Pealite and other alloying additions that have not dissolved into the ferrite during the initial forming process. There is no evidence of elongated grains as a result of the cold working caused by the roller bending process. 


\section{Low Strain Corner}

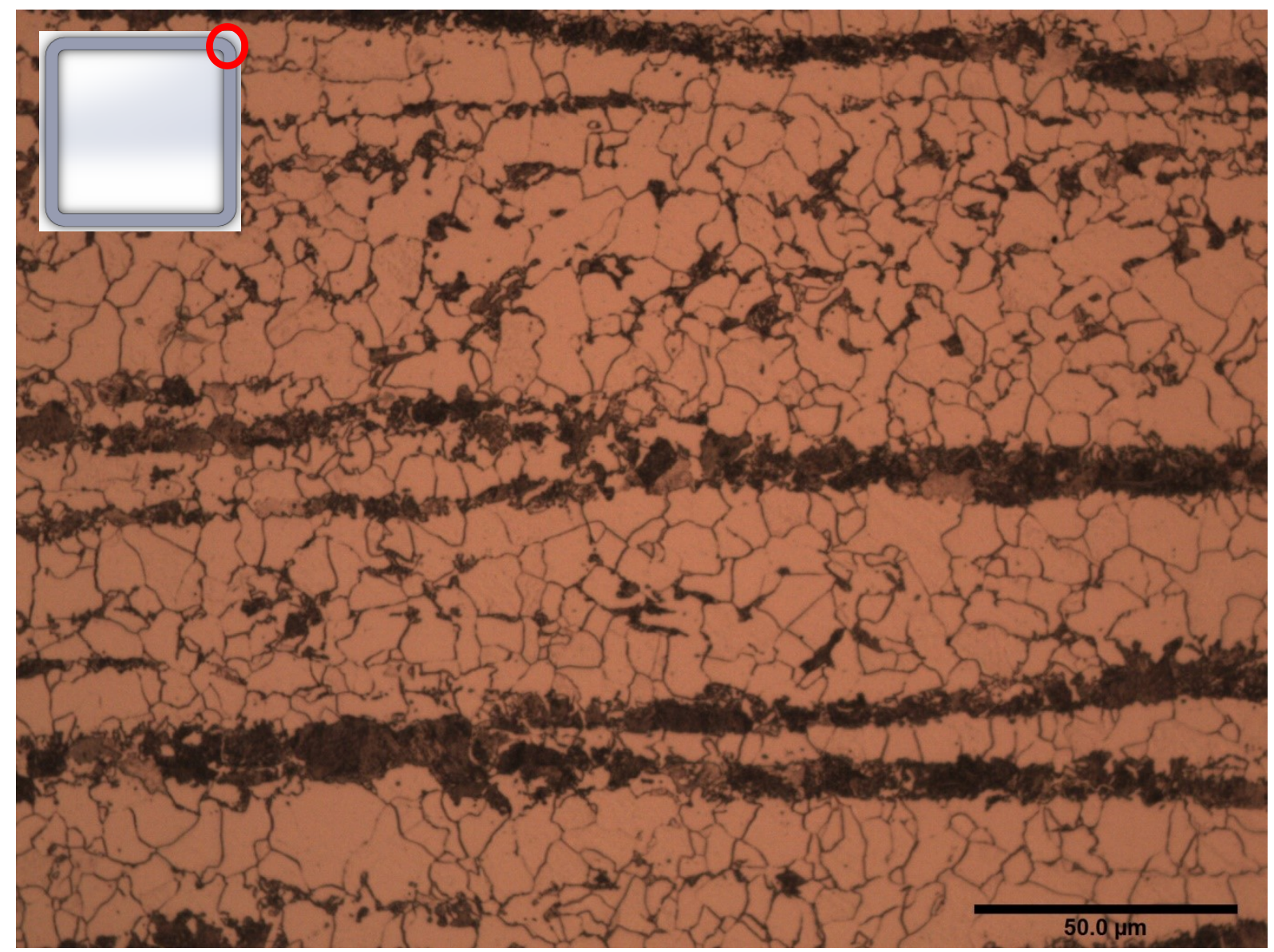

Figure 29 -Hot-finished sample bent to $0.25 \%$ strain, intrados corner, magnification $500 x-\mathrm{H} 0.25 \mathrm{H} 4$

Figure 29 shows the low strain, intrados corner microstructure of the TATA Steel hot finished RHS at 500x magnification. There is no discernible difference in microstructure between the corner and the flat side of the section shown in Figure 28. The corner would therefore be expected to have comparable properties to the rest of the tube. Again, there is no evidence of the effect of cold working through the roller bending process. 


\section{Low Strain Weld-line}

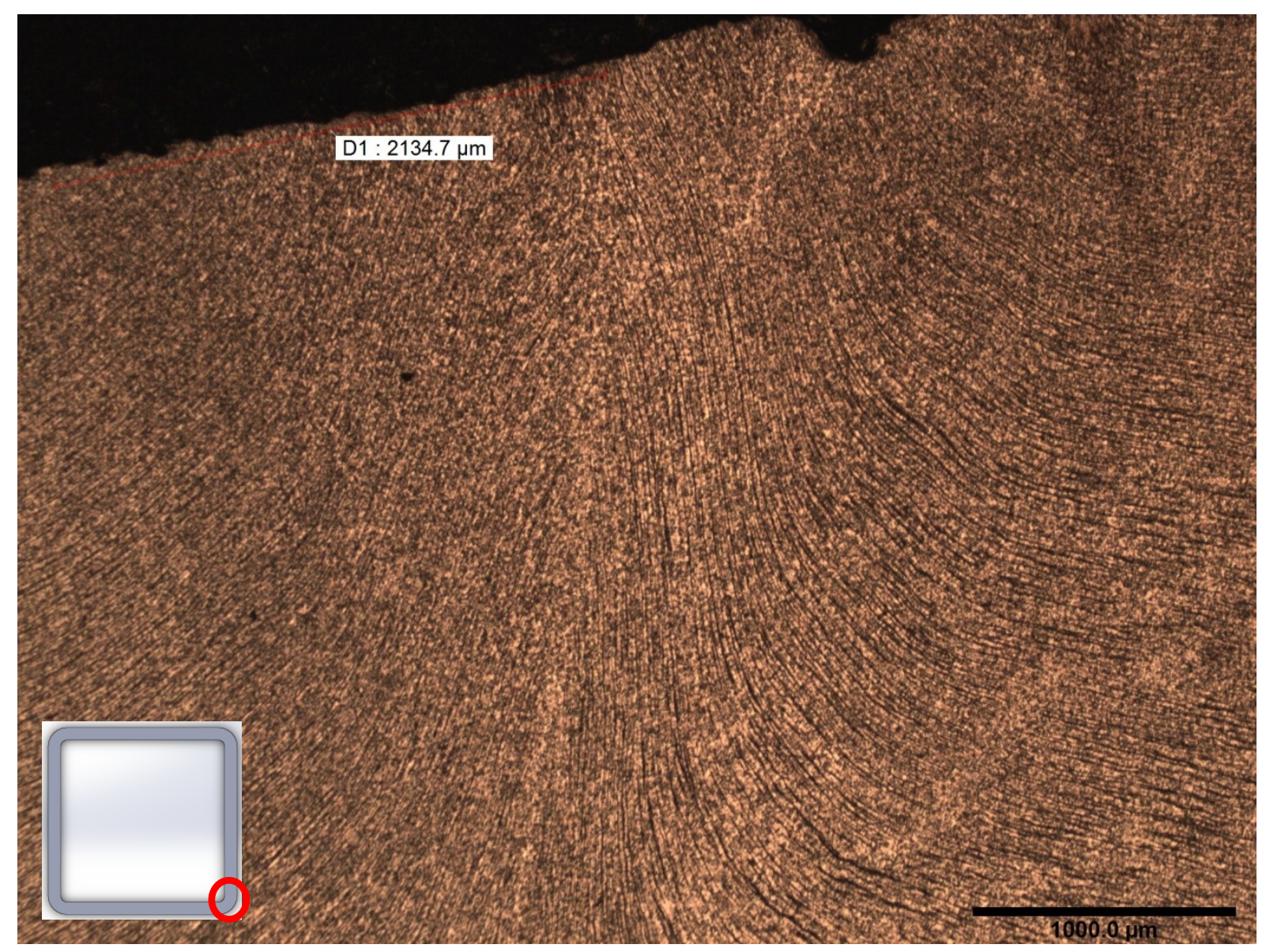

Figure 30 -Hot-finished sample bent to $0.25 \%$ strain, intrados corner weld-line, magnification $25 x$ $\mathrm{H} 0.25 \mathrm{H} 6$

Figure 30 shows the low strain, weld-line microstructure of a TATA Steel hot finished RHS at $25 \mathrm{x}$ magnification. Deviation of the bending adjacent to the weld-line can be seen clearly. This is caused during the high frequency welding operation when the molten strip edges are pressed together. This microstructure remains in place, even after the normalising process. At the widest point, the HAZ is approximately $2 \mathrm{~mm}$ either side of the weld-line. 


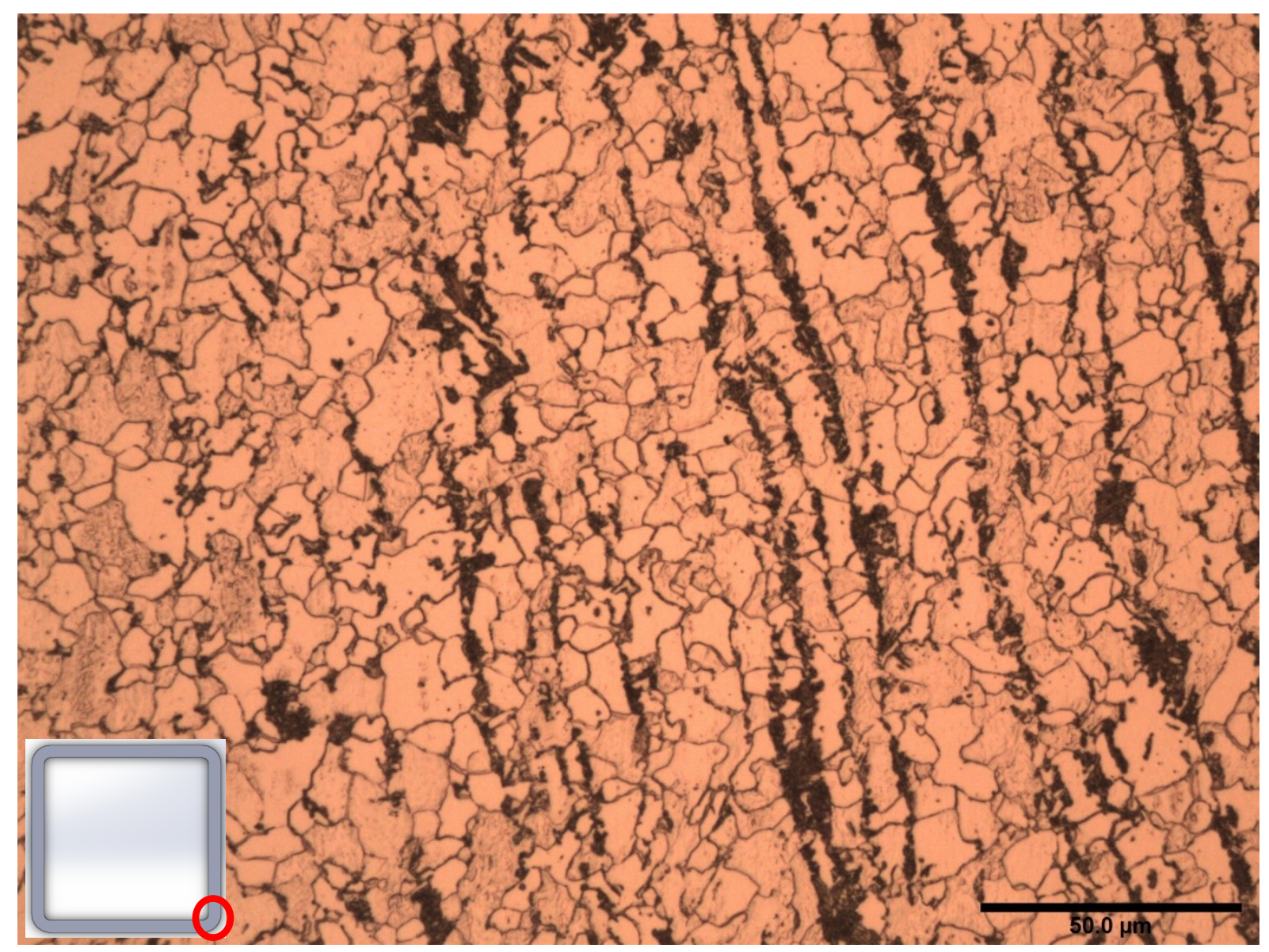

Figure 31-Hot-finished sample bent to $0.25 \%$ strain, intrados corner weld-line, magnification $500 x-$ H0.25H6

Figure 31 shows the low strain, weld-line microstructure of a TATA Steel hot finished RHS at 500x magnification. There is no discernible difference in grain size or shape between the weld and the flat sides of the section although, as shown in Figure 30, the banding in now in a different orientation across the sample. This similar microstructure would suggest that the weld-line would be expected to have comparable properties to the rest of the section. Again, there is no evidence of any effect of cold working as a result of the roller bending process. 


\section{Low Strain Pushout}

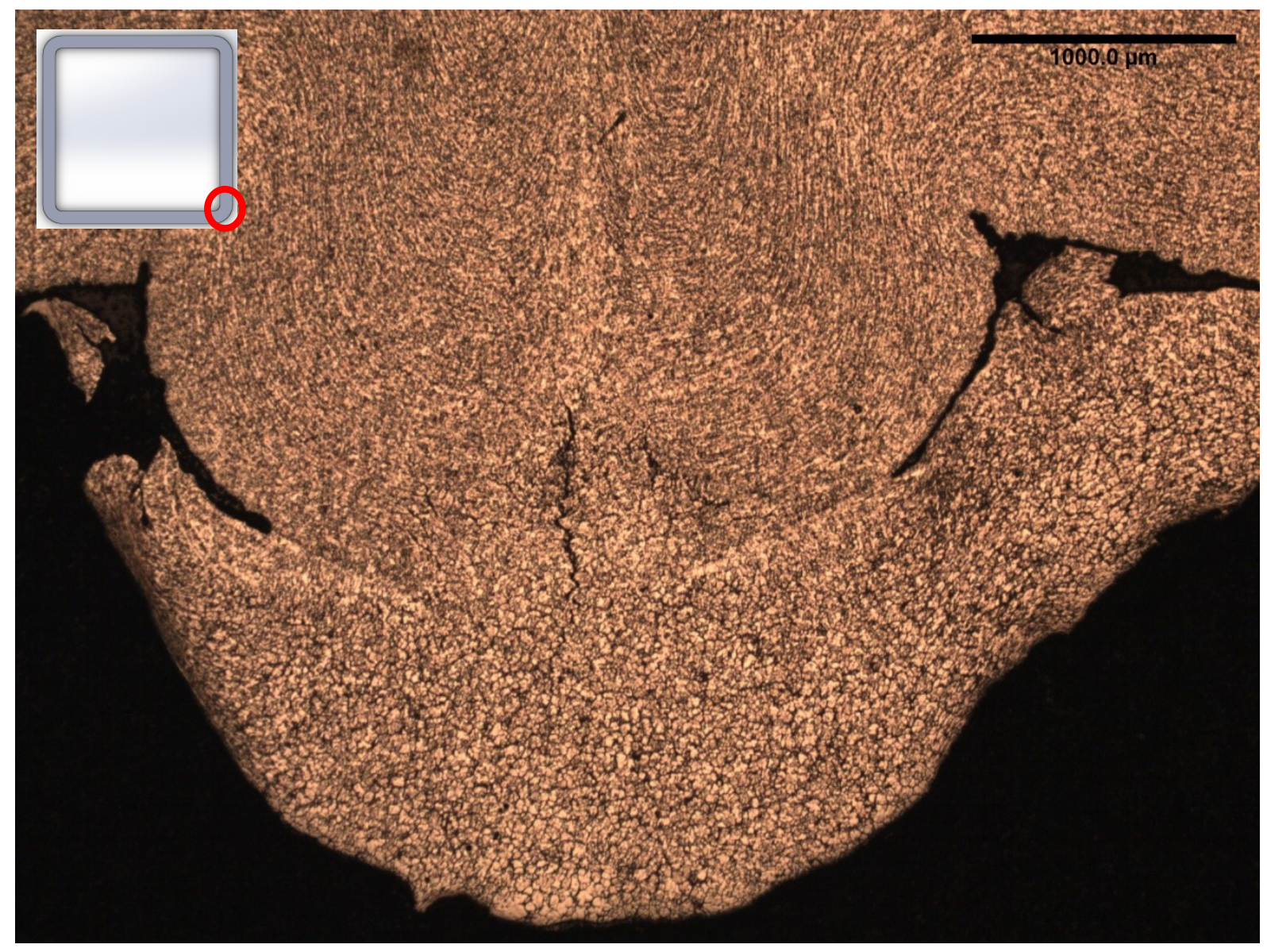

Figure 32-Hot-finished sample bent to $0.25 \%$ strain, intrados corner weld-line pushout, magnification 25x $\mathrm{H} 0.25 \mathrm{H} 6$

Figure 32 shows the low strain, weld-line pushout microstructure of the TATA Steel hot finished RHS at $25 \mathrm{x}$ magnification. The deviation in the banding adjacent the weld-line can be seen clearly. A collection of inclusions can be seen on the weld-line at the centre of the feature. 


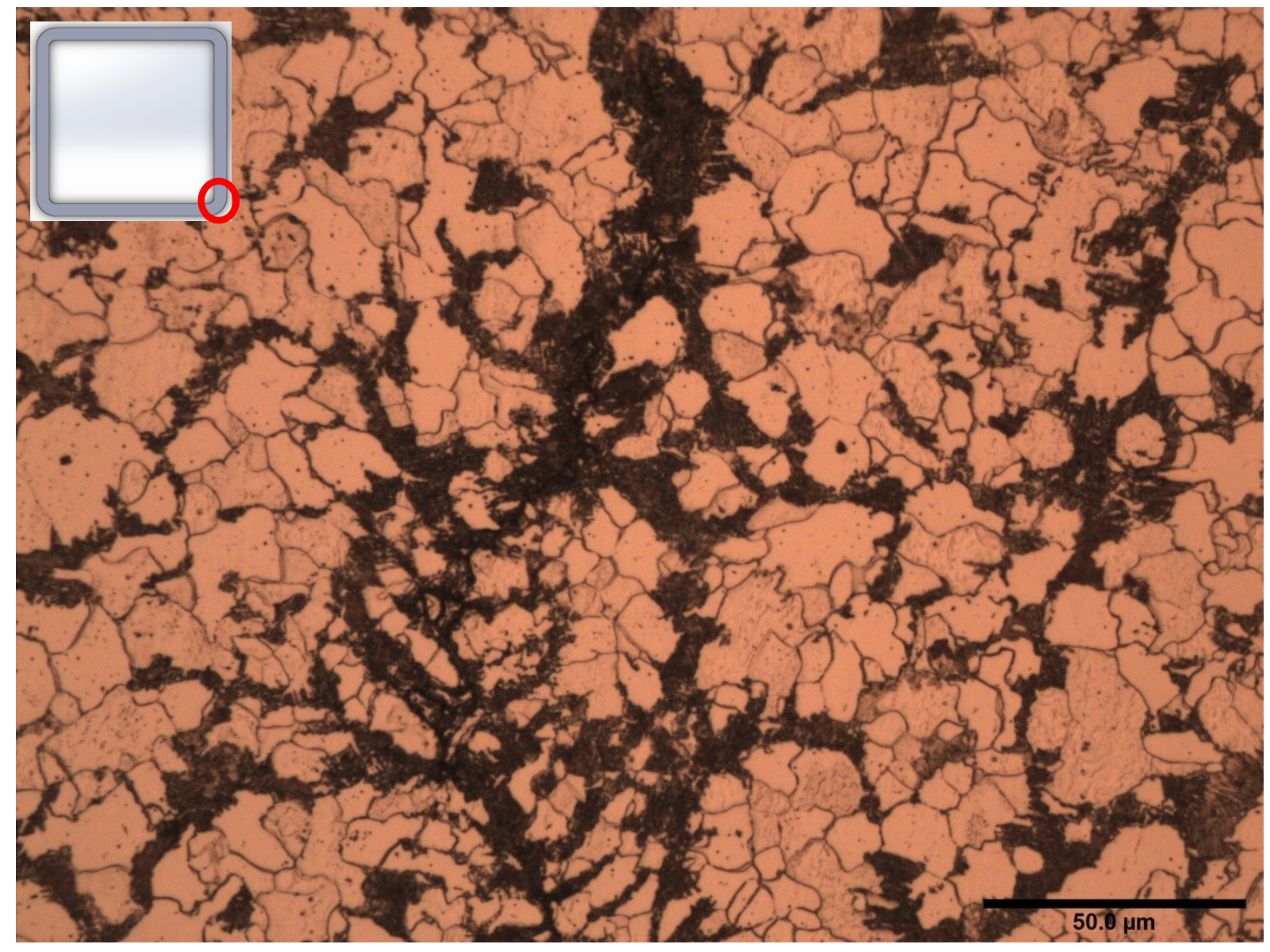

Figure 33-Hot-finished sample bent to $0.25 \%$ strain, intrados corner weld-line pushout, magnification $500 \mathrm{x}-\mathrm{H} 0.25 \mathrm{H} 6$

Figure 33 shows the low strain, weld-line push-out microstructure of the TATA Steel hot finished RHS at 500x magnification. The micrograph shows the collection of non-metallic inclusions on the weldline which is not uncommon. There does not seem to be any significant difference in grain size or shape when compared to the bulk material. The collection will have been caused during the welding process. Any impurities on the strip edges would have been ejected from the weld-line when the super-heated surfaces were pushed together, these impurities collected in the pushout. There is no evidence of any effects of cold working caused by the roller bending process. 


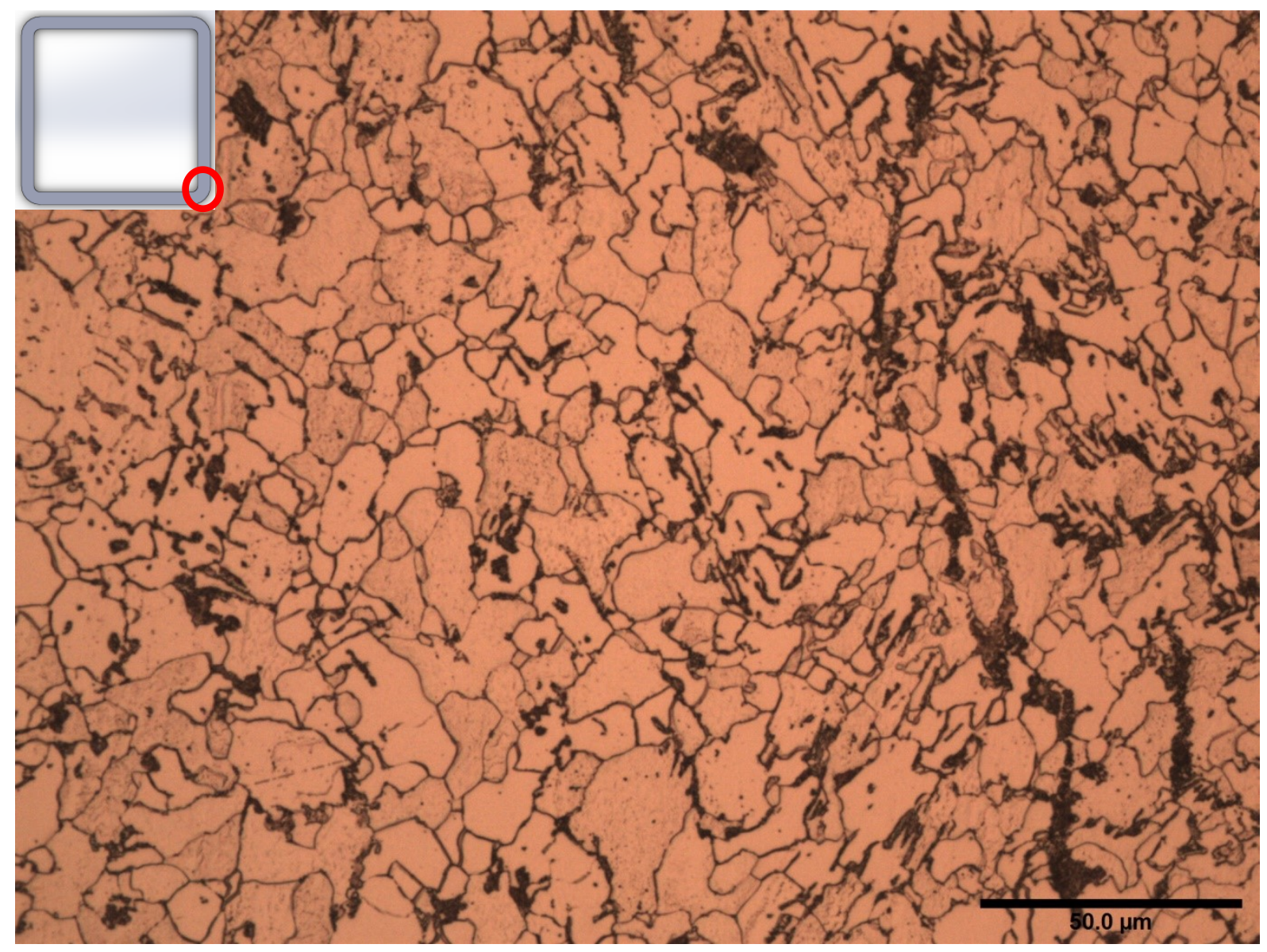

Figure 34-Hot-finished sample bent to $0.25 \%$ strain intrados corner weld-line pushout, magnification 500x-H0.25H6

Figure 34 is from the same sample as Figure 30 but the micrograph was taken closer to the edge of the push-out. There is a clear difference in the microstructure to Figure 30 , most likely caused by the more rapid cooling experienced by the protruding pushout. There is no evidence of the effects of cold working caused by the roller bending process. 


\section{High Strain Body}

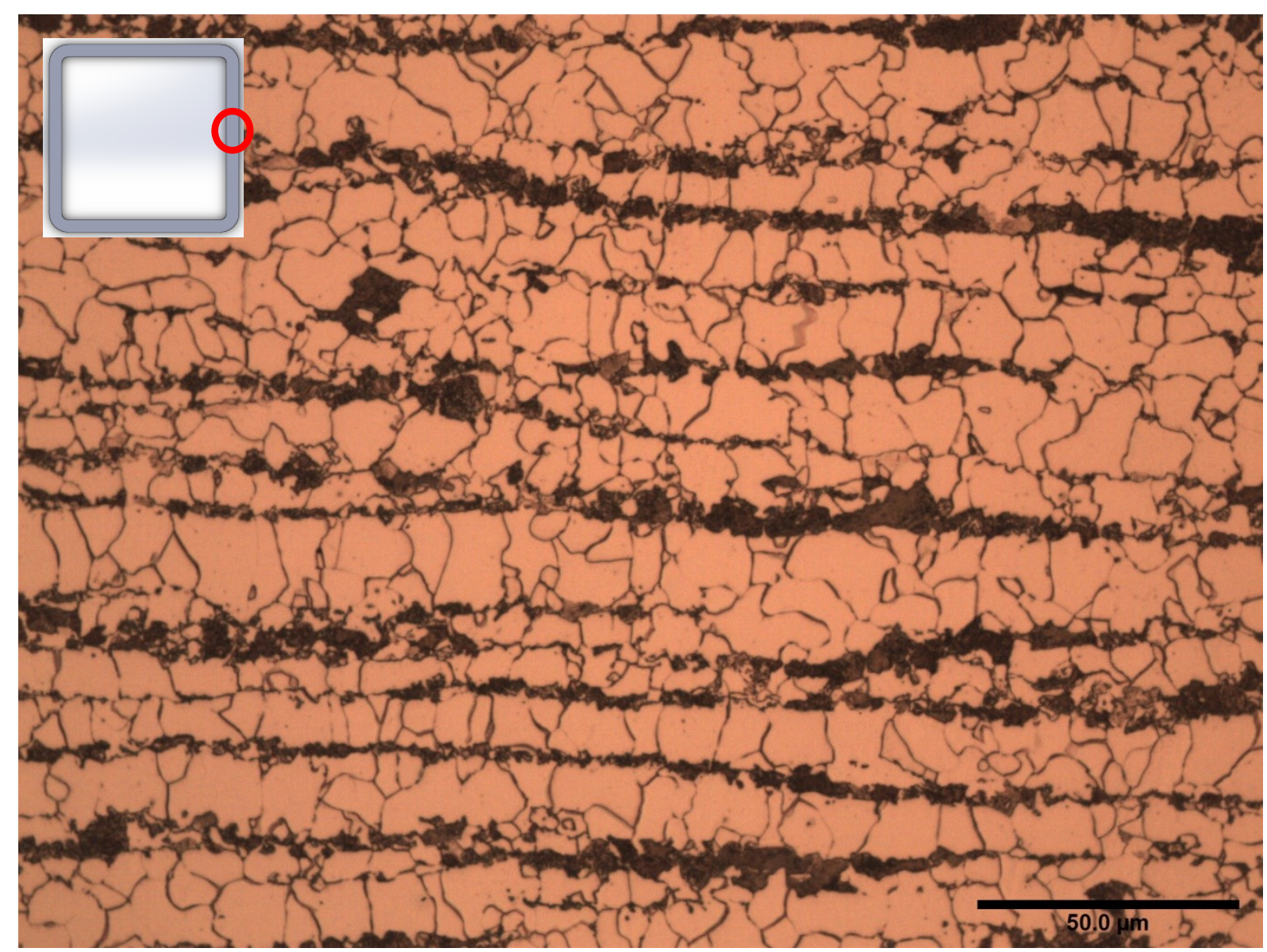

Figure 35-Hot-finished sample bent to 5\% strain, intrados flange, magnification 500x-H5.00L61

Figure 35 shows the high strain, intrados body microstructure of the TATA Steel hot finished RHS at 500x magnification. There seems to be some evidence of cold working caused by the roller bending process in that the grains appear to be slightly "taller" than the low strain equivalent shown in Figure 28 , presumably related to bending in the $Y-Y$ direction. 


\section{High Strain Corner}

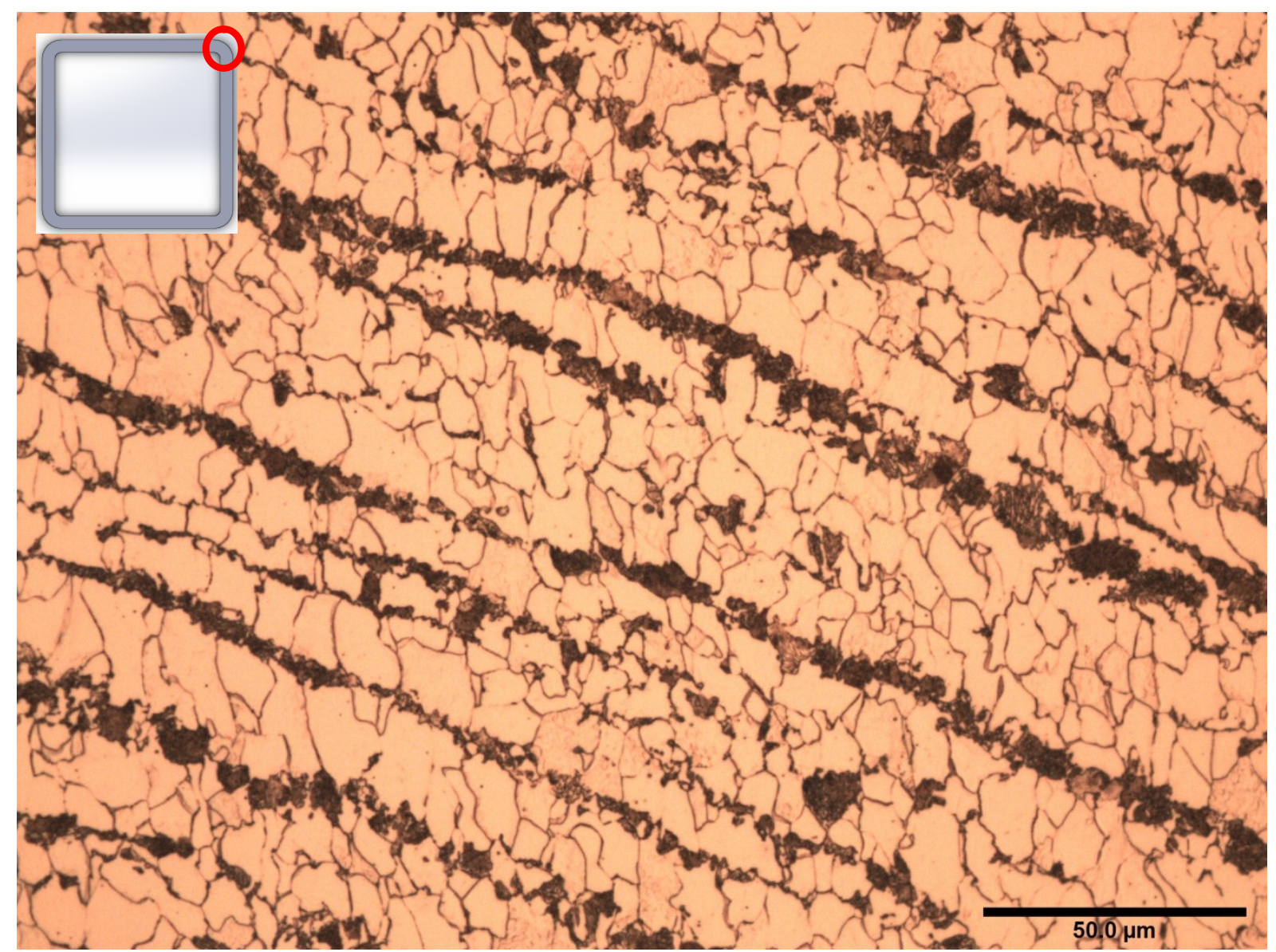

Figure 36-Hot-finished sample bent to $5 \%$ strain, extrados corner, magnification 500x-H5.00L65

Figure 36 shows the high strain, intrados corner microstructure of the TATA Steel hot finished RHS at 500x magnification. The change in orientation and grain elongation can clearly be seen when compared to Figure 29. The radius of the corner has also been significantly reduced as a result of the pressure applied by the bending rollers. 


\section{High Strain Weld Region}

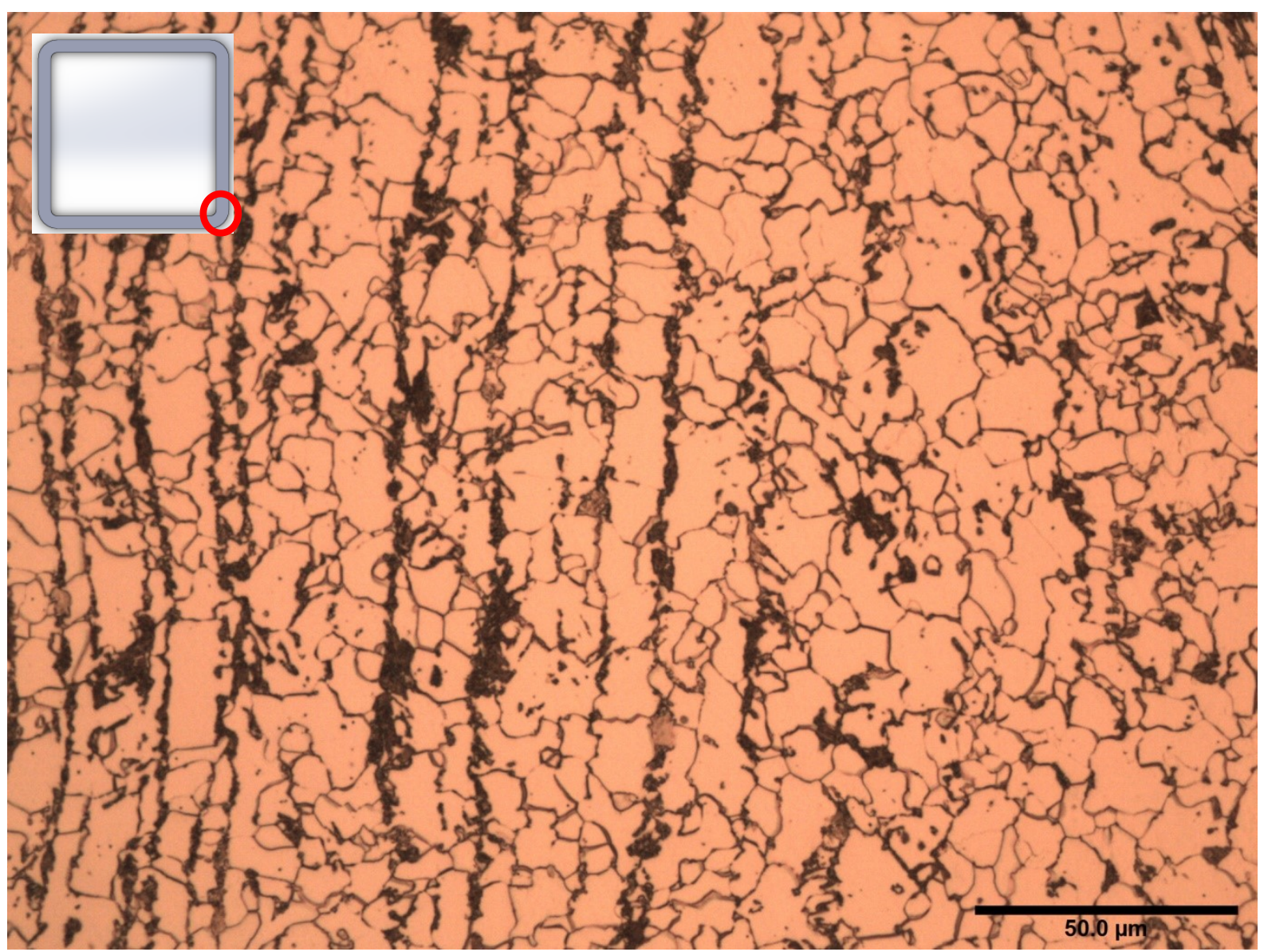

Figure $37-$ Hot-finished sample bent to $5 \%$ strain, intrados corner weld-line, magnification $500 x-$ H5.00L66

Figure 37 shows the high strain, weld region microstructure of the TATA Steel hot finished RHS at 500x magnification. There is no clear change in microstructure when compared to the low strain sample, Figure 31. 


\section{High Strain Pushout}

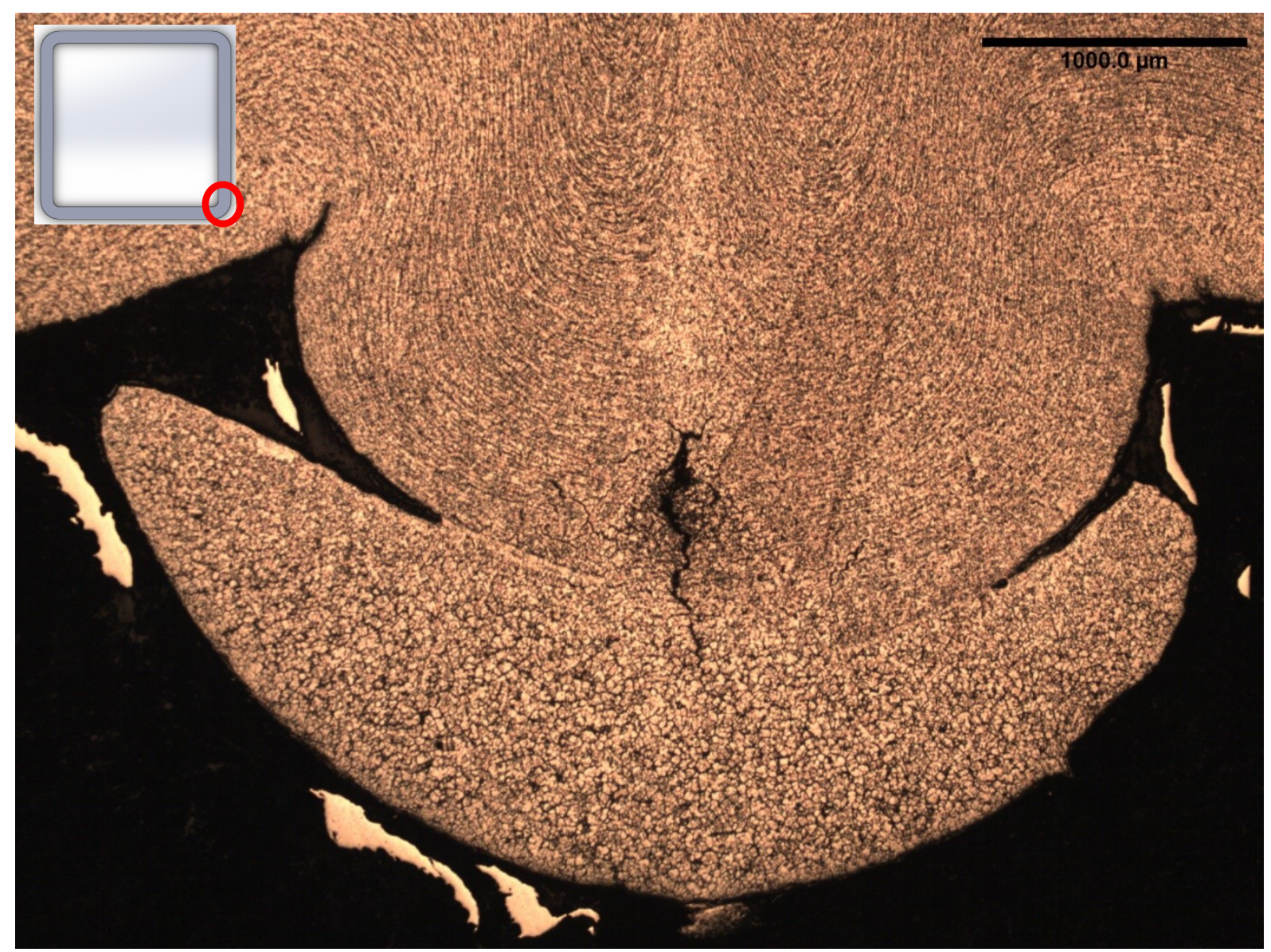

Figure 38-Hot-finished sample bent to $5 \%$ strain, intrados corner weld-line push-out, magnification $25 x-H 5.00 L 66$

Figure 38 shows the high strain, weld-line push-out microstructure of the TATA Steel hot finished RHS at $25 x$ magnification. The diversion of the banding adjacent to the weld-line can be seen clearly. The non-metallic inclusions noted in Figure 32 can still be seen towards the centre of the push-out. 


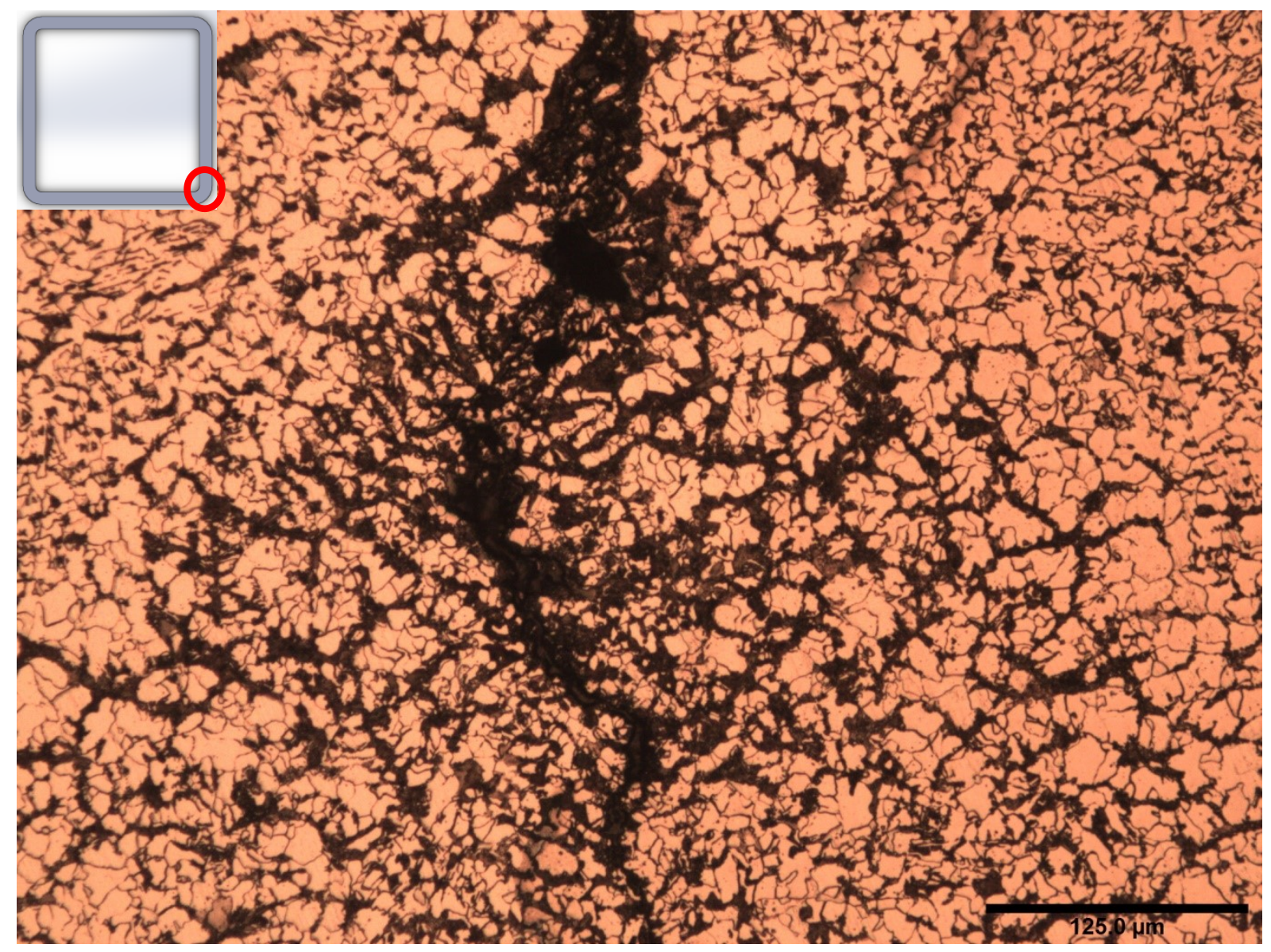

Figure $39-$ Hot-finished sample bent to $5 \%$ strain, intrados corner weld-line push-out, magnification

\section{0x-H5.00L66}

Figure 39 shows the high strain, weld-line pushout microstructure of the TATA Steel hot finished RHS at 200x magnification. The micrograph shows the region containing the non-metallic inclusions. However, there does not seem to be any difference in grain size or shape when compared to the bulk material. 


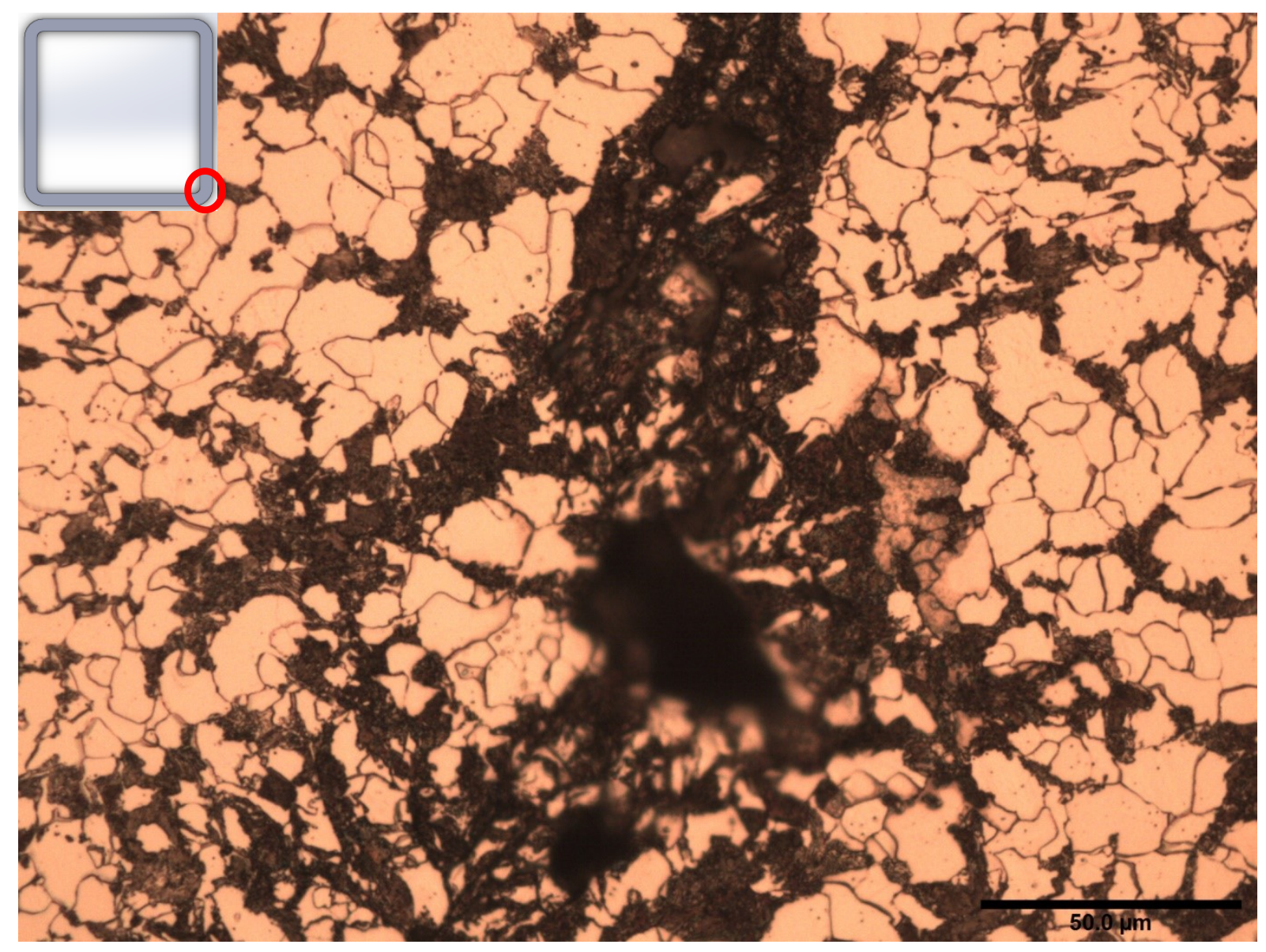

Figure 40 -Hot-finished sample bent to $5 \%$ strain, intrados corner weld-line push-out segregate void, magnification 500x-H5.00L66

Figure 40 shows the high strain, weld-line pushout microstructure of the TATA Steel hot finished RHS at 500x magnification. In addition to the non-metallic inclusions it can be seen that a void is also present. It is not clear whether this has been caused by the bending process or a poor "side squeeze" during the welding process. No other signs of cold work are apparent in this sample. 


\subsubsection{Cold-formed Square Hollow Section}

\section{Low Strain Body}

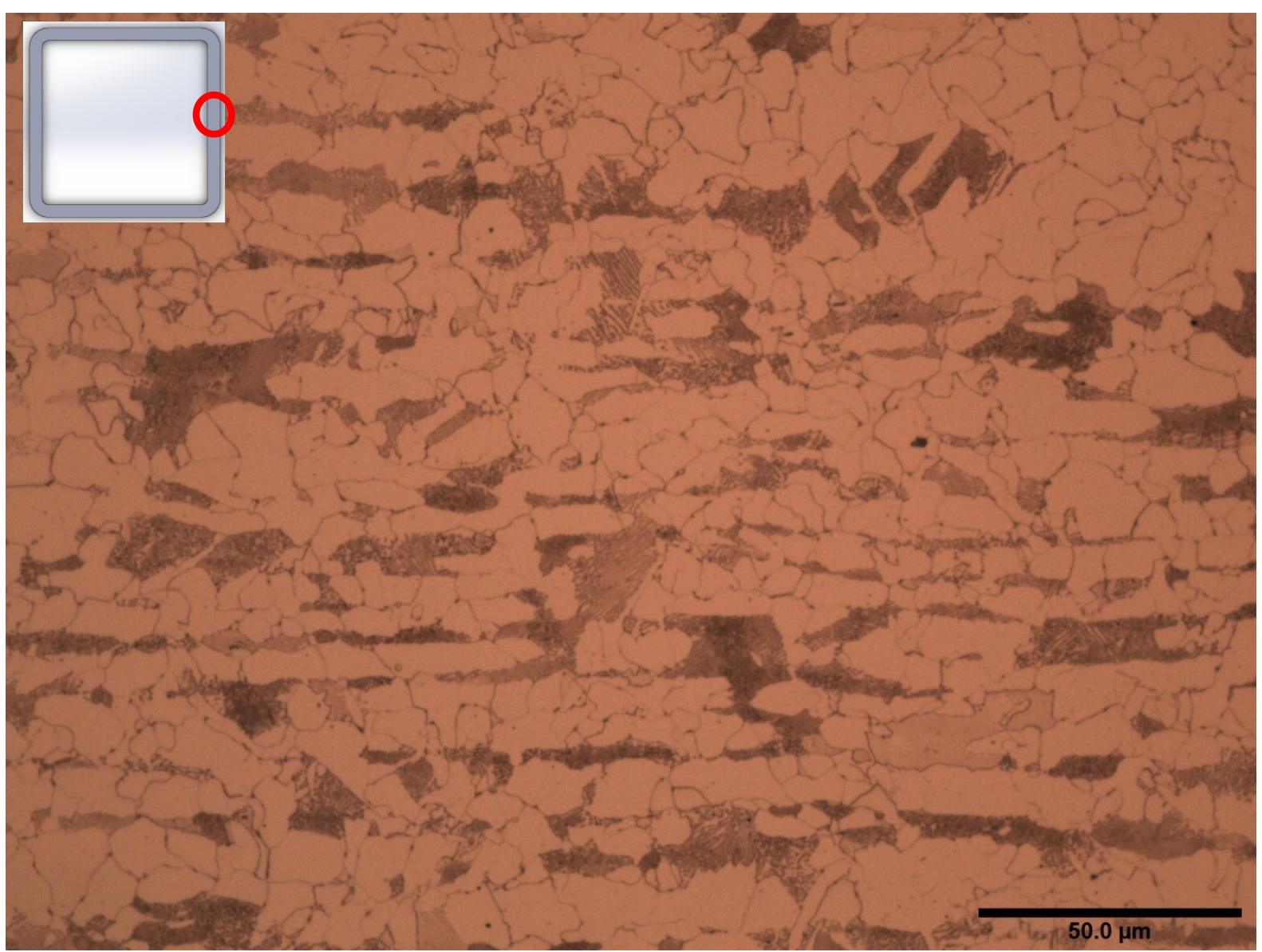

Figure 41-Cold-formed sample bent to $0.25 \%$ strain, intrados flange, magnification $500 x-\mathrm{C} 0.25 \mathrm{H} 1$

Figure 41 shows the low strain, body microstructure of the cold formed RHS at 500x magnification. The sample has a slightly grain oriented microstructure with transverse elongation apparent around the perimeter of the box-section with Pearlite banding throughout. There is also no evidence of further cold working effects as a result of the roller bending process. 


\section{Low Strain Corner}

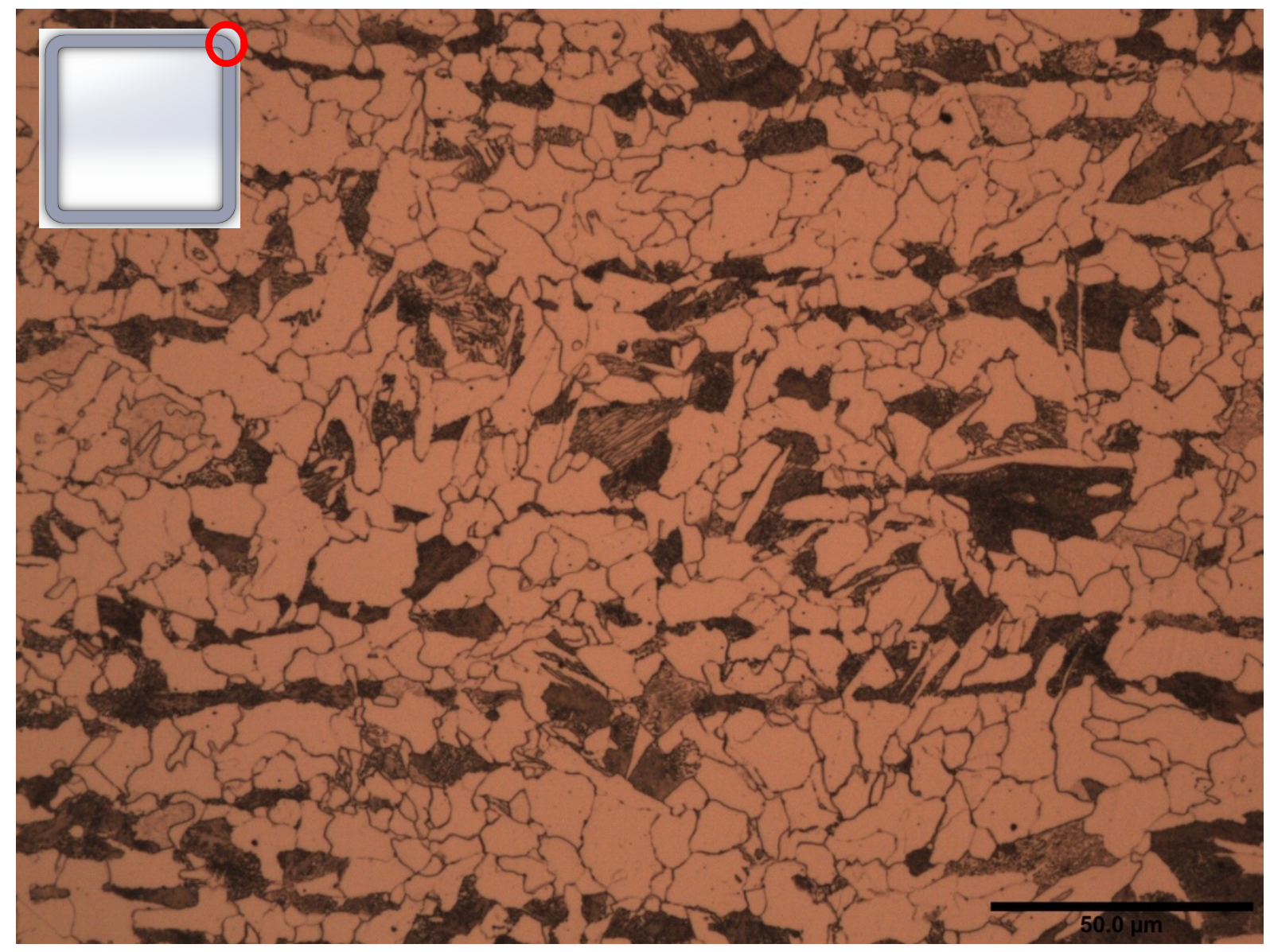

Figure 42-Cold-formed sample bent to $0.25 \%$ strain, intrados corner, magnification $500 x-\mathrm{C} 0.25 \mathrm{H} 4$

Figure 42 shows the low strain, intrados corner microstructure of the cold formed RHS at 500x magnification. There is no obvious grain orientation and although the grain sizes do vary significantly. There is no evidence of further cold working effects as a result of the roller bending process. 


\section{Low Strain Weld-line and HAZ}

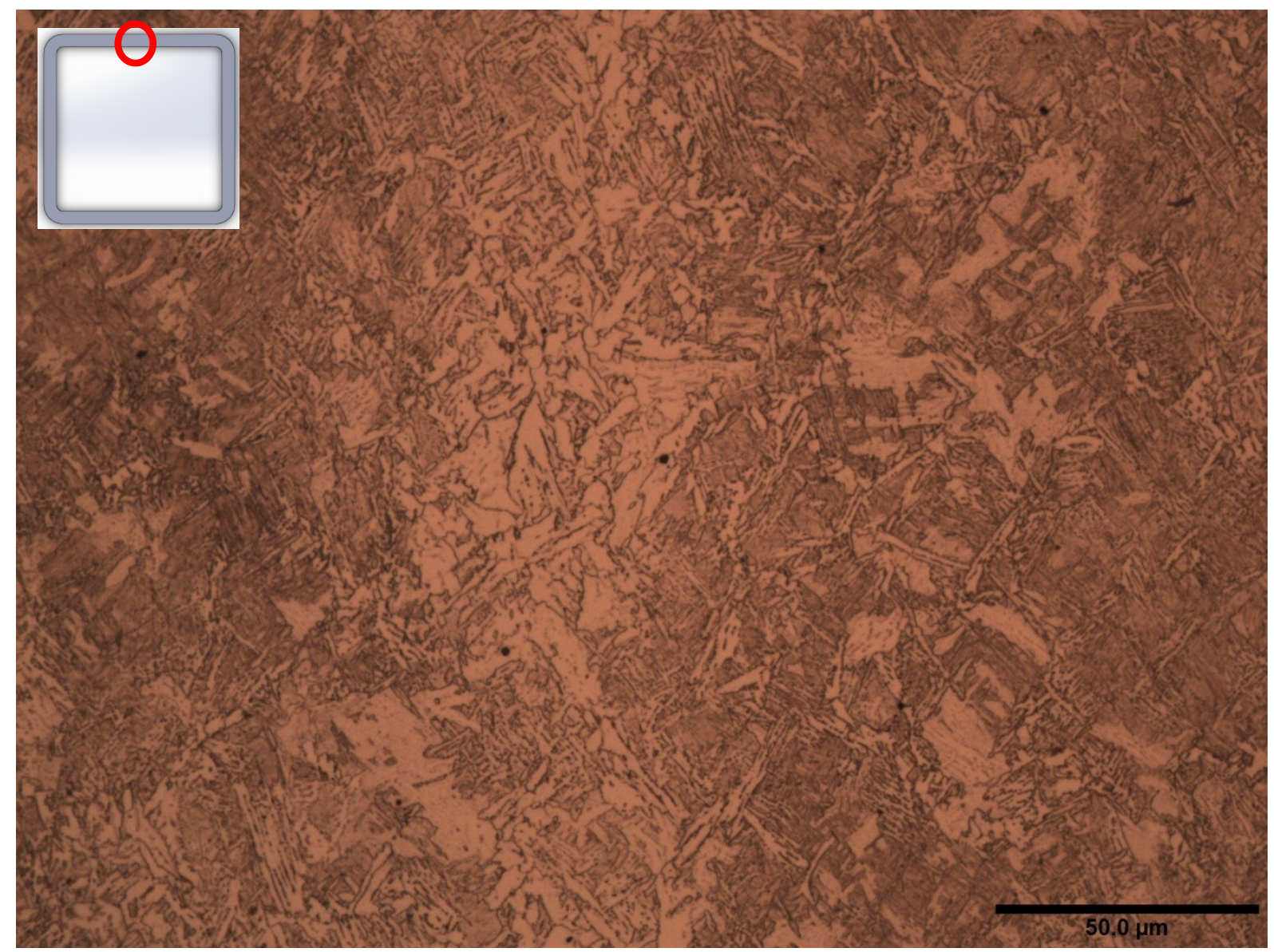

Figure 43 -Cold-formed sample bent to $0.25 \%$ strain, neutral axis weld-line, magnification $500 x$ -

\section{$\mathrm{C} 0.25 \mathrm{H} 2$}

Figure 43 shows the low strain, weld-line microstructure of the cold-formed RHS at 500x magnification. The weld-line has a fine grained, acicular ferrite microstructure. The presence of Martensite cannot be confirmed from this micrograph, higher magnifications would be required in order to do this. This type of microstructure is normally brought about by the rapid cooling after the weld has been formed. Such a microstructure is likely to be harder and more brittle than the body of the tube and would therefore be likely to demonstrate different mechanical property values. There is no clear evidence of further cold working effects as a result of the roller bending process. 


\section{Low Strain Push-out}

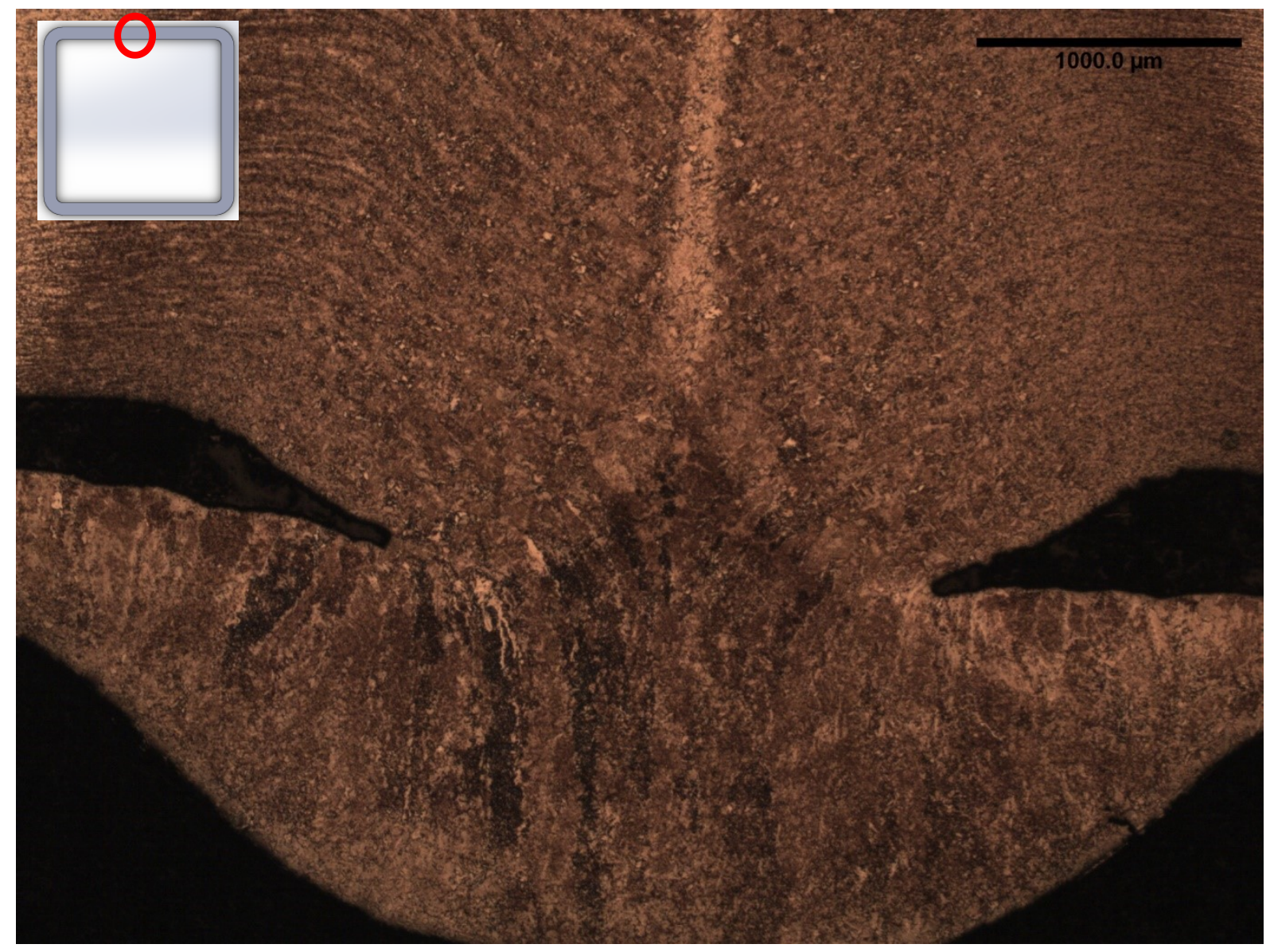

Figure 44 -Cold-formed sample bent to $0.25 \%$ strain, neutral axis weld-line push-out, magnification $25 x-C 0.252$

Figure 44 shows the low strain, weld-line push-out microstructure of the cold formed RHS at $25 x$ magnification. The deviation in the banding adjacent the weld-line can be seen clearly. The HAZ is also clearly visible either side of the weld-line. 


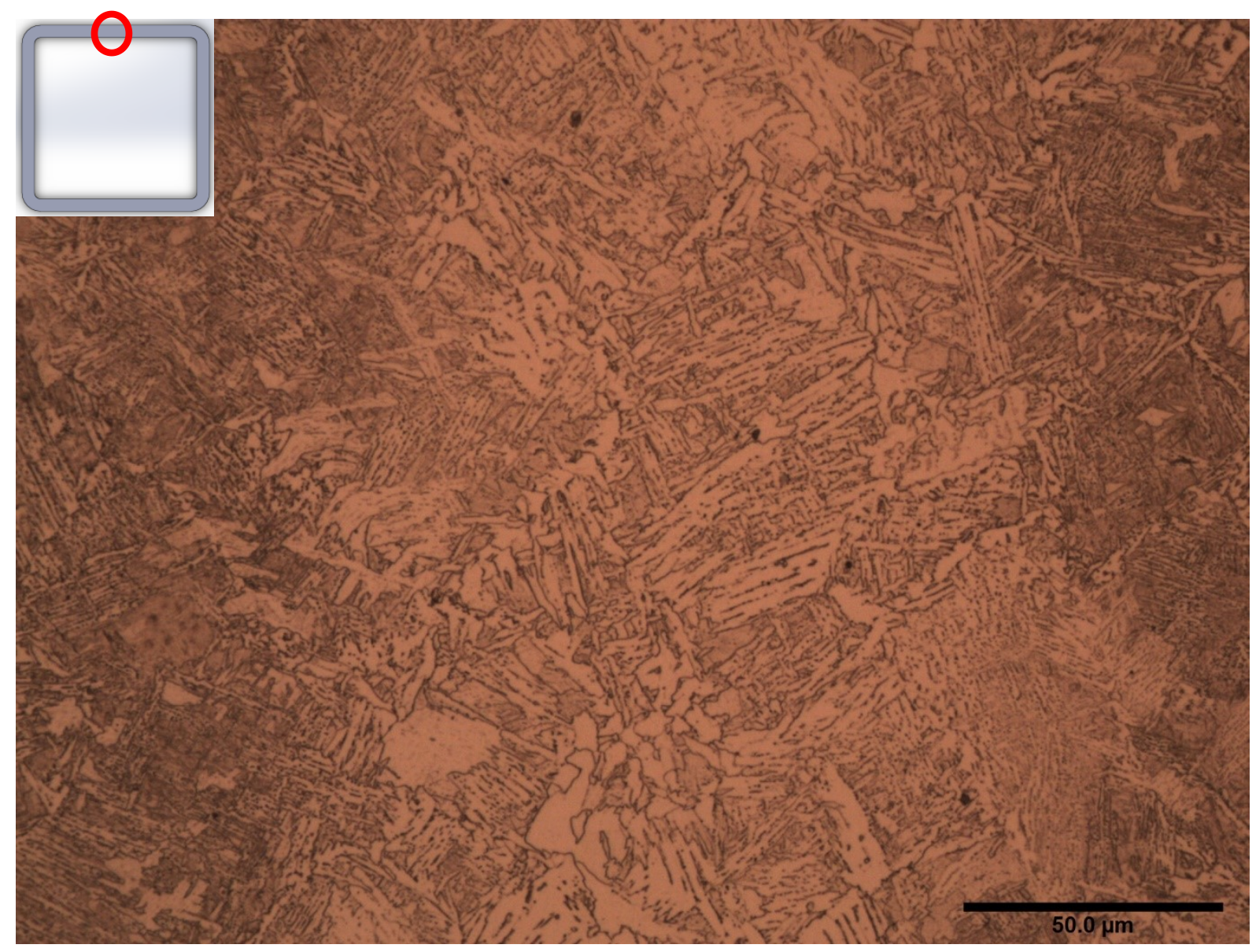

Figure 45 -Cold-formed sample bent to $0.25 \%$ strain, neutral axis weld-line push-out, magnification $500 x-C 0.25 \mathrm{H} 2$

Figure 45 shows the low strain, weld-line pushout microstructure of the cold-formed RHS at 500x magnification. The push-out has a fine grained, Widmanstätten ferrite microstructure. The presence of Martensite cannot be determined from this micrograph; a higher magnification would be required in order to confirm the presence of a martensitic microstructure. This microstructure is typical of that resulting from rapid cooling after welding. This microstructure would suggest that the HAZ is likely to be harder and more brittle than the body of the tube. There is no evidence of any effects of cold working caused by the roller bending process in this sample. 


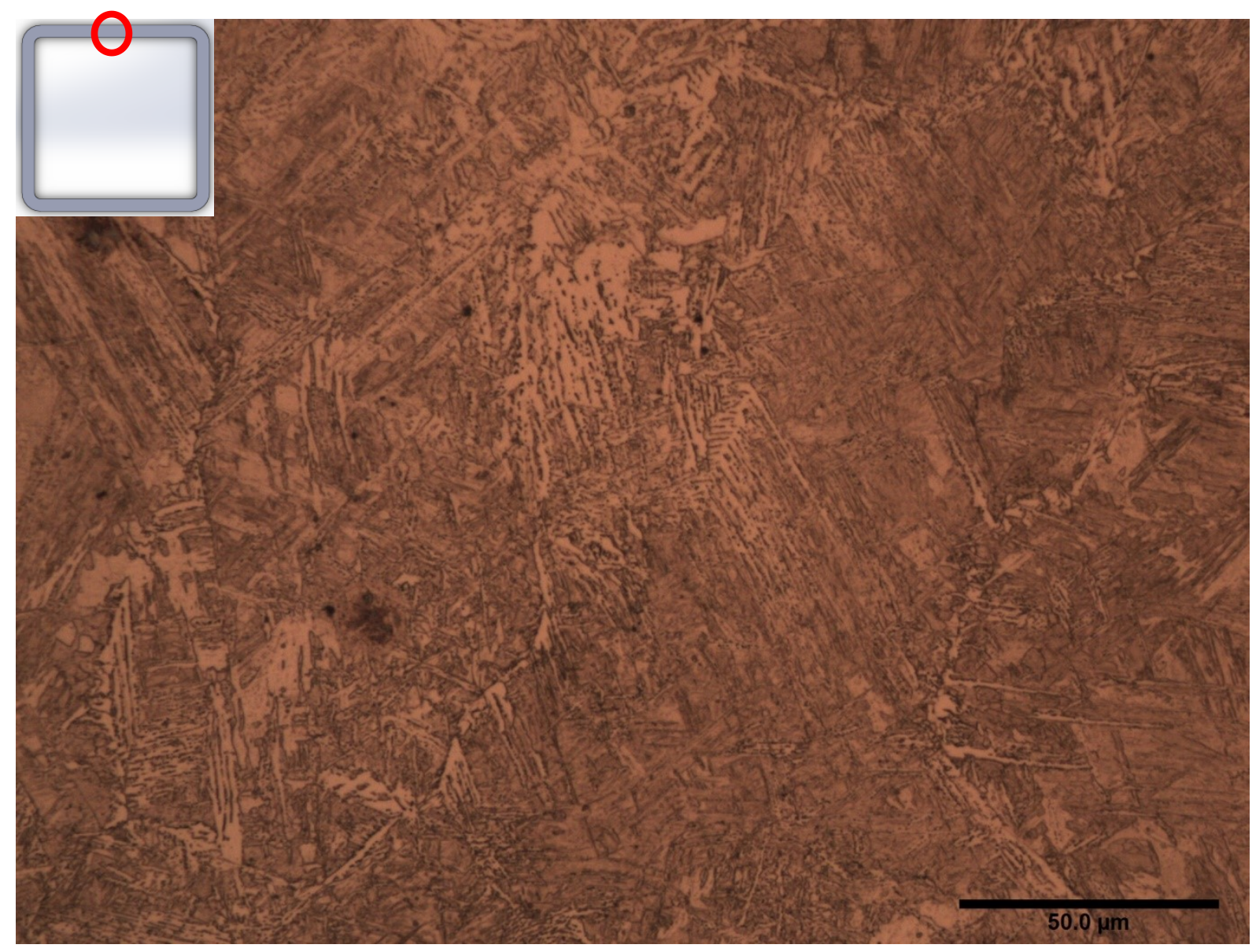

Figure 46 -Cold-formed sample bent to $0.25 \%$ strain, neutral axis weld-line push-out, magnification $500 x-C 0.25 \mathrm{H} 2$

Figure 46 also shows the low strain, weld-line pushout microstructure of the cold- formed RHS at 500x magnification. This photo micrograph was taken from closer to the edge of the push-out, an area likely to be subjected to an increased cooling rate after welding. This has yielded a fine grained, Widmanstätten ferrite microstructure. The presence of Martensite cannot be confirmed from this micrograph; however, due to the very rapid cooling experienced in this area it is likely that it is present. This microstructure would suggest that the HAZ is likely to be harder and more brittle than the body of the tube. There is no clear evidence of the effects of cold working caused by the roller bending process on the weld-line. 


\section{High Strain Body}

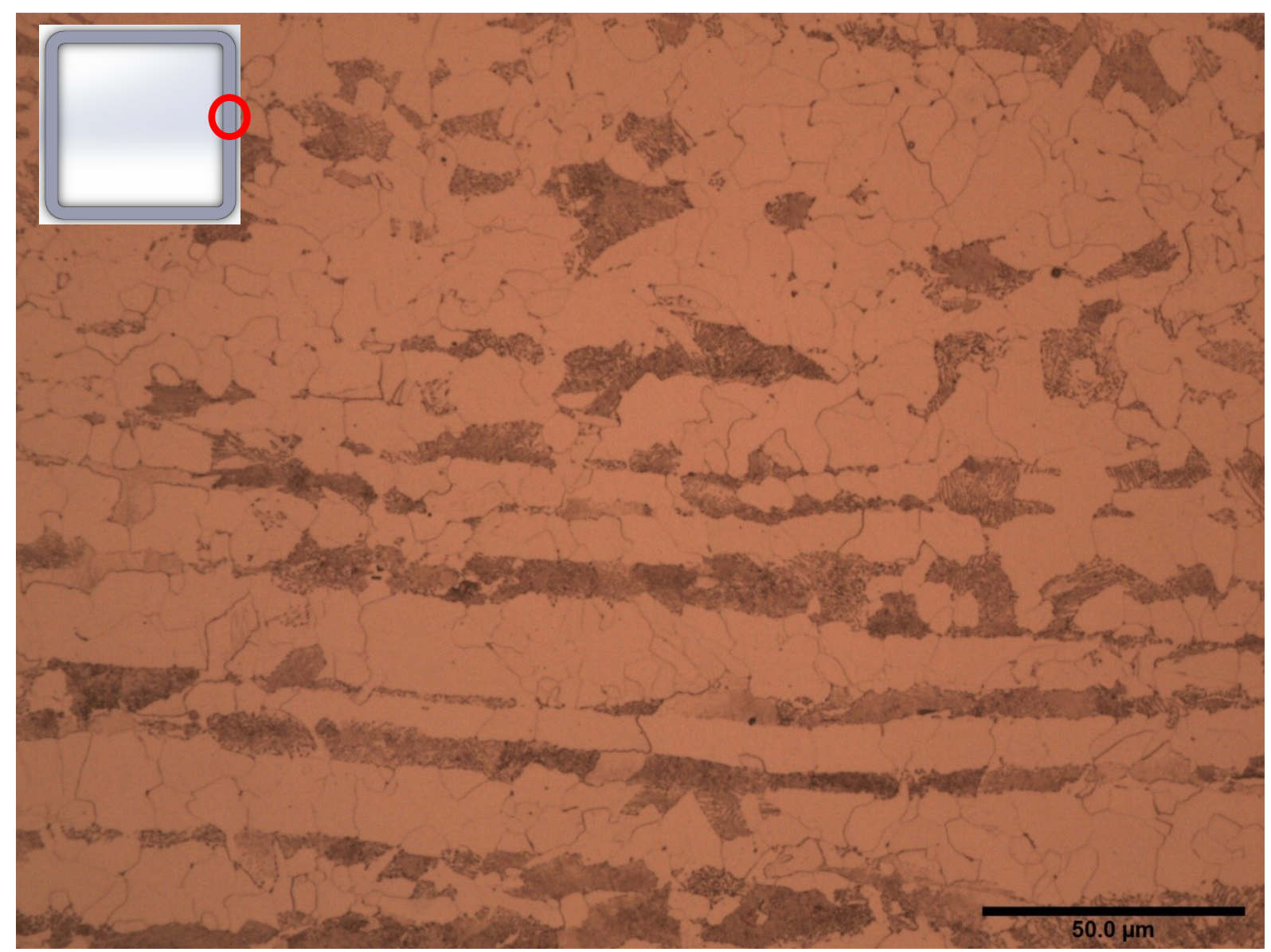

Figure 47-Cold-formed sample bent to 5\% strain, intrados flange, magnification 500x-C5.00L31

Figure 47 shows the high strain, intrados body microstructure of the cold-formed RHS at 500x magnification. There is no clear evidence from the microstructure of the effect of cold working caused by the roller bending process. 


\section{High Strain Corner}

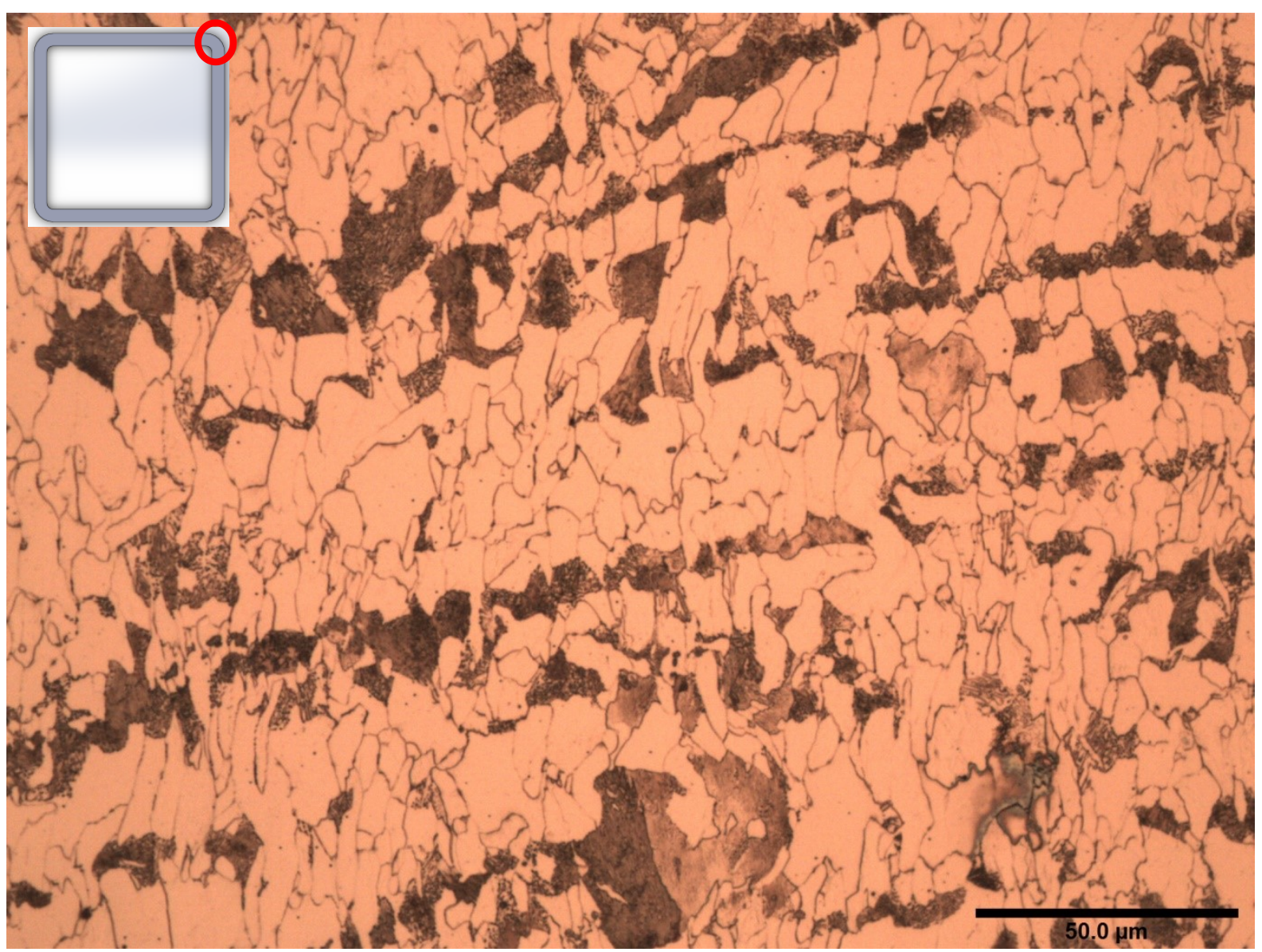

Figure 48-Cold-formed sample bent to 5\% strain, intrados corner, magnification 500x-C5.00L34

Figure 48 shows the high strain, intrados corner microstructure of the cold-formed RHS at 500x magnification. The change in orientation and grain elongation can clearly be seen when compared to Figure 42 . The radius of the corner has also been reduced slightly by the bending process. There also appears to be a small crack at a grain boundary in the bottom right of the micrograph. A possible reason for this could be the high stresses experienced in this area. The cause of this sharpening at the intrados corner is the pressure applied by the bending rollers. This leads to deformation of the malleable hot-finished box section into the shape of the roller profile. 


\section{High Strain Weld-line and HAZ}

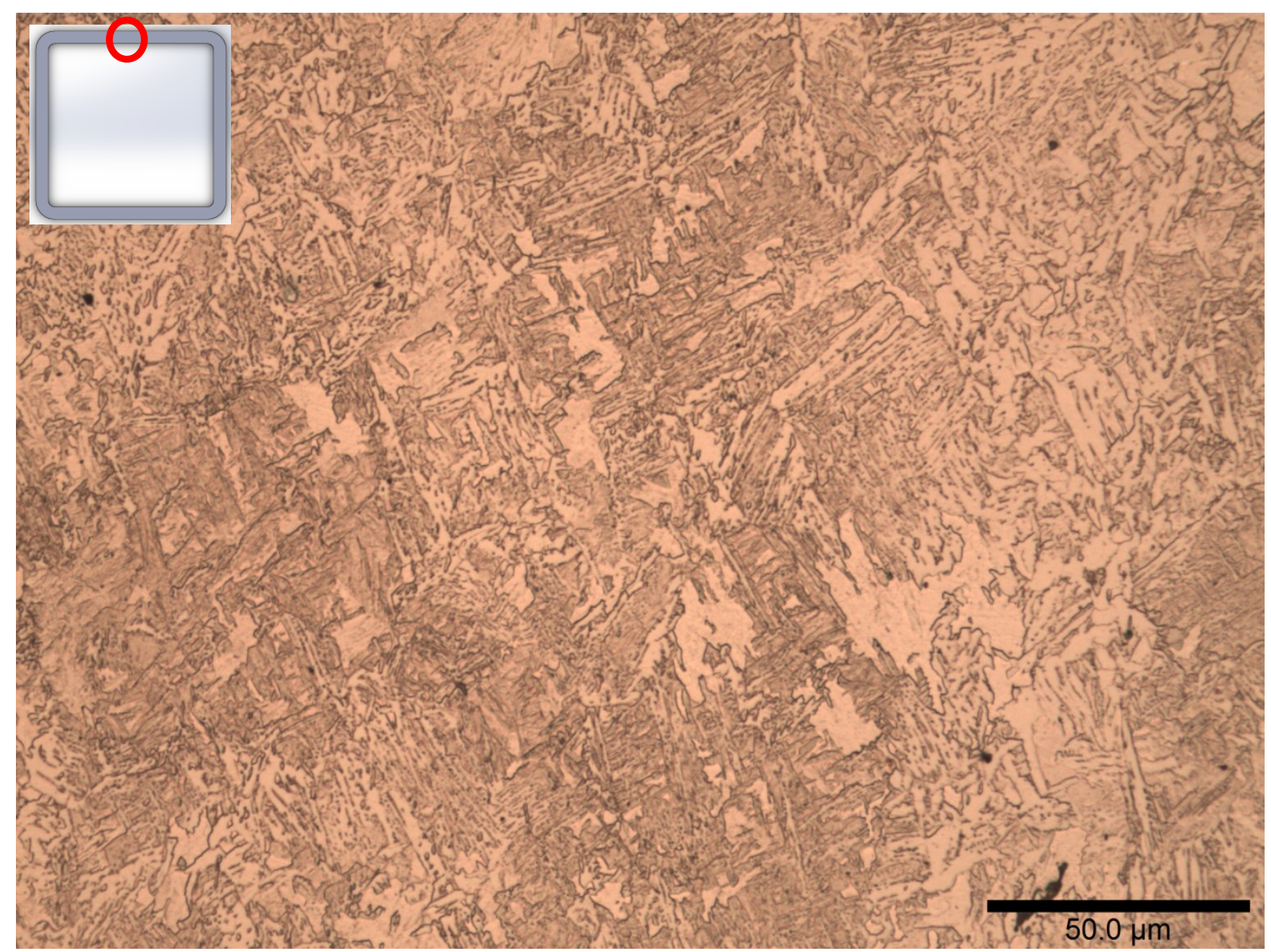

Figure 49 -Cold-formed sample bent to $5 \%$ strain, neutral axis weld-line, magnification 500x-

\section{C5.00L32}

Figure 49 shows the high strain, weld-line and HAZ microstructure of the cold- formed RHS at 500x magnification. The weld-line has a fine grained, acicular ferrite microstructure. The HAZ to the left of the weld-line (shown to the right) appears to comprise Widmanstätten ferrite. The presence of Martensite cannot be confirmed from this micrograph, higher magnifications would be required in order to detect the presence of a martensitic microstructure. This structure is typically formed by the rapid cooling experienced in the area after welding. This microstructure would suggest that the HAZ is likely to be harder and more brittle than the body of the tube. The structure is such that no clear evidence of the cold working caused by the roller bending process can be seen. 


\section{High Strain Push-out}

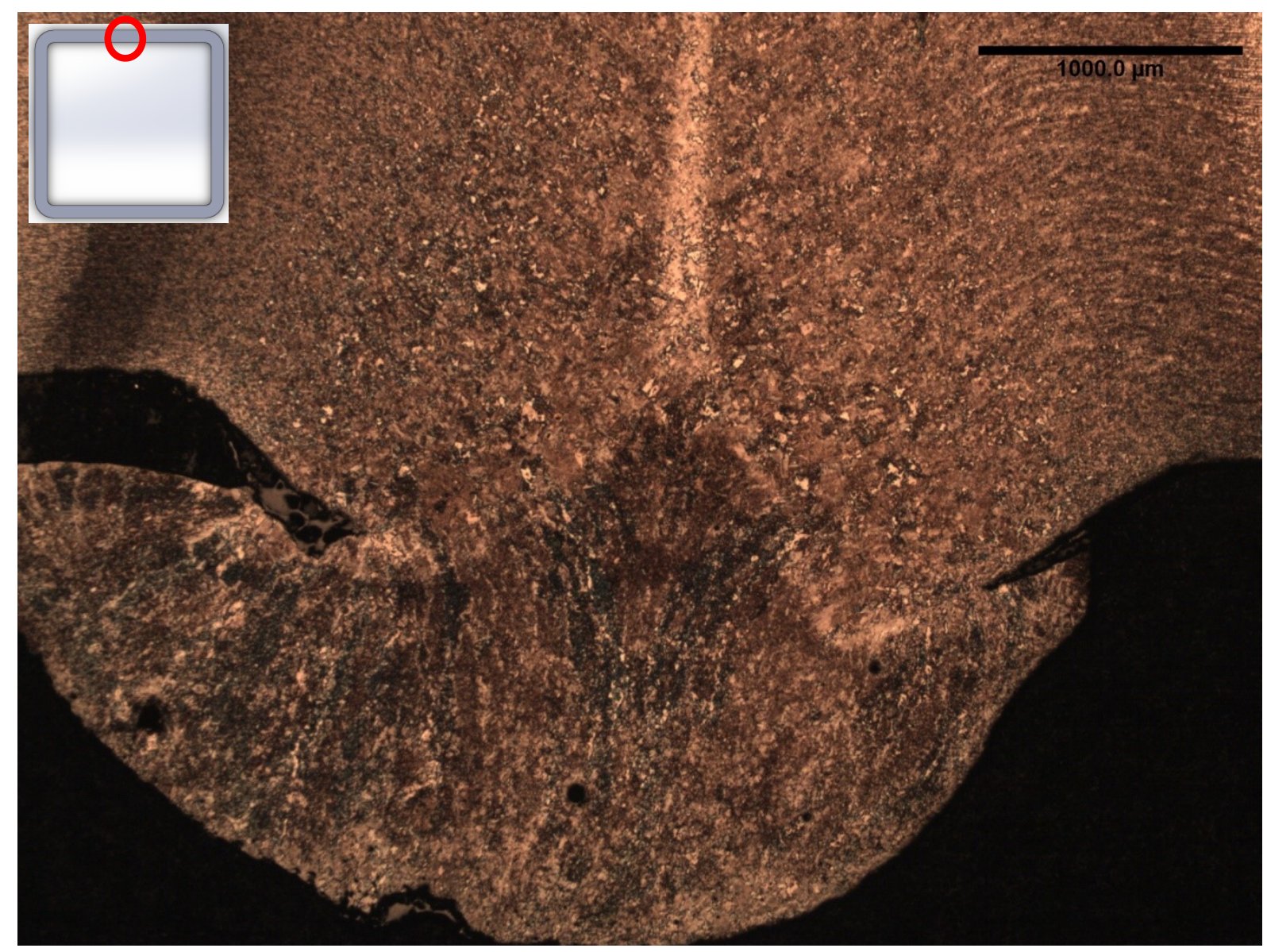

Figure 50 -Cold-formed sample bent to $5 \%$ strain, neutral axis weld-line push-out, magnification $25 x-C 5.00 L 32$

Figure 50 shows the high strain, weld-line pushout microstructure of the cold formed RHS at $25 x$ magnification. The deviation in the banding adjacent to the weld-line can be seen clearly. The HAZ is also clearly visible either side of the weld-line. 


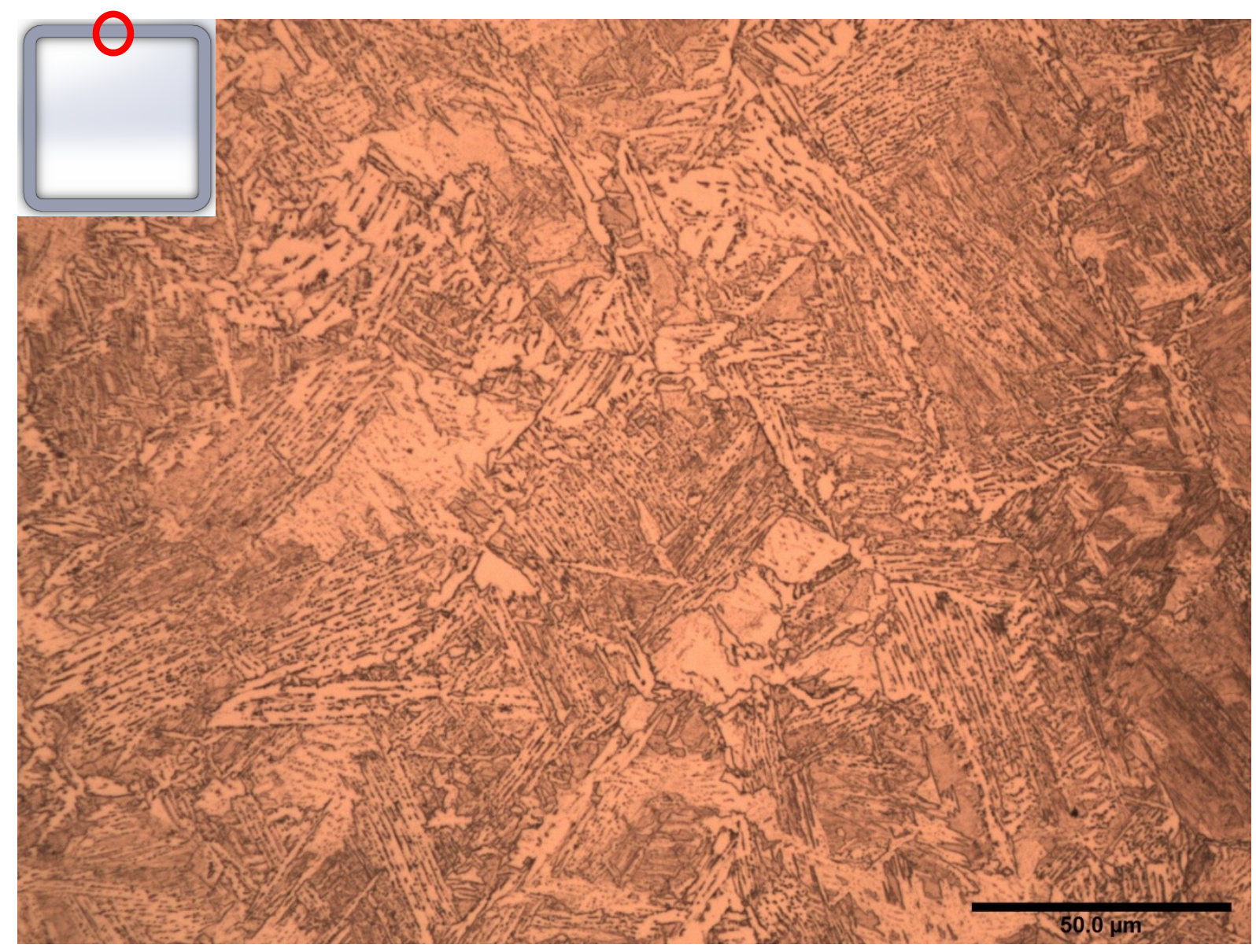

Figure 51 -Cold-formed sample bent to $5 \%$ strain, neutral axis weld-line pushout, magnification $500 \mathrm{x}-\mathrm{C} 5.00 \mathrm{~L} 32$

Figure 51 shows the high strain, weld-line pushout microstructure of the cold- formed RHS at 500x magnification. The push-out has a fine grained, acicular and Widmanstätten ferrite microstructure. The presence of Martensite cannot be confirmed from this micrograph; examination at higher magnifications would be required in order to detect the presence of a martensitic microstructure. Such a microstructure is typically caused by rapid cooling after welding. This microstructure would suggest that the HAZ is likely to be harder and more brittle than the body of the tube. There is no clear evidence of cold working caused by the roller bending process. 


\subsection{Hardness}

\subsubsection{Surface Hardness}

\subsubsection{Hot-finished Rectangular Hollow Section}

\begin{tabular}{|c|c|c|c|c|c|c|c|c|c|c|}
\hline \multirow{3}{*}{$\begin{array}{l}\text { Strain } \\
\text { Rate } \\
(\%)\end{array}$} & \multicolumn{3}{|c|}{ Intrados 1} & \multirow{3}{*}{$\begin{array}{l}\text { Intrados } \\
\text { Mean } \\
\text { (HV) }\end{array}$} & \multirow{3}{*}{$\begin{array}{l}\text { Intrados } \\
\text { SD } \\
\text { (HV) }\end{array}$} & \multicolumn{3}{|c|}{ Extrados 3} & \multirow{3}{*}{$\begin{array}{l}\text { Extrados } \\
\text { Mean } \\
\text { (HV) }\end{array}$} & \multirow{3}{*}{$\begin{array}{l}\text { Intrados } \\
\text { SD } \\
\text { (HV) }\end{array}$} \\
\hline & (HV) & & & & & (HV) & & & & \\
\hline & & & & & & & & & & \\
\hline 0.00 & 160 & 161 & 164 & 162 & 2.08 & 168 & 160 & 162 & 163 & 4.16 \\
\hline 0.25 & 172 & 170 & 166 & 169 & 3.06 & 160 & 157 & 163 & 160 & 3.00 \\
\hline 0.50 & 170 & 167 & 174 & 170 & 3.51 & 162 & 154 & 160 & 159 & 4.16 \\
\hline 1.00 & 176 & 172 & 177 & 175 & 2.65 & 165 & 163 & 166 & 165 & 1.53 \\
\hline 2.00 & 168 & 171 & 178 & 172 & 5.13 & 163 & 164 & 170 & 166 & 3.79 \\
\hline 3.00 & 179 & 173 & 173 & 175 & 3.46 & 160 & 173 & 166 & 166 & 6.51 \\
\hline 4.00 & 160 & 166 & 178 & 168 & 9.17 & 168 & 168 & 160 & 165 & 4.62 \\
\hline $4.00 / L 6$ & 179 & 181 & 183 & 181 & 2.00 & 184 & 172 & 180 & 179 & 6.11 \\
\hline $5.00 /$ L6 & 178 & 182 & 178 & 179 & 2.31 & 173 & 176 & 178 & 176 & 2.52 \\
\hline
\end{tabular}

Table 4-Surface hardness at the intrados and extrados of the hot-finished RHS

Table 4 shows the surface hardness of the TATA Steel hot-finished RHS at both the intrados and extrados as strain rate is increased. The surface hardness of the hot-finished rectangular hollow section is approximately $162 \mathrm{HV}$ at both the intrados and extrados before bending takes place.

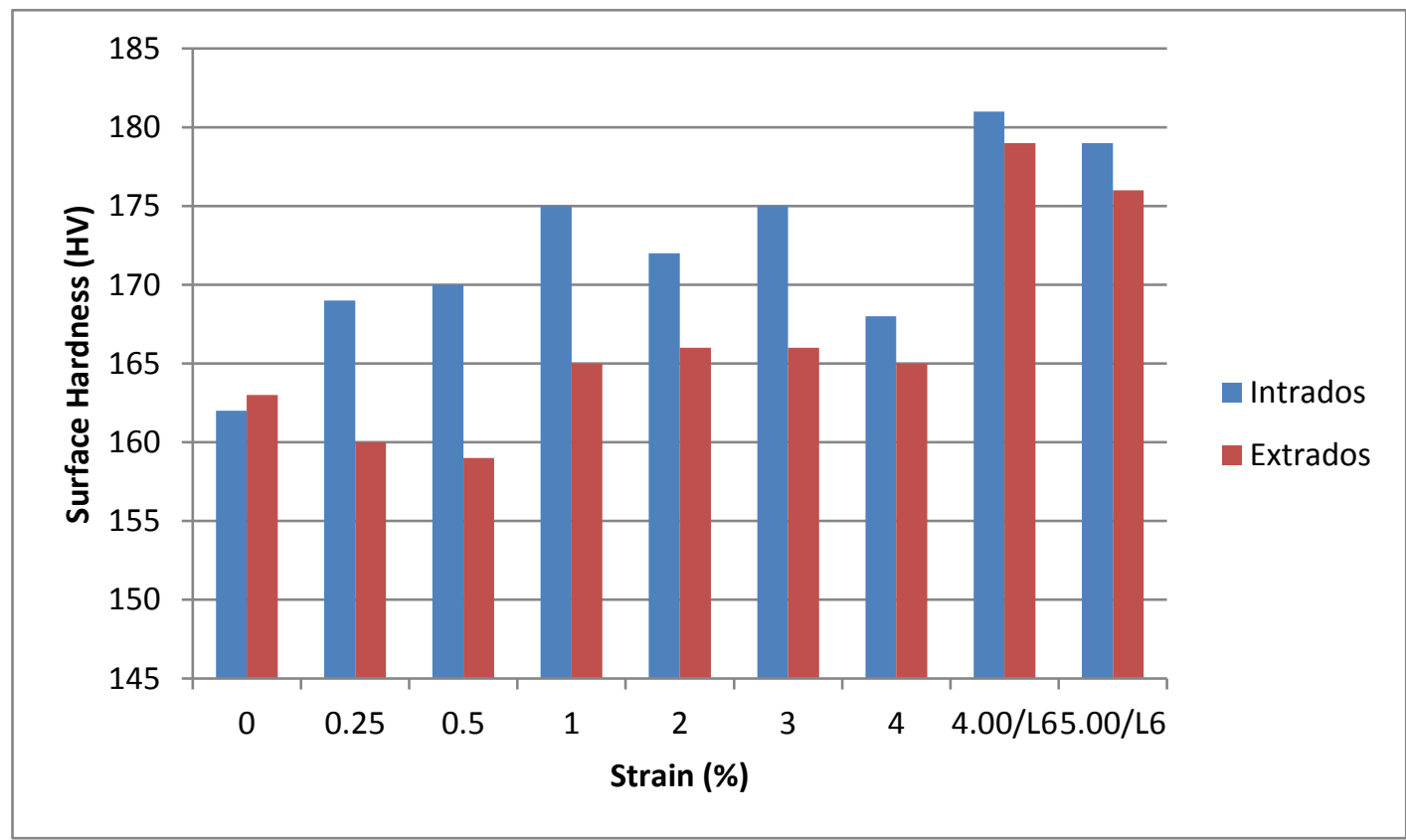

Figure 52-Graph plotting the hot-finished RHS surface hardness for the intrados and extrados with increasing strain 
The graph plots the intrados and extrados mean surface hardness for the hot-finished RHS. It is clear from Figure 52 that the intrados experiences an increase in hardness due to bending, however the extrados initially experiences a reduction in surface hardness before an increase is observed at $1 \%$ strain. More gradual bending of the tube affects surface hardness less than more rapid bending (with fewer passes through the equipment). Rapid bending produces the hardest surface microstructure at both the intrados and extrados. The maximum mean increase in hardness at the intrados is $19 \mathrm{HV}$ this was observed at $4 \%$ strain for the sample that was rapidly curved. This would indicate that it is not the strain alone that is critical but the rate at which the strain is applied. 


\subsubsection{Cold-formed Rectangular Hollow Section}

\begin{tabular}{|c|c|c|c|c|c|c|c|c|c|c|}
\hline \multirow{3}{*}{$\begin{array}{l}\text { Strain } \\
\text { Rate } \\
(\%)\end{array}$} & \multicolumn{3}{|c|}{ Intrados 1} & Intrados & Intrados & \multicolumn{3}{|c|}{ Extrados 3} & \multirow{3}{*}{$\begin{array}{l}\text { Extrados } \\
\text { Mean } \\
\text { (HV) }\end{array}$} & \multirow{3}{*}{$\begin{array}{l}\text { Intrados } \\
\text { SD } \\
\text { (HV) }\end{array}$} \\
\hline & (HV) & & & Mean & SD & (HV) & & & & \\
\hline & & & & (HV) & (HV) & & & & & \\
\hline 0.00 & 176 & 181 & 165 & 174 & 8.19 & 178 & 160 & 155 & 164 & 12.10 \\
\hline 0.25 & 185 & 182 & 173 & 180 & 6.24 & 165 & 182 & 166 & 171 & 9.54 \\
\hline 0.50 & 179 & 187 & 188 & 185 & 4.93 & 176 & 177 & 177 & 177 & 0.58 \\
\hline 1.00 & 181 & 177 & 184 & 181 & 3.51 & 183 & 188 & 177 & 183 & 5.51 \\
\hline 2.00 & 187 & 188 & 188 & 188 & 0.58 & 180 & 184 & 174 & 179 & 5.03 \\
\hline 3.00 & 184 & 186 & 176 & 182 & 5.29 & 170 & 180 & 176 & 175 & 5.03 \\
\hline 4.00 & 183 & 179 & 177 & 180 & 3.06 & 173 & 181 & 175 & 176 & 4.16 \\
\hline $4.00 / L 4$ & 196 & 190 & 189 & 192 & 3.79 & 188 & 185 & 186 & 186 & 1.53 \\
\hline $5.00 /$ L3 & 191 & 192 & 185 & 189 & 3.79 & 185 & 188 & 189 & 187 & 2.08 \\
\hline
\end{tabular}

Table 5-Surface hardness at the intrados and extrados of the cold-formed RHS

Table 5 shows the surface hardness of the cold formed RHS at both the intrados and extrados as strain rate is increased. There is no definitive evidence to suggest that the surface of the tube has been significantly affected by the roller bending process. 
The graph show that there is little change in surface hardness as strain is increased. There is a small surface hardness increase initially before the graph levels off and seems to be in decline by $3 \%$ strain at the intrados and between 1 and $2 \%$ strain at the extrados. In the majority of the results, the surface hardness at the intrados was greater than that of the extrados.

The maximum mean increase in hardness at the intrados is $18 \mathrm{HV}$ this was observed at $4 \%$ strain for the low pass number sample. At the extrados, the maximum increase in surface hardness was $23 \mathrm{HVat} 5 \%$ strain on the low pass number sample. This would indicate that it is not the strain that is critical but the rate at which the strain is applied.

The surface hardness of the cold-formed RHS was greater than that of the hot-finished RHS. The cold-formed section also had a higher variation in surface hardness of the original, unbent material with a range of measurements from approximately 150 to $180 \mathrm{HV}$.

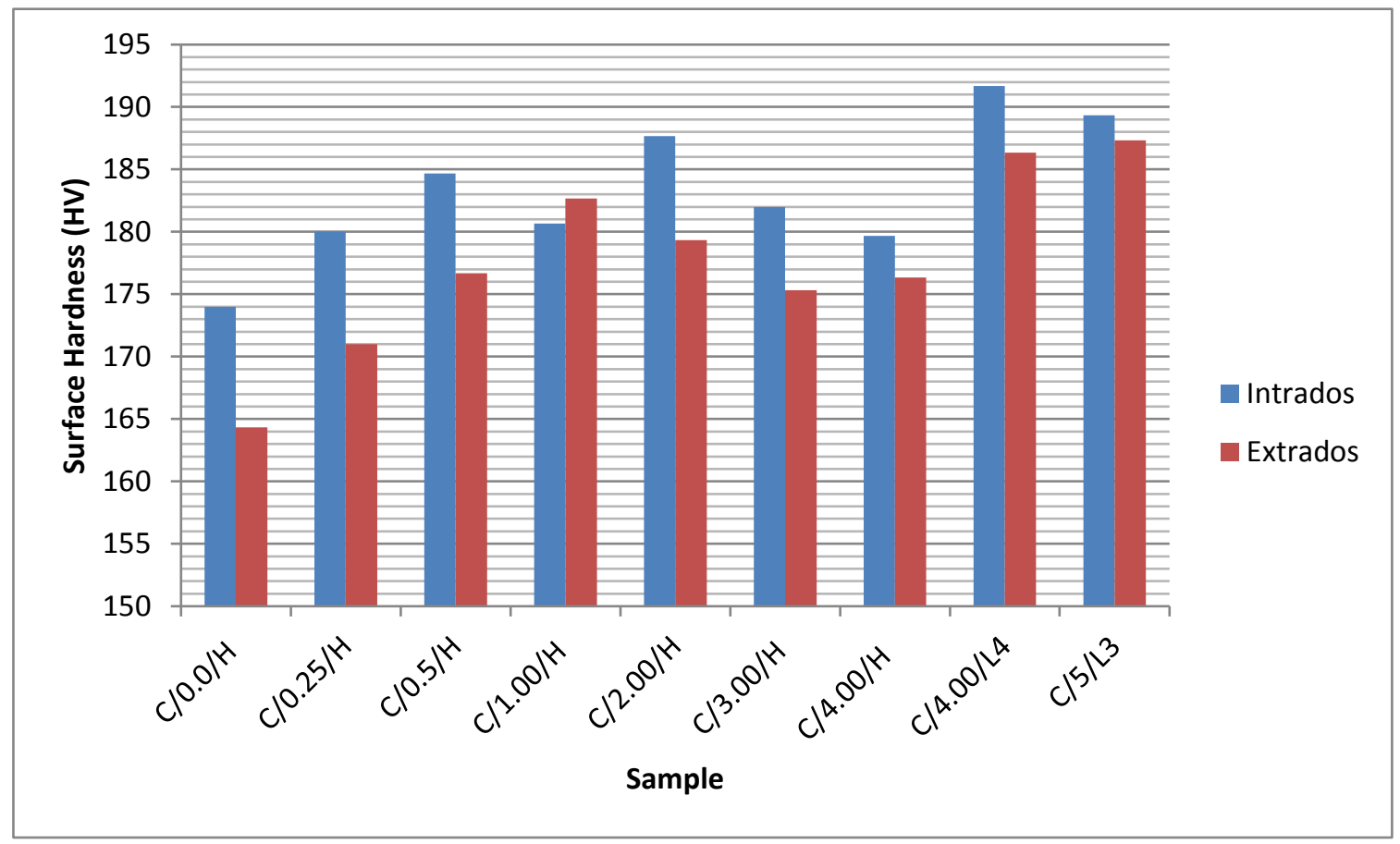

Figure 53-Graph plotting the intrados and extrados surface hardness of the cold-formed RHS as strain is increased 


\subsubsection{Cross-Section Hardness}

The hardness of the cross-section has been measured using an automatic hardness tester at each testing location (1-5 for the cold-formed and 1-6 for the hot-finished). At each location, a hardness profile has been measured for tree depths through the gauge $2 \mathrm{~mm}$ from the outside surface, at the centre line, and $2 \mathrm{~mm}$ from the inside surface. The results from each sample area have been compared to assess the effect of bending on the hardness of the tubes.

\subsubsection{Hot-finished Rectangular Hollow Section}

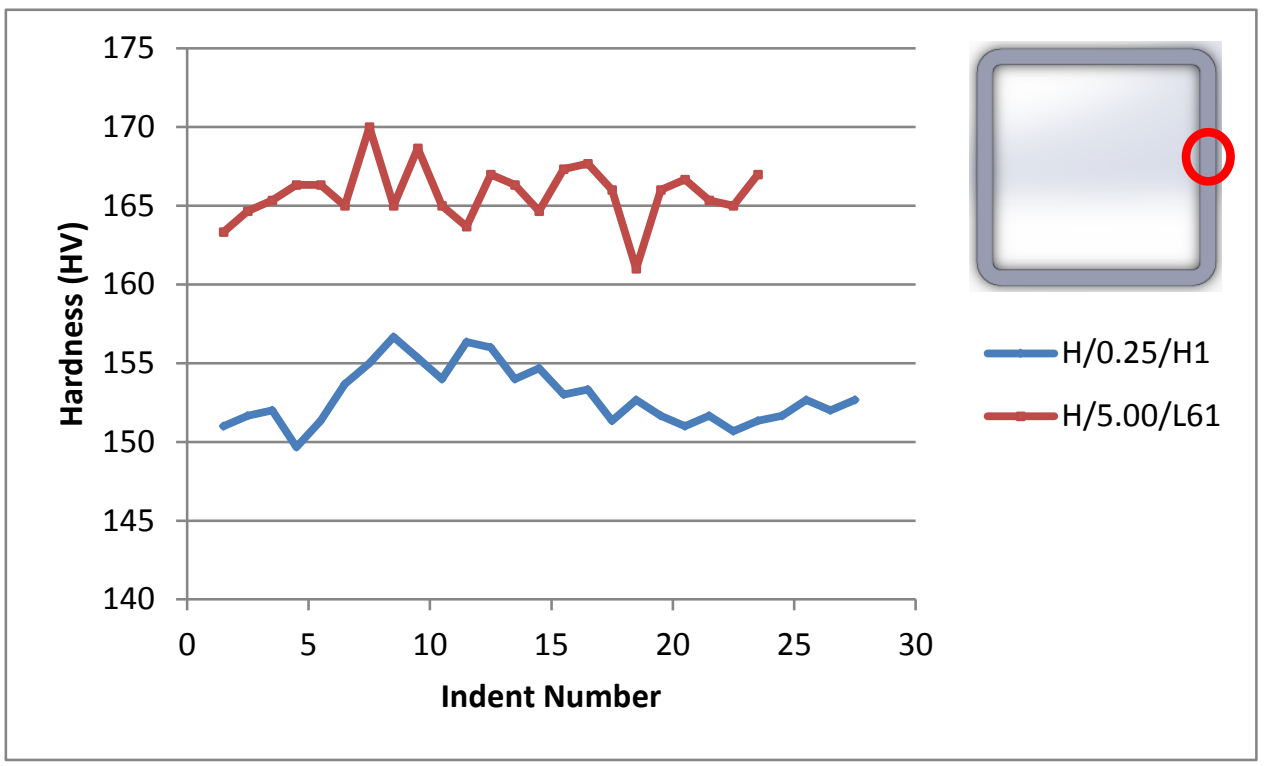

Figure 54-Graph plotting high against low strain, hot-finished RHS crosssectional hardness at the intrados surface

It can be seen in Figure 54 that bending the sample increases the hardness at the intrados cross section from approximately 152 to $167 \mathrm{HV}$. This is a similar increase than the hardness increase experienced at the intrados surface. However, the absolute hardness of the surface is greater than that of the cross section. 


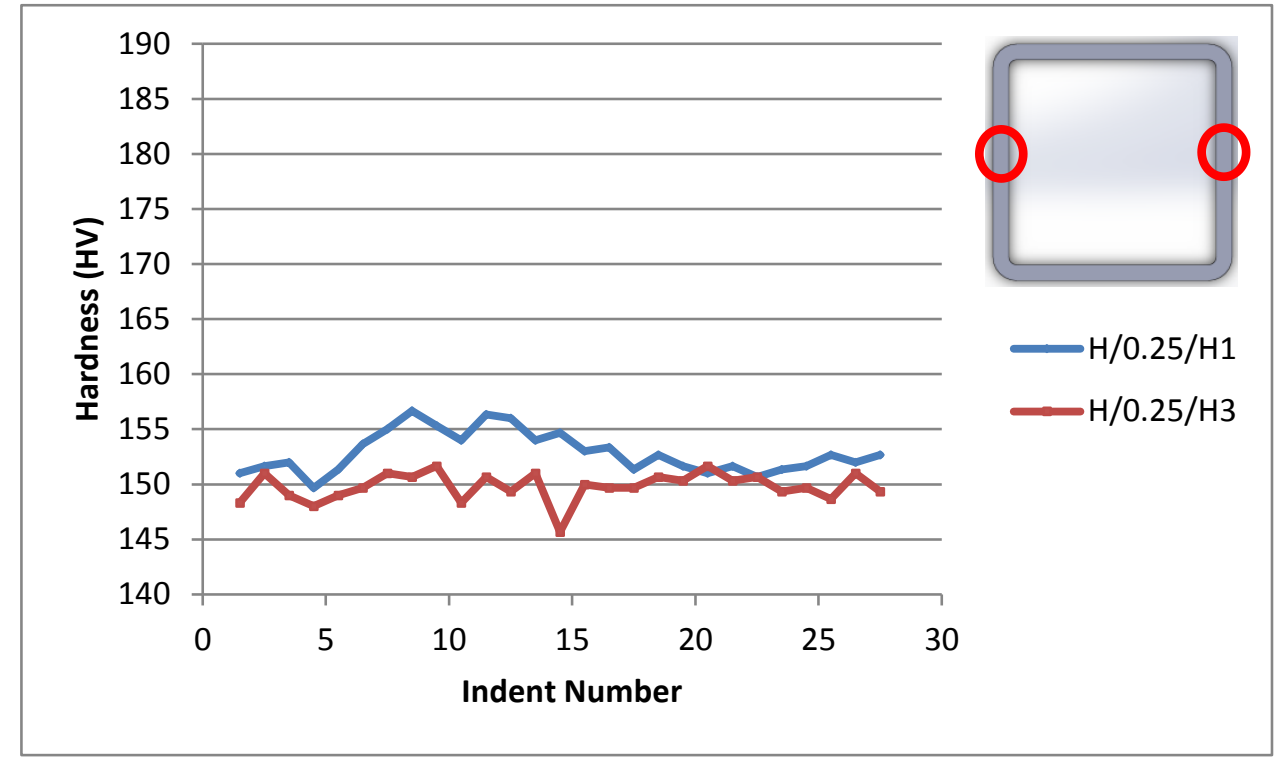

Figure 55-Graph plotting low strain, hot-finished RHS cross-sectional hardness at the intrados against the extrados surface

Figure 55 plots the variation in low strain cross sectional hardness, comparing the intrados to the extrados. The intrados has a harder cross section with a mean hardness of 3 Vickers more than the extrados, as observed from the surface testing.

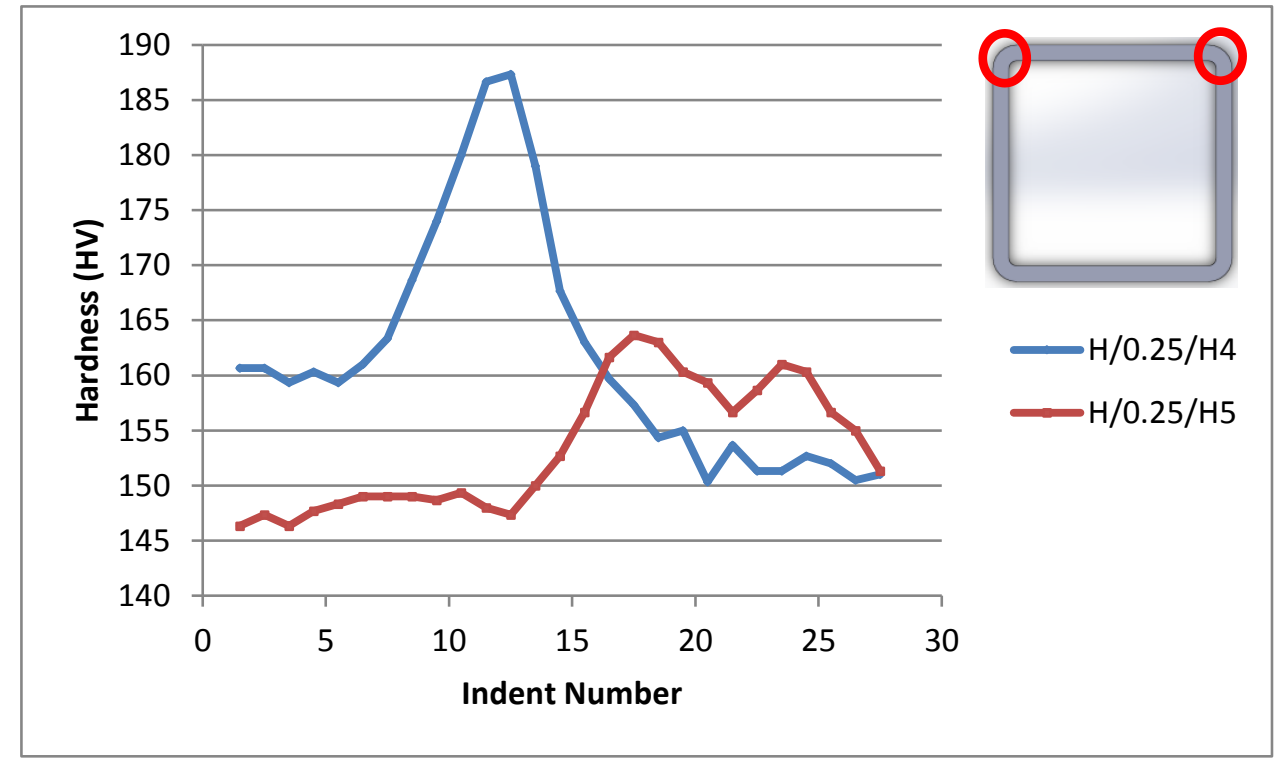

Figure 56-Graph plotting hot-finished RHS cross sectional hardness at the intrados and extrados low strain samples. 
Figure 56 plots the variation in cross sectional hardness for the low strain, hot-finished RHS at the intrados and extrados corners. As with the intrados and extrados flat sample areas, the intrados corner area is harder than extrados corner region. A relatively large increase in hardness can be seen

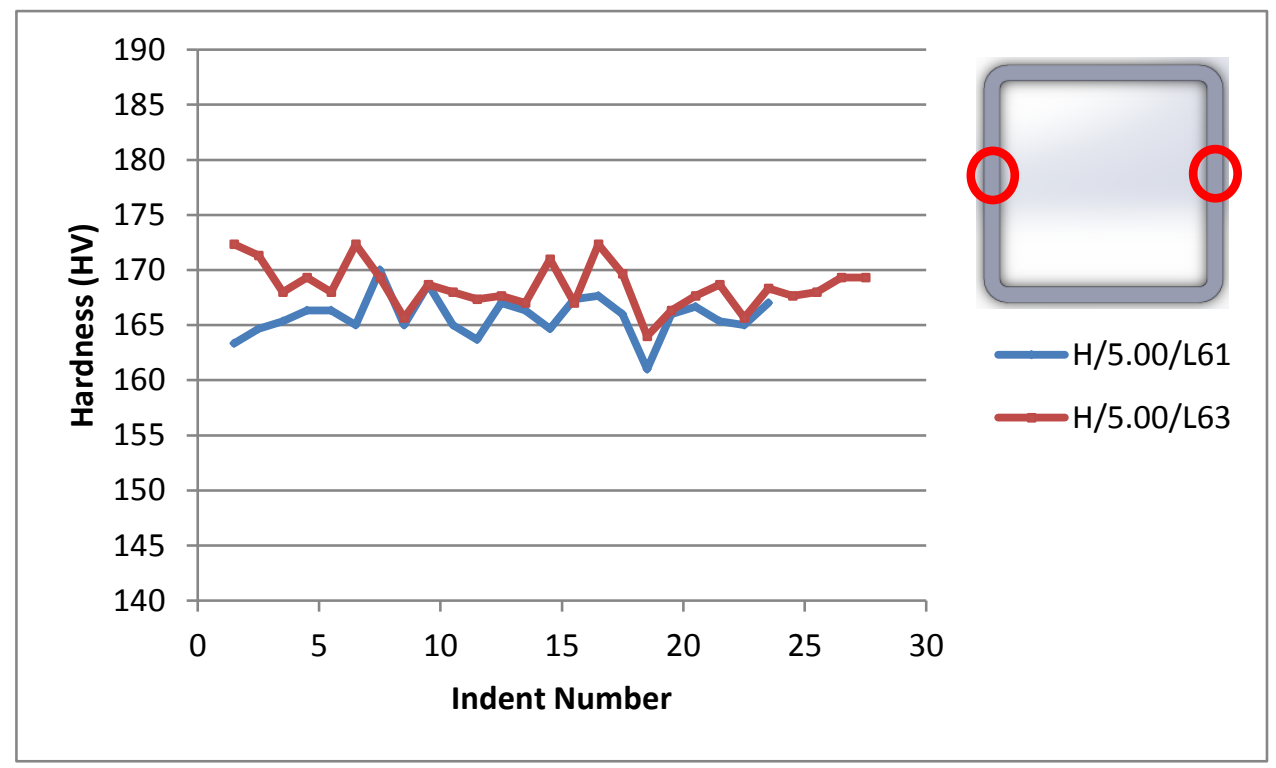

Figure 57-Graph plotting high strain, hot-finished RHS cross-sectional hardness at the intrados against the extrados

Figure 57 plots the variation in hot-finished RHS cross sectional hardness at the intrados and extrados. The extrados has a mean hardness that is 2 Vickers greater than the intrados in this sample. 


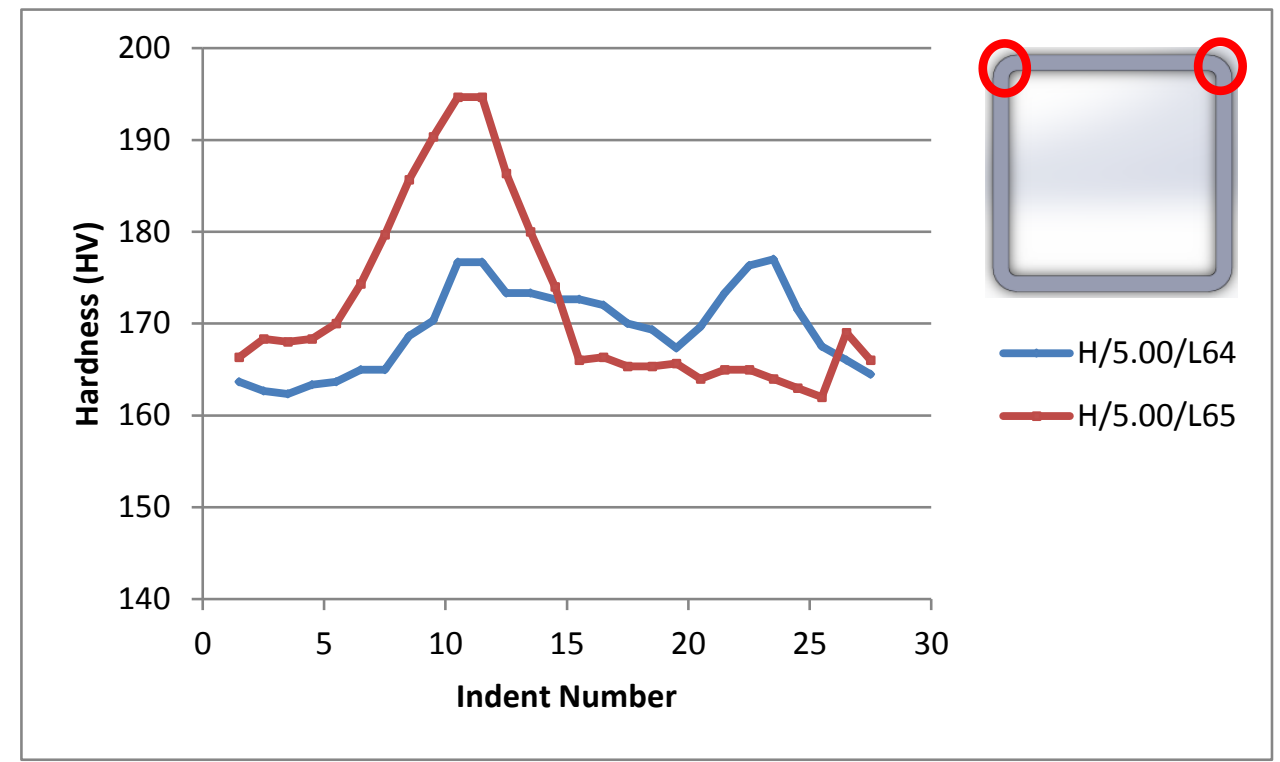

Figure 58-Graph plotting high strain, hot-finished RHS cross-sectional hardness at the intrados corner against the extrados corner.

Figure 58 plots the high strain, hot-finished RHS cross-sectional hardness at the intrados and extrados corners.

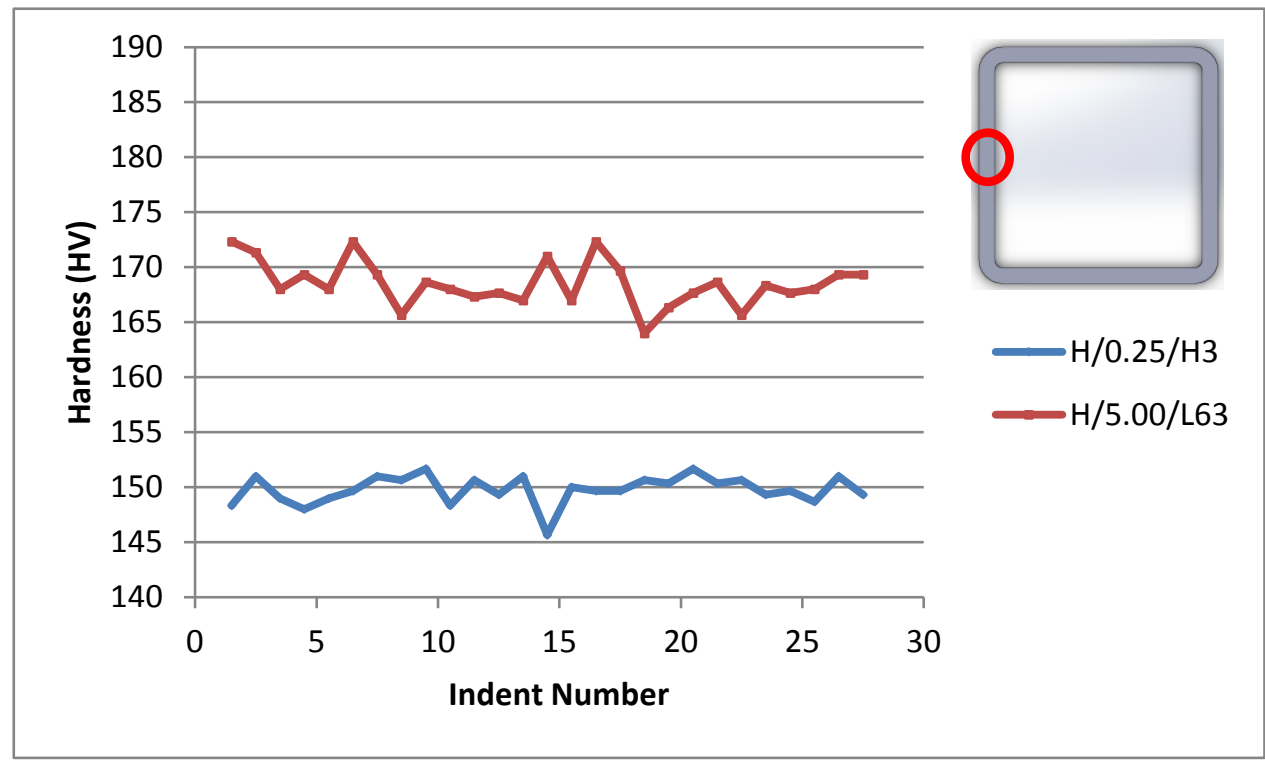

Figure 59-Graph plotting hot-finished RHS cross sectional hardness at the extrados of both high and low strain samples.

Figure 59 plots the variation in cross-sectional hardness for the extrados area of the hot-finished RHS for the low and high strain rate samples. It is clear from the figure that the bending has increased the hardness of the region from an average of approximately $150 \mathrm{HV}$ at $0.25 \%$ strain to above $165 \mathrm{HV}$ at $5 \%$ stain. 


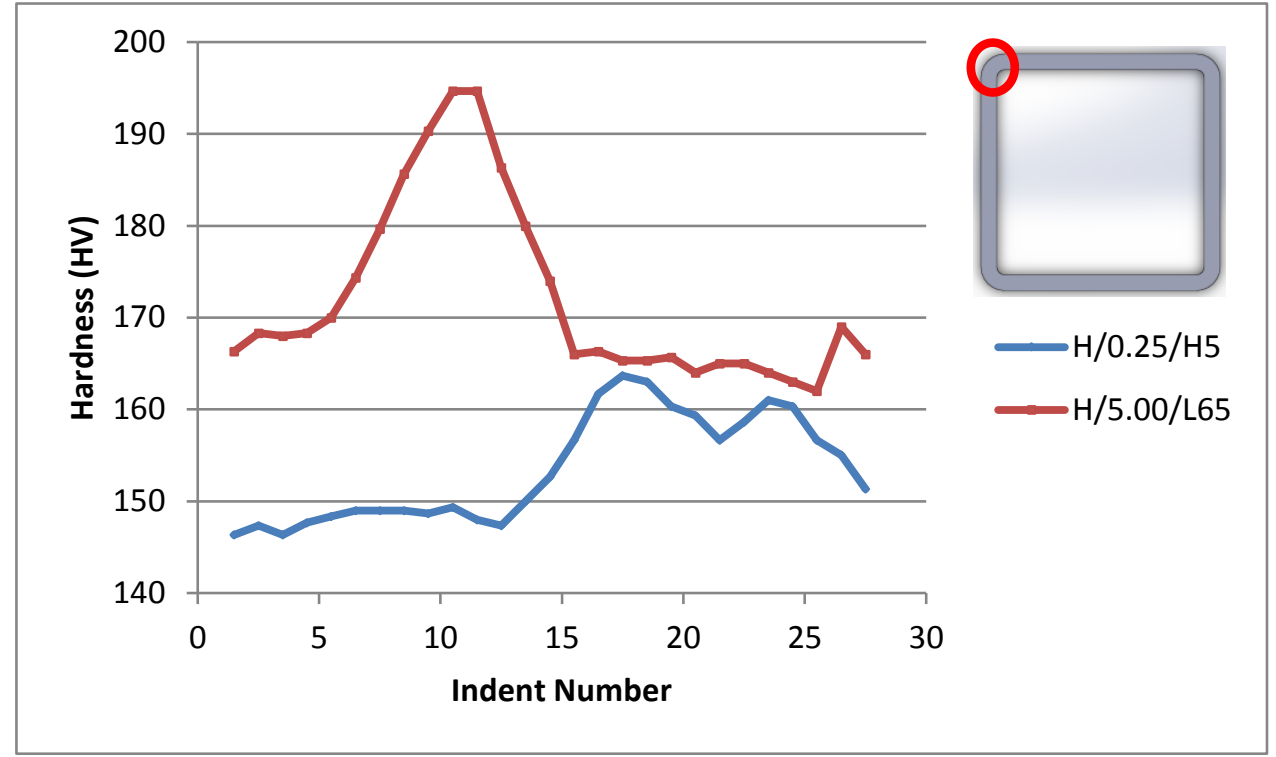

Figure 60-Graph plotting hot-finished RHS cross sectional hardness at the extrados corner of both high and low strain samples.

Figure 60 plots the variation in extrados corner cross-sectional hardness for the hot-finished RHS samples at low and high strain rates. It is clear from the figure that the bending has increased the hardness of the corner region from a maximum of $164 \mathrm{HV}$ at $0.25 \%$ strain to above $195 \mathrm{HV}$ at $5 \%$ stain. However, it is clear that not the entire sample has experienced the same increase in hardness as only as small area experiences significant alteration in profile. This increase is brought about when the sample is pressed into the forming rolls during bending, tightening the corer radii. A relatively small region is affected by this process. 


\subsubsection{Cold-formed Square Hollow Section}

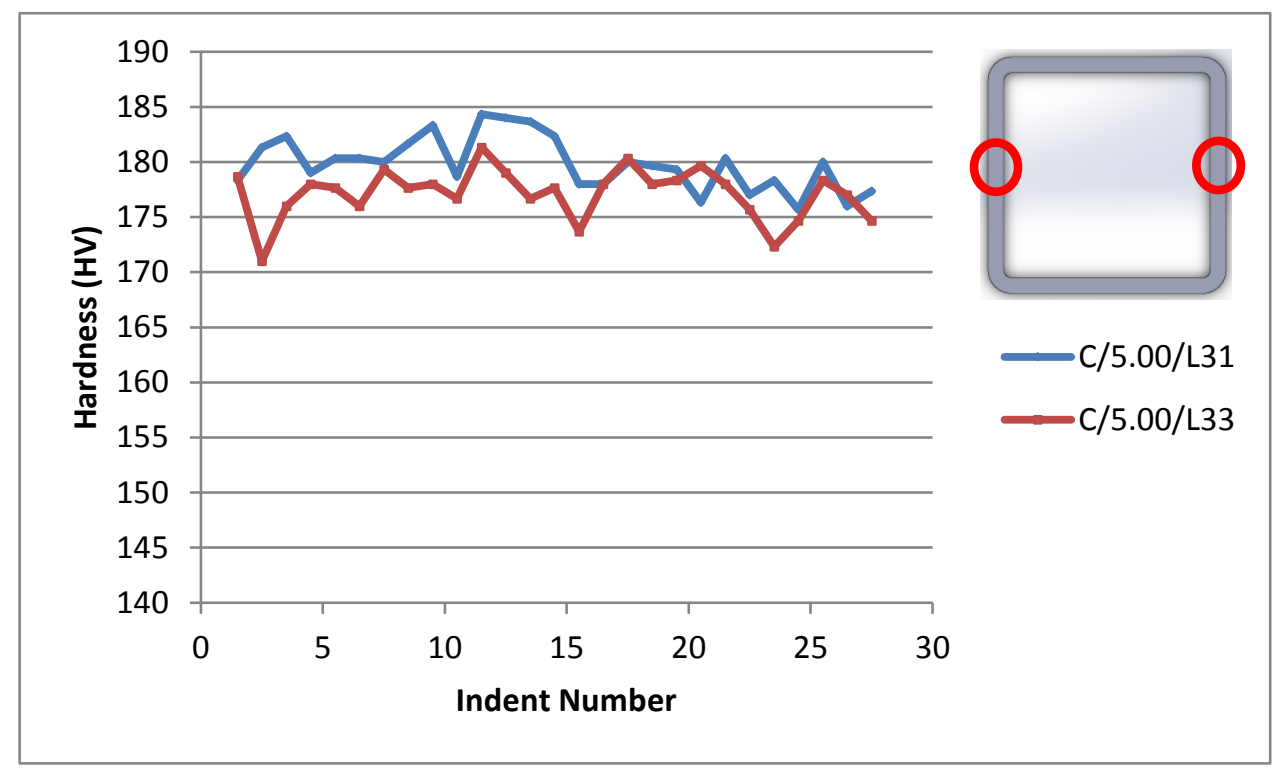

Figure 61-Graph plotting high strain, cold-formed RHS cross-sectional hardness at the intrados against the extrados.

Figure 61 plots the cross-sectional hardness at both the intrados and extrados for the cold-formed RHS samples after bending to a high strain rate. The average hardness of the extrados is 3 Vickers lower than that of the intrados which could be considered to be within measurement error of the equipment. 


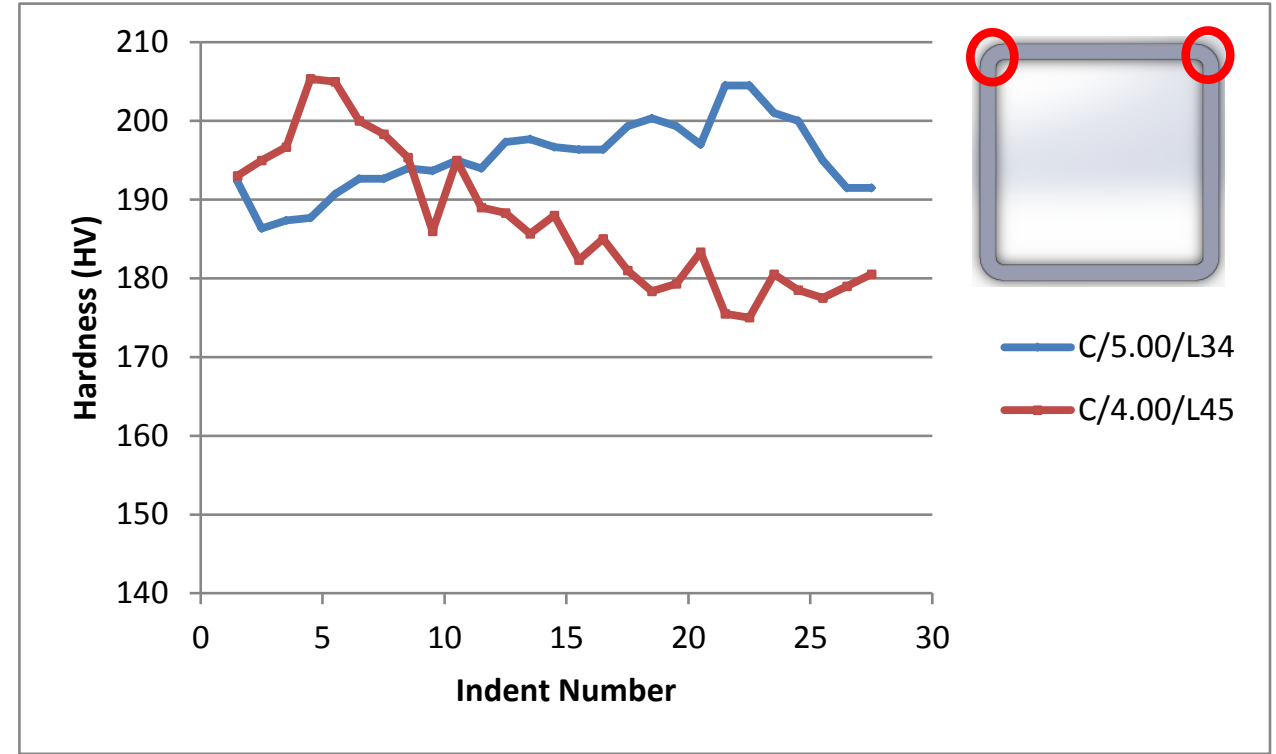

Figure 62-Graph plotting high strain cold-formed RHS cross-sectional hardness at the intrados corner against the extrados corner.

Figure 62 plots the cross-sectional hardness at both the intrados and extrados corners for the coldformed rectangular hollow section samples after bending to a high strain rate. The mean hardness of the extrados is marginally lower than that of the intrados. 


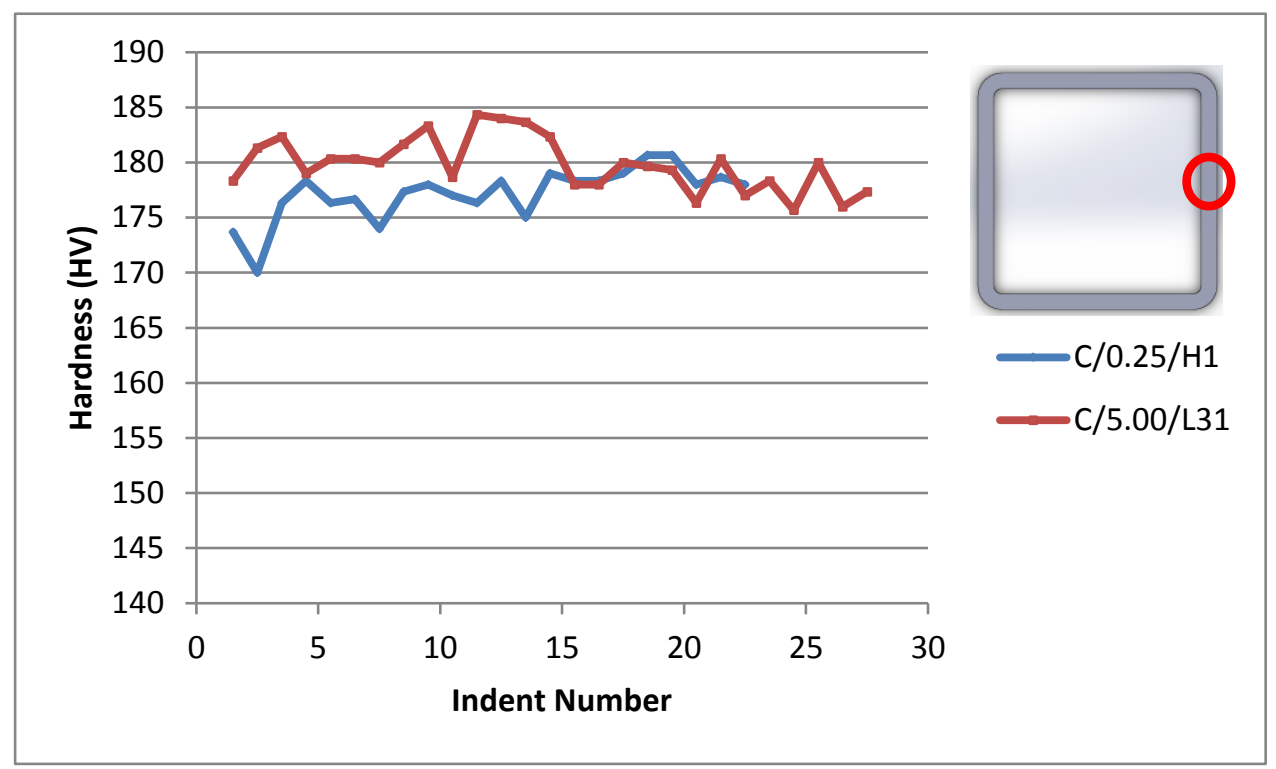

Figure 63-Graph plotting, cold-formed RHS cross-sectional hardness at the intrados for both low and high strain samples.

Figure 63 plots the cross-sectional hardness at the intrados for the cold-formed rectangular hollow section samples at low and high strain rates. Very little increase in hardness is observed from the additional bending from $0.25 \%$ to $5 \%$ at the intrados. 


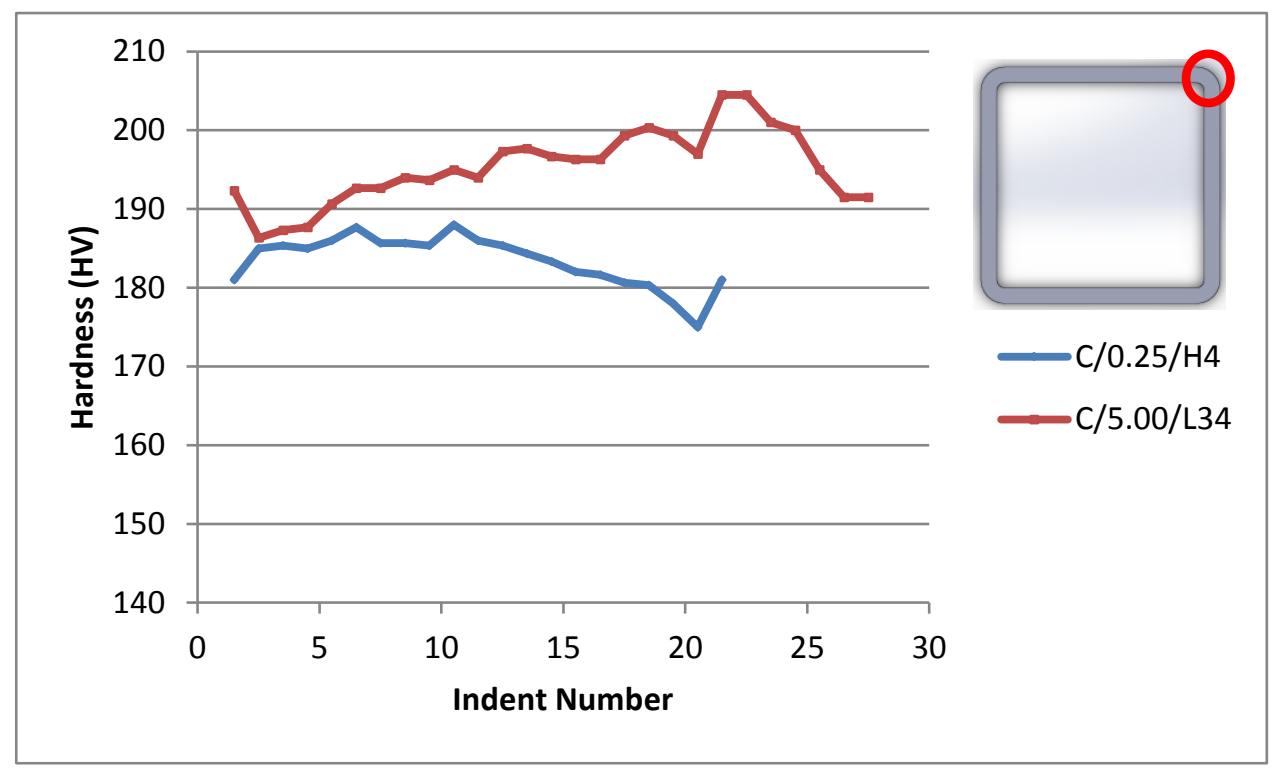

Figure 64-Graph plotting, cold-formed RHS cross-sectional hardness at the intrados corner for both low and high strain samples.

Figure 64 plots the cross-sectional hardness at the intrados corner for the cold-formed rectangular hollow section samples at low and high strain rates. It is clear from the above figure that the bending has increased the hardness of the corner region from a maximum of $189 \mathrm{HV}$ at $0.25 \%$ strain to above $200 \mathrm{HV}$ at $5 \%$ stain. However, it is clear that only a relatively small area of the sample has experienced an increase in cross-sectional hardness. This increase is brought about when the sample is pressed into the forming rolls during bending, tightening the corer radii. A relatively small region is affected by this process. 


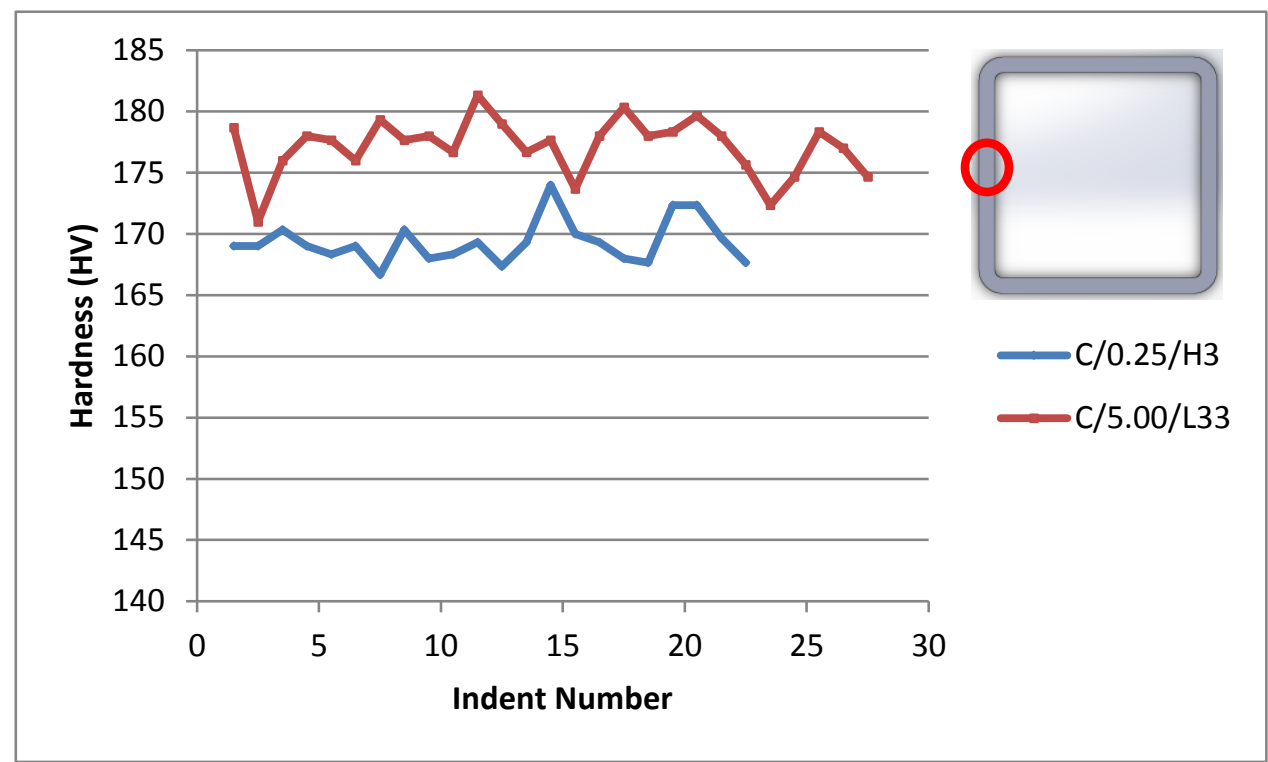

Figure 65-Graph plotting, cold-formed RHS cross-sectional hardness at the extrados for both low and high strain samples.

Figure 65 plots the cross-sectional hardness at the extrados for the cold-formed rectangular hollow section samples at low and high strain rates. It is clear from the above figure that the bending has increased the mean hardness of the extrados from approximately $170 \mathrm{HV}$ at $0.25 \%$ strain to approximately $177 \mathrm{HV}$ at $5 \%$ stain. This is a slightly greater increase than is observed at the intrados. 


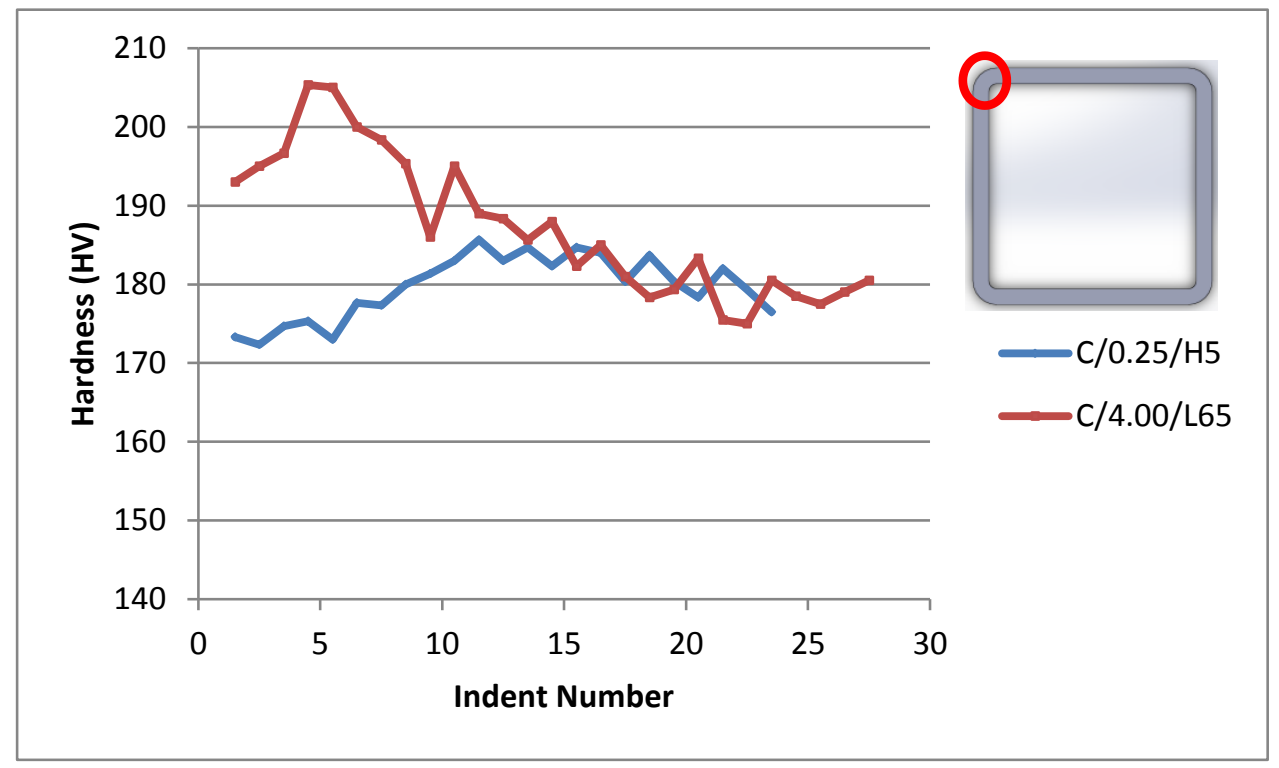

Figure 66-Graph plotting, cold-formed RHS cross-sectional hardness at the intrados corner for both low and high strain samples.

Figure 66 plots the cross-sectional hardness for at the extrados corner of the cold-formed rectangular hollow section samples at low and high strain rates. It is clear from the figure that the bending has increased the hardness of the corner region from a maximum of $185 \mathrm{HV}$ at $0.25 \%$ strain to above $200 \mathrm{HV}$ at $4 \%$ stain. However, it is clear that not the entire sample has experienced an increase in hardness as only as small area experiences significant alteration in profile. However, it is clear that only a relatively small area of the sample has experienced an increase in cross-sectional hardness. This increase is brought about when the sample is pressed into the forming rolls during bending, tightening the corer radii. A relatively small region is affected by this process. 


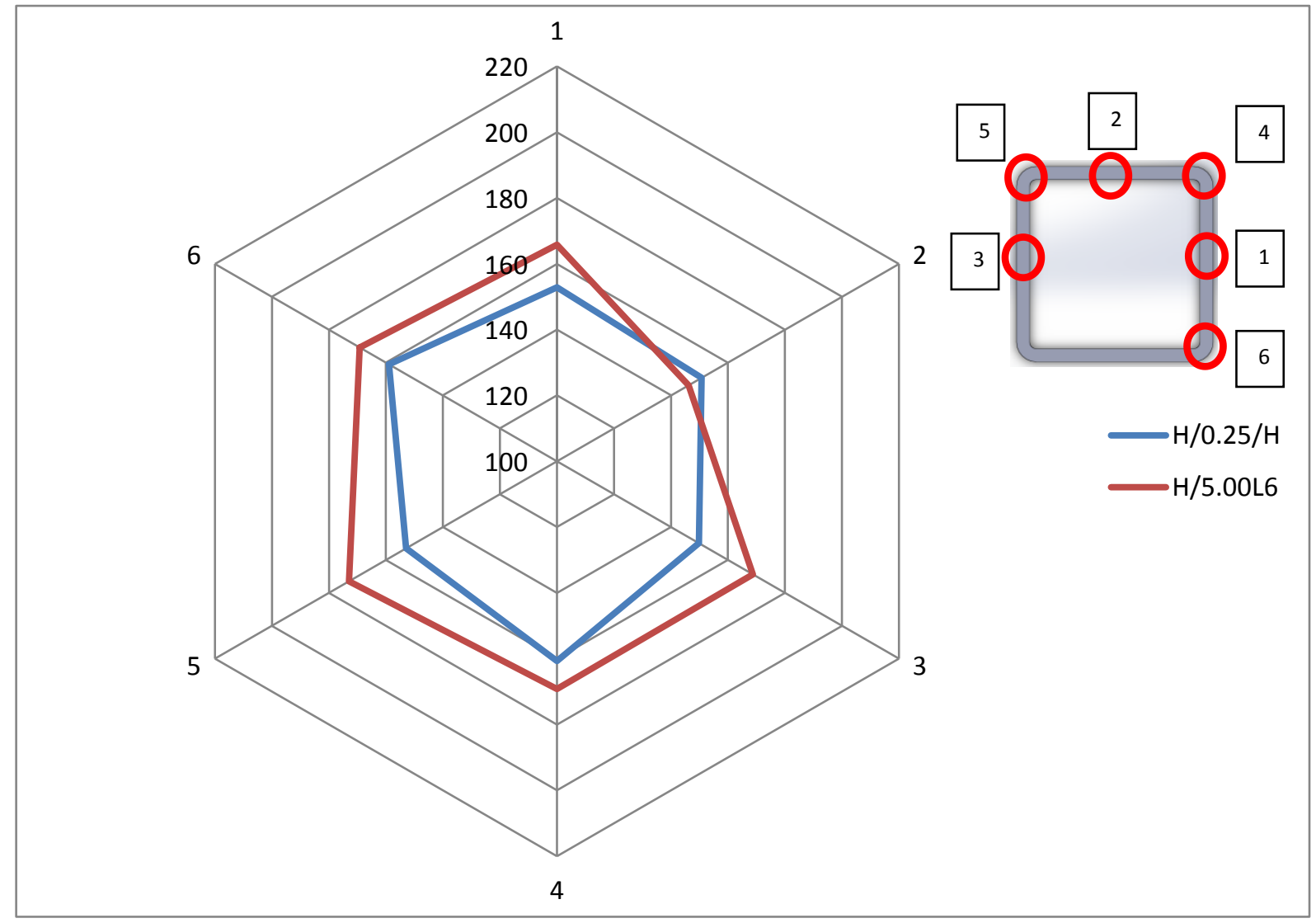

Figure 67-Mean cross section Vickers hardness for the testing areas of the hot-finished RHS

Figure 67 plots the mean cross-sectional hardness of the TATA Steel hot-finished RHS at each of the testing areas. The blue line shows the low strain hardness values and the red line shows the high strain hardness values. A significant increase in average hardness at sample positions 1, 3, 4,5 and 6 (the intrados face, the extrados face, the intrados corner, the extrados corner and the intrados corner weld area respectively) can be seen. In all of these areas the hardness increased from approximately 155 to $170 \mathrm{HV}$. However, at position 2 (the neutral axis) the hardness has remained largely unchanged. The most notable change in hardness was experienced at the extrados corner (position 5). The most likely reason for this greater increase in hardness is that this region is subjected to the highest tension during the cold bending process. The base hardness of the unbent hot finished RHS is $150 \mathrm{HV}$, the hardness of the intrados corners increased to 160 after cold bending to $0.25 \%$ strain. Bending to $0.25 \%$ only marginally affected the hardness of the other areas. 


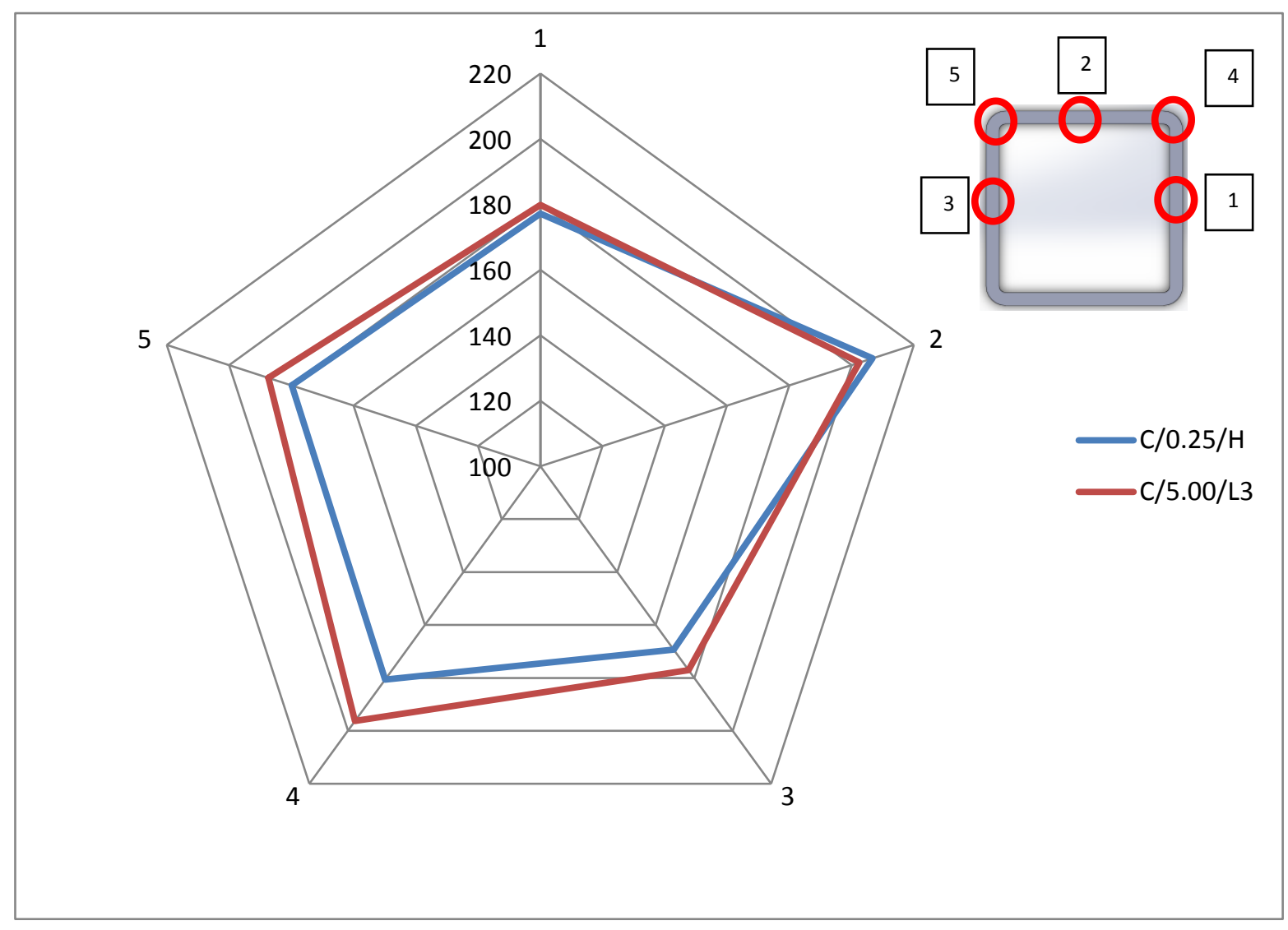

Figure 68-Mean cross section Vickers hardness for the testing areas of the cold-formed RHS

Figure 68 shows the change in cross-sectional hardness of the cold formed RHS at each sample location due to the cold bending process. It can be seen that there was little change in average hardness at sample positions 1 and 2 (the intrados and neutral axis respectively). However, there was an increase in hardness at locations 3, 4 and 5 (the intrados corner, extrados face and extrados corner respectively). The largest increase in hardness was observed at the intrados corner (position 4), the position subjected to the greatest degree of multi-directional stress. An increase in hardness was expected for the extrados face and corner (position 4 and 5 respectively) as these positions were under tension. As for the hot-finished RHS, position 2 (the neutral axis face) showed no significant change in hardness as the areas does not experience any significant deformation or stress. 


\subsection{Tensile Testing}

Tensile testing is a fundamental method in which a sample is subjected controlled tension until failure. The results from the test are commonly used to select a material for an application, quality control, or to predict how a material will react under other types of forces. Properties that are directly measured during a tensile test are yield strength, ultimate tensile strength and maximum elongation. The reduction in area, caused by necking is recorded after the test is complete. From these measurements, a number of other properties of the material can be calculated including the: Young's Modulus, Poisson's ratio and the strain-hardening characteristics. The results are usually plotted in a graph of stress against strain. Uniaxial tensile testing is the most commonly used for obtaining the mechanical characteristics of isotropic materials. Due to the grain orientation caused during production of the tubes and subsequent bending, the tensile strength of the steel is not isotropic. 
6.3.1. Hot-finished Rectangular Hollow Section

\begin{tabular}{|c|c|c|c|c|c|c|c|c|}
\hline Sample Ref & Apparatus & Position & $\begin{array}{c}\text { Thickness } \\
\text { (mm) }\end{array}$ & $\begin{array}{l}\text { Width } \\
\text { (mm) }\end{array}$ & $\begin{array}{l}\text { Yield } \\
\text { Stress } \\
\text { (MPa) }\end{array}$ & $\begin{array}{l}\text { Proof } \\
0.5 \% \\
\text { (MPa) }\end{array}$ & $\begin{array}{l}\text { Proof } \\
0.2 \% \\
\text { (MPa) }\end{array}$ & $\begin{array}{l}\text { UT: } \\
\text { (MP }\end{array}$ \\
\hline$H / 0.00 / H$ & Schenck 250kN & Intrados & 9.4 & 19.93 & 377 & 373 & 374 & 505 \\
\hline$H / 0.00 / H$ & Schenck 250kN & Extrados & 9.41 & 20.17 & 370 & 370 & 370 & 524 \\
\hline $\mathrm{H} / 0.50 / \mathrm{H}$ & Schenck 250kN & Intrados & 9.32 & 19.91 & - & 340 & 324 & 512 \\
\hline$H / 0.50 / H$ & Schenck 250kN & Extrados & 20.22 & 9.29 & - & 366 & 363 & 508 \\
\hline$H / 1.00 / H$ & Schenck 250kN & Intrados & 9.42 & 19.88 & - & 321 & 300 & 509 \\
\hline$H / 1.00 / H$ & Schenck 250kN & Extrados & 9.38 & 20.28 & - & 359 & 356 & 507 \\
\hline $\mathrm{H} / 2.00 / \mathrm{H}$ & Schenck 250kN & Intrados & 9.42 & 19.88 & - & 296 & 274 & 510 \\
\hline$H / 2.00 / H$ & Schenck 250kN & Extrados & 9.27 & 20.13 & - & 364 & 363 & 516 \\
\hline $\mathrm{H} / 4.00 / \mathrm{H}$ & Schenck 250kN & Intrados & 9.69 & 19.87 & - & 331 & 315 & 510 \\
\hline$H / 4.00 / H$ & Schenck 250kN & Extrados & 9.18 & 20.18 & - & 410 & 399 & 524 \\
\hline H/4.00/L6 & Schenck 250kN & Intrados & 9.64 & 19.91 & - & 318 & 297 & 508 \\
\hline H/4.00/L6 & Schenck 250kN & Extrados & 9.22 & 20.26 & - & 397 & 389 & 518 \\
\hline $\mathrm{H} / 5.00 / \mathrm{H}$ & Schenck 250kN & Intrados & 9.74 & 19.84 & - & 323 & 308 & 504 \\
\hline $\mathrm{H} / 5.00 / \mathrm{H}$ & Schenck 250kN & Extrados & 9.26 & 20.28 & - & 414 & 406 & 523 \\
\hline
\end{tabular}

Table 6-Hot-finished RHS tensile strength at the intrados and extrados where the figures labelled in red are $355 \mathrm{MPa}$ 


\begin{tabular}{|c|c|c|c|c|c|c|c|c|}
\hline Sample Ref & Apparatus & Position & $\begin{array}{l}\text { Thickness } \\
\text { (mm) }\end{array}$ & $\begin{array}{l}\text { Width } \\
\text { (mm) }\end{array}$ & $\begin{array}{l}\text { Area } \\
\left(\mathrm{mm}^{2}\right)\end{array}$ & $\begin{array}{l}\text { Yield } \\
\text { Stress } \\
\text { (MPa) }\end{array}$ & $\begin{array}{l}\text { Proof } \\
0.5 \% \\
\text { (MPa) }\end{array}$ & $\begin{array}{c}\text { Proof } \\
0.2 \% \\
\text { (MPa) }\end{array}$ \\
\hline$H / 0.00 / H$ & Mayes 500KN & Extrados & 9.45 & 20.09 & 189.9 & 378 & 364 & 365 \\
\hline$H / 0.00 / H$ & Mayes 500KN & Intrados & 9.38 & 20.15 & 189 & 410 & 392 & 379 \\
\hline$H / 0.25 / H$ & Mayes 500KN & Extrados & 9.31 & 20.07 & 186.9 & - & 415 & 392 \\
\hline$H / 0.25 / H$ & Mayes 500KN & Intrados & 9.31 & 20.13 & 187.4 & 370 & 369 & 363 \\
\hline$H / 0.50 / H$ & Schenck 250KN & Extrados & 9.23 & 20.22 & 187.8 & - & 366 & 363 \\
\hline$H / 0.50 / H$ & Schenck 250KN & Intrados & 9.34 & 20.13 & 188 & - & 327 & 313 \\
\hline$H / 1.00 / H$ & Schenck 250KN & Extrados & 9.3 & 20.06 & 186.6 & - & 365 & 363 \\
\hline$H / 1.00 / H$ & Schenck 250KN & Intrados & 9.38 & 20.11 & 188.6 & - & 318 & 293 \\
\hline$H / 2.00 / H$ & Schenck 250KN & Extrados & 9.27 & 20.13 & 186.6 & - & 364 & 363 \\
\hline$H / 2.00 / H$ & Schenck 250KN & Intrados & 9.5 & 20.10 & 190.9 & - & 280 & 268 \\
\hline$H / 3.00 / H$ & Schenck 250KN & Intrados & 9.49 & 20.06 & 190.4 & - & 297 & 284 \\
\hline$H / 4.00 / H$ & Mayes 500KN & Extrados & 9.19 & 20.10 & 184.7 & - & 447 & 429 \\
\hline$H / 4.00 / H$ & Mayes 500KN & Intrados & 9.57 & 20.12 & 192.6 & - & 451 & 441 \\
\hline $\mathrm{H} / 4.00 / \mathrm{H}$ & Mayes 500KN & Extrados & & & & - & 383 & 377 \\
\hline$H / 4.00 / L 6$ & Schenck 250KN & Intrados & 9.60 & 20.09 & 192.9 & - & 334 & 318 \\
\hline$H / 4.00 / L 6$ & Schenck 250KN & Intrados & 9.56 & 20.06 & 191.8 & - & 308 & 292 \\
\hline
\end{tabular}

Table 7-Tensile testing values recorded for the hot-finished rectangular hollow sections at the intrados and $\mathrm{e}$ in red are values lower than the standard $355 \mathrm{MPa}$ 
The standard testing procedures for curved steel include extracting samples from the neutral axis of the bend. There are a number of benefits to this including a simplified extraction of the sample and standard tensile testing procedure. A neutral axis of a RHS is flat, with no curvature. This makes sample preparation and testing easy as the sample has not undergone any significant deformation. A curved sample taken from the intrados or extrados requires straightening before testing. This procedure will affect the results obtained by the testing. The neutral axis of the curved section undergoes no net change in length and in some cases there is a large area around the neutral axis which has not been plastically deformed. This means that tensile testing at the neutral axis does not give an accurate depiction of the effect of bending on the mechanical properties of the section. It can be seen clearly from the above tables that the location from which a sample is extracted, affects the results that are collected. Before bending, all areas of the RHS showed similar proof stresses however after bending a large difference can be observed between different areas of the section. All intrados samples tested on the Schenck $250 \mathrm{kN}$ machine failed to meet the required specification of $355 \mathrm{MPa}$. The lowest recorded value was $268 \mathrm{MPa}$ a full $87 \mathrm{MPa}$ less than the required proof stress and $97 \mathrm{Mpa}$ less than the original, unbent material. Originally it was thought that the cause of this reduction of the proof stress was caused by the straightening process before bending. However, this cannot be the cause of the problem as the extrados does not exhibit the same phenomenon, despite undergoing the same straightening process.

It is likely that the reduction in proof stress observed has been caused by the Bauschinger effect. The Bauschinger effect is a phenomenon that occurs when plastic deformation has taken place. The deformation increases the yield strength in the direction of plastic flow and decreases it in the opposite direction. This means that areas deformed plastically in tension will have a reduced capacity in compression and areas in compression will have a reduced capacity in tension. Therefore the intrados of a bent section should have a reduced yield stress in tension when compared to the unbend material. This can be seen in Table 7-Tensile testing values recorded for the hot-finished rectangular hollow sections at the intrados and extrados. The same phenomenon occurs at the extrados, however; it has not observed during tensile testing. The reason for this is that the extrados is in tension during bending and then pulled further in tension by the tensile test. This will not show signs of the phenomenon as the reduced capacity will be in compression. To detect whether the phenomenon has been occurred, compression testing would be required. 
The significant majority of the curved samples tested do not have a distinct upper yield point. This is caused by the deformation of the material beyond the upper yield point into discontinuous yielding.

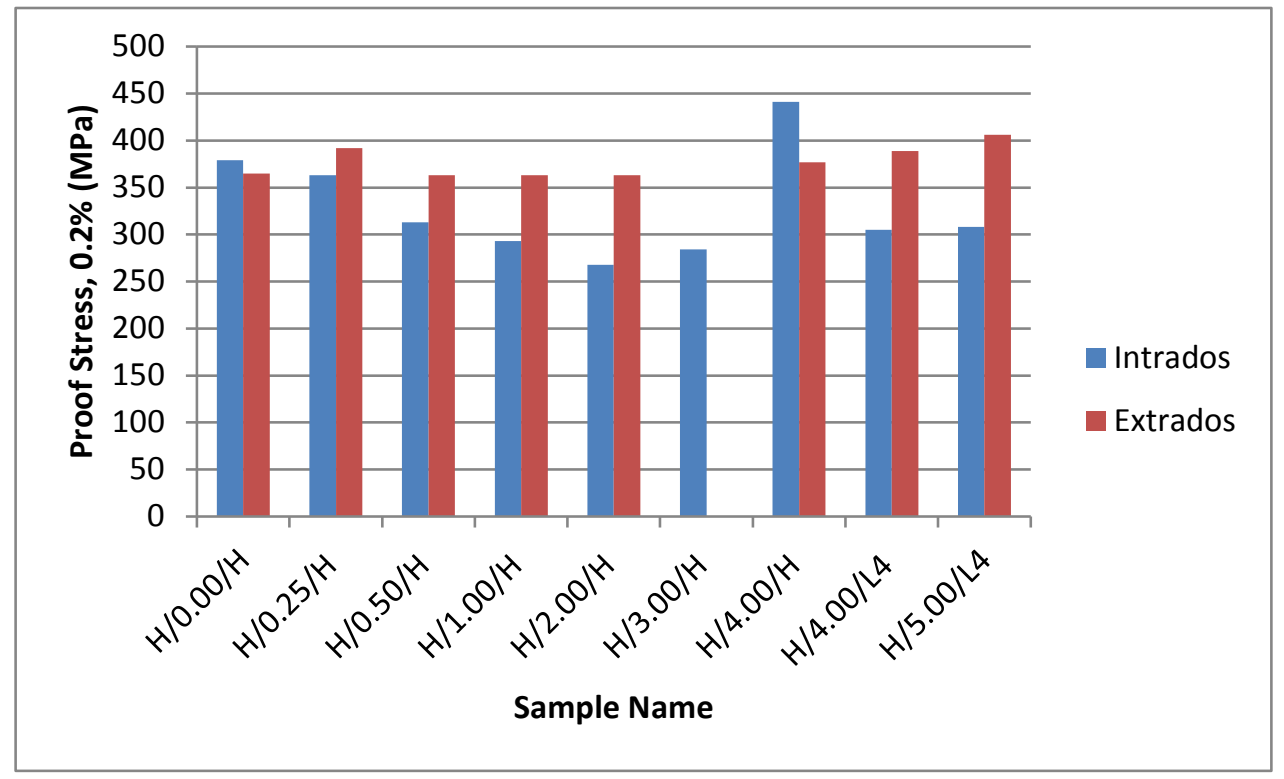

Figure 69-Graph plotting the difference in proof stress between the intrados and extrados of the hot-finished RHS with increasing strain

Once the steel has been stressed past its upper yield point into the discontinuous yielding zone, the feature will not be observed in a tensile test unless it has undergone a heat treatment prior to testing.

A steady decline in proof stress can be observed in Figure 69 for the intrados area samples until $2 \%$ strain. There is no clear increase in proof stress for the extrados within the same strain range. 


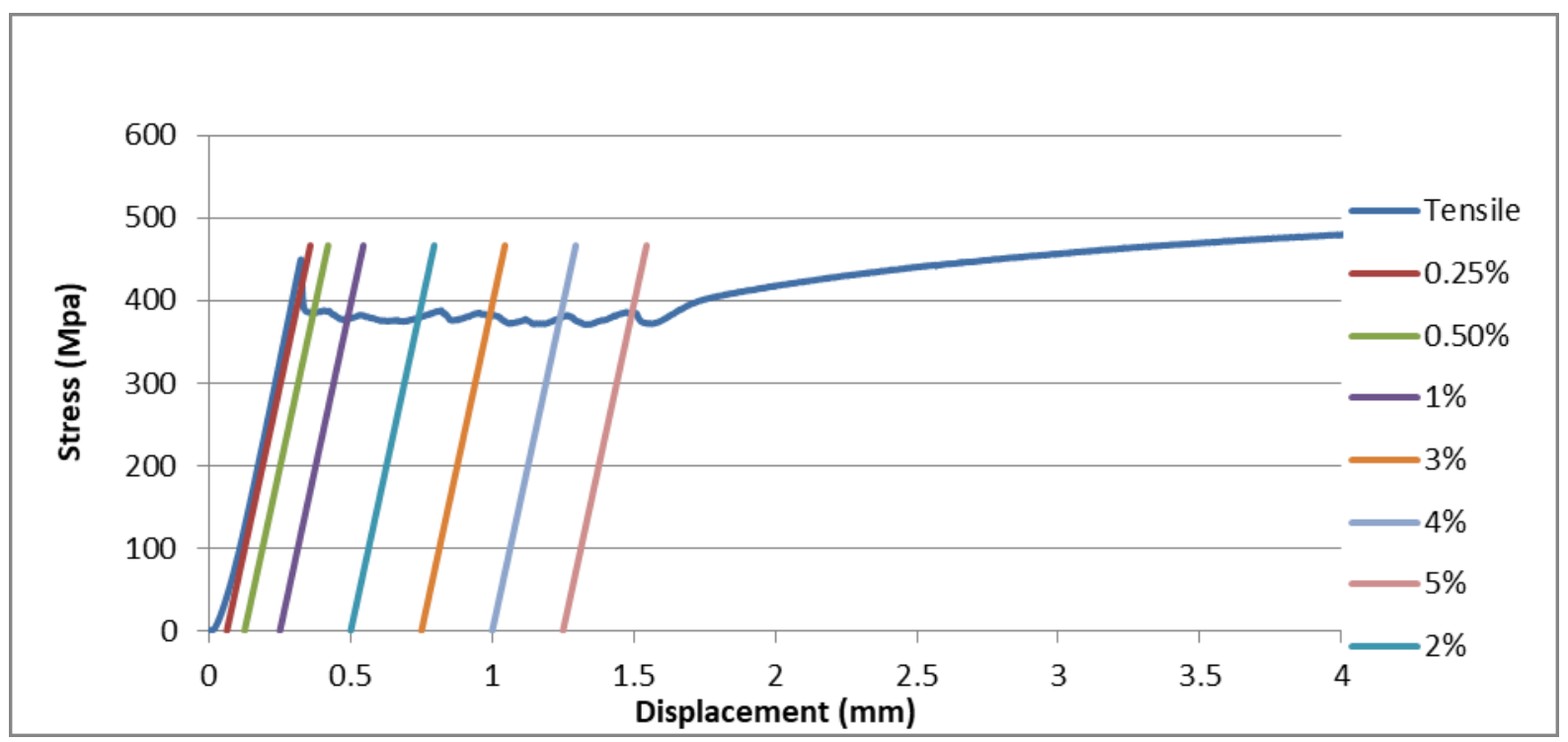

Figure 70-Graph plotting stress against displacement for hot-finished RHS

There is no trend that suggests that work hardening has taken place from Table 7-. This is likely due to the fact that the material has only been strained within the discontinuous yielding area of its stress-strain graph. Any subsequent strain would take the material past this point and initiate work hardening of the material. 


\subsubsection{Axial Reversed-displacement Testing}

Tension-compression and compression-tension tests have been carried out on hot-finished material to determine the influence of the Bauschinger effect on the material when axial loading is reversed. Reduction in yield stress of polycrystalline metals following plastic deformation in the opposite direction was reported by Bauschinger in 1881.[40], [57]

There are two theories that are thought to cause the Bauschinger effect; Orowan theory and back stress theory. During forward plastic deformation the moving dislocations interact with obstacles such as grain boundaries, precipitates or other dislocations. This prevents further movement and generates a back-stress around the interaction which resists further movement of the similarly signed dislocations. When the load is reversed, this back stress repels the dislocations in the opposite direction to the obstacle, away from the site of interaction. Therefore the stress field around the interaction site aids the movement of dislocations in the direction of the reverse strain, reducing the reverse yield stress proportionally to the quantity of back-stress. According to the back-stress theory, an increase in dislocation density increases the number of dislocation-dislocation interaction sites and therefore the back stress. Therefore the Bauschinger effect is greater in a material with high dislocation density. However, there is a theoretical maximum to the effect as an increase in dislocation density and pre-strain can lead to the number of mobile dislocations decreasing. This is caused by the immobilisation of dislocations at pile-ups as pre-strain is increased.

Alloying of a metal can also affect the phenomenon. Precipitated particles can act as interaction sites, further increasing the back-stress. However, not all particles contribute the dislocation pinning equally. The chemistry, size and distribution of the particles are important.

After detecting the Bauschinger effect at the intrados, further testing was carried out to assess whether it would occur at the extrados. This required carrying out compressive tests. A new test was devised that would simulate bending by pulling a test sample in tension to a set strain before compressing it and measuring its compressive strength. For this test a specialised test piece was produced in order to get accurate results. The test piece would have to be gripped so that it could be pulled for the tension phase but it would also need to undergo compressive stress so must be short and wide enough to avoid buckling. This was achieved by threading the ends of the test specimen. The ends were then threaded into specially designed collets which were clamped into the apparatus. Locking nuts were used to prevent the samples from becoming loose when the samples were elongated. 


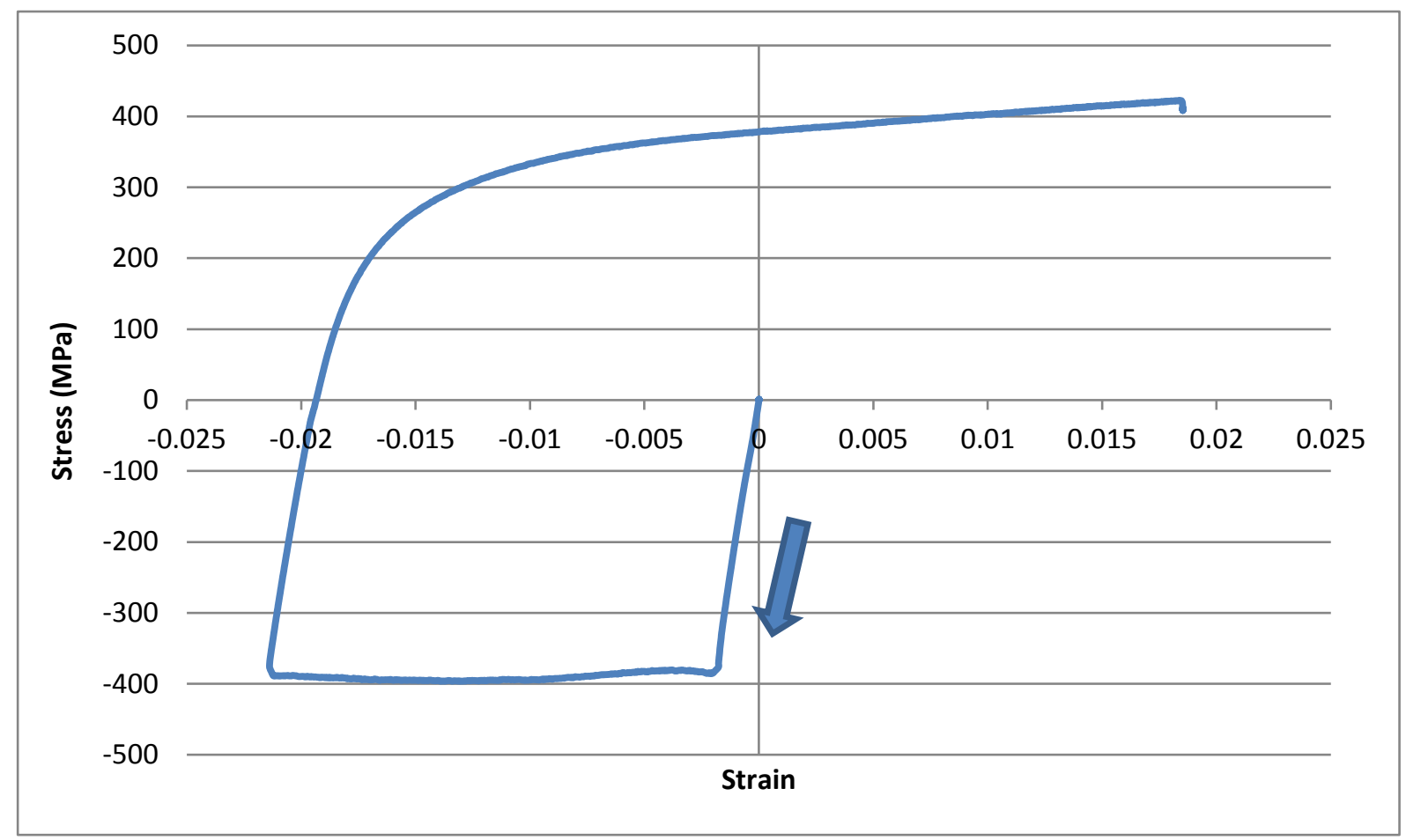

Figure 71-Graph plotting the stress against strain for a compression-tension test carried out on hot-finished material

Figure 71 plots the stress against strain for an axial compression-tension test carried out on hotfinished material. This test simulates the bending process at the intrados, (where the area experiences compressive stress), before tensile testing.

An upper yield point can be observed in the compression phase of the plot at approximately $370 \mathrm{MPa}$. The upper yield point feature is not present in the tension phase. It is clear that the sample has a reduced yield stress in tension after compression has taken place. The gradient of the stress strain curve in the tension phase of the test becomes non-linear at approximately $100 \mathrm{Mpa}$ which is significantly less than the specification requires (355MPa). This result is consistent with observations made during tensile testing of the intrados, where a significant reduction in proof stress was recorded. 


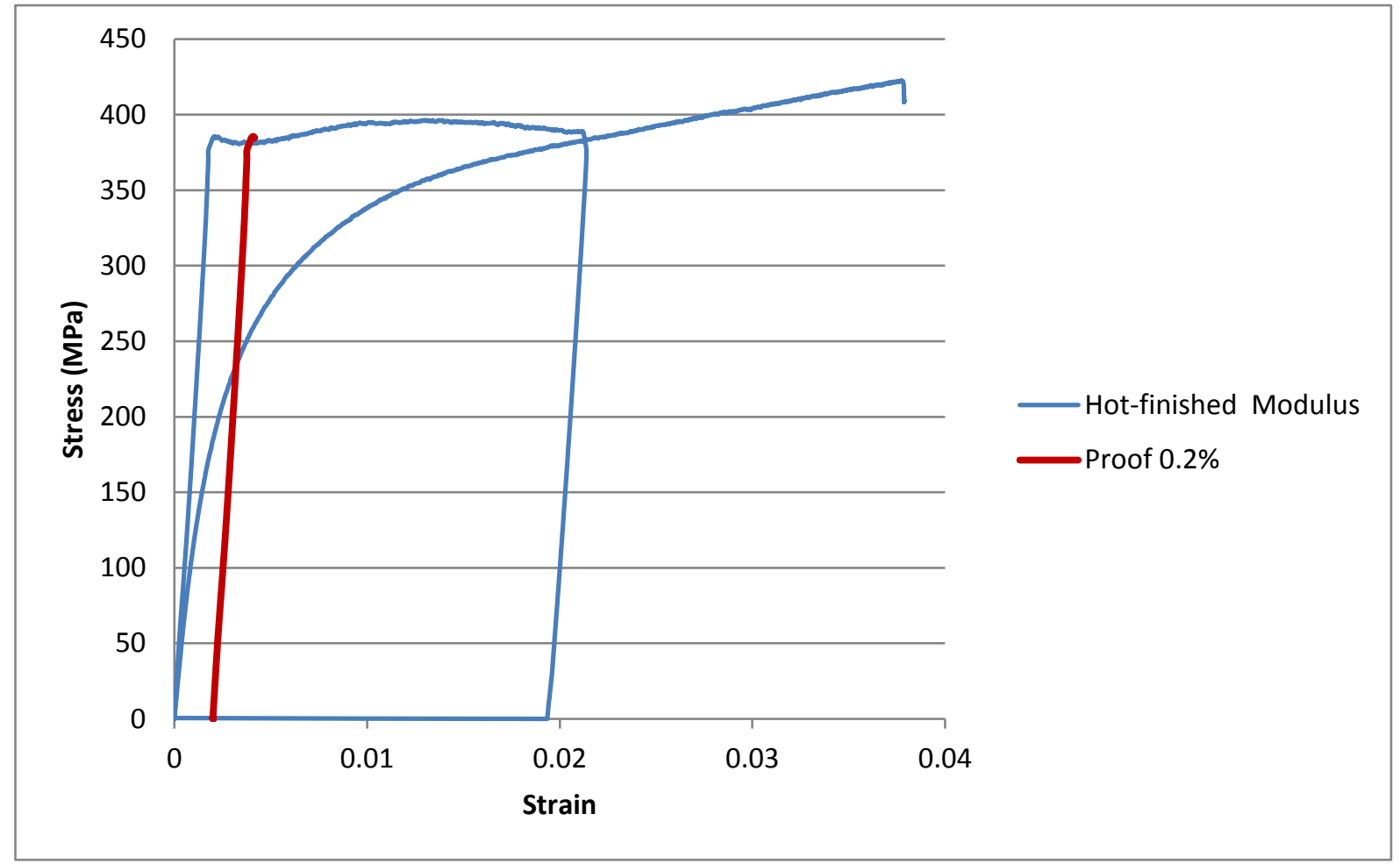

Figure 72-Graph plotting the modulus of stress against strain for a compression-tension test carried out on hot finished material, indicating the $0.2 \%$ proof stress

In Figure 72 both the compressive and tensile stress phases have been plotted on the same axis. The tension phase of the graph has been overlaid on the compression phase to depict the change in yield strength in tension after compression. It can be seen that there is a significant reduction in proof stress from above the $355 \mathrm{MPa}$ requirement in compression to approximately $240 \mathrm{MPa}$ in tension. However, it can be seen that the material quickly strain hardens and approximately achieves the same stress at 0.02 strain.

This testing has confirmed that the intrados area of the tube experiences a reduction in tensile yield strength, brought about by compressive strain during bending. Testing was then carried out to observe whether the same phenomenon occurs at the extrados where loading is reversed during the bending process. The testing was carried out at 2 different pre-strain values. 0.005 and 0.035 in order to determine whether there was a link between the magnitude of pre-strain and reduction in yield/proof stress. 


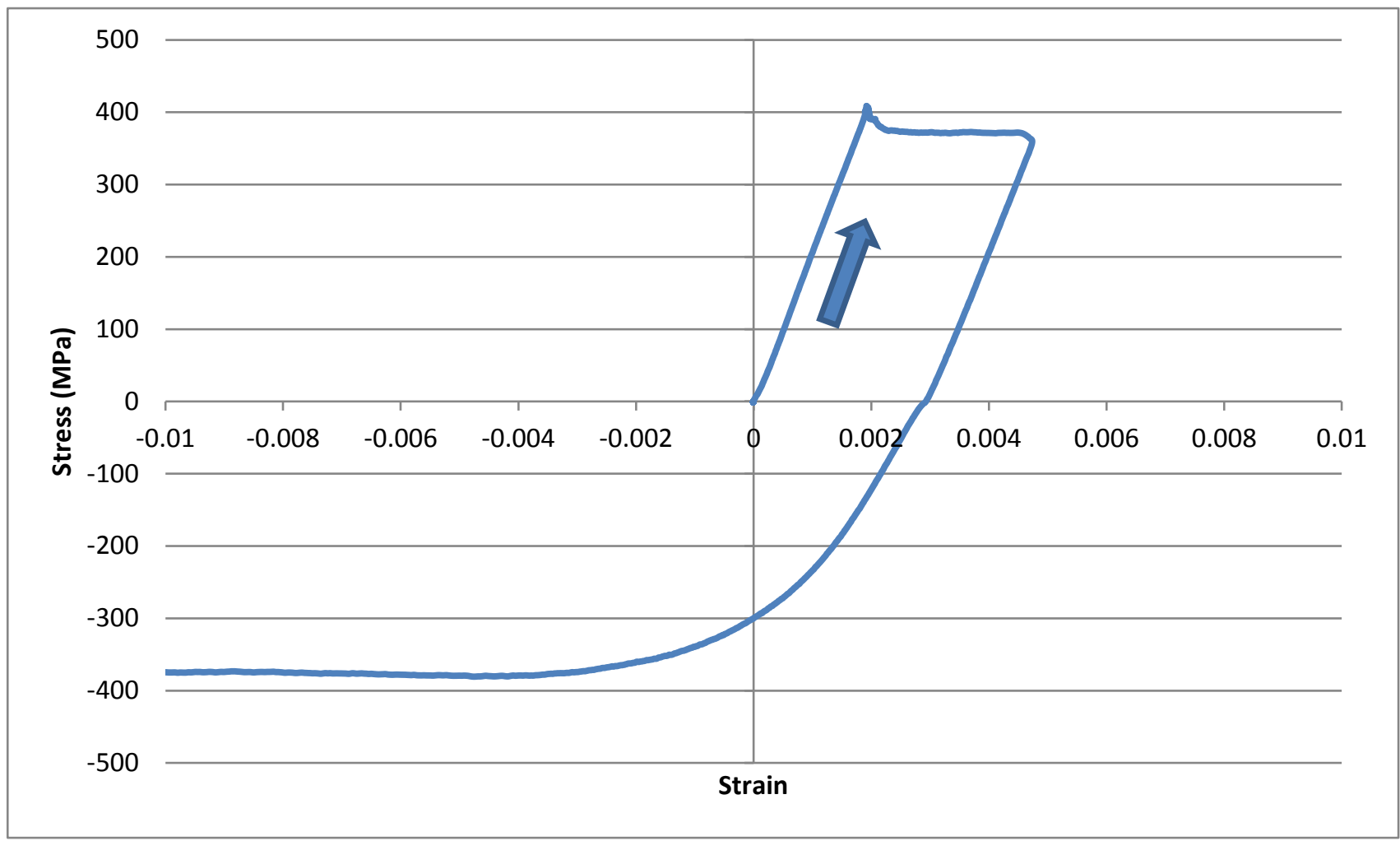

Figure 73-Graph plotting stress against strain for a tension-compression test carried out on hot-finished material-low strain

Figure 73 plots stress against strain for a low strain axial tension-compression test performed on a sample of the hot-finished material to simulate the bending process at the extrados, before compression testing. The sample was loaded in tension to 0.005 strain before the load was released; the sample was then loaded in compression. It is clear that shortly after the load is reversed (from tension to compression) that the material yields at a reduced stress and the increase in stress does not increase linearly with strain. It is clear that the material undergoes yielding at a reduced stress, however; the material quickly strain hardens. 


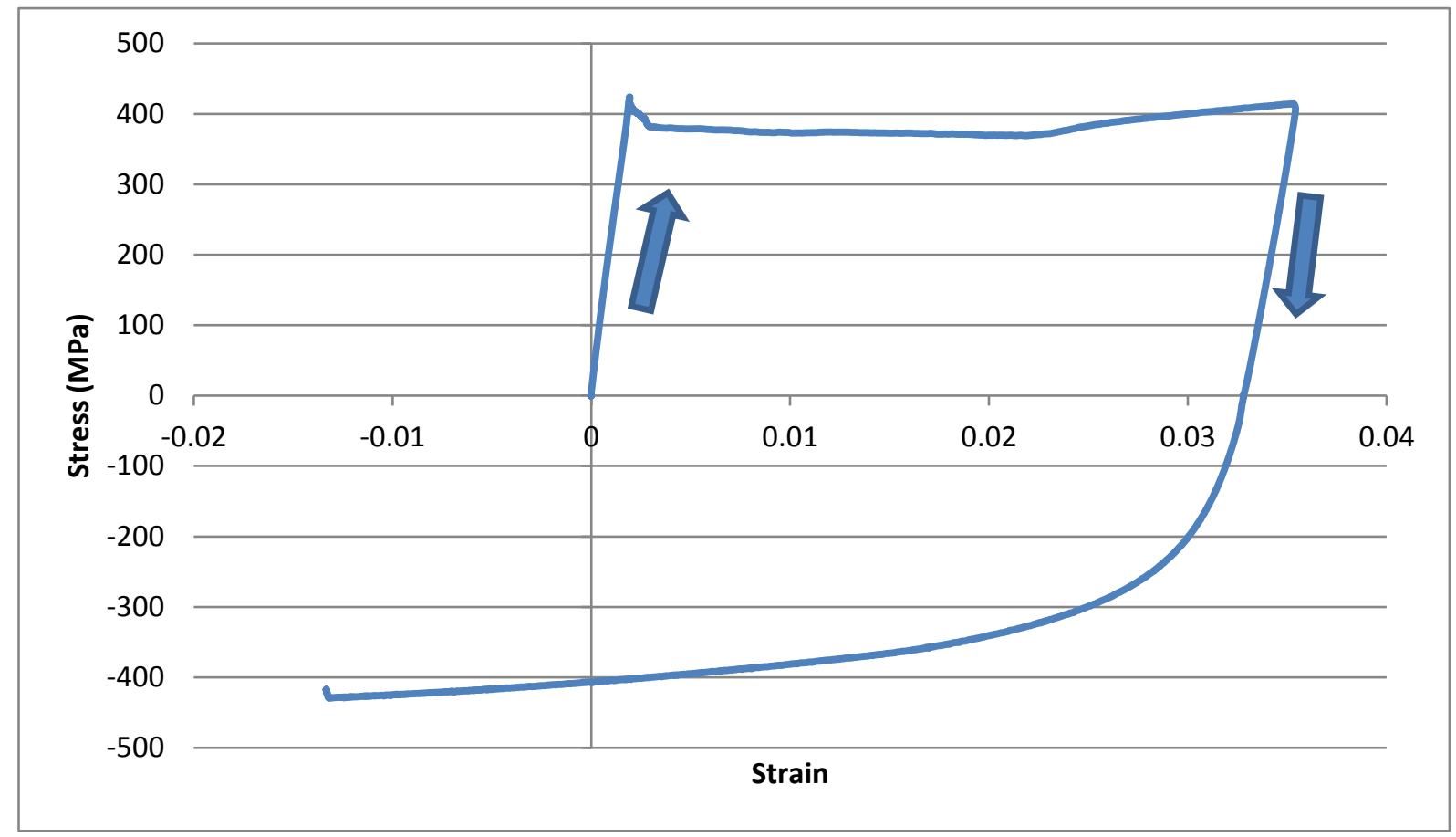

Figure 74-Graph plotting stress against strain for a tension-compression test carried out on hotfinished material-high strain

Figure 74 plots stress against strain for a high strain axial tension-compression test performed on a sample of the hot-finished material to simulate the bending process at the extrados, before compression testing. The sample was loaded in tension to 0.035 strain before the load was released; the sample was then loaded in compression. It is clear that shortly after the load is reversed (from tension to compression) that the material yields almost instantaneously and the increase in stress does not increase in line with strain linearly. It is clear that the material undergoes yielding at a reduced stress, however; the material quickly strain hardens. From this figure, it is clear that increasing the pre-strain has a greater impact on the yield strength of the material. This can be seen clearly in Figure 75 were the high and low pre-strain samples are plotted on the same axes. 


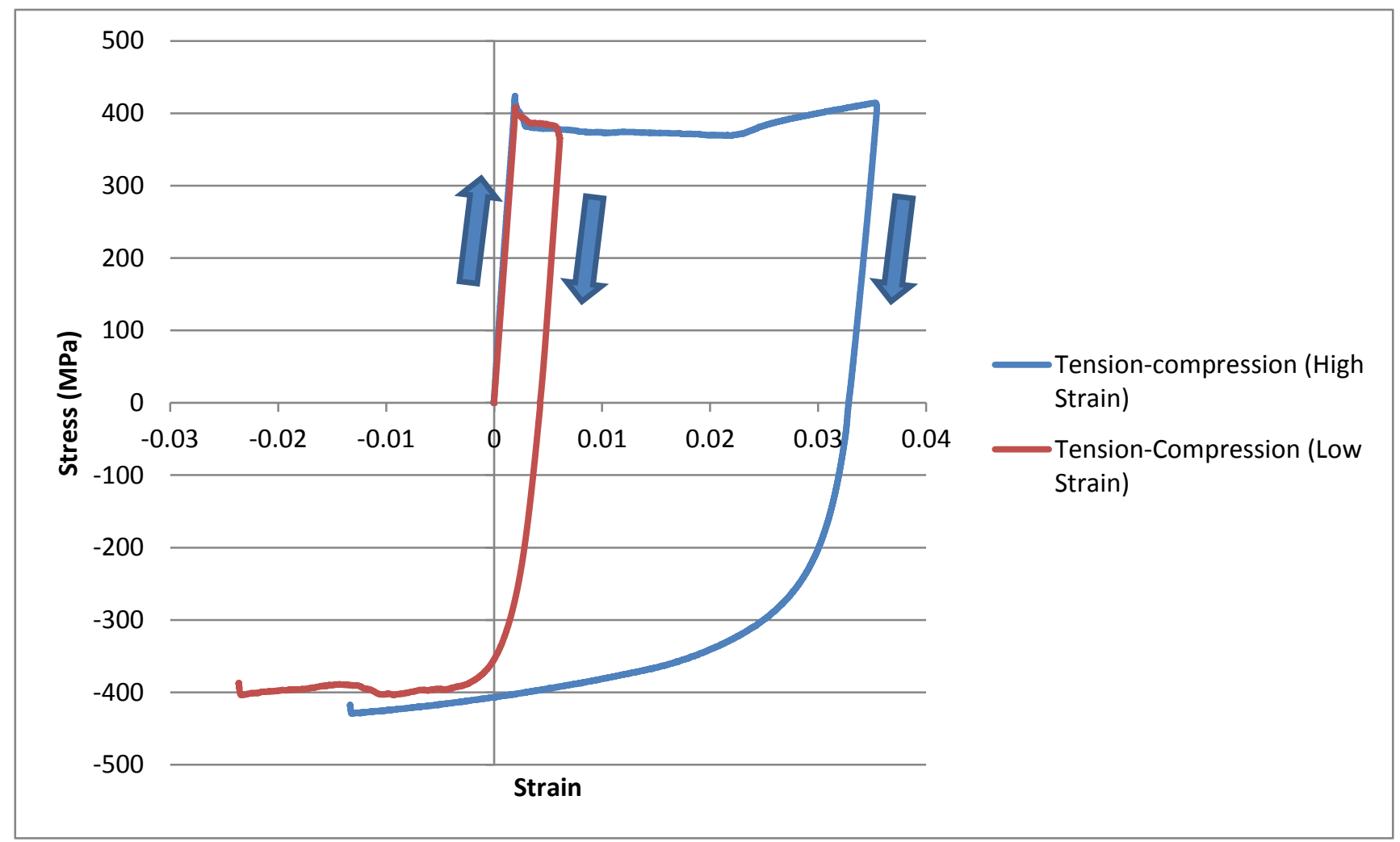

Figure 75-Graph plotting Stress and strain for a low and high strain tension-compression test

It can be seen in Figure 75 that there is a correlation between pre-strain displacement and the reduction in yield strength observed by the material. The high strain sample experiences a greater reduction in the yield stress when compared to the low strain sample. However, both samples have strain hardened to approximately the same strength by zero total strain (pre-strain has been neutralised). The virgin material starts to strain harden in tension at approximately 0.02 strain, therefore, the low strain sample has only been deformed within the discontinuous yielding zone during the tension phase and has not undergone any strain hardening before the loading was reversed.

The $0.2 \%$ proof stress of the low strain sample in compression is similar to the tension phase, only being reduced to $325 \mathrm{MPa}$, however, the $0.2 \%$ proof stress of the high strain sample is significantly less than the tension phase at approximately 200MPa. This can be seen clearly in the graph plotting modulus of stress and strain. 


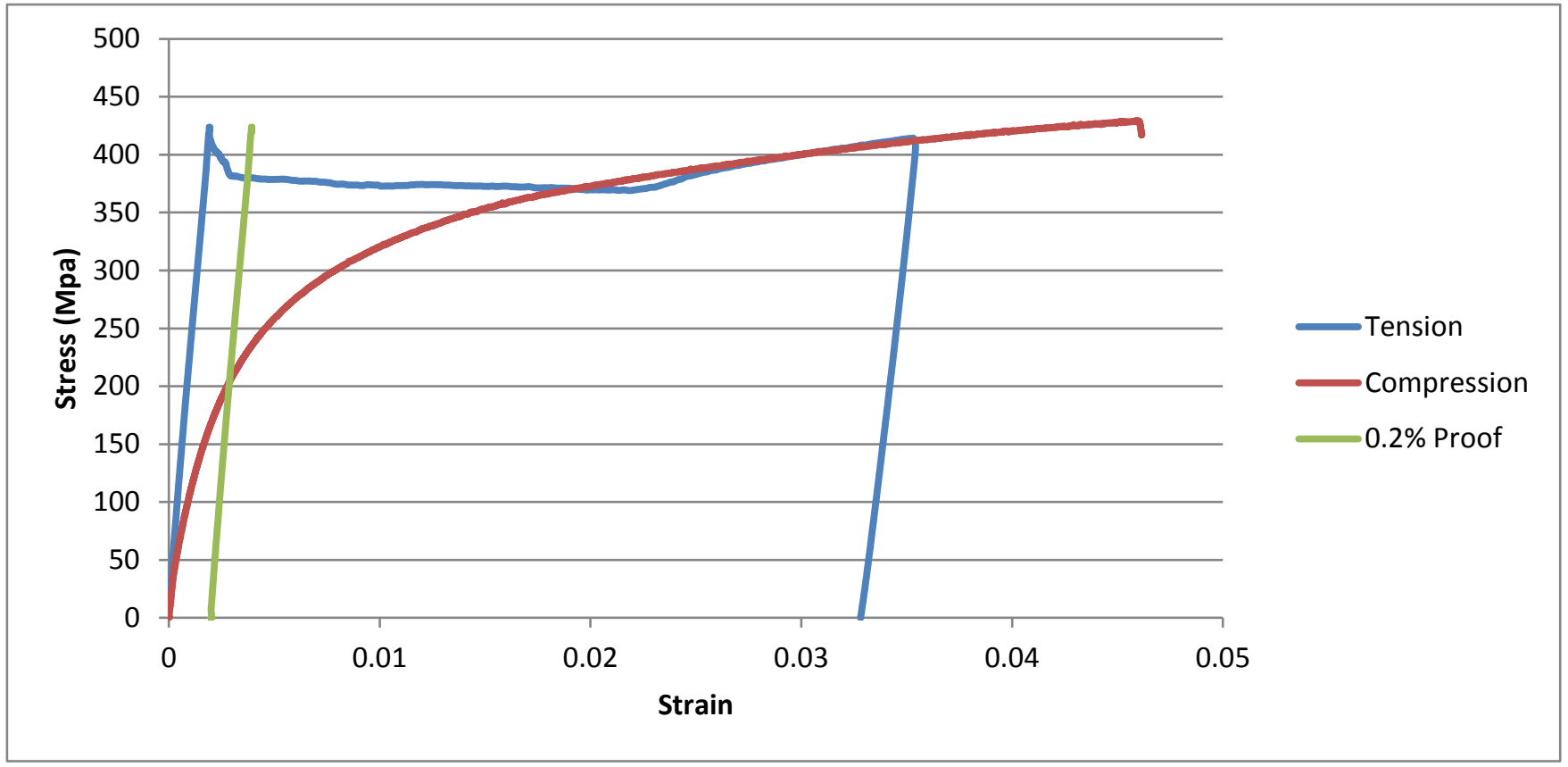

Figure 76-Graph plotting the stress and strain for a tension-compression test carried out on hot-finished material on the same axis

In Figure 76 the compression phase of the graph has been overlaid on the tension phase to depict the change in yield strength after elongation. The blue line in the tension phase of the test, the red line is the compression phase of the test. The green line is the proof stress with an offset of $0.2 \%$ strain. It can be seen that there is a significant reduction in proof stress from above the $355 \mathrm{MPa}$ requirement in tension to approximately $200 \mathrm{MPa}$ in compression. The properties of the section are restored by approximately 0.02 strain. The reason for this occurrence is dislocation movement. The dislocation density increases during tension, in the direction of the flow stress. As the load is removed and the material is loaded in compression, the dislocation density will decrease and the material will experience a reduction in yield strength. As the deformation continues, the dislocation density will start to increase again in the direction of compressive force. From the experimentation, the material has restored properties by the time the deformation in the opposite direction has matched the deformation in pre-strain.[10] 


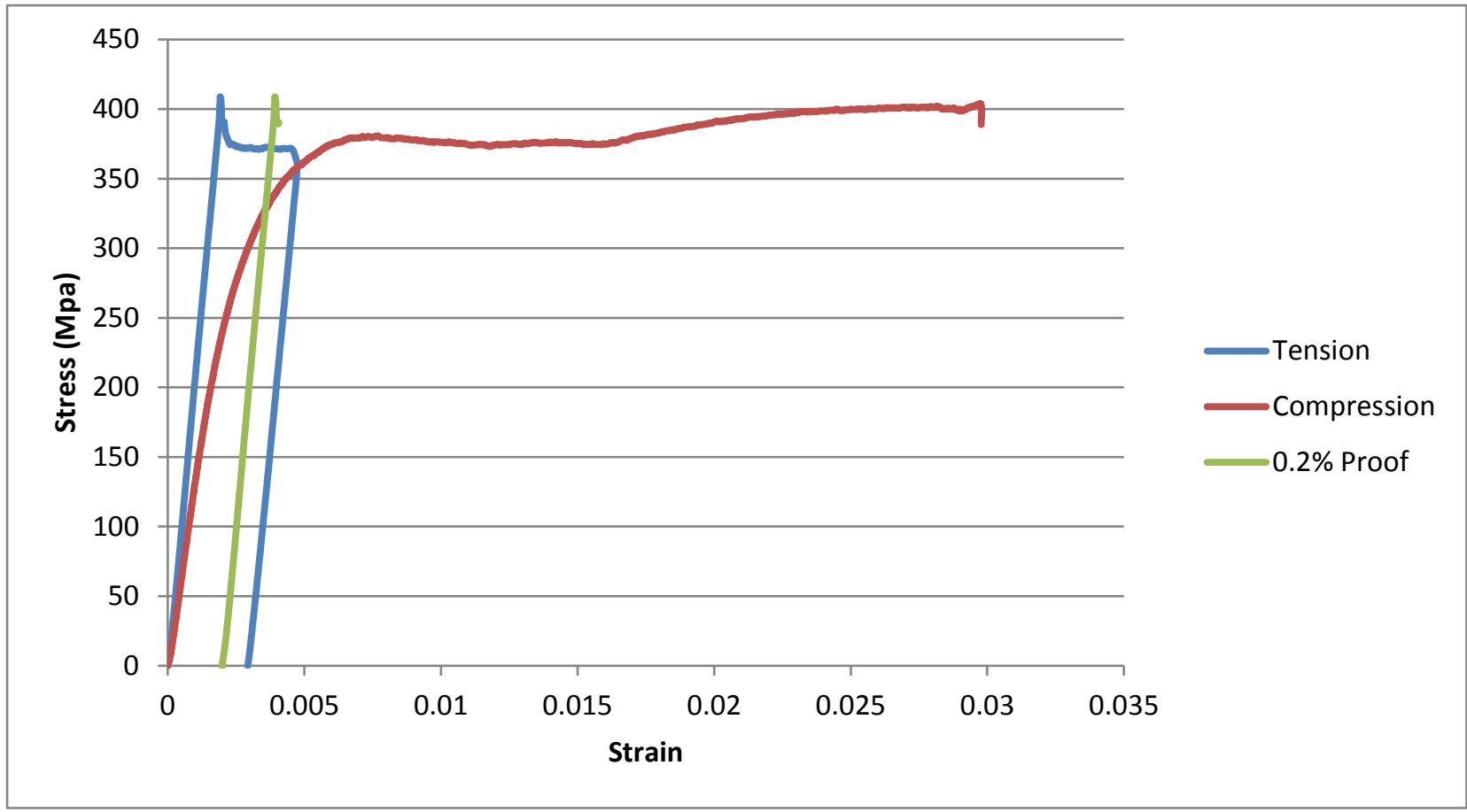

Figure 77-Graph plotting the stress and strain for a low strain tension-compression test performed on hot-finished material on the same axis

Figure 77 plots the low strain tension-compression test on a single axis. It is clear from the figure that the proof stress reduction is less than the high strain test example.

The testing performed has confirmed that areas where the loading is reversed after plastic deformation experience a reduction in yield strength. This suggests that both the intrados and the extrados would both experience a reduction in yield strength when a load is applied at 180 degrees from the original pre-stress. This equates to significantly reduced load required to yield the section when these reductions are combined.

When a straightening force is applied to the curved tube, the extrados would experience a reduction in compressive yield strength, the intrados would experience a reduction in tensile yield strength and areas not plastically deformed by the bending process (around the neutral axis) will be assisting the straitening force in order to revert to the lowest energy state. This will equate to a significantly diminished stress requirement for tube deformation. [16], [58] 


\subsubsection{Cold-formed RHS Tensile Properties}

\begin{tabular}{|c|c|c|c|c|c|c|c|c|}
\hline Sample Ref. & $\begin{array}{l}\text { Product Size } \\
(\mathrm{mm})\end{array}$ & Apparatus & $\begin{array}{l}\text { Width } \\
(\mathrm{mm})\end{array}$ & $\begin{array}{l}\text { Thickness } \\
(\mathrm{mm})\end{array}$ & $\begin{array}{l}\text { Area } \\
\left(\mathrm{mm}^{2}\right)\end{array}$ & $\begin{array}{l}\text { Yield } \\
\text { Stress } \\
\text { (MPa) }\end{array}$ & $\begin{array}{l}\text { Proof } \\
0.5 \% \\
(\mathrm{MPa})\end{array}$ & $\begin{array}{l}\text { Proof } \\
0.2 \% \\
\text { (MPa) }\end{array}$ \\
\hline $\mathrm{C} / 0.25 / \mathrm{H} \mathrm{E}$ & $150 \times 150 \times 10$ & Mayes $500 \mathrm{KN}$ & 19.97 & 9.79 & 195.5 & - & 484 & 477 \\
\hline $\mathrm{C} / 0.25 / \mathrm{H} \mathrm{I}$ & $150 \times 150 \times 10$ & Mayes 500KN & 19.95 & 9.74 & 194.3 & - & 504 & 493 \\
\hline C/0.5/H E & $150 \times 150 \times 10$ & Schenck $250 \mathrm{KN}$ & 20.09 & 9.78 & 196.5 & - & 481 & 479 \\
\hline $\mathrm{C} / 0.5 / \mathrm{H} \mathrm{I}$ & $150 \times 150 \times 10$ & Schenck 250KN & 20.09 & 9.75 & 195.9 & - & 434 & 419 \\
\hline C/0.5/H E & $150 \times 150 \times 10$ & Schenck 250KN & 20.03 & 9.92 & 198.7 & - & 475 & 459 \\
\hline $\mathrm{C} / 0.5 / \mathrm{H} \mathrm{I}$ & $150 \times 150 \times 10$ & Schenck $250 \mathrm{KN}$ & 19.93 & 9.8 & 195.3 & - & 490 & 488 \\
\hline C/1.0/H E & $150 \times 150 \times 10$ & Schenck $250 \mathrm{KN}$ & 19.97 & 9.73 & 194.3 & - & 479 & 479 \\
\hline $\mathrm{C} / 1.0 / \mathrm{H} \mathrm{I}$ & $150 \times 150 \times 10$ & Schenck $250 \mathrm{KN}$ & 19.92 & 9.77 & 194.6 & - & 406 & 390 \\
\hline C/1.0/H E & $150 \times 150 \times 10$ & Schenck 250KN & 20.09 & 9.72 & 195.3 & - & 478 & 478 \\
\hline C/1.0/H I & $150 \times 150 \times 10$ & Schenck 250KN & 20.06 & 9.82 & 197 & - & 400 & 387 \\
\hline $\mathrm{C} / 2.0 / \mathrm{H} \mathrm{E}$ & $150 \times 150 \times 10$ & Schenck 250KN & 19.91 & 9.64 & 191.9 & - & 473 & 469 \\
\hline $\mathrm{C} / 2.0 / \mathrm{H} \mathrm{I}$ & $150 \times 150 \times 10$ & Schenck 250KN & 19.92 & 9.91 & 197.4 & - & 390 & 371 \\
\hline $\mathrm{C} / 2.0 / \mathrm{H} \mathrm{E}$ & $150 \times 150 \times 10$ & Schenck $250 \mathrm{KN}$ & 20.21 & 9.59 & 193.8 & - & 477 & 473 \\
\hline C/2.0/H I & $150 \times 150 \times 10$ & Schenck 250KN & 20.3 & 9.89 & 200.8 & - & 398 & 379 \\
\hline
\end{tabular}




\begin{tabular}{|c|c|c|c|c|c|c|c|c|}
\hline C/3.0/H E & $150 \times 150 \times 10$ & Schenck $250 \mathrm{KN}$ & 19.95 & 9.61 & 191.7 & - & 476 & 471 \\
\hline $\mathrm{C} / 3.0 / \mathrm{H} \mathrm{I}$ & $150 \times 150 \times 10$ & Schenck $250 \mathrm{KN}$ & 19.9 & 9.95 & 198 & - & 386 & 370 \\
\hline C/3.0/H E & $150 \times 150 \times 10$ & Schenck 250KN & 20.08 & 9.92 & 199.2 & - & 474 & 476 \\
\hline C/3.0/H I & $150 \times 150 \times 10$ & Schenck $250 \mathrm{KN}$ & 20.06 & 9.57 & 192 & - & 378 & 362 \\
\hline C/4.0/LE & $150 \times 150 \times 10$ & Schenck 250KN & 19.97 & 9.59 & 191.5 & - & 491 & 487 \\
\hline C/4.0/L I & $150 \times 150 \times 10$ & Schenck 250KN & 19.93 & 10.05 & 200.3 & - & 406 & 391 \\
\hline C/4.0/LE & $150 \times 150 \times 10$ & Schenck 250KN & 19.92 & 9.57 & 190.6 & - & 489 & 489 \\
\hline C/4.0/L I & $150 \times 150 \times 10$ & Schenck 250KN & 19.97 & 10.05 & 200.7 & - & 395 & 385 \\
\hline C/4.0/H E & $150 \times 150 \times 10$ & Mayes 500KN & 20.09 & 9.59 & 192.7 & - & 499 & 493 \\
\hline C/4.0/H I & $150 \times 150 \times 10$ & Mayes 500KN & 20.11 & 9.97 & 200.5 & - & 463 & 445 \\
\hline C/5.0/LE & $150 \times 150 \times 10$ & Schenck 250KN & 20.05 & 9.53 & 191.1 & - & 494 & 494 \\
\hline C/5.0/L I & $150 \times 150 \times 10$ & Schenck 250KN & 20.17 & 10.15 & 204.7 & - & 384 & 380 \\
\hline
\end{tabular}

Table 8-Tensile testing values recorded for cold-formed rectangular hollow sections at the intrados and extrados 
Table 8 indicates the tensile properties of the cold-formed RHS.

It can be seen that the cold-formed material has a higher proof stress and UTS than the hot-finished product. This will be caused by the higher carbon equivalent value and cold working during production. The cold-formed material does not display the upper yield point feature; this is caused by cold work during production of the tube where it is, plastically deformed to produce the tube shape.

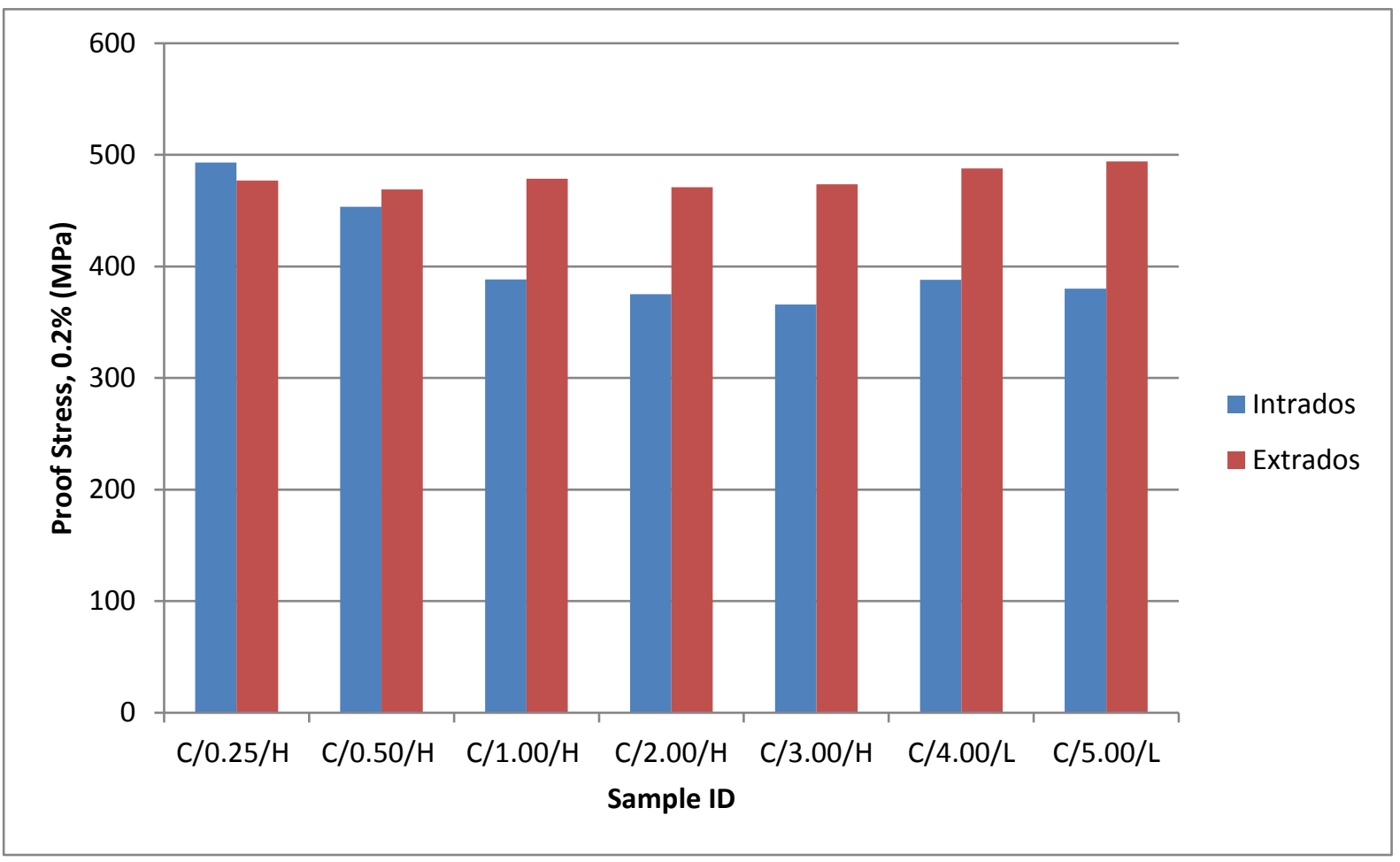

Figure 78-Graph plotting the difference in proof stress between the intrados and extrados of the cold-formed RHS with increasing strain

Figure 78 plots the intrados and extrados $0.2 \%$ proof stress for each sample from low to high strain. Like the hot-finished material, the intrados has a reduced capacity to resist tensile loading and yields at a significantly reduced stress when compared to the extrados. However, the cold product has a higher un-bend proof stress than the hot-finished equivalent and therefore these reductions do not cause the material to fail to meet specification of the unbent product after bending. The largest reduction observed during testing was $87 \mathrm{MPa}$. This reduction matches that of the hot-finished equivalent. The largest difference between the extrados and the intrados was 102MPa. This is greater than the difference observed in the hot-finished product. This increase in yield strength at the extrados is due to cold work in tension during bending. It is likely that the extrados experiences a comparable reduction in compressive yield strength due to the Bauschinger effect. This has been examined using three point bend testing 


\subsection{Three-point Bending}

Three point bend testing has been performed in order to gain a better understanding of the Bauschinger phenomenon. The aim of the test is to determine whether the Bauschinger effect occurs when the load is reversed during flexural displacement in the same manner as observed for axial displacement.

Figure 79 plots flexural stress against strain for a representative low-strain three-point bend test performed on a hot-finished sample and shows the curve for each direction that the test was carried out. Curve " $\mathrm{A}$ " was performed in the pre-stress direction. Curve " $\mathrm{B}$ " is the re-straightening phase. Curve " $\mathrm{C}$ " is the re-bending phase. The graph includes the $0.2 \%$ proof stresses for each curve. The proof stress for the unbent material, re-straitened and re-bent material is $619 \mathrm{MPa}, 503 \mathrm{MPa}$ and 534 MPa respectively. Thus, it is clear that re-straightening the test piece requires significantly less stress to yield the material and therefore less energy. This equates to a yield strength $81 \%$ of the original, unbent material. 


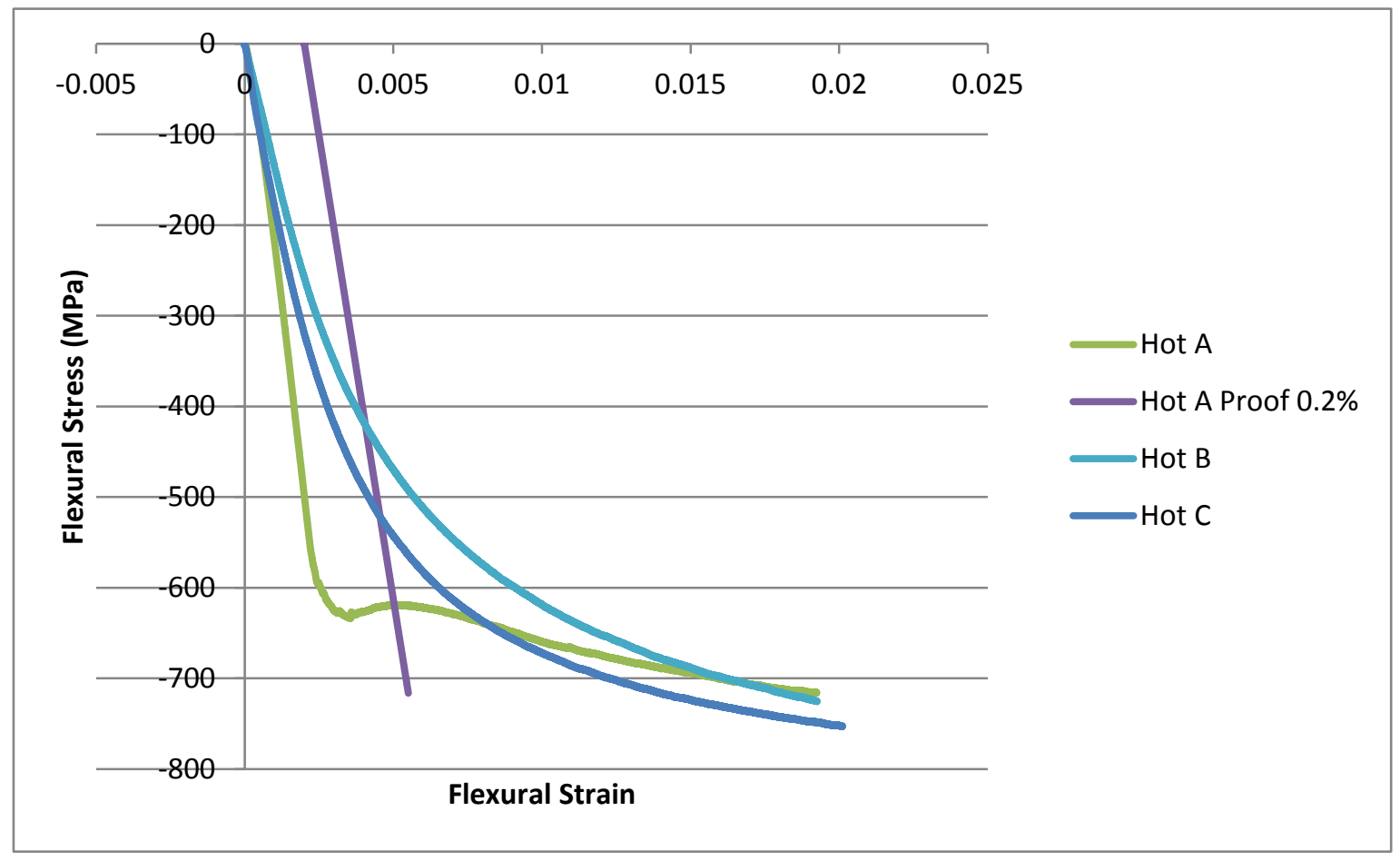

Figure 79-Graph plotting stress against strain for hot-finished, low strain, three-point bend test and $0.2 \%$ proof stresses

Figure 79 plots flexural stress against strain for a representative low-strain three-point bend test performed on a hot-finished sample and shows the curve for each direction that the test was carried out. Curve " $\mathrm{A}$ " was performed in the pre-stress direction. Curve " $\mathrm{B}$ " is the re-straightening phase. Curve " $\mathrm{C}$ " is the re-bending phase. The graph includes the $0.2 \%$ proof stresses for each curve. The proof stress for the unbent material, re-straitened and re-bent material is $619 \mathrm{MPa}, 443 \mathrm{MPa}$ and 518 MPa respectively. Thus, it is clear that re-straightening the test piece requires significantly less stress to yield the material and therefore less energy. This equates to a proof stress that is $72 \%$ of the original, unbent material. 


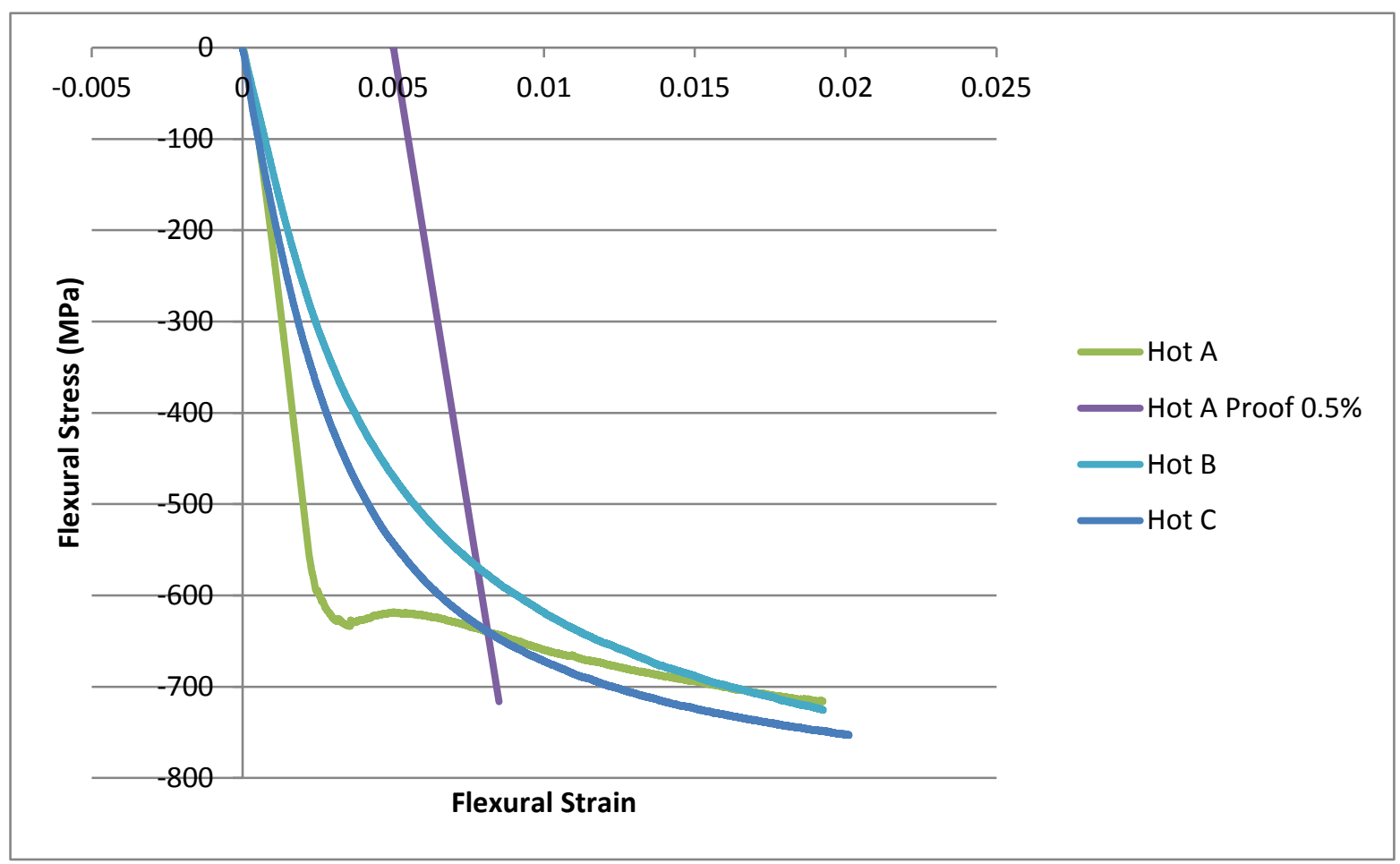

Figure 80-Graph plotting stress against strain for hot-finished, low-strain three-point bend test and $0.5 \%$ proof stresses

Figure 80 plots flexural stress against strain for a low strain three-point bend test performed on a hot-finished sample and shows the curve for each direction that the test was carried out. The graph includes the $0.5 \%$ proof stresses for each curve. The proof stress for the unbent material, restraitened and re-bent material is $637 \mathrm{MPa}, 567 \mathrm{MPa}$ and $637 \mathrm{MPa}$ respectively. Thus, it is clear that re-straightening the test piece requires significantly less stress to yield the material and therefore less energy. This equates to a proof stress that is $89 \%$ of the original, unbent material.

The difference between the proof stresses for the first bend, "Hot $A$ " is relatively small (18MPa). However, the difference between the proof stresses of the re-straightening phase are relatively large(124MPa). The reason that this occurs is that although the material yields at reduced stresses, it has an increased capacity to harden after yielding with plastic deformation and, therefore, it will recover its flexural properties as plastic deformation increases to that of the pre-strain. It has been observed that the material has recovered to that of the unbent material when the pre-strain deformation has been achieved. 


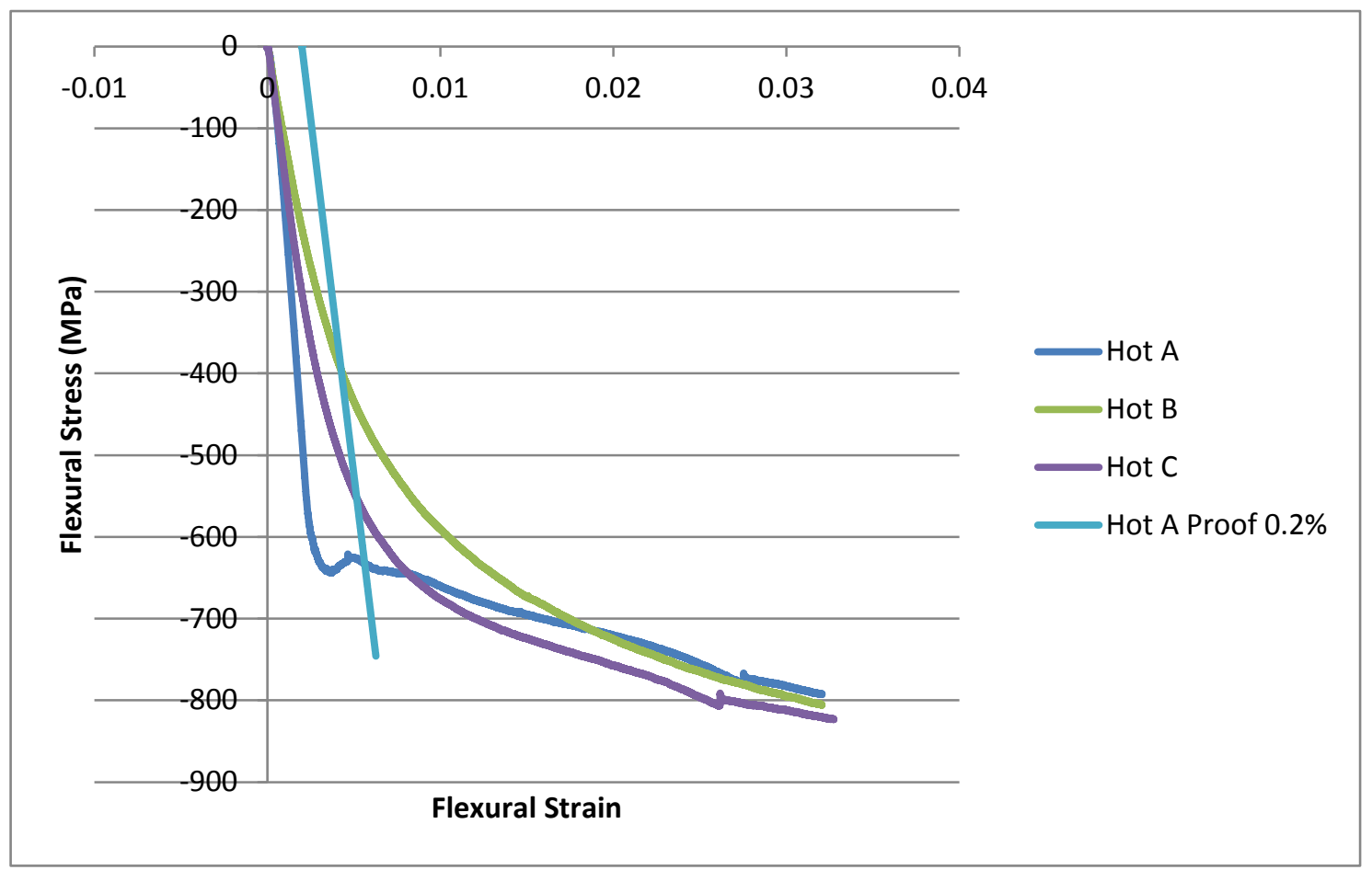

Figure 81-Graph plotting stress against strain for hot-finished, high-strain three-point bend test and $0.2 \%$ proof stresses

Figure 81 plots flexural stress against strain for a high-strain three-point bend test performed on a hot-finished sample and shows the curve for each direction that the test was carried out. The graph includes the $0.2 \%$ proof stresses for each curve. The proof stress for the unbent material, restraitened and re-bent material is $638 \mathrm{MPa}, 400 \mathrm{MPa}$ and $548 \mathrm{MPa}$ respectively. Thus, it is clear that re-straightening the test piece requires significantly less stress to yield the material and therefore less energy. This equates to a proof stress that is $63 \%$ of the original, unbent material. Increasing the pre-strain does not seem to have increased the straightening strain required for the material to strain harden to that of the bent material. The recovery takes approximately 0.02 strain.

The sample "slipped" in the apparatus during testing resulting in slight reductions in recorded stress. It is likely that this is a false reading and thus, the true stress representation would follow the original trajectory. It has been observed that the re-bending phase, "Hot C" has a lower yield and proof stress than the unbent material but rapidly increases in strength and surpasses the strength of the unbent material.

The Bauschinger stress parameters for the hot-finished material strained to 2 and $3 \%$ are:

\begin{tabular}{|l|l|l|l|l|l|l|}
\hline $\begin{array}{l}\text { Pre-strain } \\
(\%)\end{array}$ & \multicolumn{1}{|c|}{$\beta_{\sigma 1}$} & \multicolumn{1}{|c|}{$\beta_{\sigma 2}$} & $\beta_{\sigma 3}$ & $\beta_{\sigma 4}$ & $\beta_{\varepsilon}$ & $\beta_{E}$ \\
\hline 2 & 0.97 & 0.39 & 0.22 & 0.78 & 0.83 & 0.15 \\
\hline 3 & 0.96 & 0.49 & 0.34 & 0.66 & 0.47 & 0.14 \\
\hline
\end{tabular}


According to Muir and Abel, the stress parameter, 1 which can be seen in Figure 19, increases rapidly to a pre-strain of 0.001 after which the rate is diminished. The strain parameter initially decreases rapidly with increasing pre-strain, however, as with the stress parameter; the rate of change is reduced at approximately 0.001 strain. The energy parameter initially decreases with increasing prestrain, however, the rate of decreases significantly at approximately 0.001 pre-strain. The parameters recorded from testing the TATA Steel material differ to those recorded my Muir and Abel. All of the parameters decrease with increasing pre-strain. 


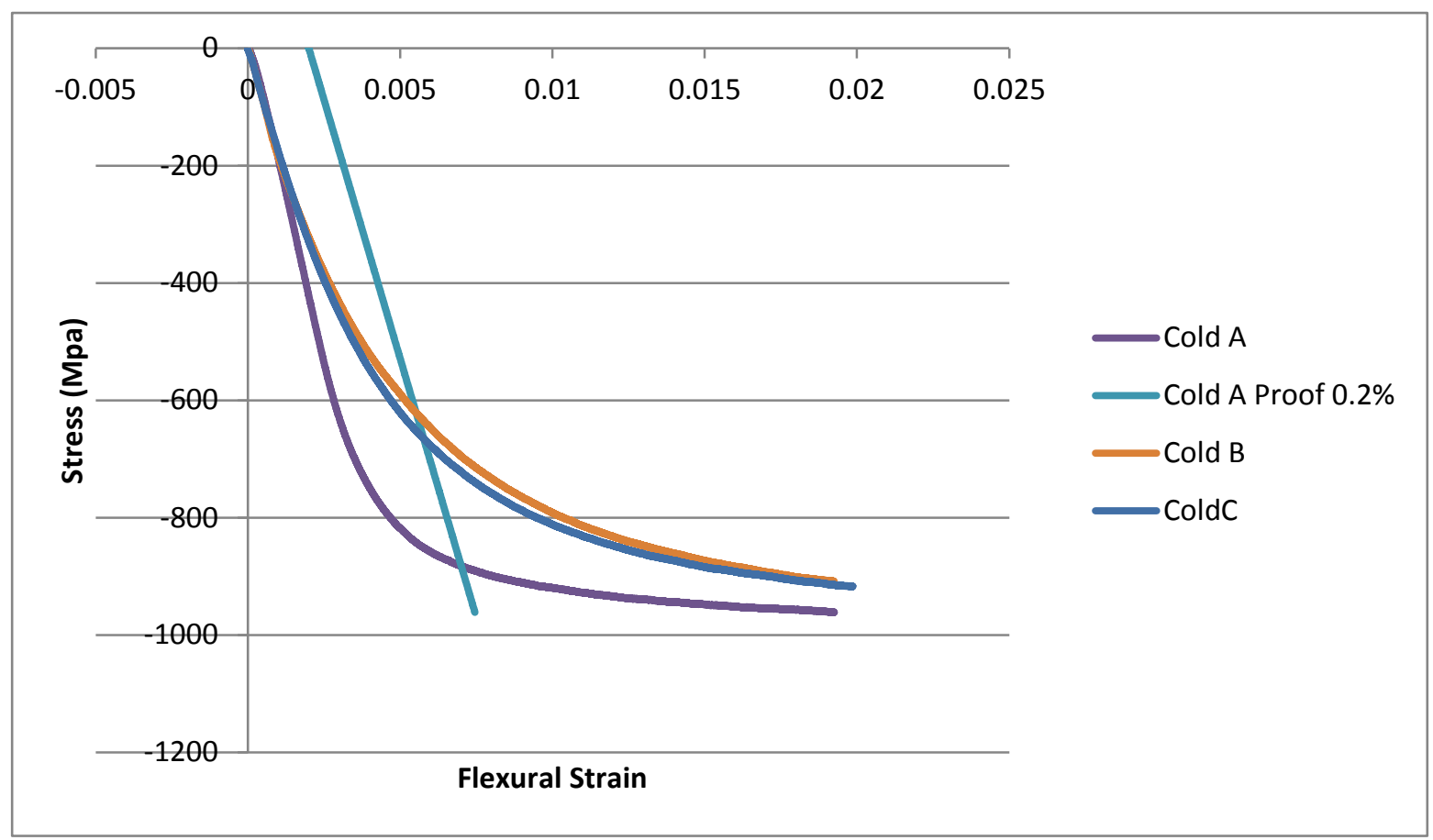

Figure 82-Graph plotting stress against strain for cold-formed, low strain three-point bend test and $0.2 \%$ proof stresses

Figure 82 plots flexural stress against strain for a low-strain three-point bend test performed on a cold-formed sample and shows the curve for each direction that the test was carried out. The graph includes the $0.2 \%$ proof stresses for each curve. As expected there is no upper yield point present on the curve. This is due to the material having already undergone plastic deformation during the production of the material. The proof stress for the unbent material, re-straitened and re-bent material is $879 \mathrm{MPa}, 634 \mathrm{MPa}$ and $680 \mathrm{MPa}$ respectively. Thus, it is clear that re-straightening the test piece requires significantly less stress to yield the material and therefore less energy. This equates to a proof stress that is $72 \%$ of the original, unbent material. It has been observed that the rebending phase, "Hot C" has a lower yield and proof stress than the unbent material but greater than that of the re-straitened material. 


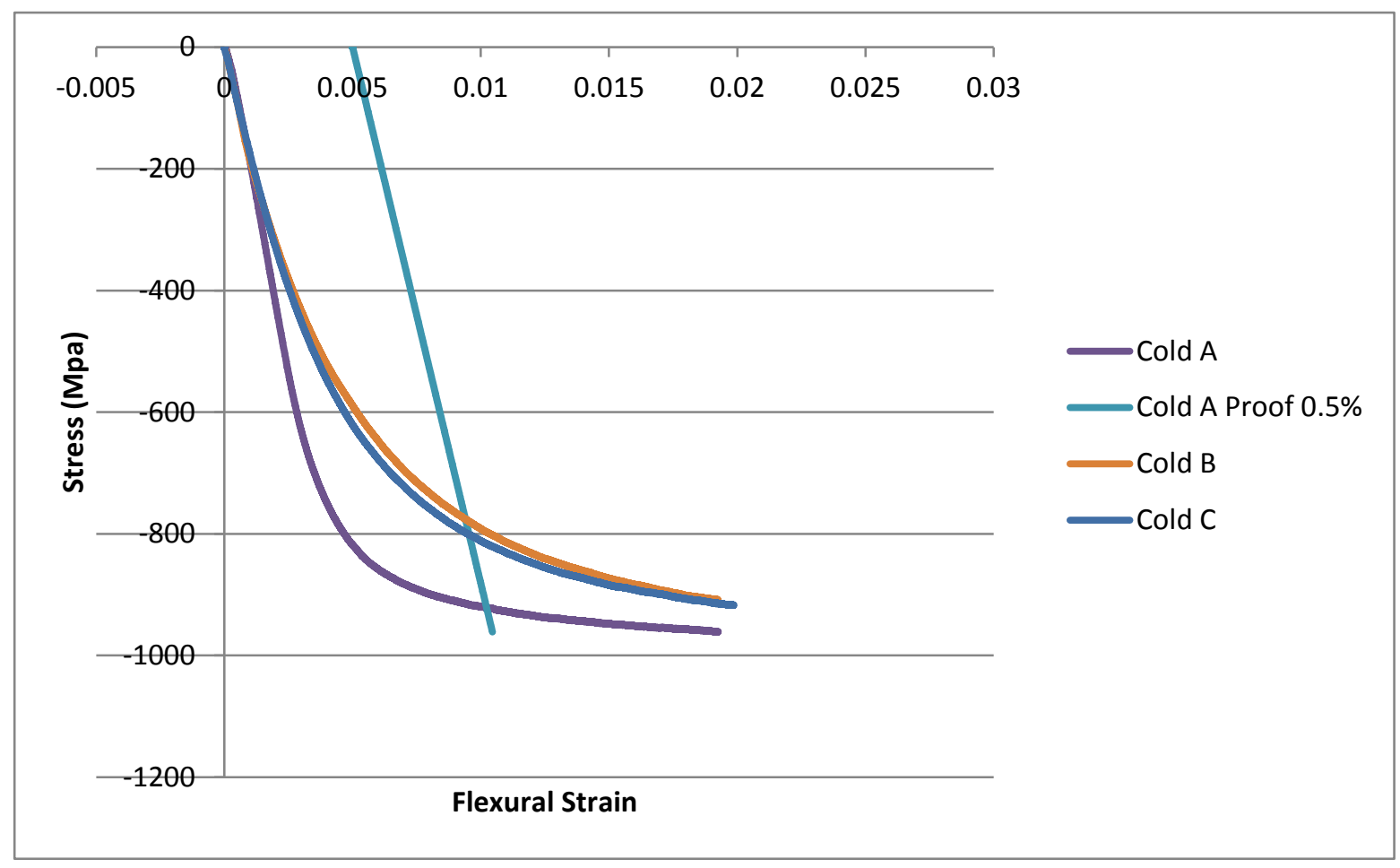

Figure 83-Graph plotting stress against strain for cold-formed, low strain three-point bend test and $\mathbf{0 . 5 \%}$ proof stresses carried out

Figure 83 plots flexural stress against strain for a low strain 3 point bend test performed on a coldformed sample and shows the curve for each direction that the test was carried out. The graph includes the $0.5 \%$ proof stresses for each curve. The proof stress for the unbent material, restraitened and re-bent material is $915 \mathrm{MPa}, 795 \mathrm{MPa}$ and $800 \mathrm{MPa}$ respectively. Thus, it is clear that re-straightening the test piece requires significantly less stress to yield the material and therefore less energy. This equates to a proof stress that is $87 \%$ of the original, unbent material.

The difference between the proof stresses for the first bend, "Cold A" is relatively small (36MPa). However, the difference between the proof stresses of the re-straightening phase are relatively large (161MPa). The reason for this, as with the hot-finished material is that although the material yields at reduced stresses, it has an increased capacity to harden after yielding with plastic deformation and, therefore, it will recover its flexural properties as plastic deformation increases to that of the pre-strain. It has been observed that the material has recovered to that of the unbent material when the pre-strain deformation has been achieved.

It has been observed that unlike the hot-finished material, the cold-formed material does not recovered to that of the unbent material when the pre-strain deformation has been achieved. 


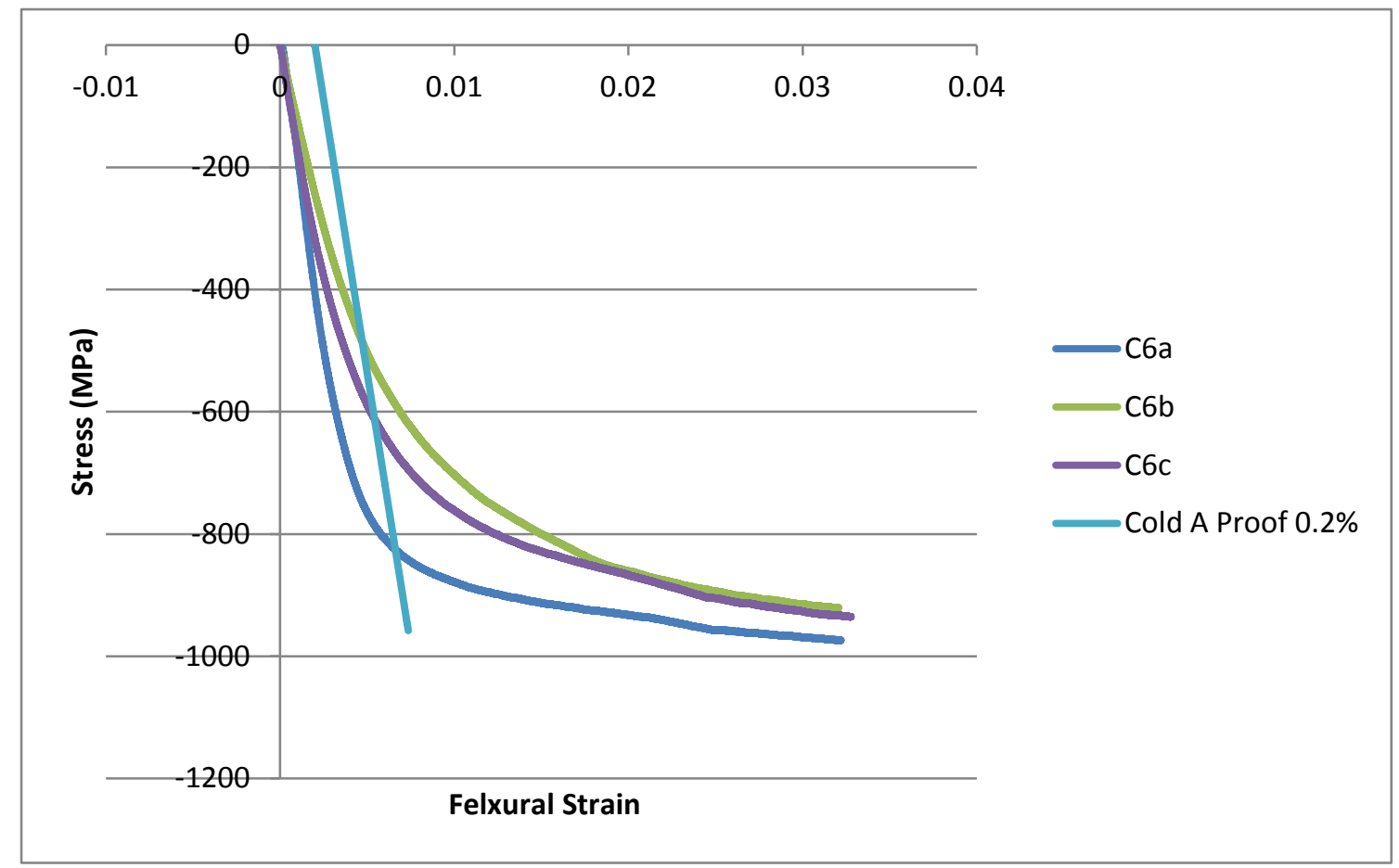

Figure 84-Graph plotting stress against strain for cold-formed, high-strain three-point bend test and $0.2 \%$ proof stresses carried out

Figure 84 plots flexural stress against strain for a high-strain three-point bend test performed on a cold-formed sample and shows the curve for each direction that the test was carried out. The graph includes the $0.2 \%$ proof stresses for each curve. The proof stress for the unbent material, restraitened and re-bent material is $830 \mathrm{MPa}, 510 \mathrm{MPa}$ and $613 \mathrm{MPa}$ respectively. Thus, it is clear that re-straightening the test piece requires significantly less stress to yield the material and therefore less energy. This equates to a yield strength $61 \%$ of the original, unbent material. Due to the larger pre-strain performed on this sample, more deformation is required in order to achieve a strength similar to that of the unbent material. However, unlike the hot-finished samples, neither the high or low strain cold formed samples tested fully recover to the strength of the initial bend.

It has been observed that the re-bending phase, "cold C" has a lower yield and proof stress than the unbent material but greater than the re-straightened material.

The three-point bend testing has confirmed that the Bauschinger effect occurs for both axial and flexural deformation. Therefore, it can be concluded that the reduced yield and proof stress observed during tensile testing at the intrados was caused by the Bauschinger effect. It is also logical to assume that the extrados would experience a similar reduction in proof stress when tested in compression. 


\begin{tabular}{|l|l|l|l|l|l|l|}
\hline $\begin{array}{l}\text { Pre-strain } \\
(\%)\end{array}$ & \multicolumn{1}{|c|}{$\beta_{\sigma 1}$} & \multicolumn{1}{|c|}{$\beta_{\sigma 2}$} & $\beta_{\sigma 3}$ & $\beta_{\sigma 4}$ & $\beta_{\varepsilon}$ & $\beta_{E}$ \\
\hline 2 & 0.84 & 0.41 & 0.23 & 0.77 & N/A & N/A \\
\hline 3 & 0.95 & 0.48 & 0.30 & 0.70 & N/A & N/A \\
\hline
\end{tabular}

The stress parameter for the cold-formed material increases in line with Muir and Abel's work, however, it was not possible to determine the strain and energy parameters as the stress did not reach that of the pre-strain within the same strain period. 


\subsection{Impact Testing}

\subsubsection{Hot-finished RHS Charpy Impact Resistance Properties}

\begin{tabular}{|c|c|c|c|c|c|c|}
\hline Sample Reference. & $\begin{array}{l}\text { Product Size } \\
(\mathrm{mm})\end{array}$ & Test Machine & $\begin{array}{l}\text { Sample } \\
\text { Size (mm) }\end{array}$ & $\begin{array}{l}\text { Test } \\
\text { Temp }\left({ }^{\circ} \mathrm{C}\right)\end{array}$ & $\begin{array}{l}\text { Individual } \\
\text { Values (J) }\end{array}$ & $\begin{array}{l}\text { Average } \\
\text { Value (J) }\end{array}$ \\
\hline H/0.0/H E & $150 \times 150 \times 10$ & SANS 450J & $10 \times 7.5$ & -20 & $173-175-175$ & 174 \\
\hline $\mathrm{H} / 0.0 / \mathrm{H} \mathrm{I}$ & $150 \times 150 \times 10$ & SANS 450J & $10 \times 7.5$ & -20 & $168-167-183$ & 173 \\
\hline H/0.25/HE & $150 \times 150 \times 10$ & SANS 450J & $10 \times 7.5$ & -20 & $181-172-166$ & 173 \\
\hline $\mathrm{H} / 0.25 / \mathrm{H} \mathrm{I}$ & $150 \times 150 \times 10$ & SANS 450J & $10 \times 7.5$ & -20 & 167-184-197 & 183 \\
\hline H/0.5/H E & $150 \times 150 \times 10$ & SANS 450J & $10 \times 7.5$ & -20 & $168-177-164$ & 170 \\
\hline $\mathrm{H} / 0.5 / \mathrm{H} \mathrm{I}$ & $150 \times 150 \times 10$ & SANS 450J & $10 \times 7.5$ & -20 & 176-177-168 & 174 \\
\hline H/1.0/H E & $150 \times 150 \times 10$ & SANS 450J & $10 \times 7.5$ & -20 & $188-164-177$ & 176 \\
\hline H/1.0/H I & $150 \times 150 \times 10$ & SANS 450J & $10 \times 7.5$ & -20 & $169-181-175$ & 171 \\
\hline H/1.0/HE & $150 \times 150 \times 10$ & SANS 450J & $10 \times 7.5$ & -20 & $162-175-194$ & 177 \\
\hline H/1.0/H I & $150 \times 150 \times 10$ & SANS 450J & $10 \times 7.5$ & -20 & $179-167-173$ & 173 \\
\hline H/2.0/HE & $150 \times 150 \times 10$ & SANS 450J & $10 \times 7.5$ & -20 & 173-161-178 & 171 \\
\hline $\mathrm{H} / 2.0 / \mathrm{H} \mathrm{I}$ & $150 \times 150 \times 10$ & SANS 450J & $10 \times 7.5$ & -20 & $169-176-174$ & 173 \\
\hline H/3.0/H E & $150 \times 150 \times 10$ & SANS 450J & $10 \times 7.5$ & -20 & 179-183-198 & 187 \\
\hline H/3.0/H I & $150 \times 150 \times 10$ & SANS 450J & $10 \times 7.5$ & -20 & $167-172-164$ & 168 \\
\hline H/4.0/H E & $150 \times 150 \times 10$ & SANS 450J & $10 \times 7.5$ & -20 & $160-165-163$ & 163 \\
\hline H/4.0/H I & $150 \times 150 \times 10$ & SANS 450J & $10 \times 7.5$ & -20 & 161-173-102 & 145 \\
\hline H/4.0/L E & $150 \times 150 \times 10$ & SANS 450J & $10 \times 7.5$ & -20 & 179-184-159 & 174 \\
\hline H/4.0/L I & $150 \times 150 \times 10$ & SANS 450J & $10 \times 7.5$ & -20 & $172-168-180$ & 173 \\
\hline H/4.0/L E & $150 \times 150 \times 10$ & SANS 450J & $10 \times 7.5$ & -20 & 165-163-168 & 165 \\
\hline 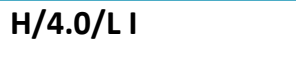 & $150 \times 150 \times 10$ & SANS 450J & $10 \times 7.5$ & -20 & $157-159-159$ & 158 \\
\hline H/5.0/L E & $150 \times 150 \times 10$ & SANS 450J & $10 \times 7.5$ & -20 & $190-164-165$ & 173 \\
\hline 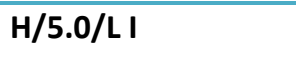 & $150 \times 150 \times 10$ & SANS 450J & $10 \times 7.5$ & -20 & $165-156-167$ & 163 \\
\hline H/5.0/L E & $150 \times 150 \times 10$ & SANS 450J & $10 \times 7.5$ & -20 & $159-165-164$ & 163 \\
\hline H/5.0/L I & $150 \times 150 \times 10$ & SANS 450J & $10 \times 7.5$ & -20 & $161-160-157$ & 159 \\
\hline
\end{tabular}

Table 9-Charpy impact resistance values for cold-formed rectangular hollow sections at the intrados and extrados

Only samples marked with a * were completely broken in the test 
It can be seen from Table 9 that the hot-finished material has a good impact resistance for the sample dimensions $(10 \times 7.5 \mathrm{~mm})$. The range of values recorded is $96 \mathrm{~J}$, however; the majority of the values recorded are above 155J. There is little change in impact resistance as strain rate is increased. It is not known whether there is any effect on impact resistance caused by the bending process within the range of strains used in this project. There is no clear variation between the intrados and extrados and their relationship with impact resistance. 


\subsubsection{Cold-formed RHS Charpy Impact Resistance Properties}

\begin{tabular}{|c|c|c|c|c|c|c|}
\hline Sample Reference. & $\begin{array}{l}\text { Product Size } \\
(\mathrm{mm})\end{array}$ & Test Machine & $\begin{array}{l}\text { Sample } \\
\text { Size (mm) }\end{array}$ & $\begin{array}{l}\text { Test } \\
\text { Temp }\left({ }^{\circ} \mathrm{C}\right)\end{array}$ & $\begin{array}{l}\text { Individual Values } \\
\text { (J) }\end{array}$ & $\begin{array}{l}\text { Average Value } \\
\text { (J) }\end{array}$ \\
\hline C/0.0/H E & $150 \times 150 \times 10$ & SANS 450J & $10 \times 7.5$ & -20 & $100-97-118$ & 105 \\
\hline $\mathrm{C} / 0.0 / \mathrm{H} \mathrm{I}$ & $150 \times 150 \times 10$ & SANS 450J & $10 \times 7.5$ & -20 & 97-99-104 & 100 \\
\hline $\mathrm{C} / 0.25 / \mathrm{H} \mathrm{E}$ & $150 \times 150 \times 10$ & SANS 450J & $10 \times 7.5$ & -20 & $80-56-81$ & 72 \\
\hline $\mathrm{C} / 0.25 / \mathrm{H} \mathrm{I}$ & $150 \times 150 \times 10$ & SANS 450J & $10 \times 7.5$ & -20 & $116-113-110$ & 113 \\
\hline C/0.5/H E & $150 \times 150 \times 10$ & SANS 450J & $10 \times 7.5$ & -20 & $86-90-12$ & 63 \\
\hline $\mathrm{C} / 0.5 / \mathrm{H} \mathrm{I}$ & $150 \times 150 \times 10$ & SANS 450J & $10 \times 7.5$ & -20 & $78-76-72$ & 75 \\
\hline $\mathrm{C} / 0.5 / \mathrm{H} \mathrm{E}$ & $150 \times 150 \times 10$ & SANS 450J & $10 \times 7.5$ & -20 & $19 *-25^{*}-57$ & 34 \\
\hline $\mathrm{C} / 0.5 / \mathrm{H} \mathrm{I}$ & $150 \times 150 \times 10$ & SANS 450J & $10 \times 7.5$ & -20 & $70-122-112$ & 101 \\
\hline $\mathrm{C} / 1.0 / \mathrm{HE}$ & $150 \times 150 \times 10$ & SANS 450J & $10 \times 7.5$ & -20 & $84-87-35 *$ & 69 \\
\hline C/1.0/H I & $150 \times 150 \times 10$ & SANS 450J & $10 \times 7.5$ & -20 & 103-127-113 & 114 \\
\hline C/1.0/H E & $150 \times 150 \times 10$ & SANS 450J & $10 \times 7.5$ & -20 & 73-120-92 & 95 \\
\hline C/1.0/H I & $150 \times 150 \times 10$ & SANS 450J & $10 \times 7.5$ & -20 & $115-118-116$ & 116 \\
\hline $\mathrm{C} / 2.0 / \mathrm{H} \mathrm{E}$ & $150 \times 150 \times 10$ & SANS 450J & $10 \times 7.5$ & -20 & $101-62-68$ & 77 \\
\hline C/2.0/H I & $150 \times 150 \times 10$ & SANS 450J & $10 \times 7.5$ & -20 & $103-70-98$ & 90 \\
\hline $\mathrm{C} / 2.0 / \mathrm{H} \mathrm{E}$ & $150 \times 150 \times 10$ & SANS 450J & $10 \times 7.5$ & -20 & $166-69 *-91$ & 109 \\
\hline C/2.0/H I & $150 \times 150 \times 10$ & SANS 450J & $10 \times 7.5$ & -20 & $103-120-92$ & 111 \\
\hline C/3.0/H E & $150 \times 150 \times 10$ & SANS 450J & $10 \times 7.5$ & -20 & $96-107-106$ & 103 \\
\hline C/3.0/H I & $150 \times 150 \times 10$ & SANS 450J & $10 \times 7.5$ & -20 & $99-32-88$ & 73 \\
\hline $\mathrm{C} / 3.0 / \mathrm{HE}$ & $150 \times 150 \times 10$ & SANS 450J & $10 \times 7.5$ & -20 & $57-58-65$ & 60 \\
\hline C/3.0/H I & $150 \times 150 \times 10$ & SANS 450J & $10 \times 7.5$ & -20 & 61-101-64 & 75 \\
\hline C/4.0/H E & $150 \times 150 \times 10$ & SANS 450J & $10 \times 7.5$ & -20 & $73-78-92$ & 81 \\
\hline C/4.0/H I & $150 \times 150 \times 10$ & SANS 450J & $10 \times 7.5$ & -20 & 30-92-59- & 60 \\
\hline C/4.0/L E & $150 \times 150 \times 10$ & SANS 450J & $10 \times 7.5$ & -20 & $65-86-62$ & 71 \\
\hline C/4.0/L I & $150 \times 150 \times 10$ & SANS 450J & $10 \times 7.5$ & -20 & 123-104-106 & 111 \\
\hline C/4.0/L E & $150 \times 150 \times 10$ & SANS 450J & $10 \times 7.5$ & -20 & $76-35-18$ & 43 \\
\hline C/4.0/L I & $150 \times 150 \times 10$ & SANS 450J & $10 \times 7.5$ & -20 & $113-114-100$ & 109 \\
\hline $\mathrm{C} / 5.0 / \mathrm{L} \mathrm{E}$ & $150 \times 150 \times 10$ & SANS 450J & $10 \times 7.5$ & -20 & $85-63-77$ & 75 \\
\hline C/5.0/L I & $150 \times 150 \times 10$ & SANS 450J & $10 \times 7.5$ & -20 & 71-98-101 & 90 \\
\hline
\end{tabular}

Table 10-Charpy impact resistance values for cold-formed rectangular hollow sections at the intrados and extrados 
The cold-formed impact resistance can be seen in Table 10. The results are less consistent than the hot-finished equivalent and vary significantly. The majority of the results are significantly lower than those of the hot-finished material. The range of values is slightly larger (110J), however; the distribution is more varied than the hot-finished material. There is no clear evidence from the testing performed that bending strain has any effect on the impact resistance of the cold-formed product.

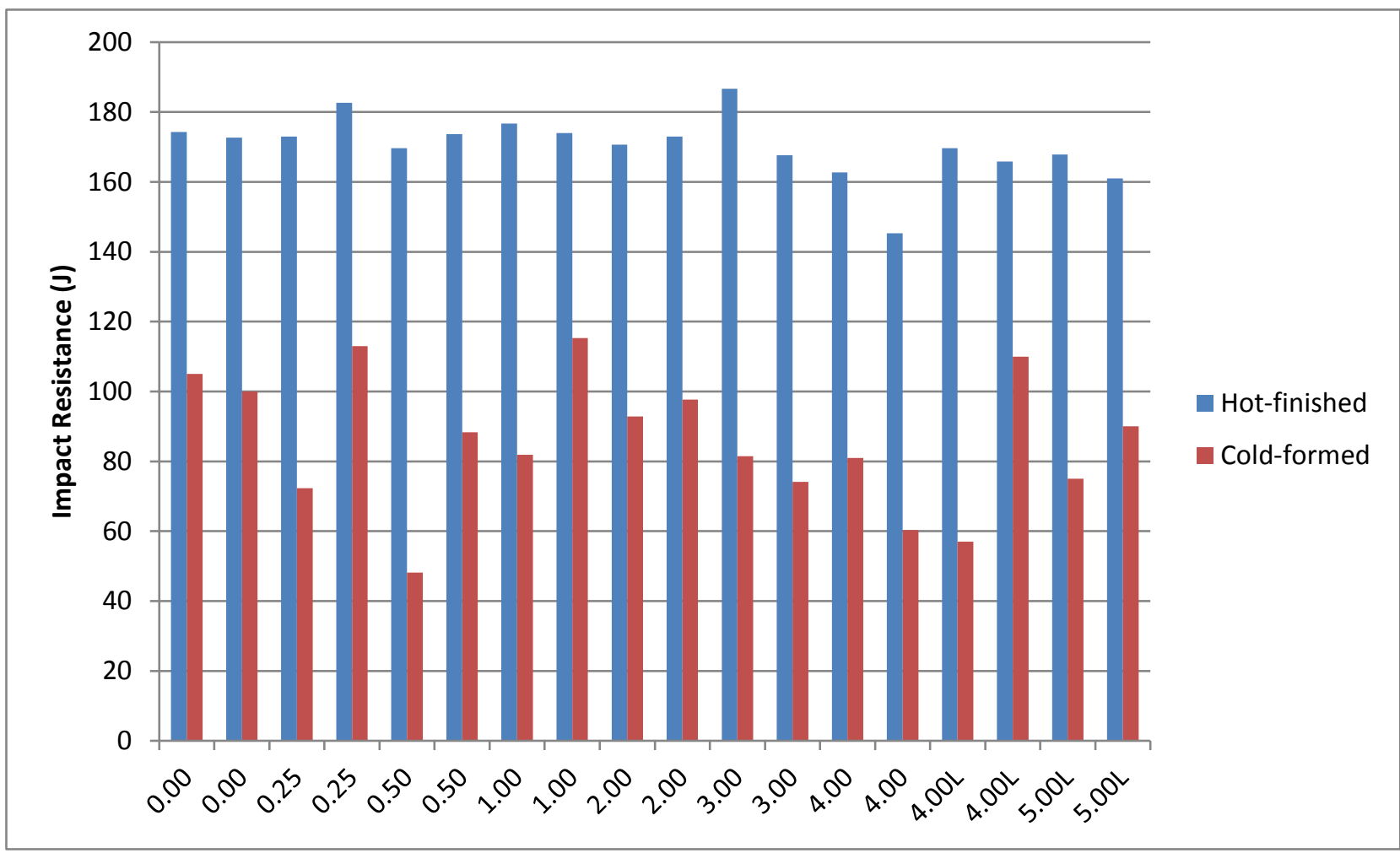

\section{Figure 85-Graph plotting mean Charpy impact resistance for the hot-finished material}

It can be seen in Figure 85 that the impact resistance of the hot-finished material is superior to the cold-formed equivalent. The unbent hot-finished material is approximately $70 \mathrm{~J}$ tougher than the cold-formed tube. 


\subsection{Physical Dimensions}

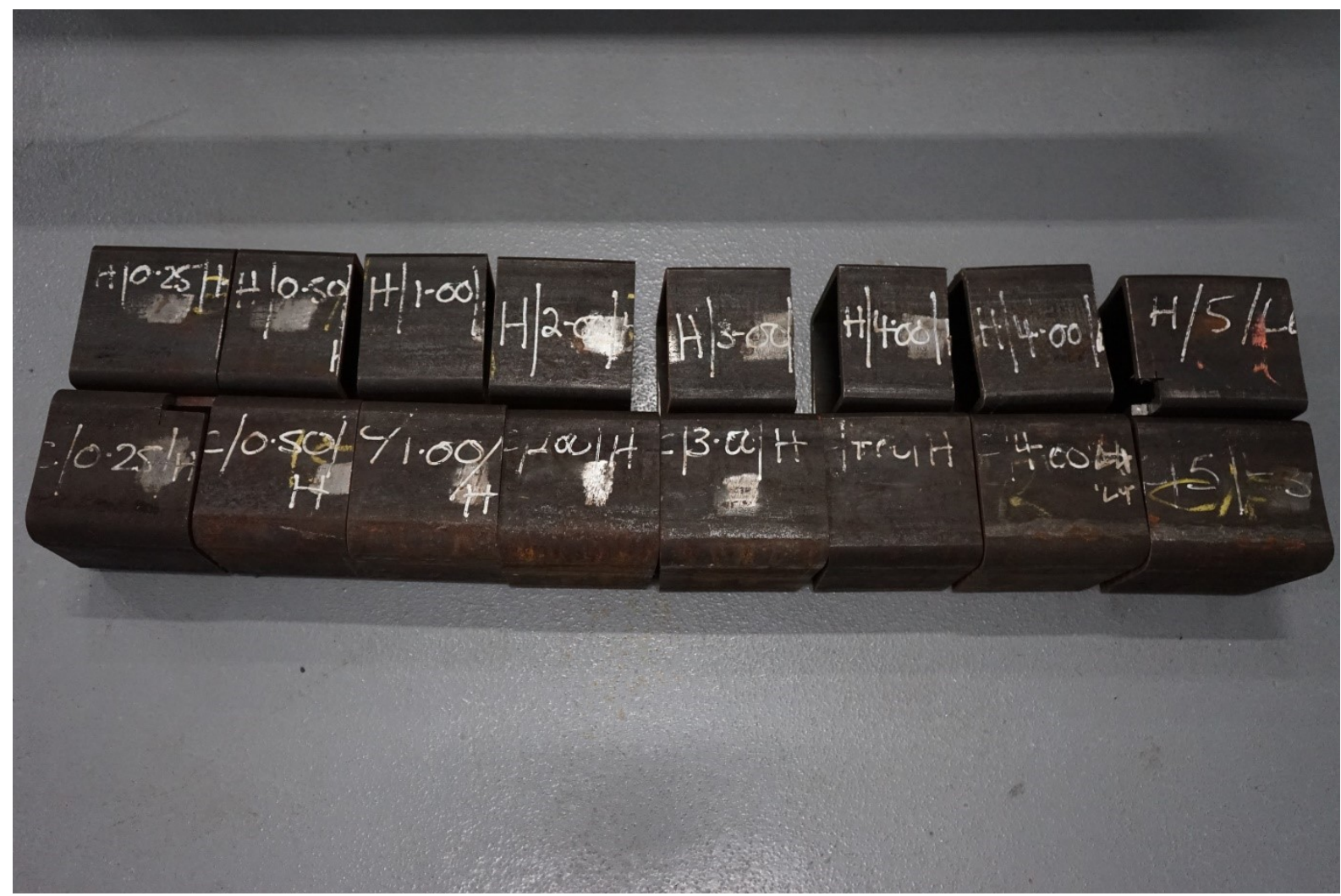

Figure 86-Hot-finished and cold-formed RHS sample lengths

Figure 86 is an image of the sample RHS used in the project for microstructure analysis and hardness testing. The samples were also used to determine the effect of bending on the dimensions of the sections including the wall thickness, out of square (height to width ratio) and corner radii.

Engineering design requires the use of a number of properties in order for correct material selection. These properties include mechanical properties such as the yield strength, the ultimate tensile strength, impact resistance, etc. [5], [7], [9], [55], [56] However a number of other properties are also required. These properties are derived from the dimensions of the section and include the second moment of area and the elastic modulus. The second moment of area is important as it indicates the resistance of a section to bending in a chosen direction with regards to its geometry.

It is therefore evident that the effect of manipulation by cold bending on the dimensions of the section is important. Alterations in dimensions will change the geometric properties, affecting the resistance of the section to further bending. The dimensions of the sections have been measured in order calculate the geometric properties. 


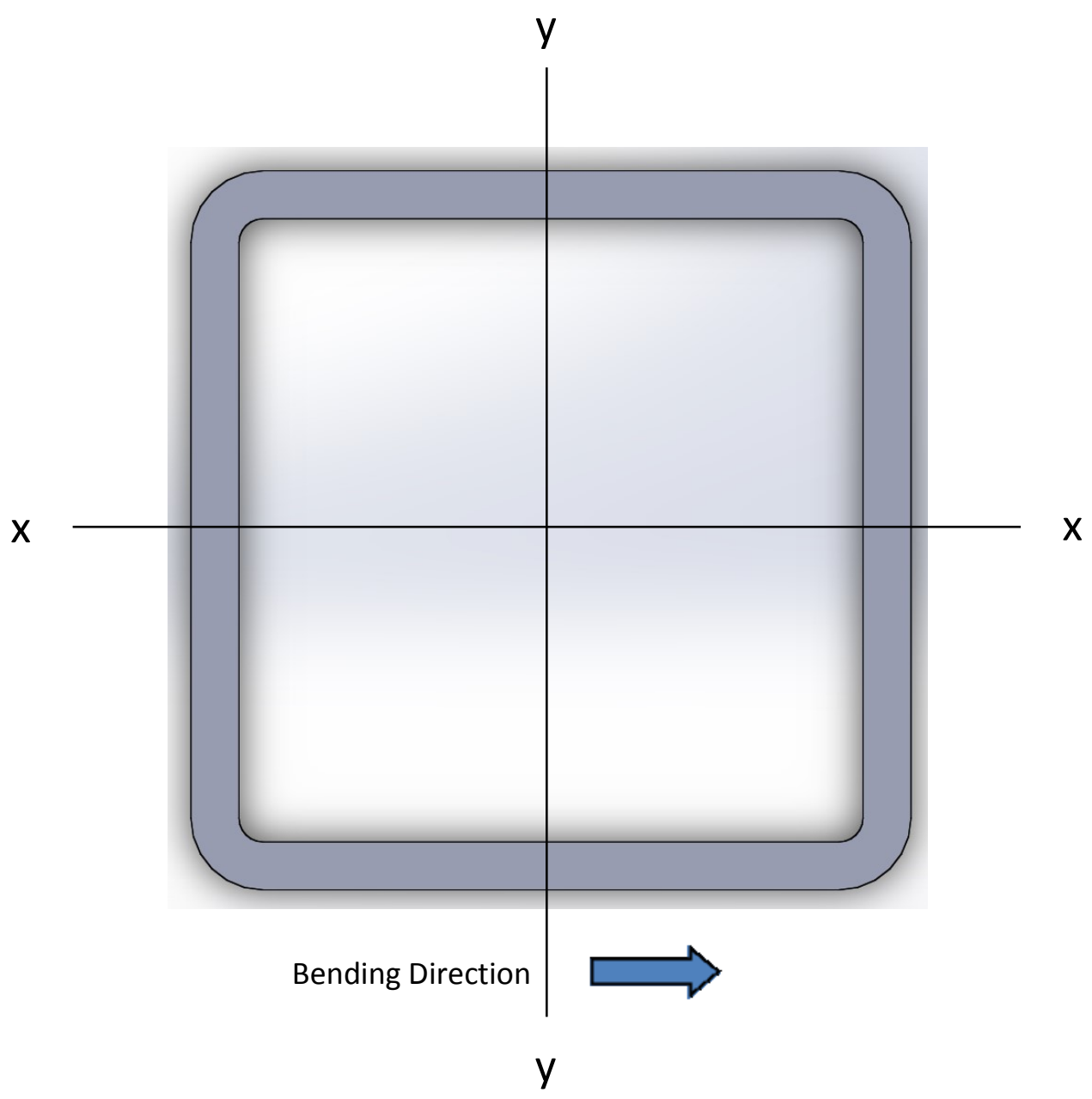

Figure 87-RHS Bending Direction and Axis 


\subsubsection{Wall Thickness}

\subsubsection{Hot-finished RHS Wall Thickness}

\begin{tabular}{|l|l|l|l|l|l|l|}
\hline & $\begin{array}{l}\text { Intrados } \\
(\mathrm{mm})\end{array}$ & $\begin{array}{l}\text { Intrados } \\
\text { deviation } \\
(\mathrm{mm})\end{array}$ & $\begin{array}{l}\text { Adjusted } \\
\text { Intrados } \\
\text { deviation } \\
(\mathrm{mm})\end{array}$ & $\begin{array}{l}\text { Extrados } \\
(\mathrm{mm})\end{array}$ & $\begin{array}{l}\text { Extrados } \\
\text { Deviation } \\
(\mathrm{mm})\end{array}$ & $\begin{array}{l}\text { Adjusted } \\
\text { Extrados } \\
\text { Deviation } \\
(\mathrm{mm})\end{array}$ \\
\hline $\mathbf{H / 0 . 0 0 / H}$ & 9.4 & -0.6 & 0 & 9.5 & -0.5 & 0 \\
\hline $\mathbf{H / 0 . 2 5 / H}$ & 9.5 & -0.5 & 0.1 & 9.4 & -0.6 & -0.1 \\
\hline $\mathbf{H / 0 . 5 0 / H}$ & 9.4 & -0.6 & 0 & 9.3 & -0.7 & -0.2 \\
\hline $\mathbf{H / 1 . 0 0 / H}$ & 9.5 & -0.5 & 0.1 & 9.4 & -0.6 & -0.1 \\
\hline $\mathbf{H / 2 . 0 0 / H}$ & 9.5 & -0.5 & 0.1 & 9.4 & -0.6 & -0.1 \\
\hline $\mathbf{H / 3 . 0 0 / H}$ & 9.6 & -0.4 & 0.2 & 9.4 & -0.6 & -0.1 \\
\hline $\mathbf{H / 4 . 0 0 H}$ & 9.6 & -0.4 & 0.2 & 9.4 & -0.6 & -0.1 \\
\hline $\mathbf{H / 4 . 0 0 / L 6}$ & 9.5 & -0.5 & 0.1 & 9.3 & -0.7 & -0.2 \\
\hline $\mathbf{H / 5 . 0 0 / L 6}$ & 9.6 & -0.4 & 0.2 & 9.4 & -0.6 & -0.1 \\
\hline
\end{tabular}

Table 11-Table showing the wall thickness for hot-finished rectangular hollow section samples 


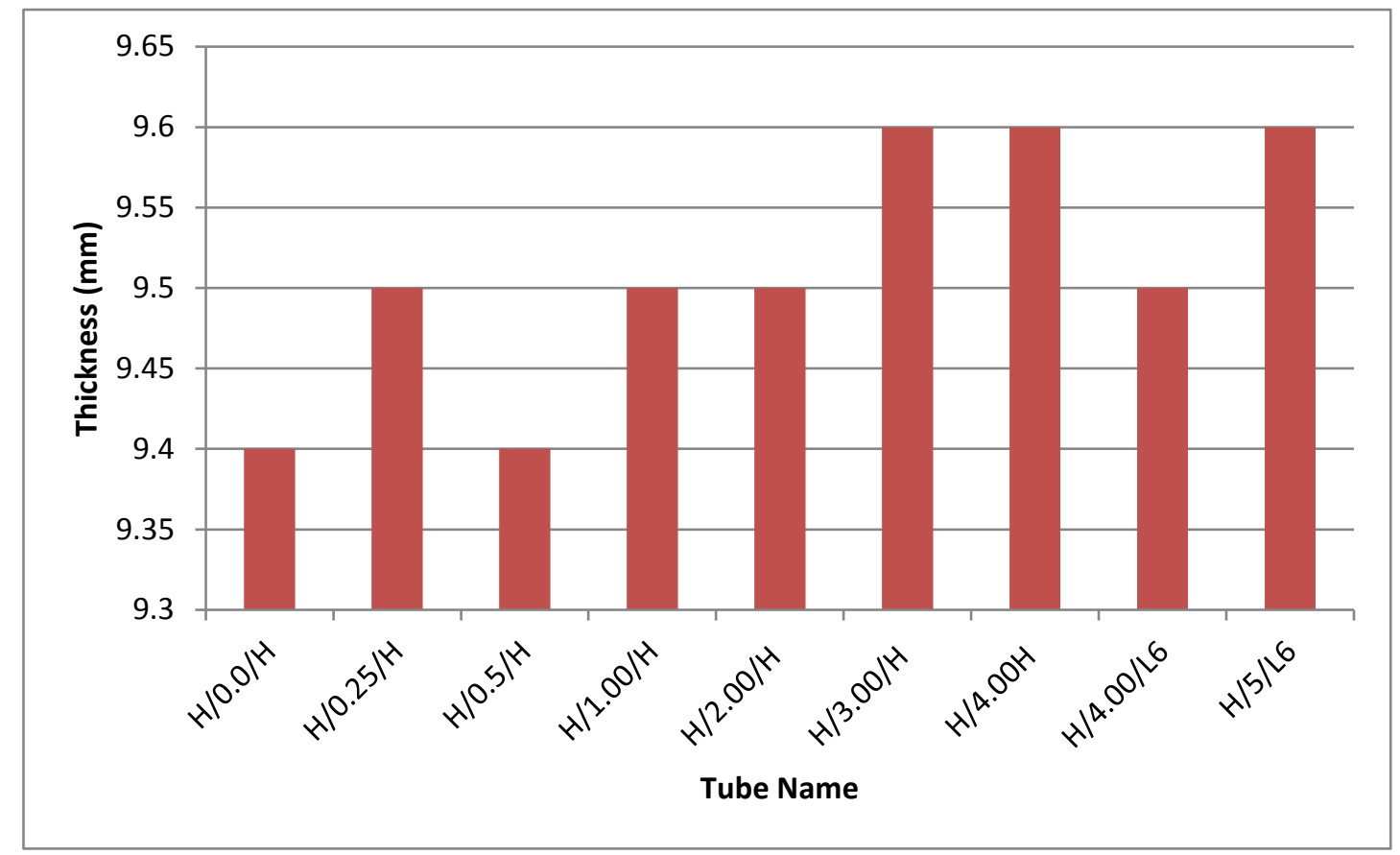

Figure 88-Wall thickness at the hot finished intrados

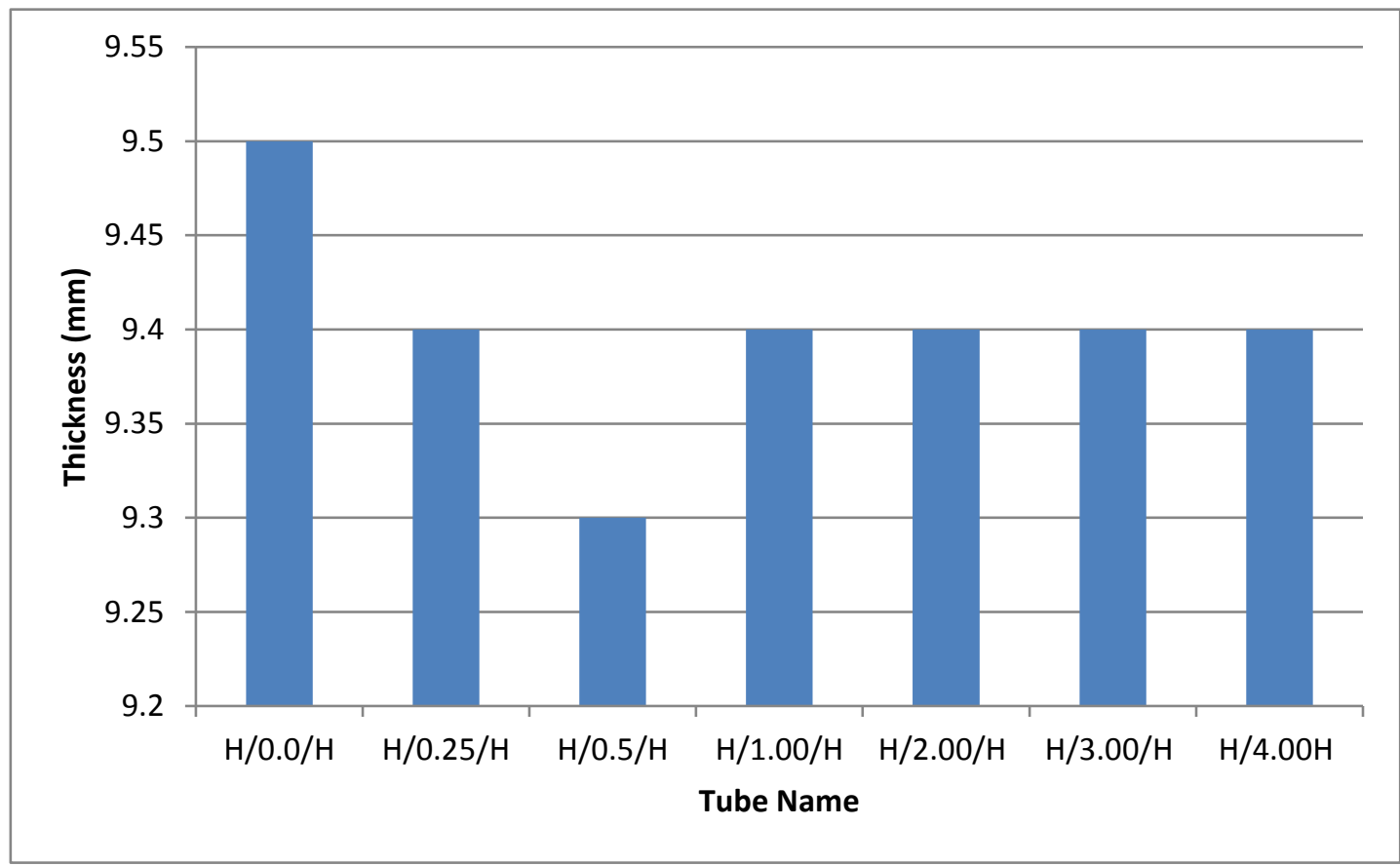

Figure 89-Wall thickness at the hot-finished extrados

The failure criterion for an unbent hot-finished RHS in BS EN 10210 [55], [56] is -10\% of the wall thickness. This equates to a wall thickness of anything less than $9 \mathrm{~mm}$. Bent sections are not required to meet this specification; however all of the wall thickness measurements lie within the specification limits. 


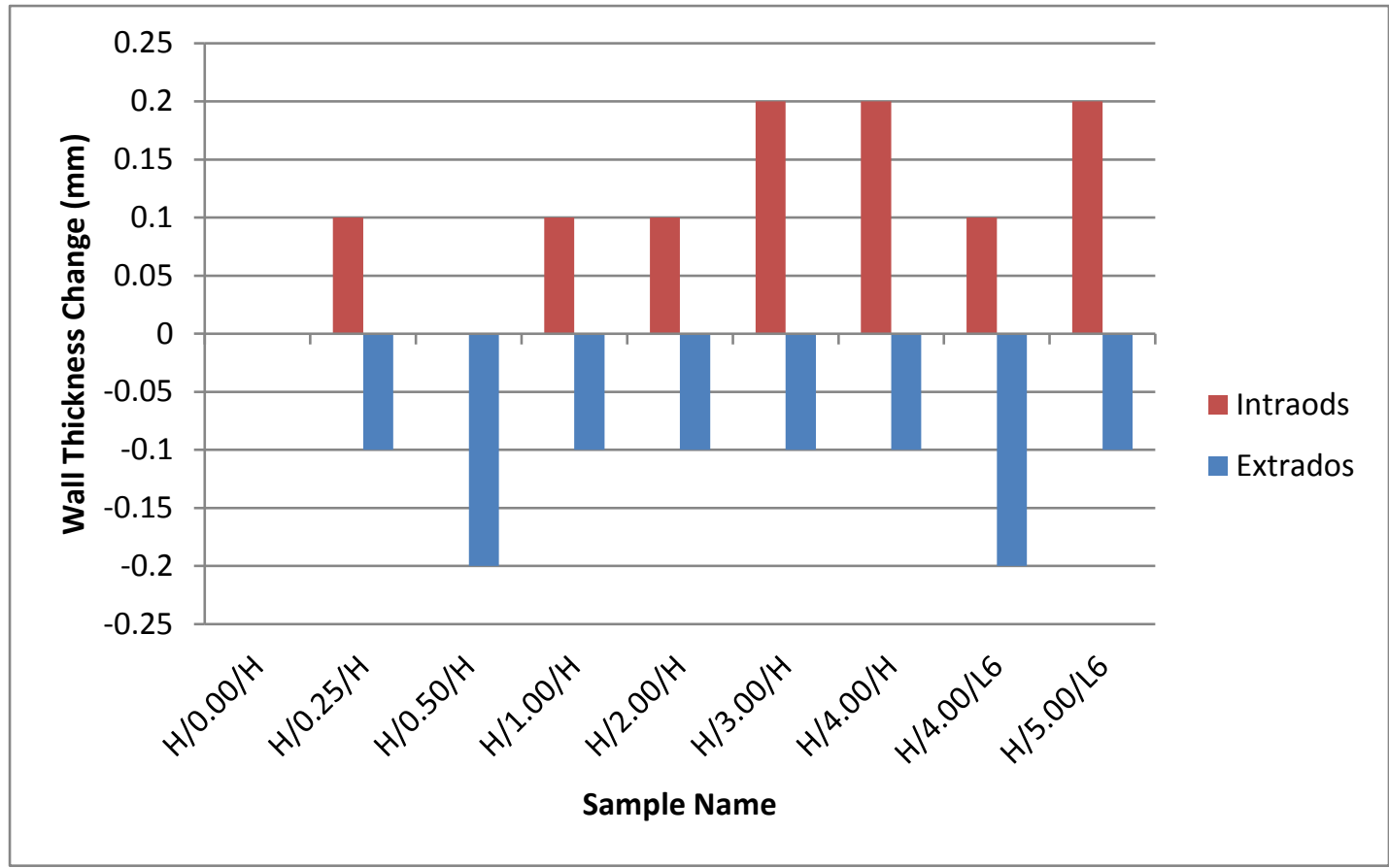

Figure 90-Adjusted Wall Thickness Deviation of Hot-finished RHS

It is evident that the number of times that the section is passed through the forming rollers has an effect on the wall thickness of the section at the intrados and the extrados. It can be seen in Figure 90 that at the intrados, reducing the number of passes reduces the effect of wall thickening. However, reducing the number of passes increases the wall thinning at the extrados at $4 \%$ strain. 
6.6.1.2. Cold-formed RHS Wall Thickness

\begin{tabular}{|c|c|c|c|c|c|c|}
\hline & $\begin{array}{l}\text { Intrados } \\
(\mathrm{mm})\end{array}$ & $\begin{array}{l}\text { Intrados } \\
\text { deviation } \\
(\mathrm{mm})\end{array}$ & $\begin{array}{l}\text { Adjusted } \\
\text { Intrados } \\
\text { deviation } \\
\text { (mm) }\end{array}$ & $\begin{array}{l}\text { Extrados } \\
(\mathrm{mm})\end{array}$ & $\begin{array}{l}\text { Extrados } \\
\text { Deviation } \\
(\mathrm{mm})\end{array}$ & $\begin{array}{l}\text { Adjusted } \\
\text { Extrados } \\
\text { Deviation } \\
(\mathrm{mm})\end{array}$ \\
\hline $\mathrm{C} / 0.00 / \mathrm{H}$ & 10.1 & 0.1 & 0 & 10 & 0 & 0 \\
\hline $\mathrm{C} / 0.25 / \mathrm{H}$ & 10 & 0 & -0.1 & 10.1 & 0.1 & 0.1 \\
\hline $\mathrm{C} / 0.50 / \mathrm{H}$ & 10 & 0 & -0.1 & 10.1 & 0.1 & 0.1 \\
\hline $\mathrm{C} / 1.00 / \mathrm{H}$ & 10 & 0 & -0.1 & 10 & 0 & 0 \\
\hline $\mathrm{C} / 2.00 / \mathrm{H}$ & 10.1 & 0.1 & 0 & 9.8 & -0.2 & -0.2 \\
\hline $\mathrm{C} / 3.00 / \mathrm{H}$ & 10.1 & 0.1 & 0 & 9.8 & -0.2 & -0.2 \\
\hline $\mathrm{C} / 4.00 / \mathrm{H}$ & 10.1 & 0.1 & 0 & 9.8 & -0.2 & -0.2 \\
\hline $\mathrm{C} / 4.00 / \mathrm{L} 4$ & 10.2 & 0.2 & 0.1 & 9.8 & -0.2 & -0.2 \\
\hline C/5.00/L3 & 10.2 & 0.2 & 0.1 & 9.7 & -0.3 & -0.3 \\
\hline
\end{tabular}

Table 12-Table showing the wall thickness for cold-formed rectangular hollow section samples

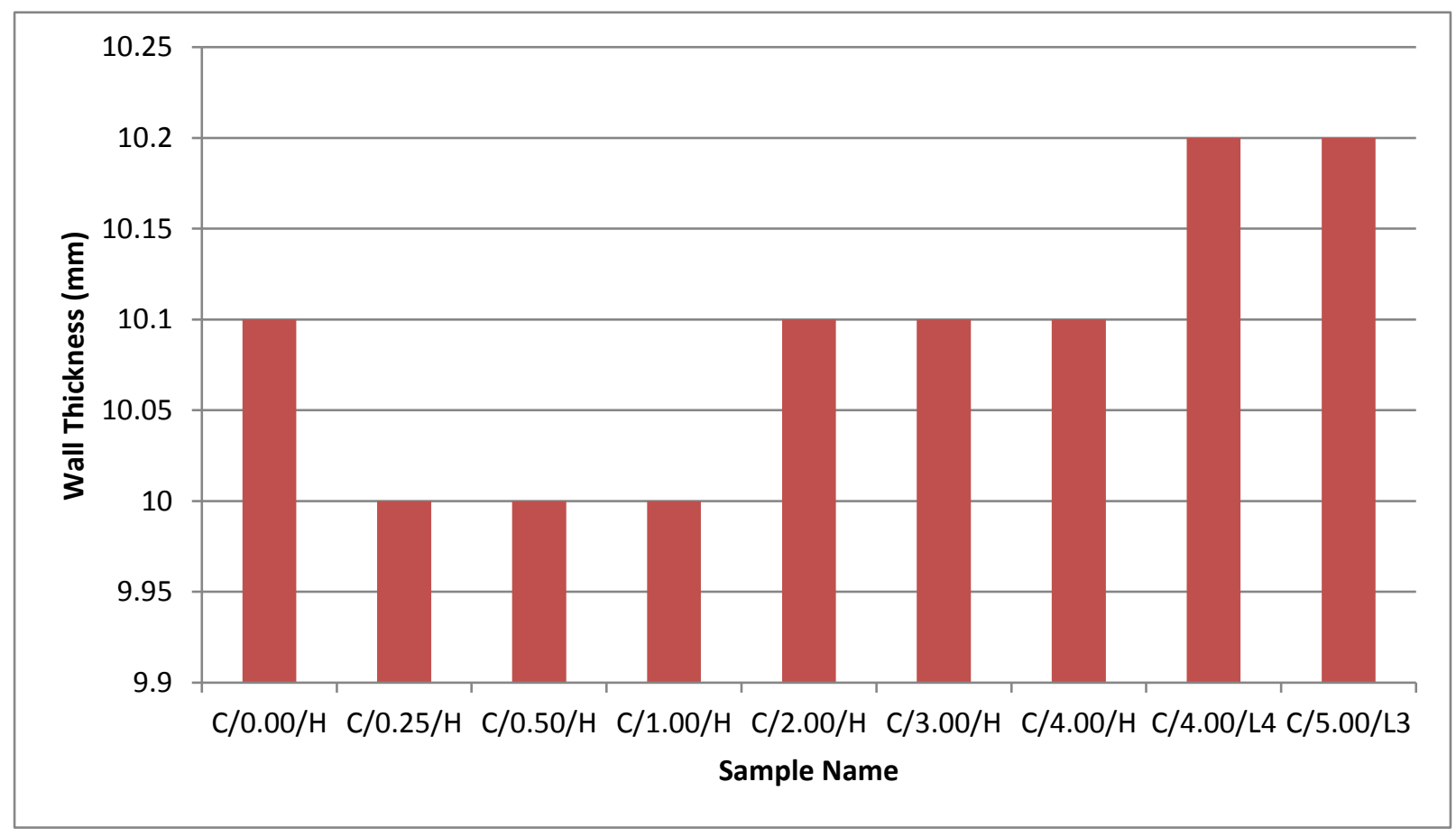

Figure 91-Cold-formed RHS Intrados wall thickness 


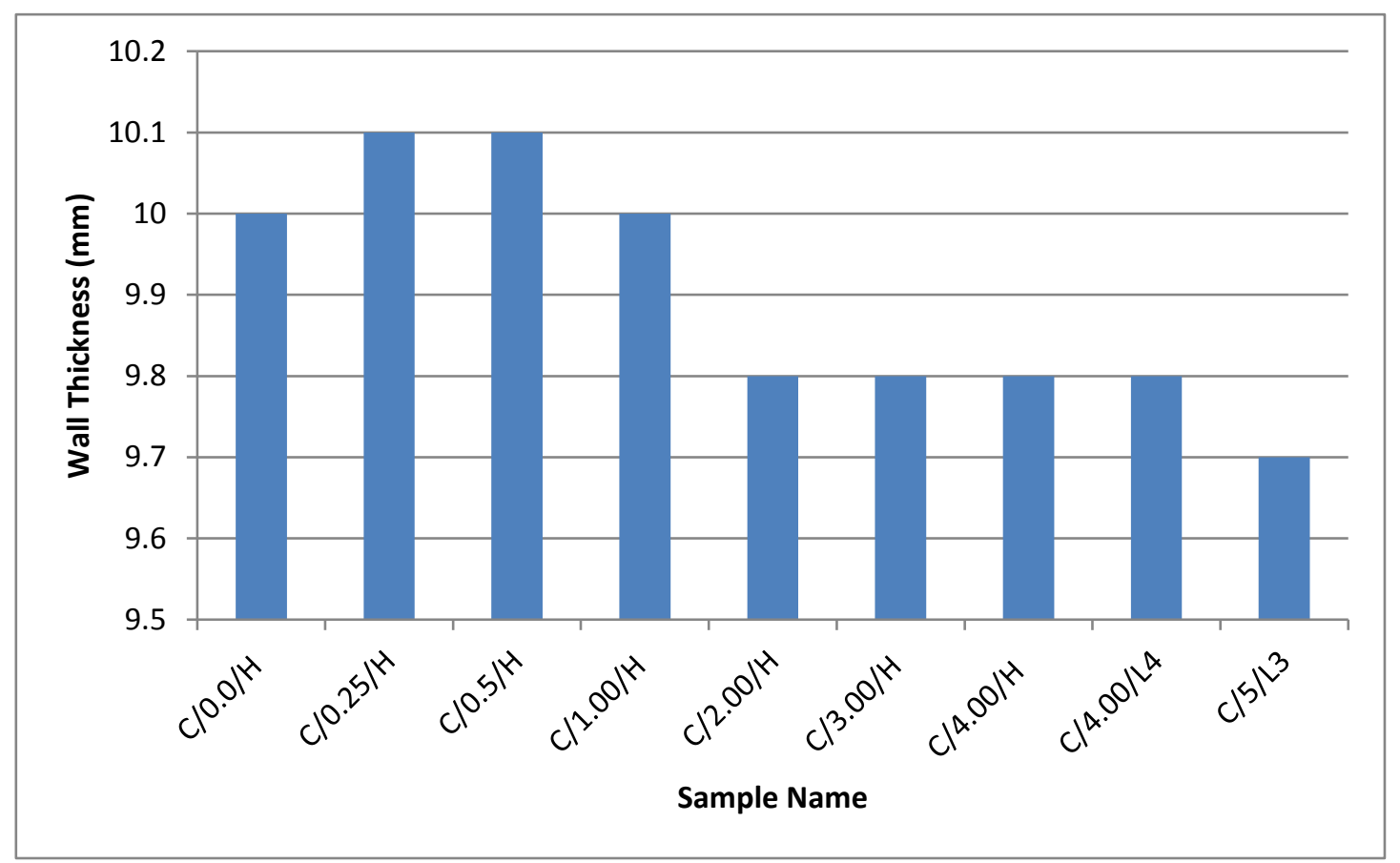

Figure 92-Cold-formed RHS extrados wall thickness

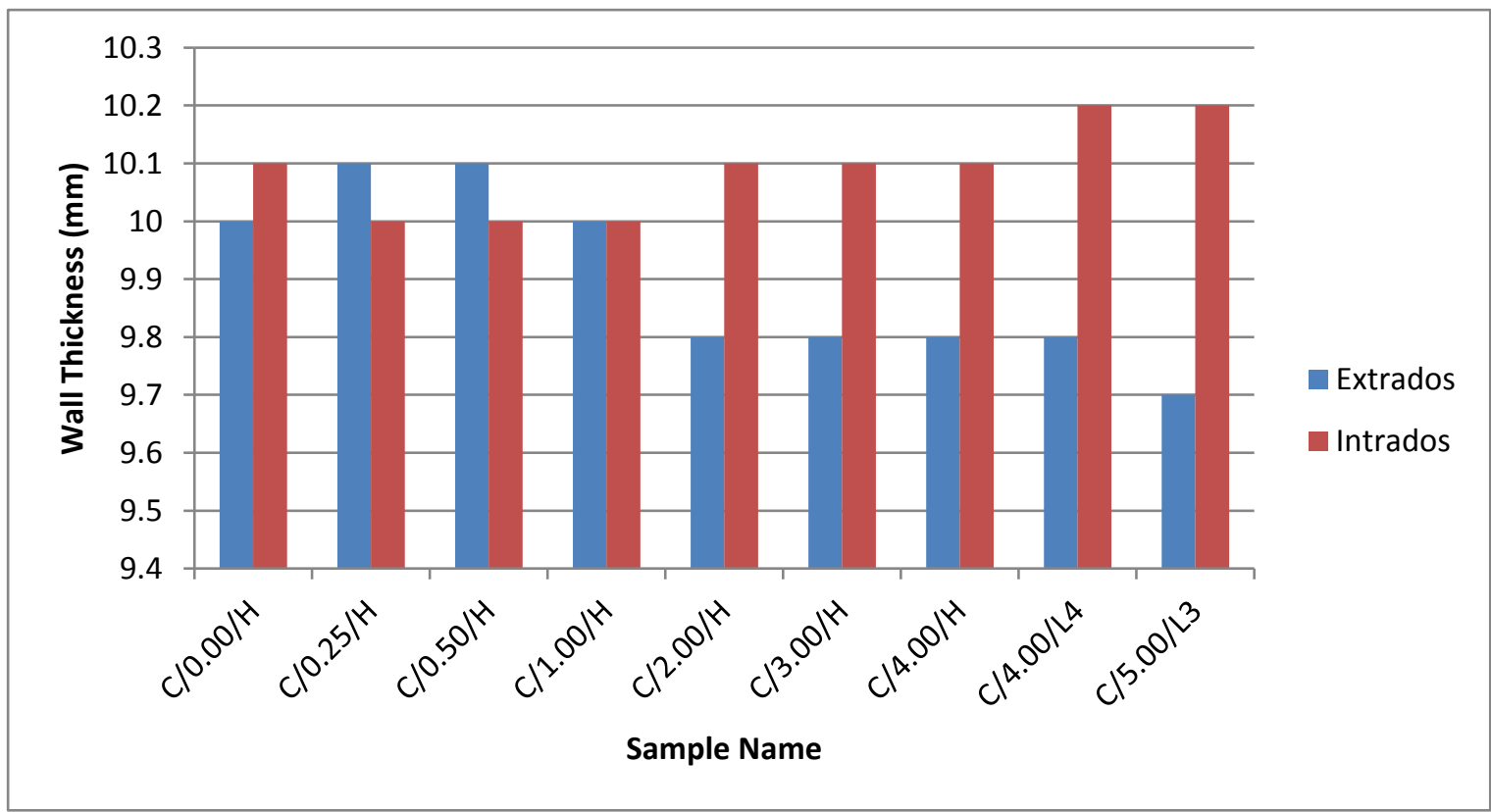

Figure 93-Wall Thickness of the Cold-formed RHS 


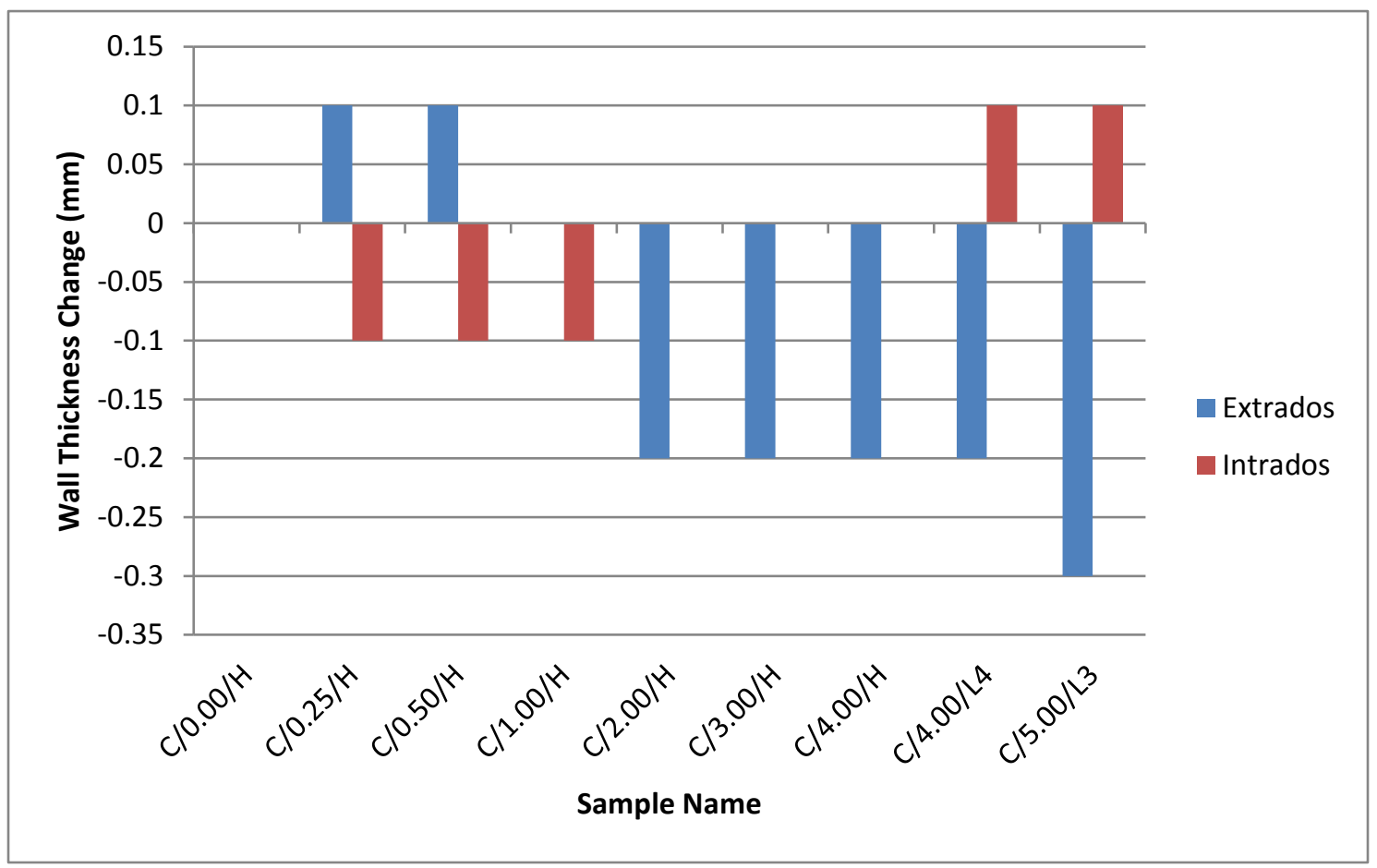

Figure 94-Wall Thickness Change of the Cold-formed RHS

The failure criterion for an unbent cold-formed RHS in BS EN 10219 is $\pm 0.5 \mathrm{~mm}$ of the wall thickness[7], [9]. This equates to a wall thickness of anything less than $9.5 \mathrm{~mm}$ for a $10 \mathrm{~mm}$ thick wall. Bent sections are not required to meet this specification; however all of the wall thickness measurements lie within the specification limits. 


\subsubsection{Out of square}

\subsubsection{Hot-finished RHS Out of square}

\begin{tabular}{|l|l|l|l|}
\hline & Depth $(\mathrm{mm})$ & Breadth $(\mathrm{mm})$ & Out of square (\%) \\
\hline H/0.00/H & 150.0 & 149.7 & 0.2 \\
\hline H/0.25/H & 151.5 & 146.7 & 3.2 \\
\hline H/0.50/H & 152.3 & 145.4 & 4.6 \\
\hline H/1.00/H & 152.1 & 143.7 & 5.7 \\
\hline H/2.00/H & 152.7 & 142.4 & 7.0 \\
\hline H/3.00/H & 152.8 & 141.0 & 8.1 \\
\hline H/4.00/H & 153.0 & 139.9 & 8.9 \\
\hline H/4.00/L6 & 153.0 & 141.6 & 7.7 \\
\hline H/5.00/L6 & 152.5 & 141.3 & 7.6 \\
\hline
\end{tabular}

Table 13-Hot-finished RHS out of square

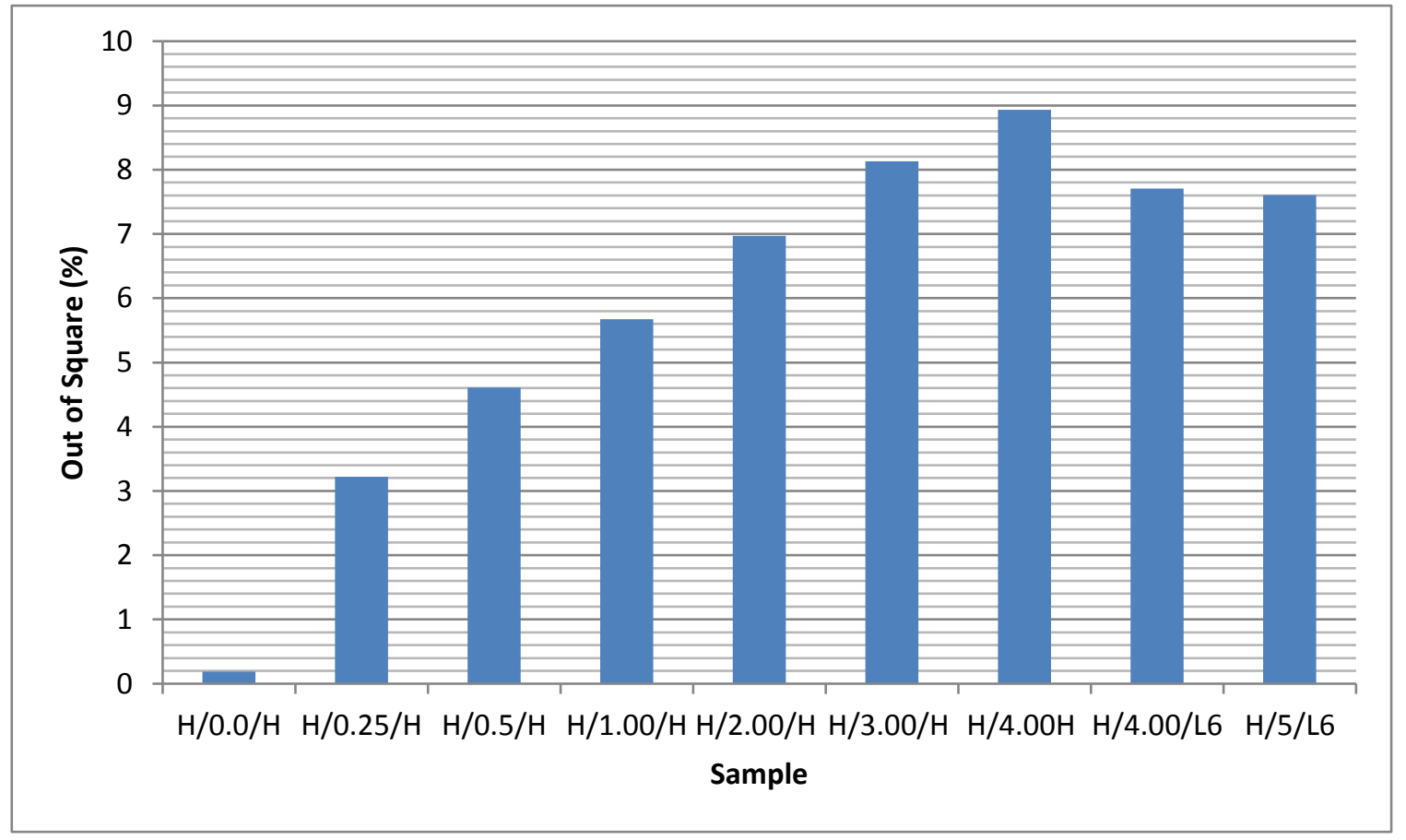

Figure 95-Hot-finished RHS out of square 
It can be seen from Figure 95 that the percentage out of square increased as strain was increased. However, this increase is not proportional to strain applied. A rapid increase was observed initially before the rate of increase slowed. Reducing the number of passes though the forming rolls decreases the effect on the depth to breadth ratio.

The failure criterion for an unbent hot-finished RHS in BS EN 10210 [55], [56] is $\pm 1 \%$ of the specified outer dimensions in either depth or the breadth. This equates to an allowable wall length of anything between the limits $148.5 \mathrm{~mm}<\mathrm{T}>151.5 \mathrm{~mm}$ for a $150 \times 150 \times 10$ RHS. Bent sections are not required to meet this specification; however, all of the bent sections would fail this specification due to the deformation caused by bending. 


\subsubsection{Cold-formed RHS Out of square}

\begin{tabular}{|llll|} 
& Depth $(\mathrm{mm})$ & Breadth $(\mathrm{mm})$ & Out of square (\%) \\
\hline C/0.00/H & 150.8 & 151.0 & -0.1 \\
\hline C/0.25/H & 152.6 & 148.1 & 3.0 \\
\hline C/0.50/H & 152.8 & 144.9 & 5.3 \\
\hline C/1.00/H & 152.9 & 143.4 & 6.4 \\
\hline C/2.00/H & 153.1 & 140.9 & 8.3 \\
\hline C/3.00/H & 153.2 & 137.2 & 11.0 \\
\hline C/4.00/H & 152.8 & 135.9 & 11.7 \\
\hline C/4.00/L4 & 153.3 & 139.5 & 9.4 \\
\hline C/5.00/L3 & 153.6 & 136.6 & 11.7 \\
\hline
\end{tabular}

Table 14-Cold-formed RHS out of round

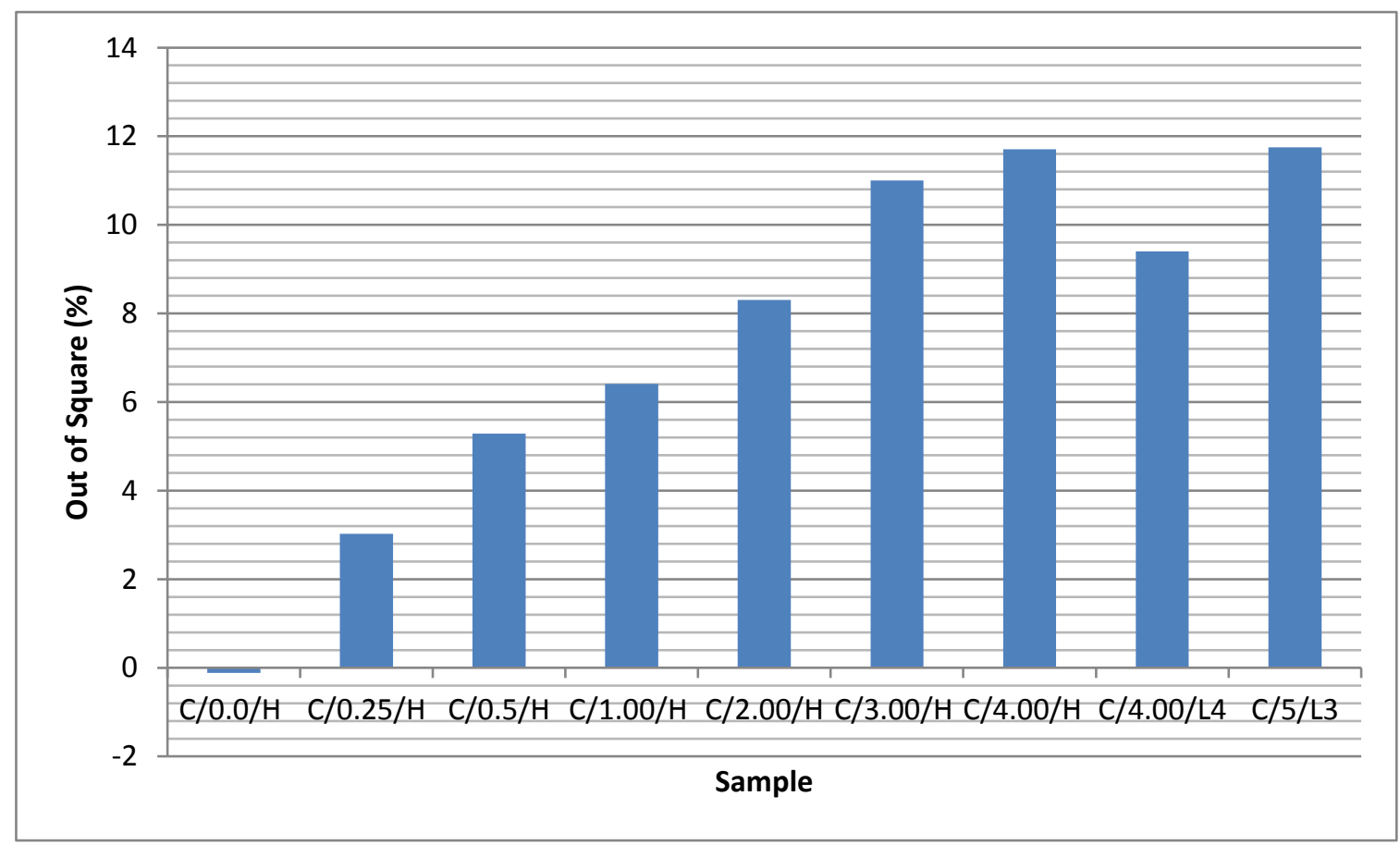

Figure 96-Cold-formed RHS out of square 
It can be seen from Figure 96 that the out of round ratio increased as strain was increased. Reducing the number of passes though the forming rolls decreases the effect on the depth to breadth ratio. The failure criterion for an unbent cold-formed RHS in BS EN 10219 is $\pm 0.8 \%$ of the specified outer dimensions in either depth or the breadth[7], [9]. This equates to an allowable wall length of anything between the limits $148.8 \mathrm{~mm}<T>151.2 \mathrm{~mm}$ for a $150 \times 150 \times 10$ RHS. Bent sections are not required to meet this specification; however, all of the sections would fail this specification due to the deformation caused by bending. The cold-formed material has become more distorted by the bending process than the hot-finished material. 


\subsubsection{Corner Radii}

\subsubsection{Hot-finished RHS Corner Radii}

\begin{tabular}{|c|c|c|c|}
\hline & Before $(\mathrm{mm})$ & After (mm) & $\begin{array}{l}\text { Difference } \\
(\mathrm{mm})\end{array}$ \\
\hline$H / 0.00 / H$ & 16 & 16 & 0 \\
\hline $\mathrm{H} / 0.25 / \mathrm{H}$ & 16 & 15 & -1 \\
\hline $\mathrm{H} / 0.50 / \mathrm{H}$ & 16 & 15 & -1 \\
\hline$H / 1.00 / H$ & 16 & 14 & -2 \\
\hline$H / 2.00 / H$ & 16 & 13 & -3 \\
\hline$H / 3.00 / H$ & 16 & 13 & -3 \\
\hline$H / 4.00 / H$ & 16 & 12 & -4 \\
\hline H/4.00/L6 & 16 & 12 & -4 \\
\hline H/5.00/L6 & 16 & 12 & -4 \\
\hline
\end{tabular}

Table 15-Hot-finished RHS Corner Radii

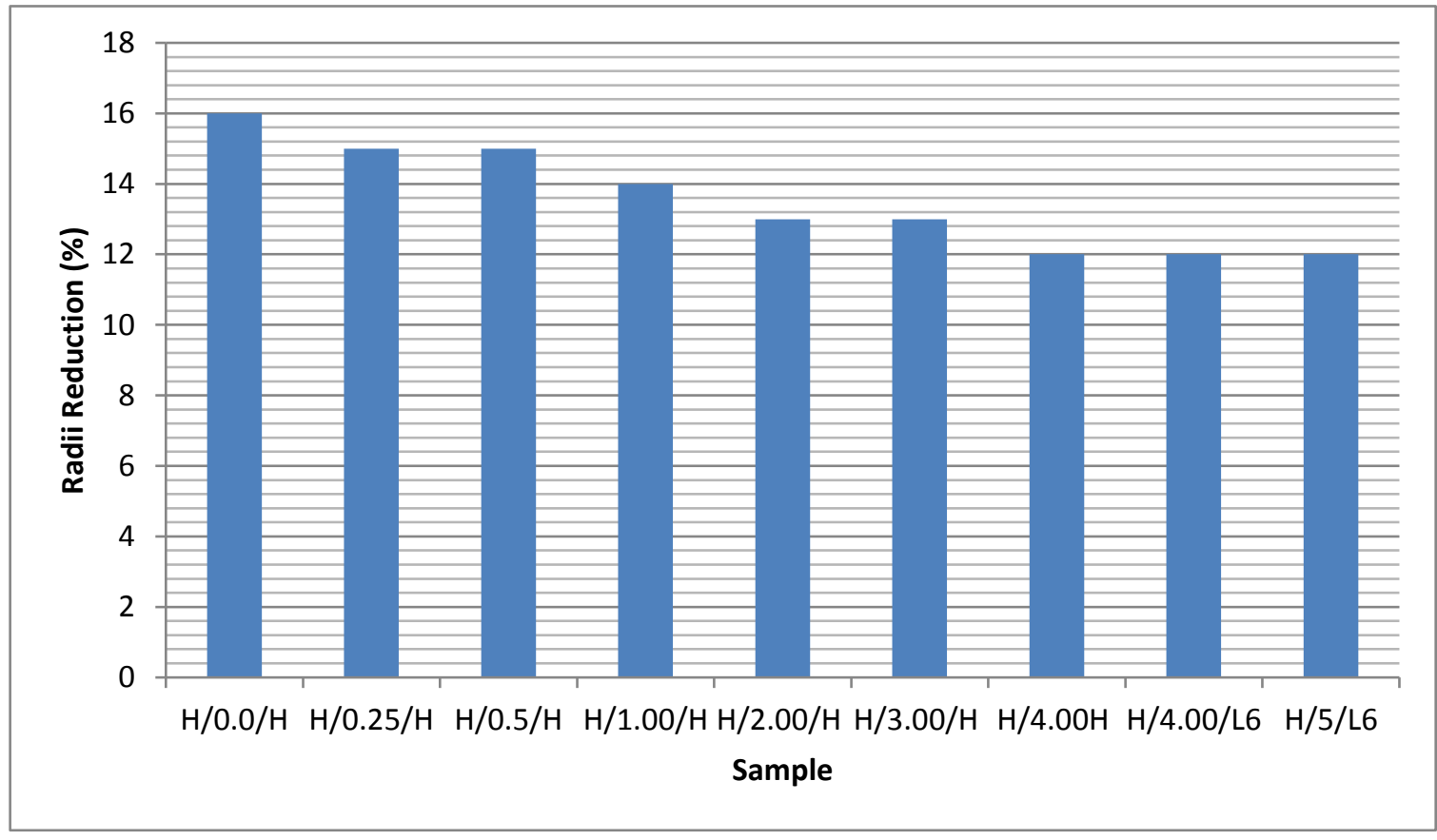

Figure 97-Hot-finished RHS percentage reduction in intrados corner radii 
The failure criterion for an unbent hot-finished RHS in BS EN 10210 is $\leq 3 T$ [55], [56]. This equates to an allowable corner radius of anything $\leq 30 \mathrm{~mm}$ for a $10 \mathrm{~mm}$ gauge RHS. Bent sections are not required to meet this specification; however, all of the samples lie within specification.

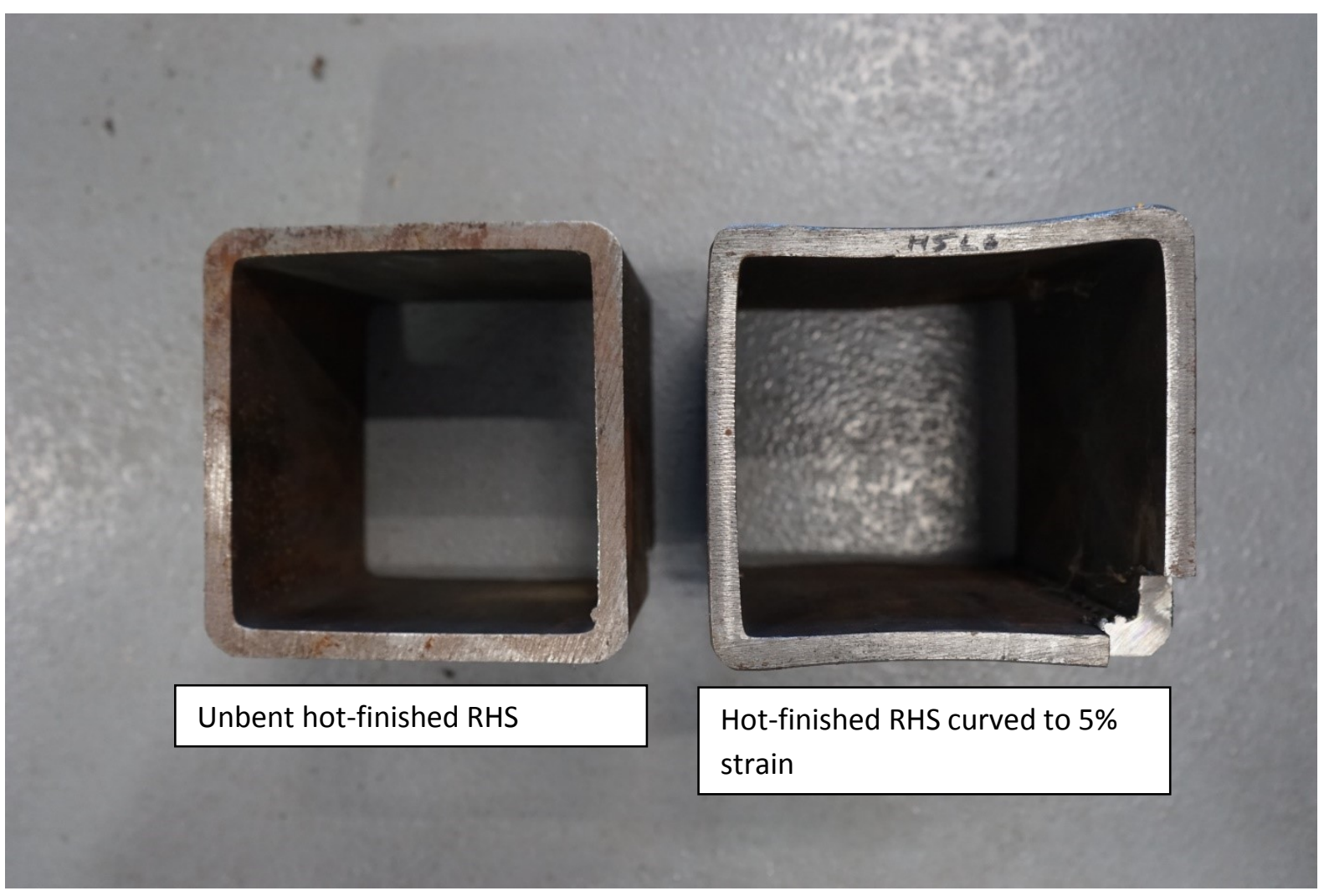

Figure 98-Hot-finished RHS low v high strain corner radii 
6.6.3.2. Cold-formed RHS Corner Radii

\begin{tabular}{|llll|}
\hline Sample & $\begin{array}{l}\text { Before } \\
(\mathrm{mm})\end{array}$ & $\begin{array}{l}\text { After } \\
(\mathrm{mm})\end{array}$ & $\begin{array}{l}\text { Difference } \\
(\mathrm{mm})\end{array}$ \\
\hline C/0.0/H & 25 & 25 & 0 \\
\hline C/0.25/H & 25 & 25 & 0 \\
\hline C/0.50/H & 25 & 24 & -1 \\
\hline C/1.00/H & 25 & 23 & -2 \\
\hline C/2.00/H & 25 & 23 & -2 \\
\hline C/3.00/H & 25 & 22 & -3 \\
\hline C/4.00/H & 25 & 21 & -4 \\
\hline C/4.00/L4 & 25 & 23 & -2 \\
\hline C/5.00/L3 & 25 & 22 & -3 \\
\hline
\end{tabular}

Table 16-Cold-formed RHS Corner Radii

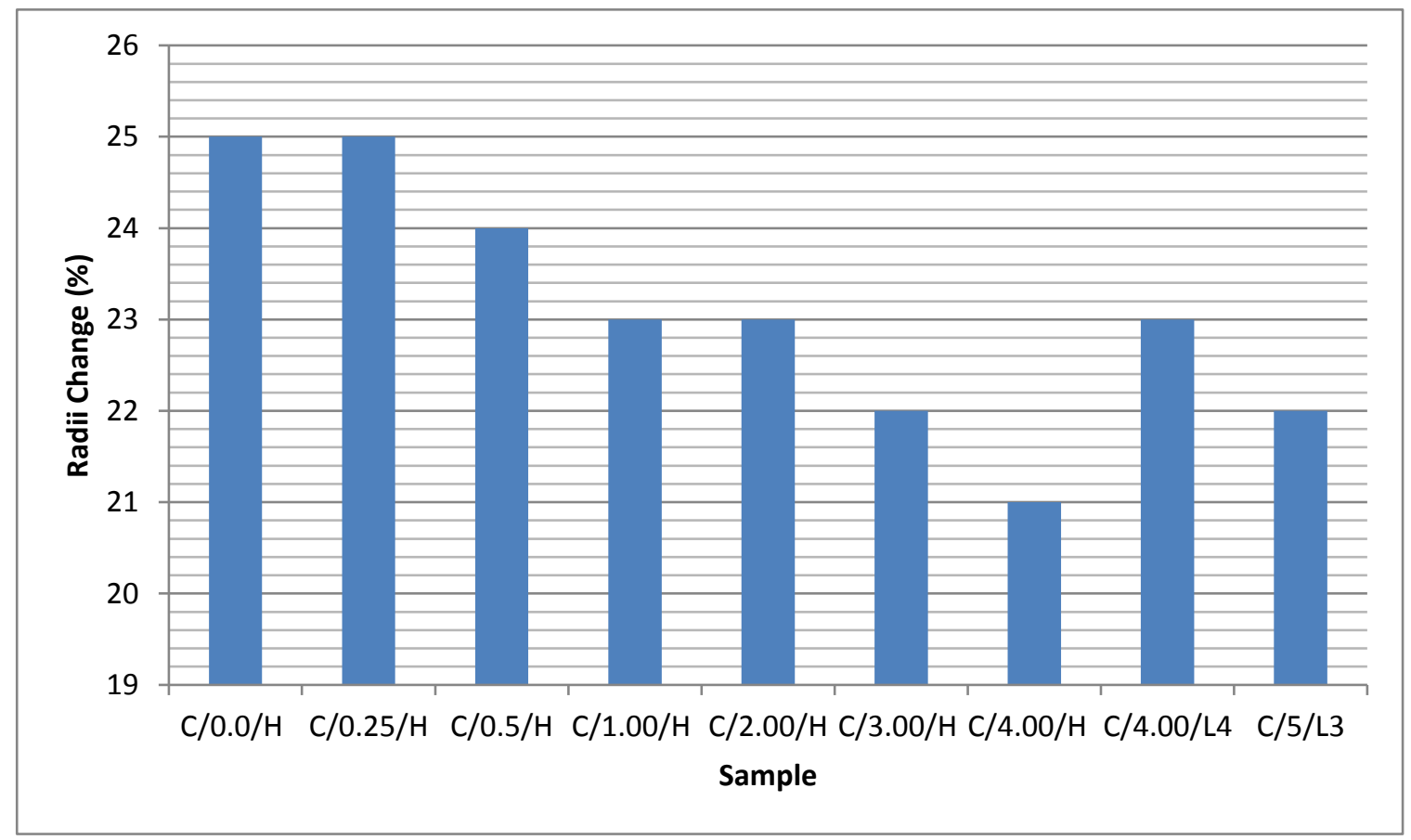

Figure 99-Cold-formed RHS Percentage Intrados Corner Radii Reduction 


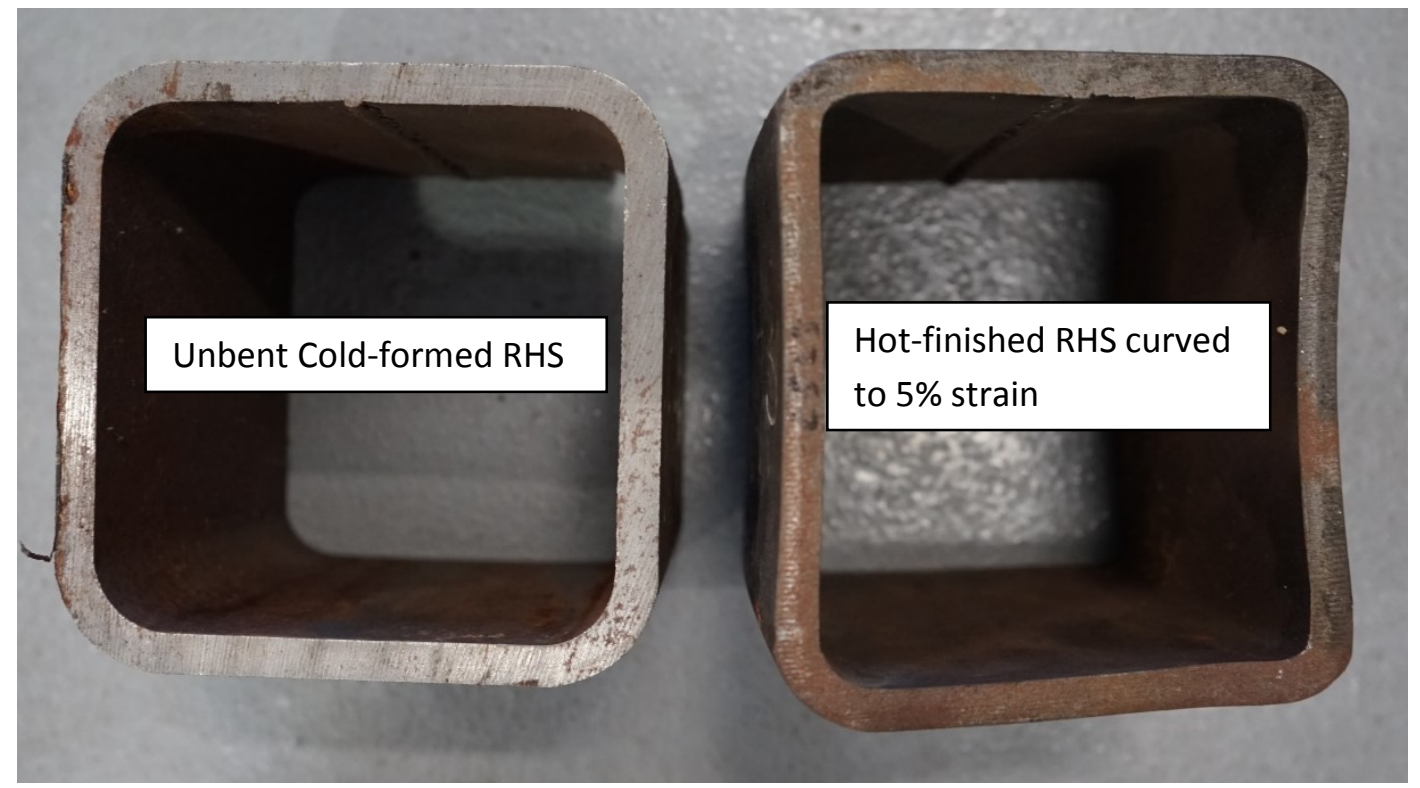

Figure 100-Cold-formed RHS low v high strain corner radii

The failure criterion for an unbent cold-formed RHS in BS EN 10219 is between 2Tand 3T[7], [9]. This equates to an allowable corner radius of anything between the limits $20 \mathrm{~mm}<\mathrm{T}>30 \mathrm{~mm}$ for a $10 \mathrm{~mm}$ gauge RHS. Bent sections are not required to meet this specification; however, all of the samples lie within specification.

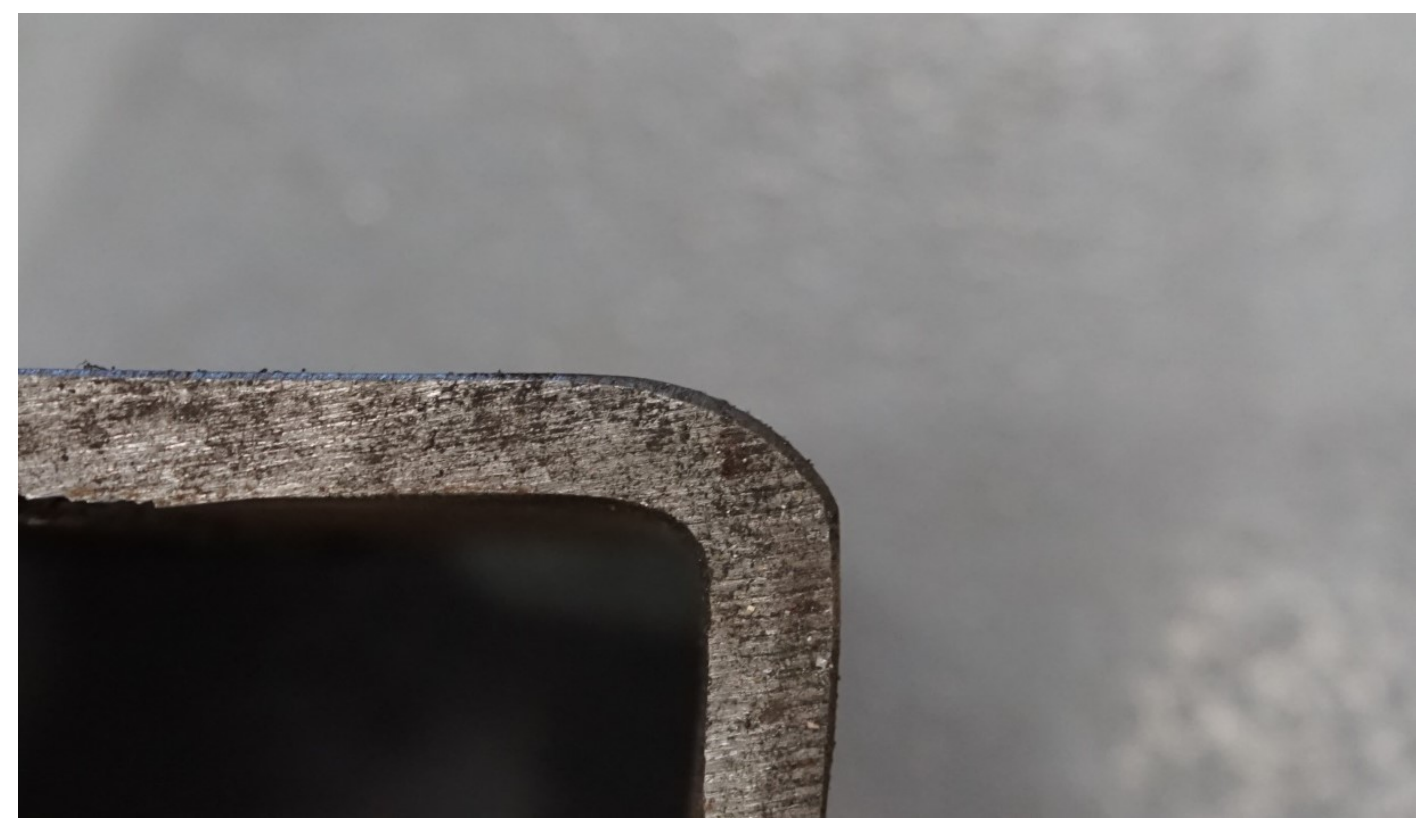

Figure 101-Intrados corner profile of a high strain cold-formed RHS 


\subsubsection{Concavity}

\subsubsection{Hot-finished RHS Wall Concavity}

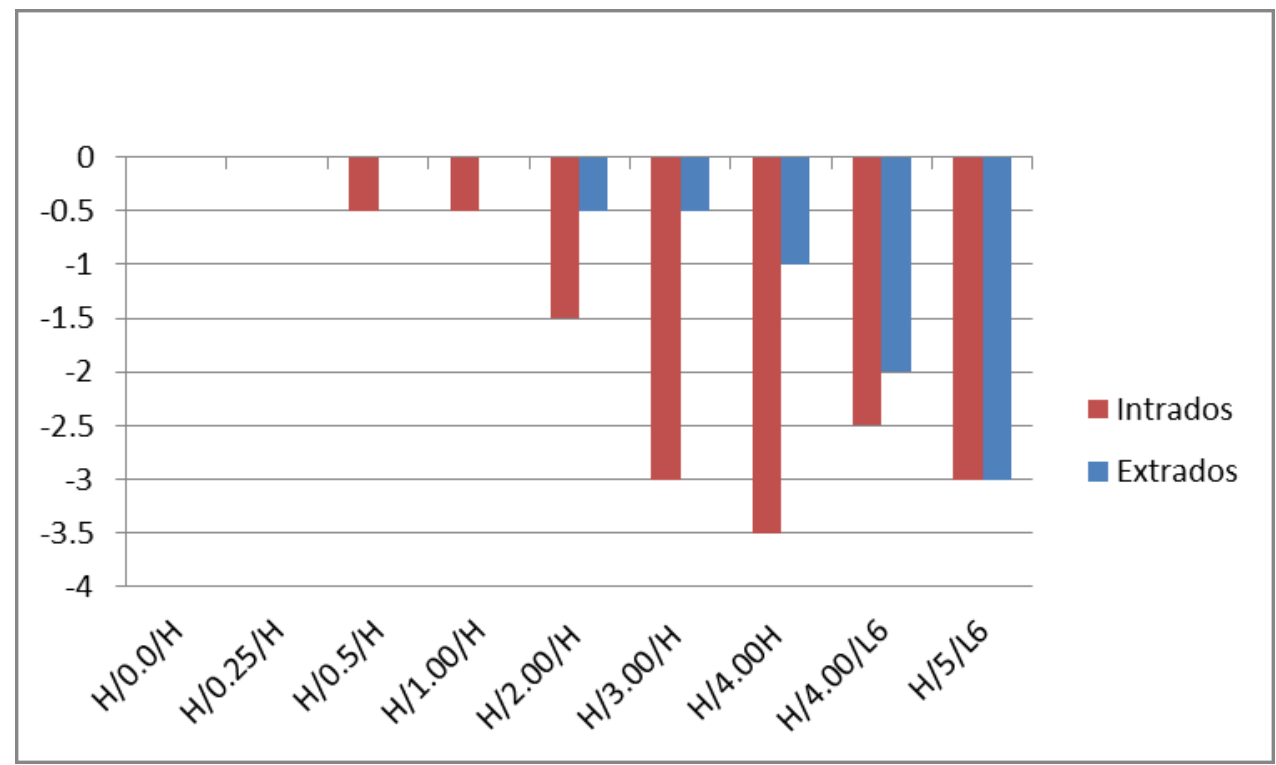

Figure 102-Hot-finished RHS Wall Deflection

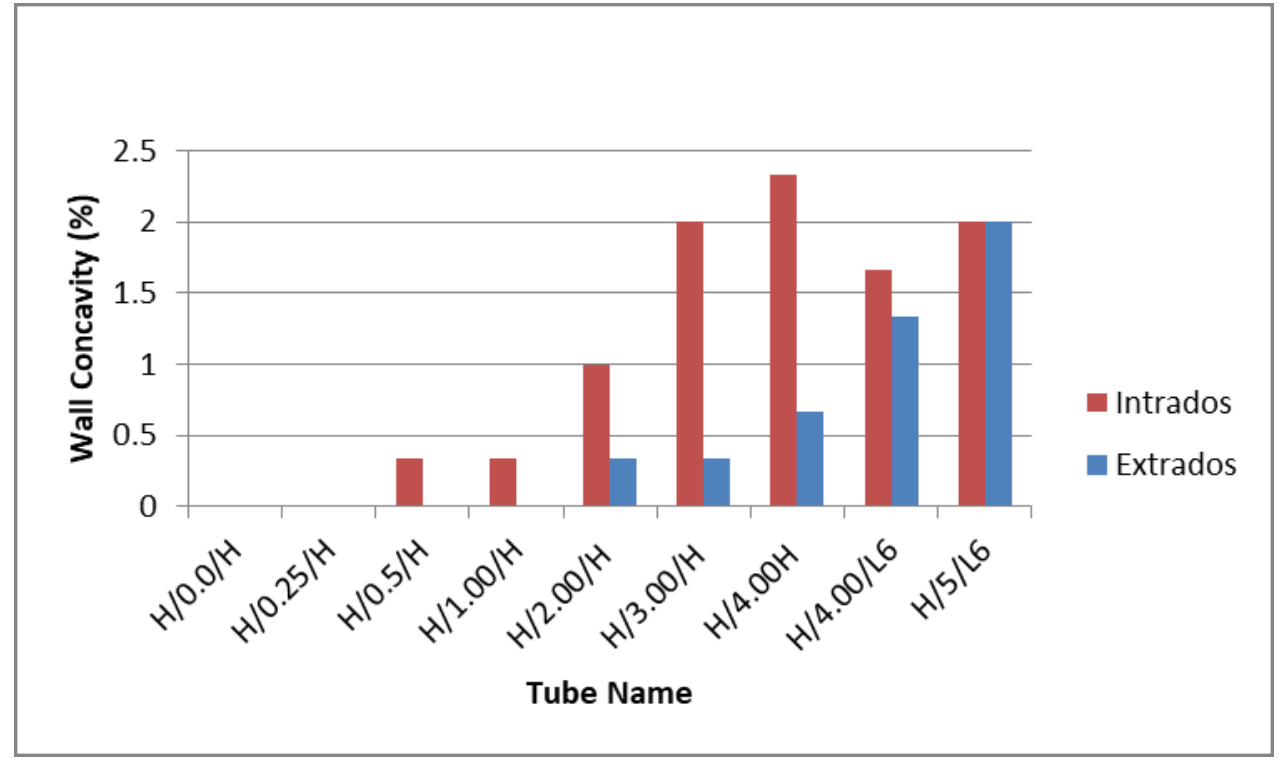

Figure 103-Hot-finished RHS Wall Concavity 


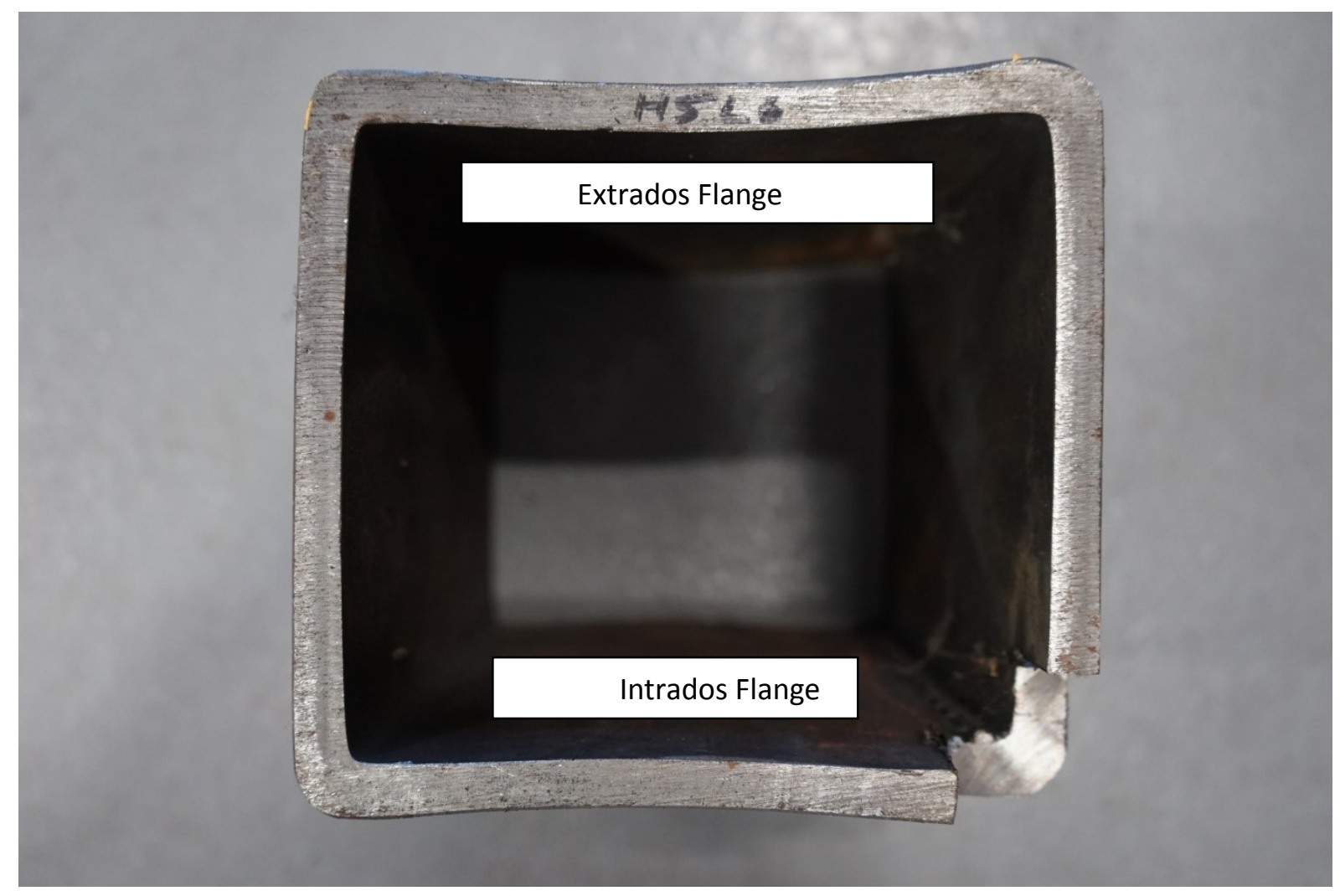

\section{Figure 104-Intrados concavity of hot-finished RHS}

Figure 104 is an example of the concavity brought about by the bending of the section. Both the intrados and extrados (bottom and top respectively) underwent wall collapse due to bending. 
6.6.4.2. Cold-formed RHS Wall Concavity

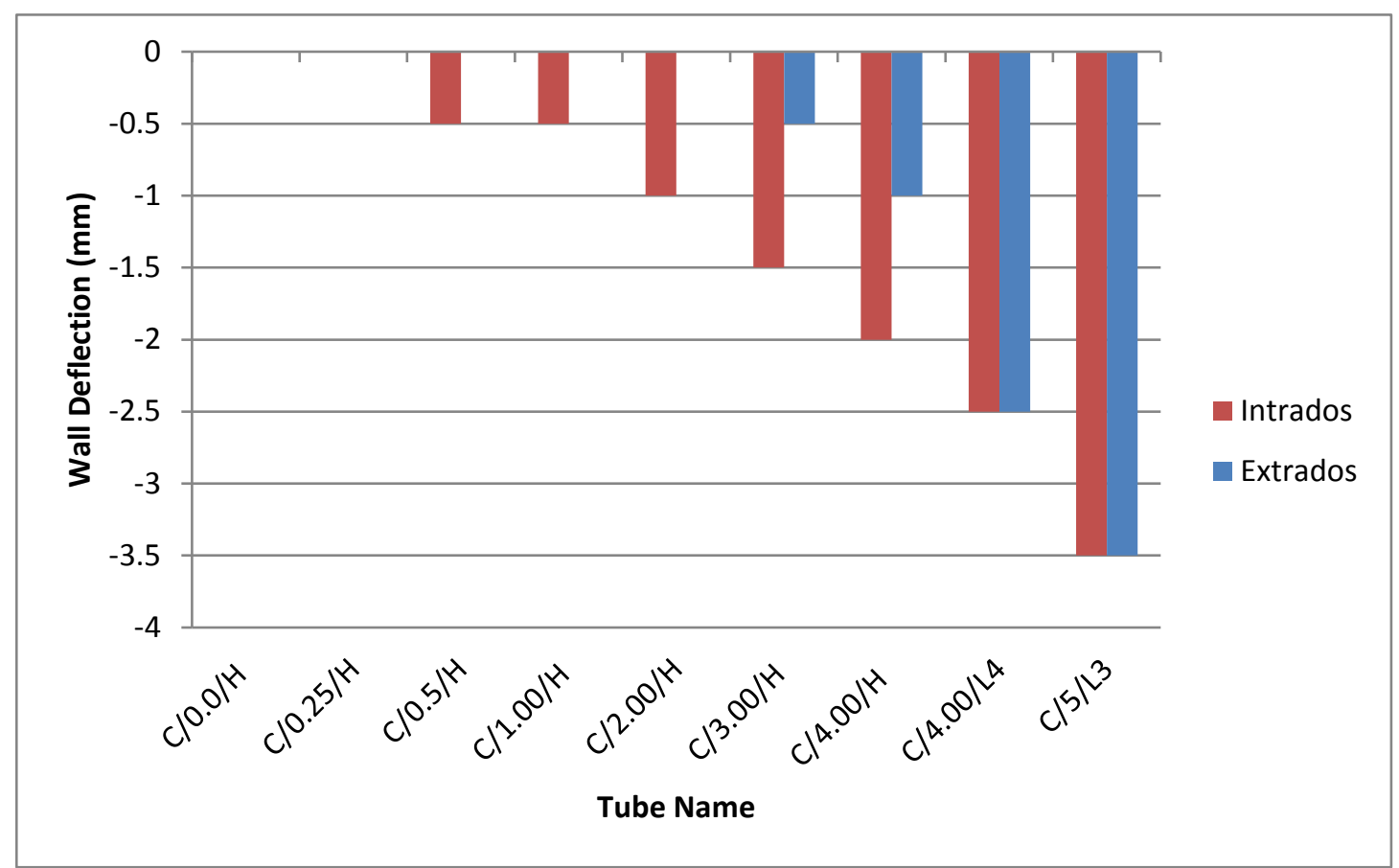

Figure 105-Cold-formed RHS Wall Deflection

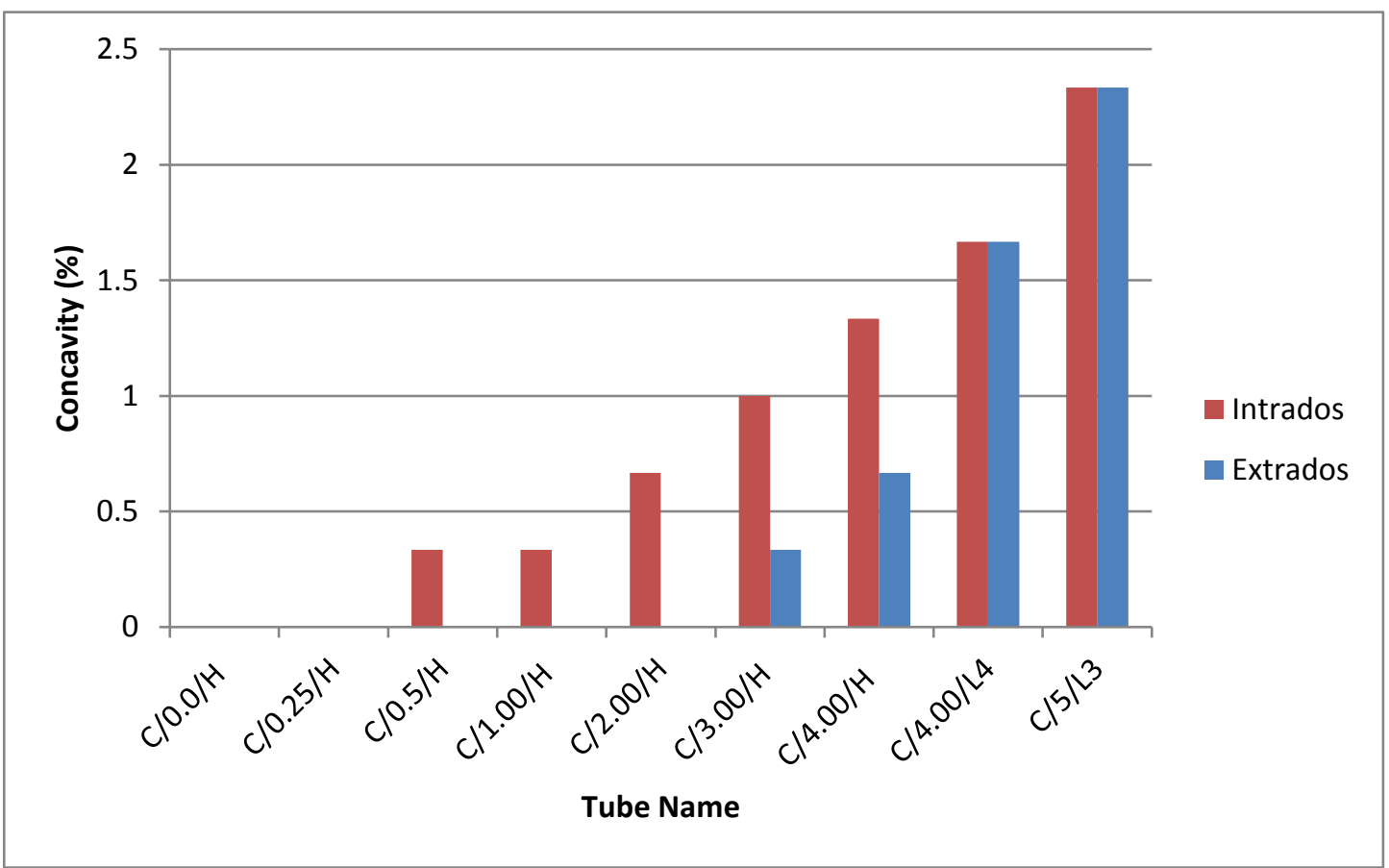

Figure 106-Cold-formed RHS Wall Concavity 
The Intrados experiences a more rapid increase in wall concavity at lower strains. This is likely caused by a combination of two mechanisms; firstly, the corners at the intrados are compressed into the forming rollers, adding more material to the intrados. The material at the intrados will also increase in thickness and width brought about by the conservation of volume as the material is compressed longitudinally. This lateral deformation is not proportional for plastically deformed material. These mechanisms cause the increase in width of the intrados wall. However, the section is constrained by the forming rollers; this produces a large compressive force across the intrados causing it to buckle.

The cause for the collapse of the extrados is less clear; the area is in tension and therefore was expected to experience a reduction in thickness and breadth. However, this was not observed in practice. Extension of a $750 \mathrm{~mm}$ section at the extrados is $37.5 \mathrm{~mm}$ when bent to $5 \%$ stain. Reducing the distance from the neutral axis reduces the extension required to achieve the same curvature. The section wall will extend by the minimum possible amount in order to reduce internal stresses. This will cause the unsupported wall to bow into the bore to reduce the extension made by the material. The hot-finished sections have a longer unsupported span at the extrados wall due to the tighter corner radii and therefore collapse earlier than the cold formed sections. With a $4 \mathrm{~mm}$ wall bow the strain is reduced by 0.0027 to 0.0473 . This equates to a reduction in extension of the centre wall of $2 \mathrm{~mm}$ over a $750 \mathrm{~mm}$ bend. The corner sections are too rigid to collapse in and therefore are pinned at the $5 \%$ strain position. A mandrel would cause all of the material (corners and flanges) to extend equally and would prevent this wall collapse from occurring.

Reducing the number of passes though the bending apparatus increases the wall concavity experienced at the intrados of both the hot-finished and cold-formed tubes. However, the extrados is affected differently. Reducing the pass number decreases the wall concavity of the extrados of the hot-finished section. The same is not true for the cold-formed section. 


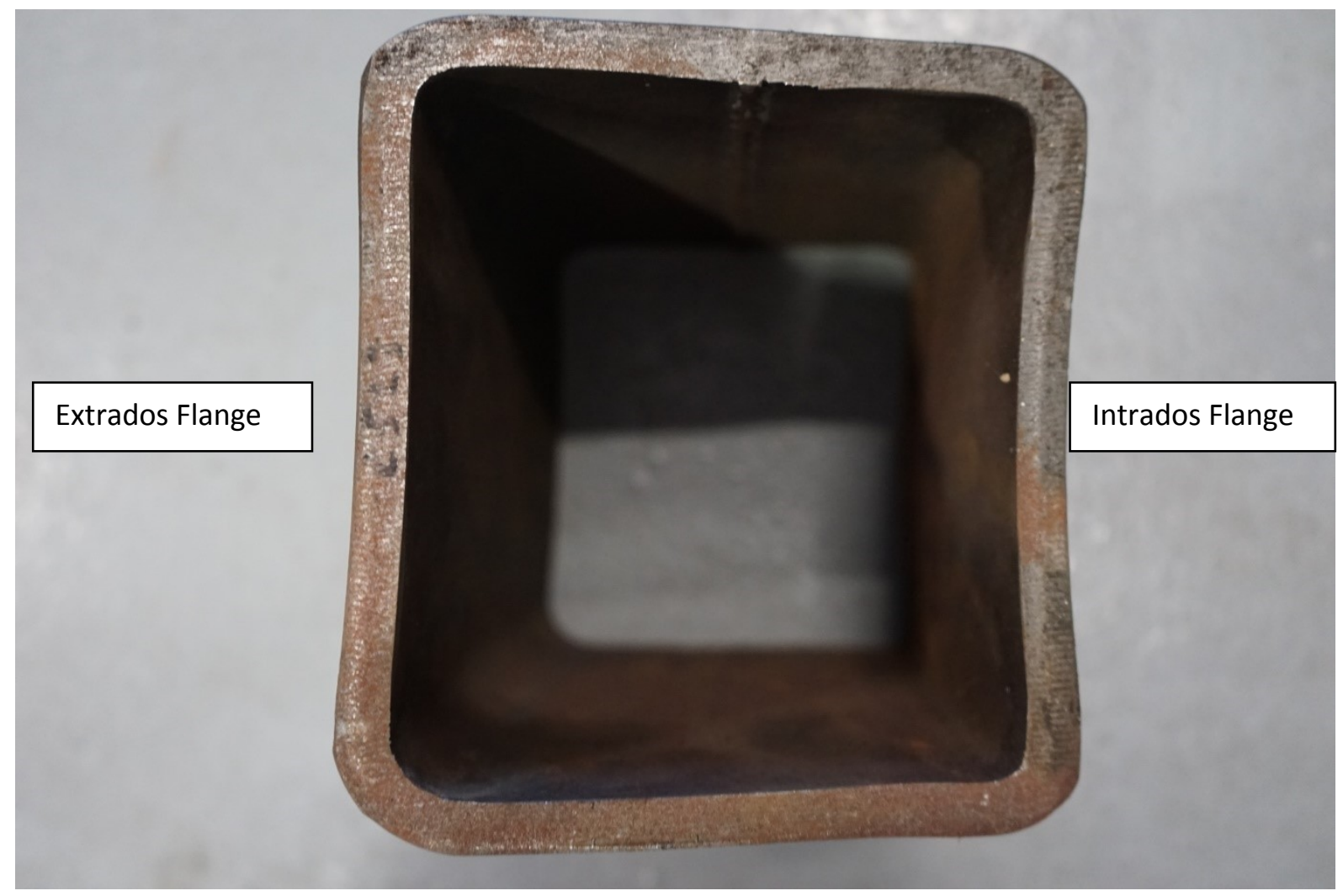

Figure 107-Intrados Concavity of a high strain cold-formed RHS

Figure 107 indicates the severity of the wall collapse of a cold-formed RHS bent to a maximum strain of $5 \%$. The intrados of the curve is on the right side of the section. The weld-line of the section was placed at the neutral axis of the curve. 


\subsubsection{Geometric Properties}

The measured dimensions of the sections were used to calculate the geometric properties. The geometric properties of a section are used to determine a section's suitability for use in a structure during the design process. The geometric properties of the sections have been calculated in order to assess the effect of the roller-bending process on the properties. The geometric property equations are stated in the respective standards for the hot-finished and cold-formed material[7], [9], [55], [56].

All of the geometric information is needed for correct engineering design of a structure. However, a number of effects of the bending process such as concavity of the walls of a RHS are not included in this list as they are assumed to lie within steel section specifications. Currently there is no construction standard for curved hollow sections; however, this is likely to change in the near future. All of the sections tested in this investigation have been compared against two standards; the production standard for straight sections (BS EN 10210:2006 and 10219:2006 for hot and cold formed sections respectively[7], [9], [55], [56]) and the construction standard for steel products (BS EN 1090:2009[4]). These comparisons will demonstrate the alterations in properties of the curved sections when compared to those of the straight sections and to assess how well they will perform in-situ.

The second moment of area is important for use in construction calculations as it is property of shape which aids the prediction of deflection, bending and stress under loading. It has been calculated in both the $\mathrm{X}-\mathrm{X}$ and $\mathrm{Y}-\mathrm{Y}$ directions using the mean wall thickness as well as the mean height and width dimensions. 


\begin{tabular}{|c|c|c|c|c|c|c|c|c|}
\hline $\begin{array}{l}\text { Specified } \\
\text { side } \\
\text { dimension, } \\
\text { D/B }\end{array}$ & $\begin{array}{l}\text { Specified } \\
\text { thickness, T }\end{array}$ & $\begin{array}{l}\text { Mass per } \\
\text { unit } \\
\text { length, } \mathrm{M}\end{array}$ & $\begin{array}{l}\text { Cross- } \\
\text { sectional } \\
\text { area, A }\end{array}$ & $\begin{array}{l}\text { Second } \\
\text { moment } \\
\text { of area, I }\end{array}$ & $\begin{array}{l}\text { Radius of } \\
\text { gyration, } \\
\text { i }\end{array}$ & $\begin{array}{l}\text { Elastic } \\
\text { section } \\
\text { modulus, } \\
\text { Wel }\end{array}$ & $\begin{array}{l}\text { Plastic } \\
\text { section } \\
\text { modulus, } \\
\text { Wpl }\end{array}$ & $\begin{array}{l}\text { Torsional } \\
\text { inertia } \\
\text { constant, It }\end{array}$ \\
\hline$(\mathrm{mm})$ & $(\mathrm{mm})$ & $(\mathrm{kg} / \mathrm{m})$ & $\left(\mathrm{cm}^{2}\right)$ & $\left(\mathrm{cm}^{4}\right)$ & $\mathrm{cm}$ & $\left(\mathrm{cm}^{3}\right)$ & $\left(\mathrm{cm}^{3}\right)$ & $\left(\mathrm{cm}^{4}\right)$ \\
\hline 150 & 5,0 & 22,6 & 28,7 & 1002 & 5,90 & 134 & 156 & 1550 \\
\hline 150 & 6,3 & 28,1 & 35,8 & 1223 & 5,85 & 163 & 192 & 1909 \\
\hline 150 & 8,0 & 35,1 & 44,8 & 1491 & 5,77 & 199 & 237 & 2351 \\
\hline 150 & 10,0 & 43,1 & 54,9 & 1773 & 5,68 & 236 & 286 & 2832 \\
\hline 150 & 12,5 & 52,7 & 67,1 & 2080 & 5,57 & 277 & 342 & 3375 \\
\hline 150 & 14,2 & 58,9 & 75,0 & 2262 & 5,49 & 302 & 377 & 3707 \\
\hline 150 & 16,0 & 65,2 & 83,0 & 2430 & 5,41 & 324 & 411 & 4026 \\
\hline
\end{tabular}

Table 17-Dimensions and sectional properties of a limited range of hot-finished RHS highlighting those bent during 


\begin{tabular}{|c|c|c|c|c|c|c|c|c|}
\hline $\begin{array}{l}\text { Specified } \\
\text { side } \\
\text { dimension, } \\
\text { D/B }\end{array}$ & $\begin{array}{l}\text { Specified } \\
\text { thickness, T }\end{array}$ & $\begin{array}{l}\text { Mass per } \\
\text { unit } \\
\text { length, } \mathrm{M}\end{array}$ & $\begin{array}{l}\text { Cross- } \\
\text { sectional } \\
\text { area, A }\end{array}$ & $\begin{array}{l}\text { Second } \\
\text { moment } \\
\text { of area, I }\end{array}$ & $\begin{array}{l}\text { Radius of } \\
\text { gyration, } \\
\text { i }\end{array}$ & $\begin{array}{l}\text { Elastic } \\
\text { section } \\
\text { modulus, } \\
\text { Wel }\end{array}$ & $\begin{array}{l}\text { Plastic } \\
\text { section } \\
\text { modulus, } \\
\text { Wpl }\end{array}$ & $\begin{array}{l}\text { Torsional } \\
\text { inertia } \\
\text { constant, It }\end{array}$ \\
\hline$(\mathrm{mm})$ & $(\mathrm{mm})$ & $(\mathrm{kg} / \mathrm{m})$ & $\left(\mathrm{cm}^{2}\right)$ & $\left(\mathrm{cm}^{4}\right)$ & $\mathrm{cm}$ & $\left(\mathrm{cm}^{3}\right)$ & $\left(\mathrm{cm}^{3}\right)$ & $\left(\mathrm{cm}^{4}\right)$ \\
\hline 150 & 4.0 & 18.0 & 22.9 & 808 & 5.93 & 108 & 125 & 1265 \\
\hline 150 & 5.0 & 22.3 & 28.4 & 982 & 5.89 & 131 & 153 & 1554 \\
\hline 150 & 6.0 & 26.4 & 33.6 & 1146 & 5.84 & 153 & 180 & 1833 \\
\hline 150 & 6.3 & 27.4 & 34.8 & 1174 & 5.8 & 156 & 185 & 1922 \\
\hline 150 & 8.0 & 33.9 & 43.2 & 1412 & 5.71 & 188 & 226 & 2364 \\
\hline 150 & 10.0 & 41.3 & 52.6 & 1653 & 5.61 & 220 & 269 & 2839 \\
\hline 150 & 12.0 & 47.1 & 60.1 & 1780 & 5.44 & 237 & 298 & 3231 \\
\hline 150 & 12.5 & 48.7 & 62.0 & 1817 & 5.41 & 242 & 306 & 3321 \\
\hline 150 & 16.0 & 58.7 & 74.8 & 2009 & 5.18 & 268 & 351 & 3830 \\
\hline
\end{tabular}

Table 18-Dimensions and sectional properties of a limited range of cold-formed RHS highlighting those bent during 


\subsubsection{Second Moment of Area, I}

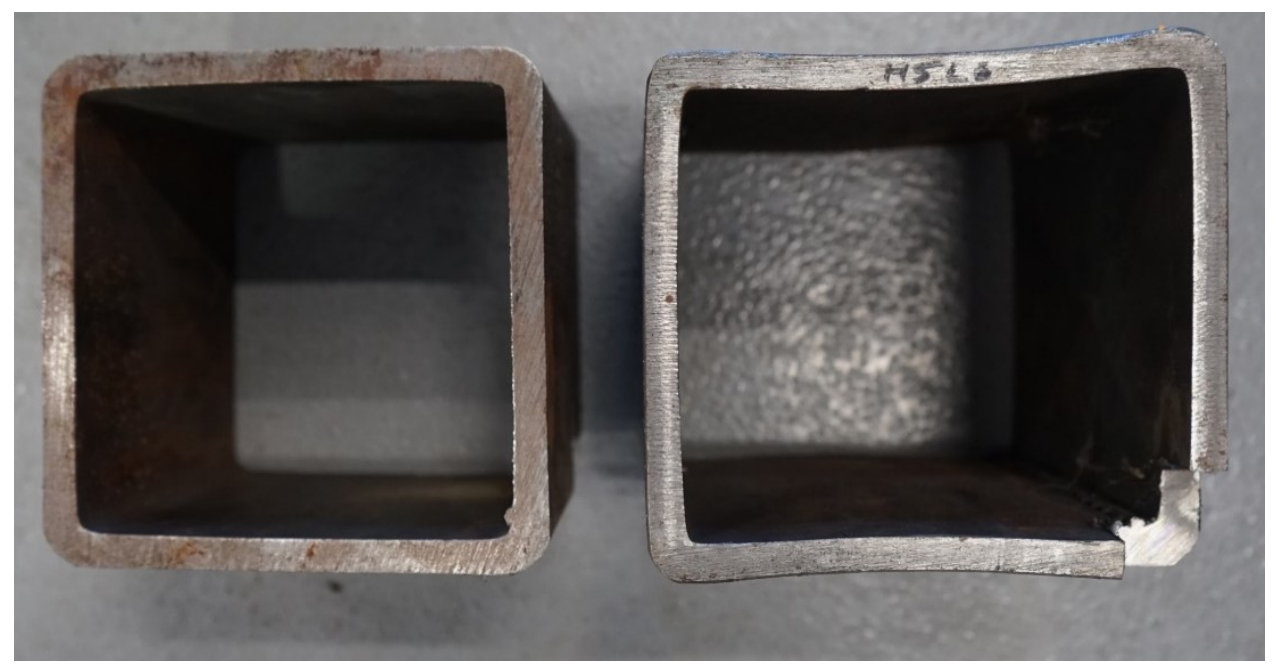

Figure 108-Geometry alteration in hot-finished RHS due to deformation

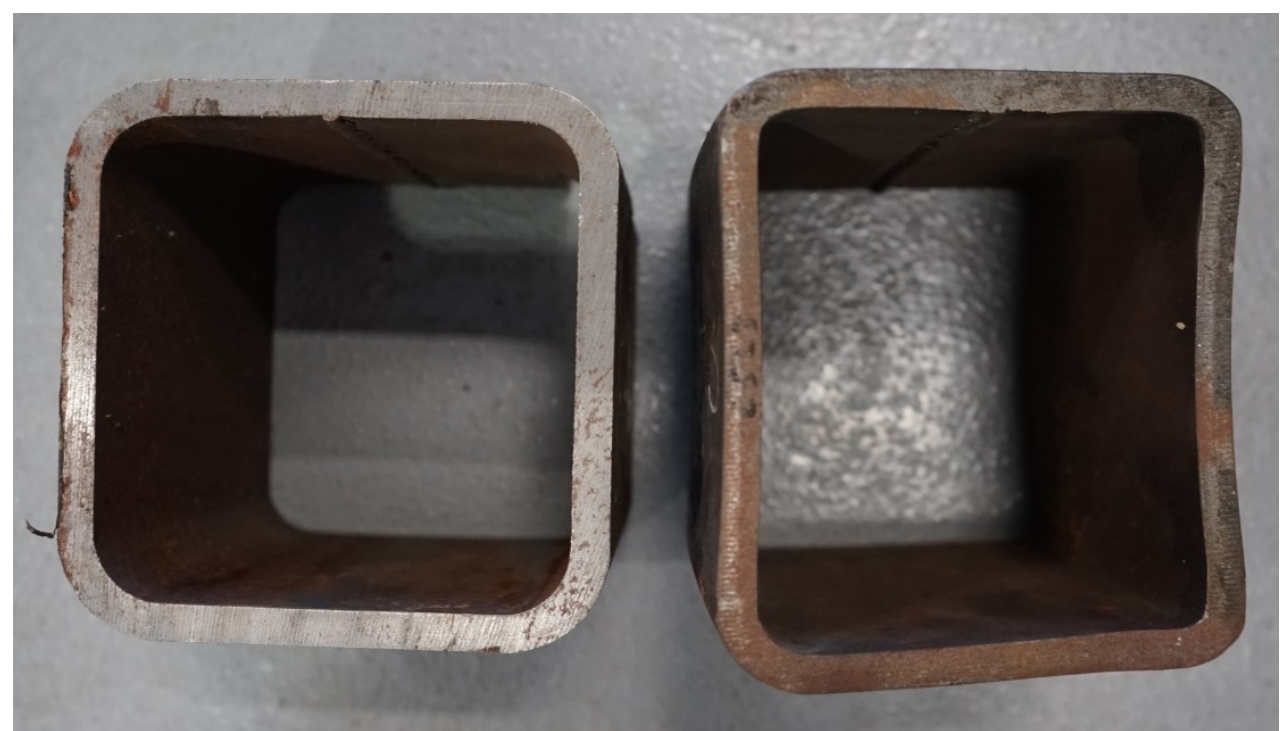

Figure 109-Geometry alteration in cold-formed RHS due to deformation 


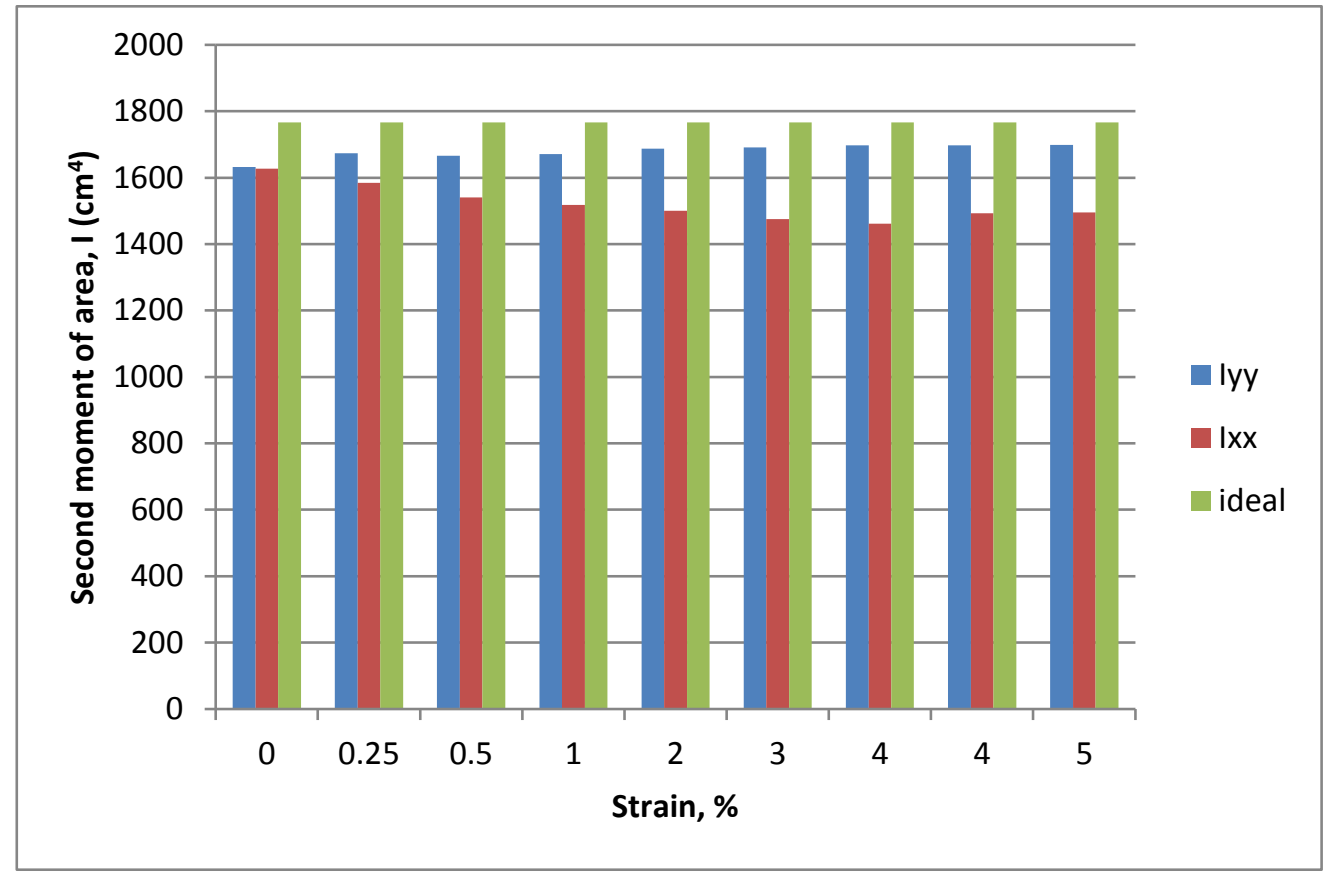

Figure 110-Graph plotting the second moment of area, I as strain is increased for hot-finished RHS

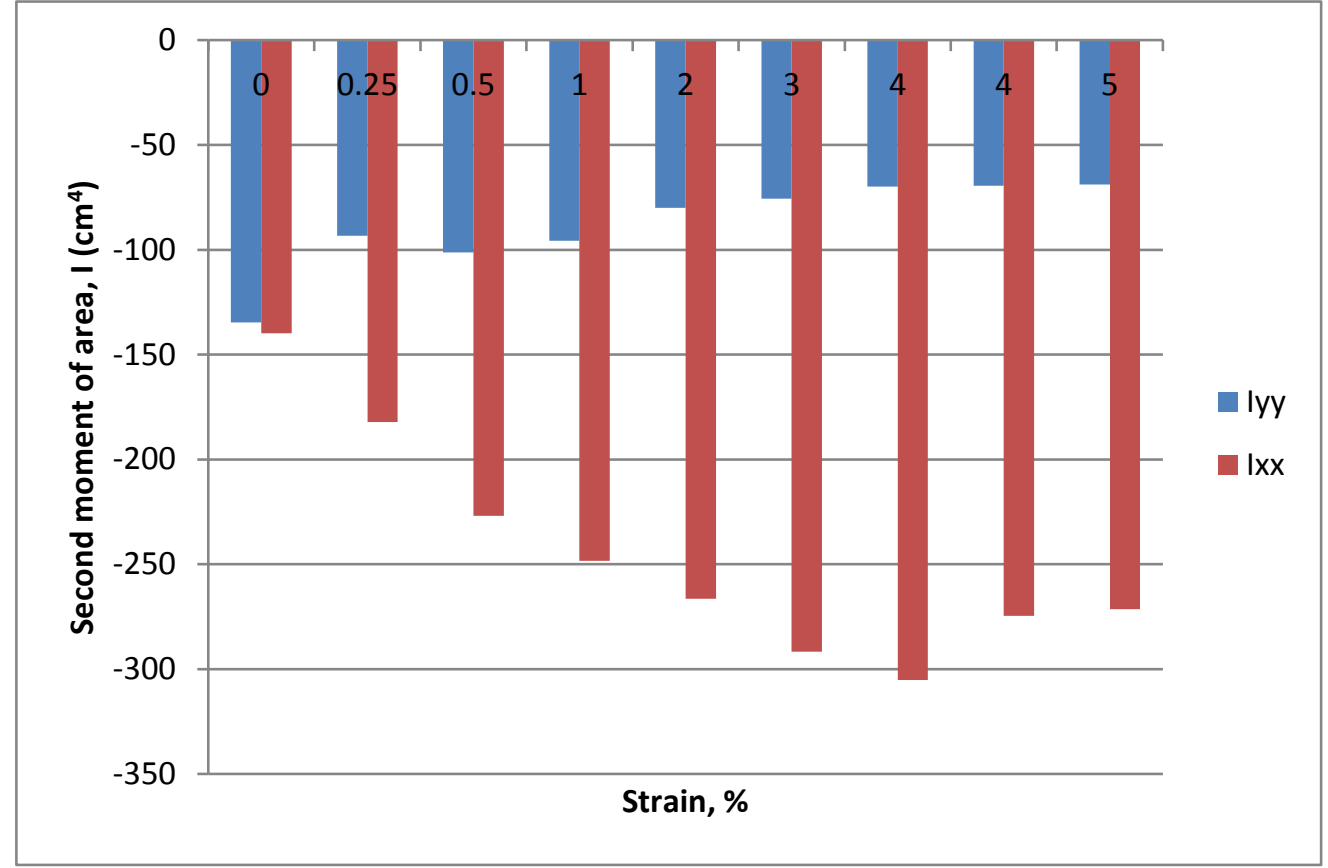

Figure 111-Graph plotting deviation in the second moment of area, I from that quoted in the specification as strain is increased for hot-finished RHS 
The second moment of area of the as delivered product should be approximately $1773 \mathrm{~cm}^{4}$ as stated in BS EN 10210-2:2006[7], [9]. The received material had an unbent second moment of area of approximately $1653 \mathrm{~cm}^{4}$ which is significantly lower than the ideal value. This reduction is due to the reduced wall thickness $(<10 \mathrm{~mm})$. Initially, the second moment of area in both the $\mathrm{XX}$ and $\mathrm{Y}-\mathrm{Y}$ directions is approximately equal as the section has not yet been deformed. This would mean that deformation in either the $X-X$ or $Y-Y$ direction would require an approximately equal force. As the wall thickness and outside dimensions change, so does the second moment of area. The resistance of a section in a given direction is altered not only by the cold work but the change in geometry. For example (discounting mechanical properties of the steel) as the $I_{y-y}$ increases so does the resistance to bending in that plane. Conversely, a fall in the $I_{x-x}$ would lead to reduced resistance within the section along the $X-X$ plane. This means that the sections resistance to being bent back has been reduced so discounting the mechanical properties, the resistance to bending in the $x x$ direction has been reduced. 


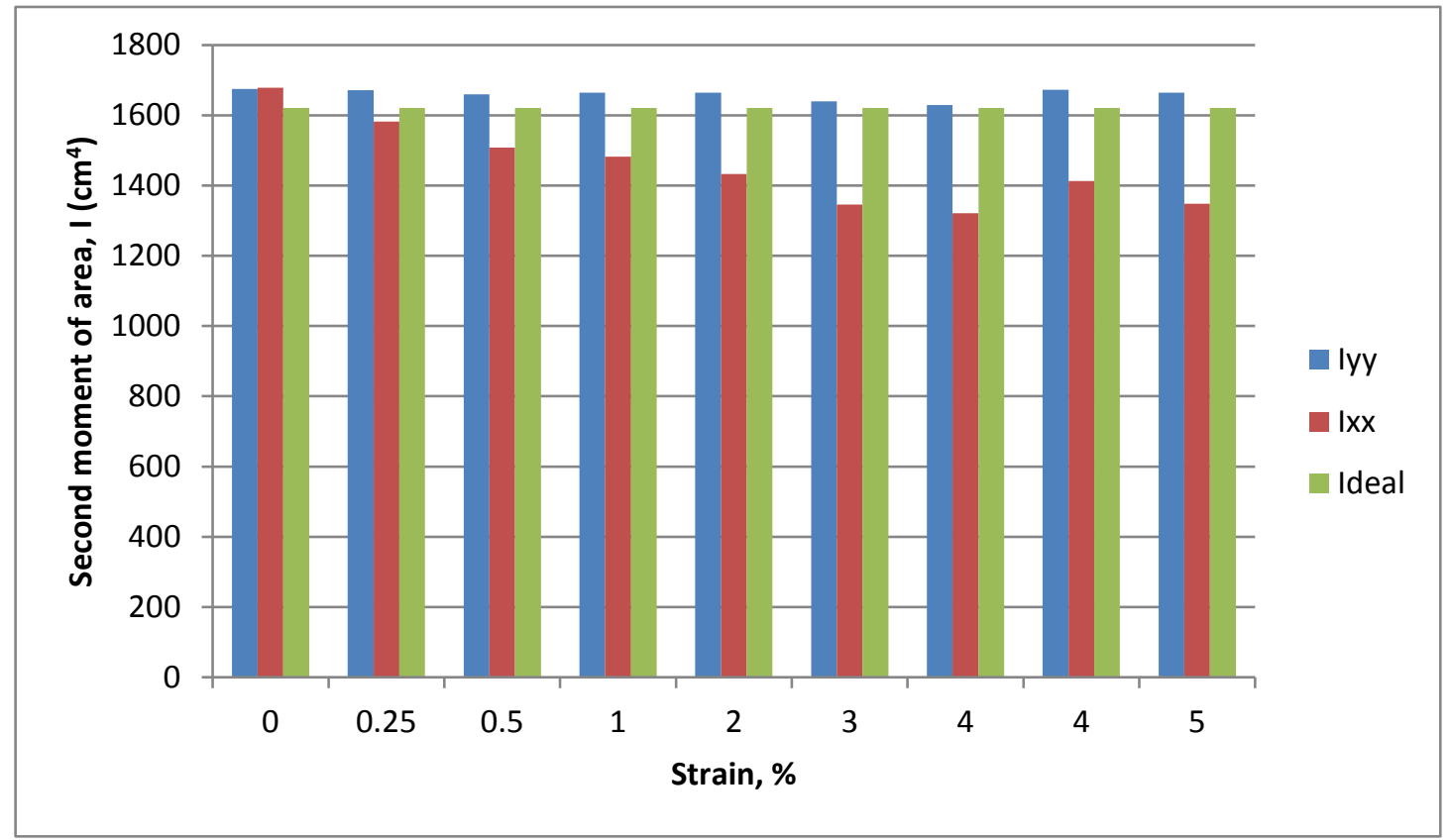

Figure 112-Graph plotting change in second moment of area, I as strain is increased for cold-formed RHS

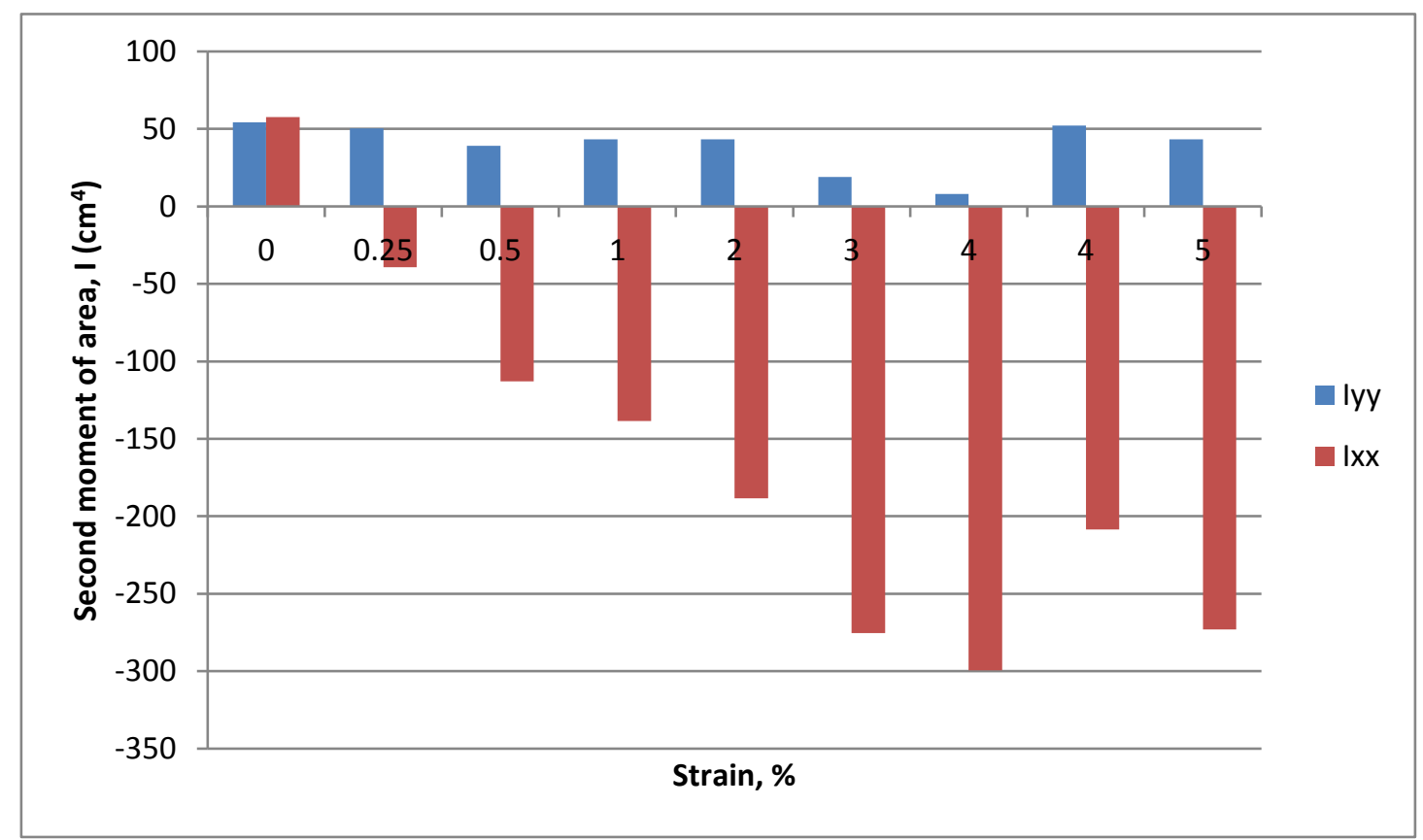

Figure 113-Graph plotting deviation in the second moment of area, I from that quoted in the specification as strain is increased for cold-formed RHS 
The second moment of area of the as-delivered product should be $1653 \mathrm{~cm}^{4}$ as stated in BS EN 10219-2:2006[55], [56]. The received material had an unbent second moment of area of approximately $1675 \mathrm{~cm}^{4}$. The cold-formed section has a higher unbent second moment of area than the hot-finished equivalent which is due to the larger external dimensions. However the coldformed RHS sees a large initial drop in I in the X-X direction of approximately $100 \mathrm{~cm}^{4}$. It also suffers a larger drop in $I_{x x}$ at higher strain; this is likely due to the large initial corner radii. However, due to the initially low second moment of area of the hot-finished RHS both have a second moment of area $300 \mathrm{~cm}^{4}$ less than the ideal by $4 \%$ strain. It can also be seen that a lower pass number reduces this drop in $I_{x x}$.

The cold-formed RHS has a total reduction in I of $350 \mathrm{~cm}^{4}$ whereas the hot-finished experiences a reduction of only $300 \mathrm{~cm}^{4}$ by $4 \%$ strain. Reducing the number of passes has a larger effect on the $I_{x x}$ of the cold-formed RHS. The second moment of area of the low pass number RHS has fallen to only $200 \mathrm{~cm}^{4}$ below the ideal compared. The high strain RHS experiences a further $100 \mathrm{~cm}^{4}$ reduction. The effect of reducing the pass number on the hot-finished section is much less significant with a reduction of approximately $25 \mathrm{~cm}^{4}$ less. 


\subsubsection{Radius of Gyration}

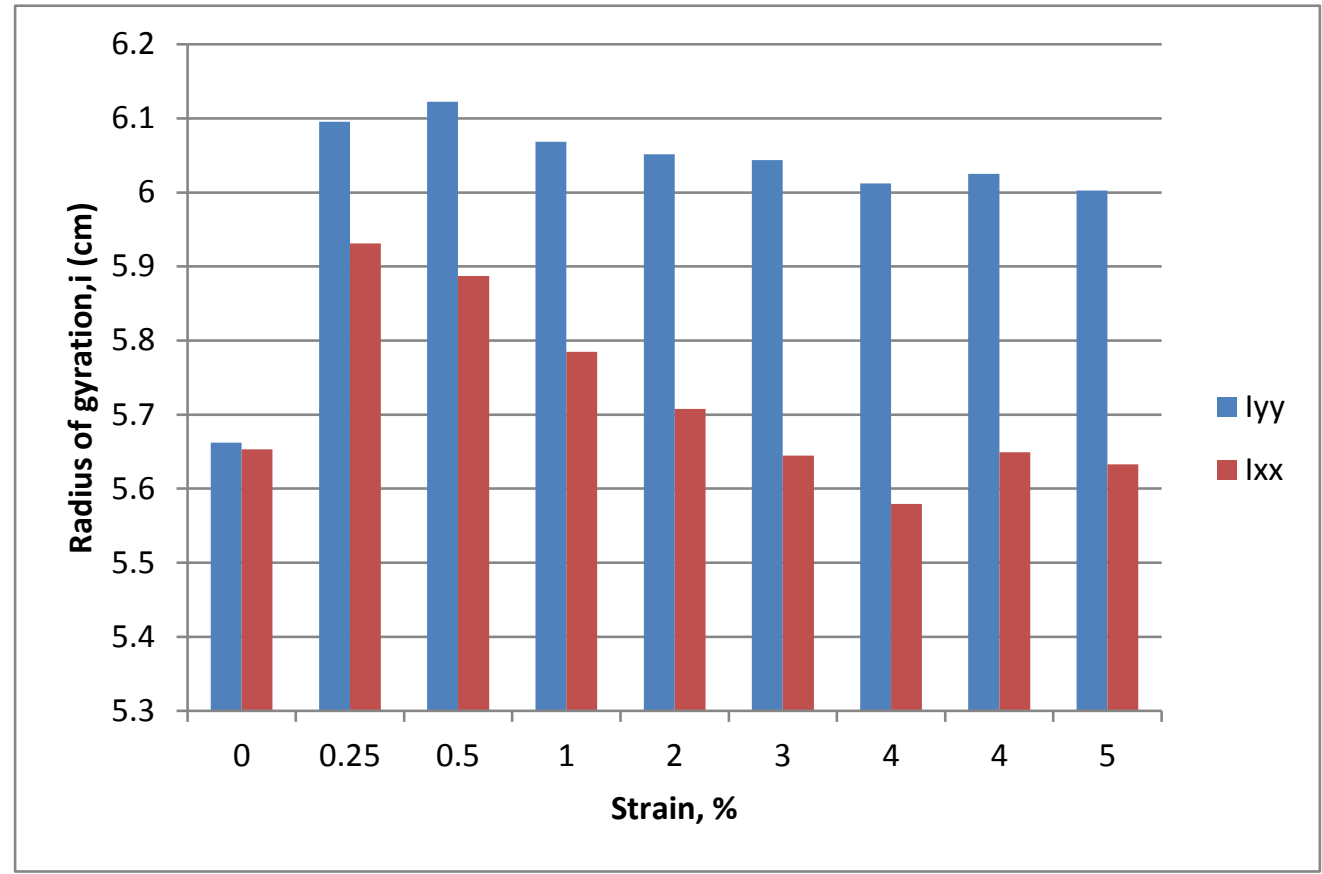

Figure 114-Graph plotting change in radius of gyration, $i$ as strain is increased for hot-finished RHS

The unbent radius of gyration stated for a hot-finished RHS of 150x150x10 in EN10210-2:2006 is $5.68 \mathrm{~cm}[55],[56]$. The recorded value is approximately equal to the specification. It can be seen that there is a sharp initial increase in the radius of gyration in both directions before a steady decline as strain rate is increased. The $\mathrm{X}-\mathrm{X}$ direction experienced a more rapid decline than the $\mathrm{Y}-\mathrm{Y}$. This is due to the narrowing of the cross section. The increased radius of gyration would increase the sections capacity to resist buckling under axial compression, however, the sections curved shape would counteract this, making the section more likely to buckle under axial loading. The smallest value in any direction is used for structural calculations as it is the most likely to buckle 


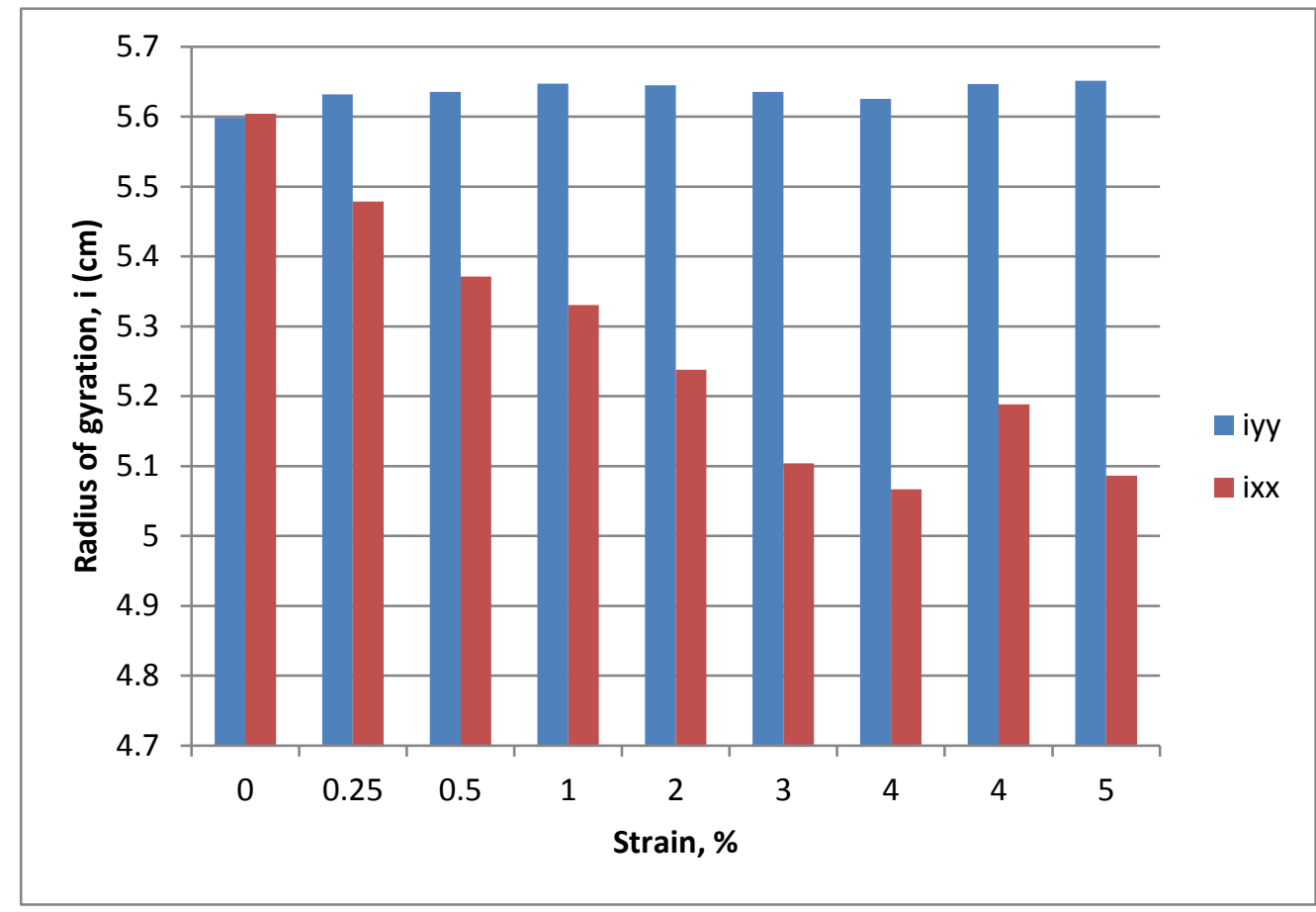

Figure 115-Graph plotting change in radius of gyration, $i$ as strain is increased for cold-formed RHS

The unbent radius of gyration stated in EN10219-2:2006 is 5.61 cm[7], [9]. It can be seen from Figure 115 that the $\mathrm{Y}-\mathrm{Y}$ direction experiences a small increase in radius of gyration and the intrados experiences a reduction in radius of gyration. The reduction in the radius of gyration equates to a reduction in the sections capacity to resist buckling under axial compression. This property of the cross section, coupled with the curved shape of the section will lead to a significantly reduces capacity for buckling resistance. 


\subsubsection{Elastic section Modulus}

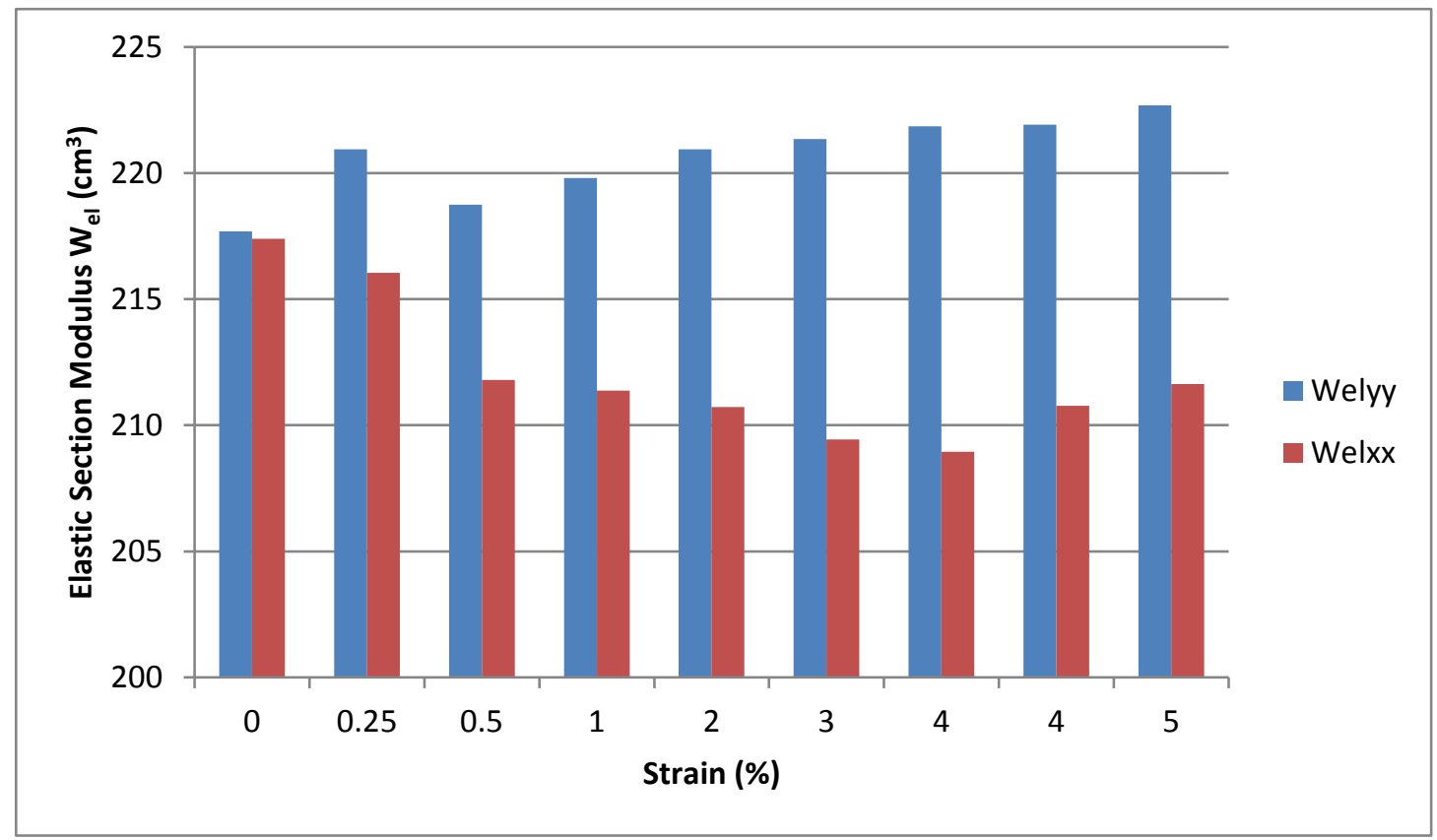

Figure 116-Graph plotting change in elastic section modulus, $W_{e l}$ as strain is increased for hot-finished RHS

The elastic section modulus for a hot-finished RHS of $150 \times 150 \times 10 \mathrm{~mm}$ in EN10210-2:2006 is $236 \mathrm{~cm}^{3}[55],[56]$. The recorded elastic section modulus is less than the quoted figure at $218 \mathrm{~cm}^{3}$. This is likely due to the corner radii of the product and the reduced wall thickness. Figure 116 plots the change in elastic section modulus as strain rate is increased. It can be seen that the modulus in the $\mathrm{Y}-\mathrm{Y}$ direction is increased and the modulus in the $\mathrm{X}-\mathrm{X}$ direction is decreased. A reduction in elastic section modulus will equate to a reduced section yielding moment. The lower the elastic section modulus, the lower the required bending moment required to yield the section. 


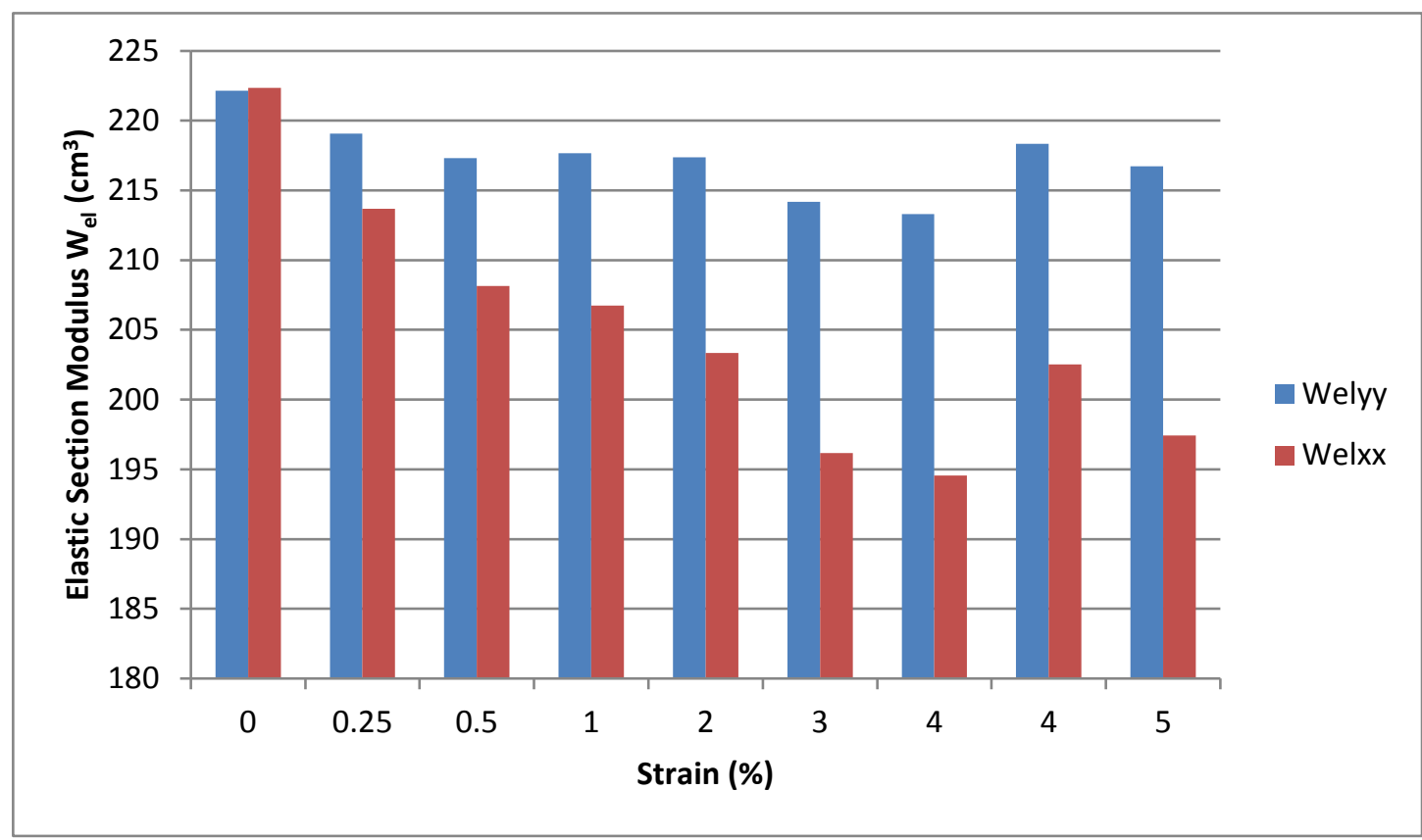

Figure 117-Graph plotting change in elastic section modulus, $W_{e l}$ as strain is increased for cold-formed RHS

The elastic section modulus for an RHS of $150 \times 150 \times 10 \mathrm{~mm}$ in EN10219-2:2006 is $220 \mathrm{~cm}^{3}$ [7], [9]. The recorded elastic section modulus is higher than the quoted figure at approximately $223 \mathrm{~cm}^{3}$. Figure 117 plots the change in elastic section modulus as strain rate is increased. It can be seen that the modulus in both directions is decreased. As with the hot-finished section, this will equate to a reduced section yielding moment in both the $\mathrm{X}-\mathrm{X}$ and $\mathrm{Y}-\mathrm{Y}$ directions. The lower the elastic section modulus, the lower the required bending moment required to yield the section. 


\subsubsection{Plastic section modulus}

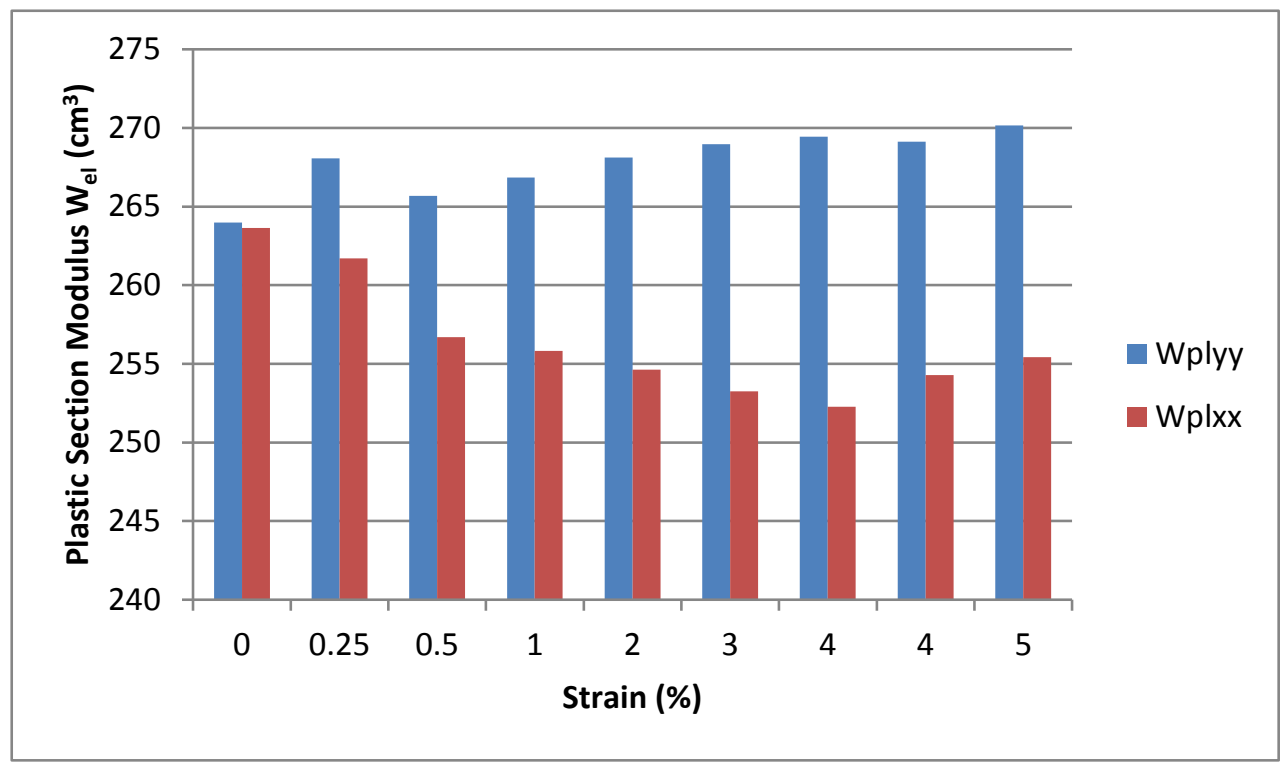

Figure 118-Graph plotting change in plastic section modulus, $W_{p l}$ as strain is increased for hot-finished RHS

The plastic section modulus for a hot-finished RHS of $150 \times 150 \times 10 \mathrm{~mm}$ in EN10210-2:2006 is $286 \mathrm{~cm}^{3}[55],[56]$. The recorded unbent plastic section modulus is less than the quoted figure at $264 \mathrm{~cm}^{3}$. As with the elastic section modulus, the plastic section modulus increases in the $Y-Y$ direction and decreases in the $\mathrm{X}-\mathrm{X}$ direction.

Many designers will not use the plastic section modulus in their design calculations as plastic deformation in any member of a superstructure is usually avoided. 


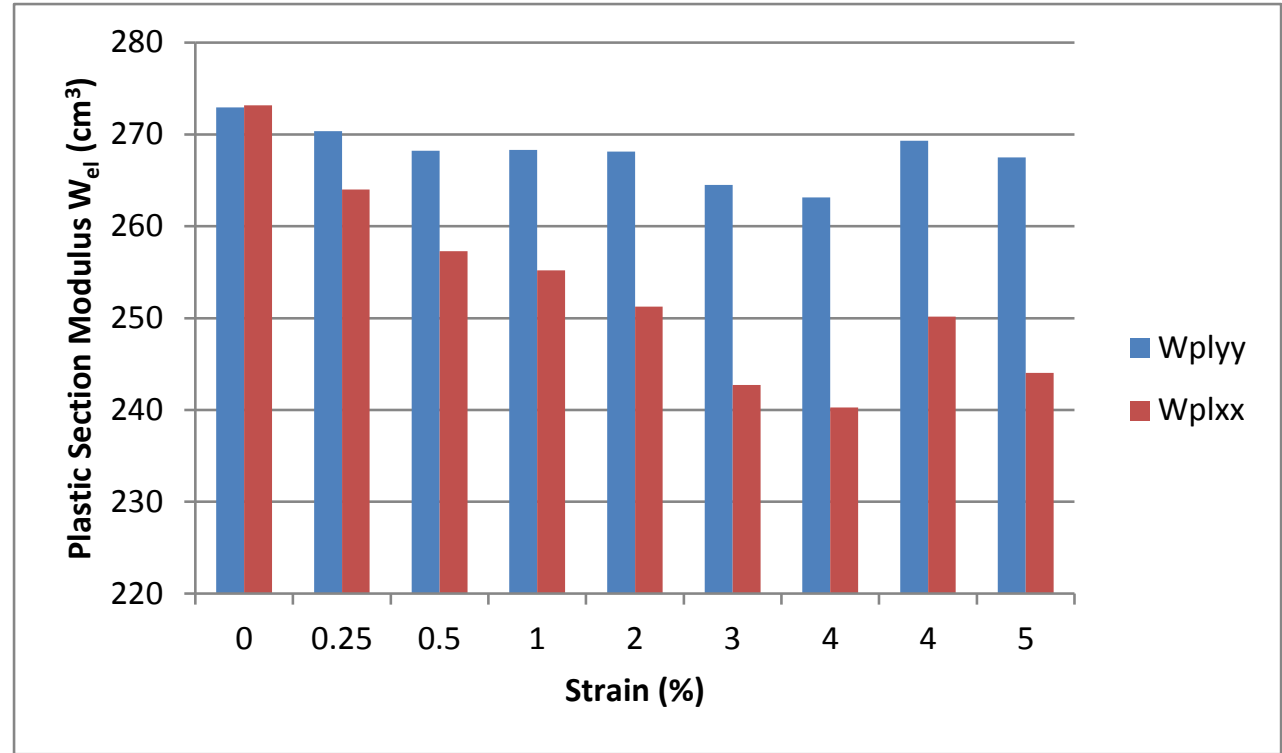

Figure 119-Graph plotting change in plastic section modulus, $W_{p l}$ as strain is increased for cold-formed RHS

The plastic section modulus for an RHS of $150 \times 150 \times 10 \mathrm{~mm}$ in EN10219-2:2006 is $269 \mathrm{~cm}^{3}$ [7], [9]. The recorded unbent plastic section modulus is higher than the quoted figure at $274 \mathrm{~cm}^{3}$. As with the elastic section modulus, the plastic section modulus decreases in both the $\mathrm{Y}-\mathrm{Y}$ and $\mathrm{X}-\mathrm{X}$ directions. Many designers will not use the plastic section modulus in their design calculations as plastic deformation in any member of a superstructure is usually avoided. 


\subsubsection{Torsional Inertia Constant}

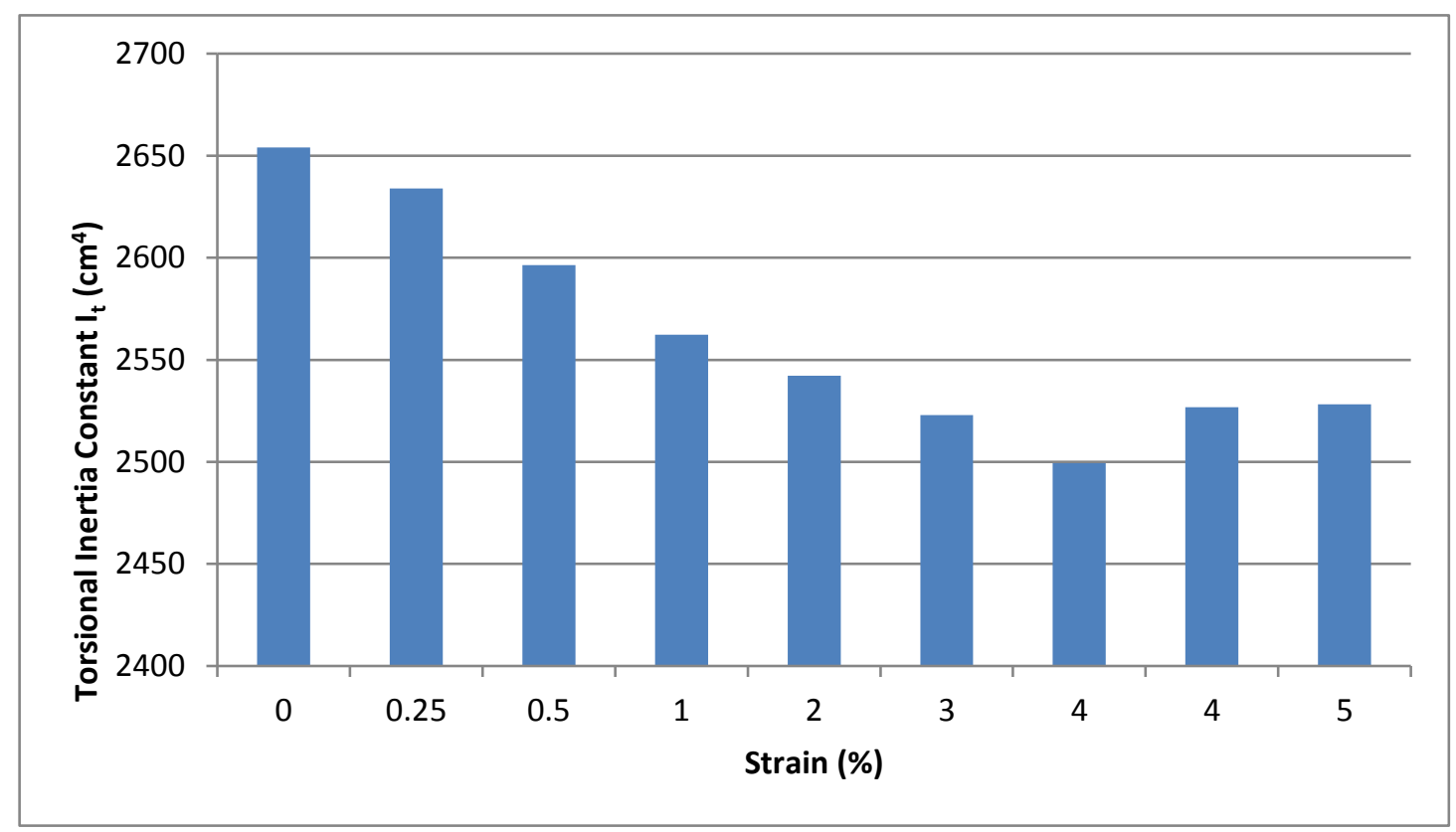

Figure 120-Graph plotting change in torsional inertia constant, $I_{t}$ as strain is increased for hot-finished RHS

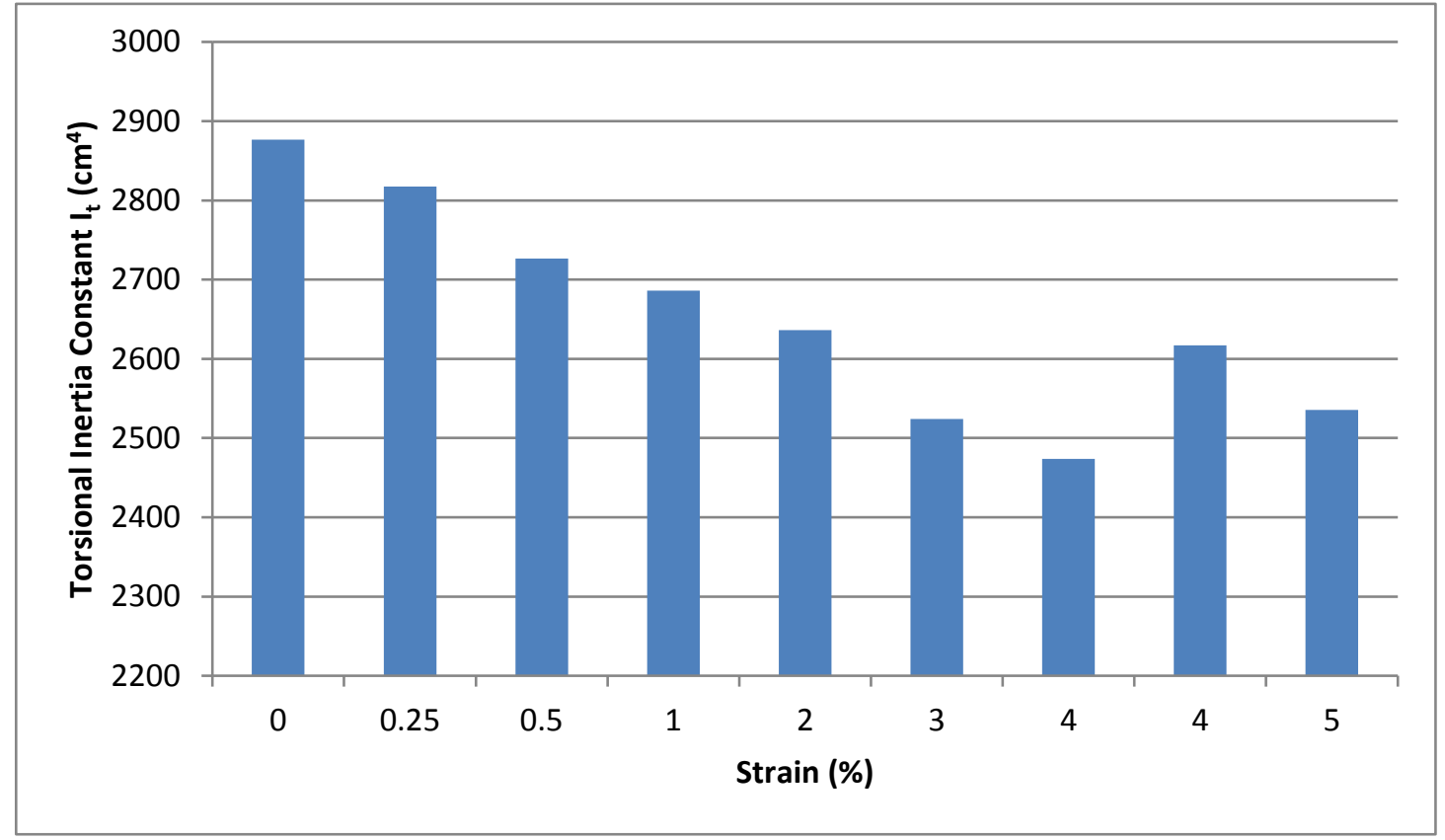

Figure 121-Graph plotting change in torsional inertia constant $I_{t}$ as strain is increased for cold-formed RHS 


\subsubsection{Torsional Modulus Constant}

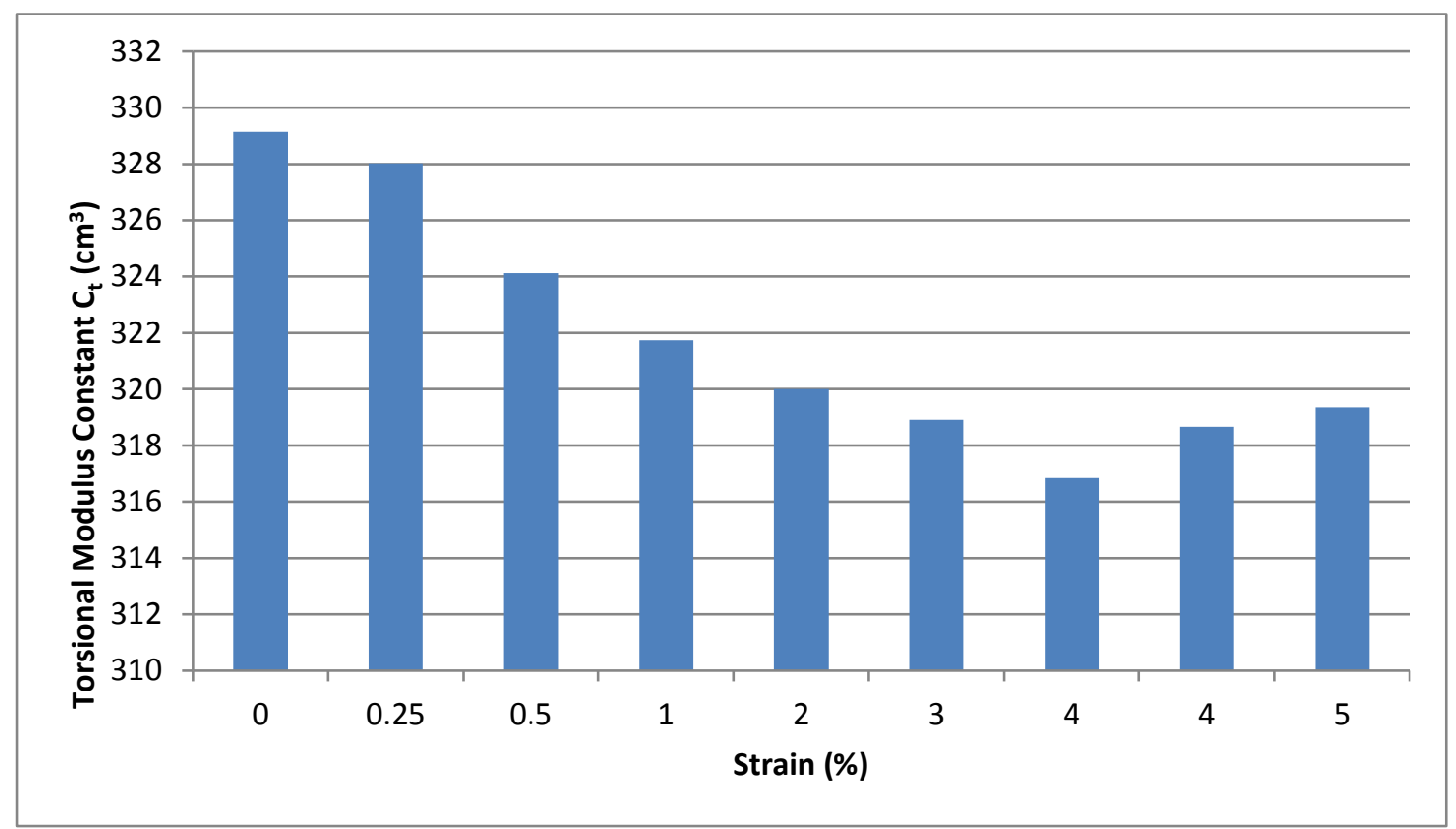

Figure 122-Graph plotting change in torsional modulus constant, $C_{t}$ as strain is increased for hot-finished RHS

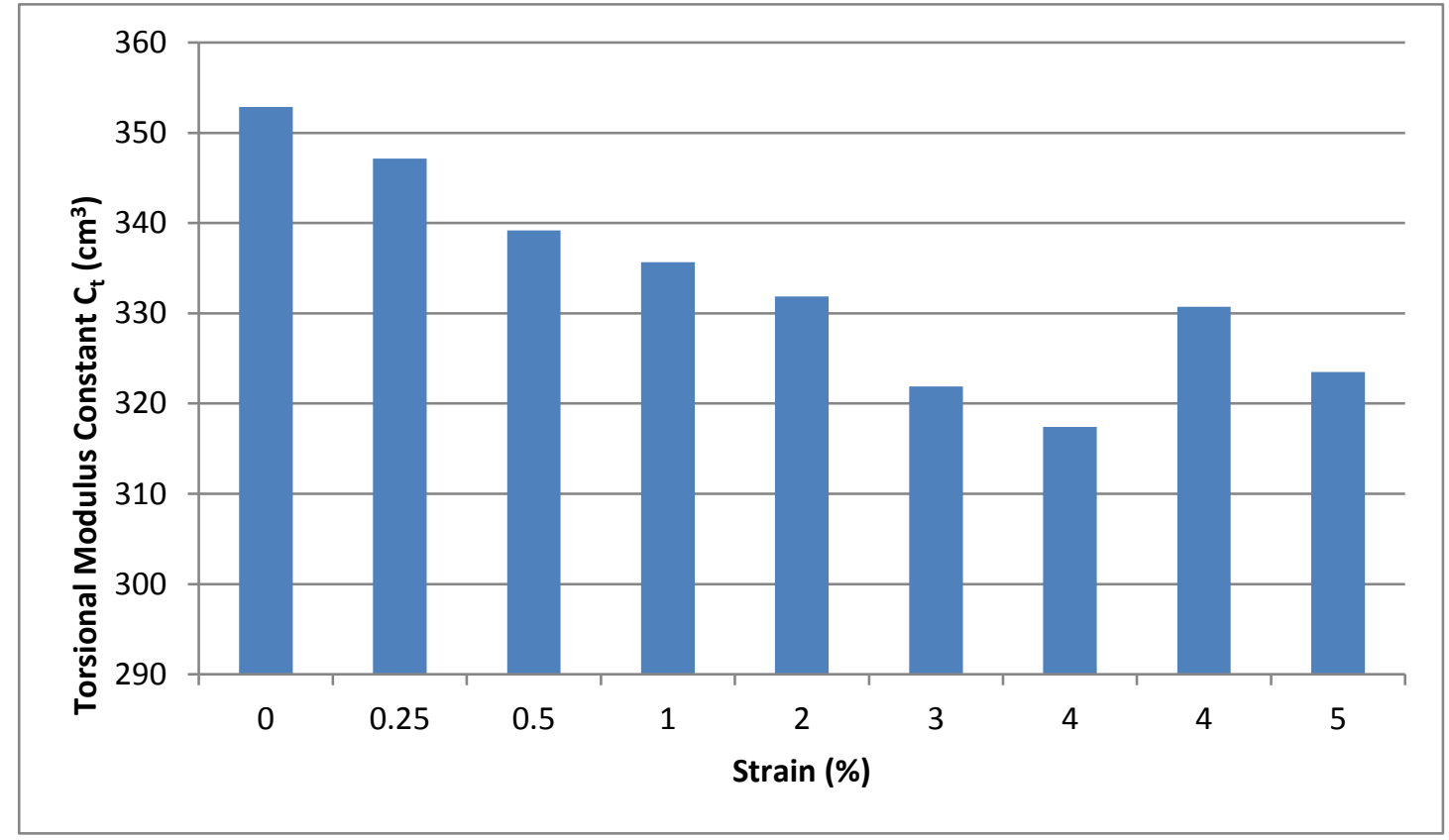

Figure 123-Graph plotting change in torsional modulus constant, $C_{t}$ as strain is increased for cold-formed RHS

The torsional inertia constant for a hot-finished RHS of $150 \times 150 \times 10 \mathrm{~mm}$ in EN10210-2:2006 is $2832 \mathrm{~cm}^{4}$. The recorded unbent torsional inertia constant is less than the quoted figure at $2650 \mathrm{~cm}^{4}$. 
The torsional modulus constant for a hot-finished RHS of $150 \times 150 \times 10 \mathrm{~mm}$ in EN10210-2:2006 is $344 \mathrm{~cm}^{3}$. The recorded unbent torsional modulus constant is less than the quoted figure at $329 \mathrm{~cm}^{3} \cdot[55],[56]$

The torsional inertia constant for a cold-formed RHS of $150 \times 150 \times 10 \mathrm{~mm}$ in EN10219-2:2006 is $2839 \mathrm{~cm}^{4}$. The recorded unbent torsional inertia constant is greater than the quoted figure at approximately $2900 \mathrm{~cm}^{4}$.

The torsional modulus constant for a cold-formed RHS of $150 \times 150 \times 10 \mathrm{~mm}$ in EN10219-2:2006 is $341 \mathrm{~cm}^{3}$. The recorded unbent torsional modulus constant is greater than the quoted figure at approximately $353 \mathrm{~cm}^{3} .[7]$, [9]

It can be seen in Figure 120, Figure 121, Figure 122 and Figure 123 that both the torsional Inertia constant and torsional modulus constant decrease as strain rate is increased for both hot-finished and cold-formed tubes. A reduction in these properties equates to a reduction in the cross-sections capacity to resist torsion. The cold-formed section has a higher unbent resistance to torsion. However, after bending to $4 \%$ strain, both have torsional inertia constants of approximately 2500 $\mathrm{cm}^{4}$. This reduction is caused by the change in height, breadth and the wall thickness of the section. The above calculations are based on the cross section. However, the longitudinal shape of the tube will also affect the torsion of the section.

It can be seen that reducing the number of passes through the bending apparatus reduces the effect on the torsional resistance. 


\section{Discussion}

\subsection{Hot-finished RHS}

The Hot-finished RHS has an E22 grade chemistry which has a relatively low carbon equivalent value. This low CEV means that it has a minimal hardenability. The normalised production method and its lean chemistry mean that the hot-finished RHS has a fine; consistent microstructure with a fine dispersion of precipitates, no grain elongation or residual stresses. The normalising process eliminates any weld-line effects and homogenises the section grain structure. This is evident from the mechanical properties of the section before bending has taken place. The section has uniform hardness, tensile strength and impact resistance. The production of the corners does not introduce any stress into the section as the corners are produced above the transformation temperature. The section met all of the requirements of the BS EN 10210 in terms of physical dimensional tolerances before bending. Very little cold work is required in order to increase the hardness of the intrados corners Bent sections are not required to conform to the same rigorous standards as straight sections; however, all of the sections in this report have been compared against the straight length standards to illustrate the effect of the bending process. The hot-finished RHS would normally conform to the BS EN 10210.[55], [56]

The hot-finished RHS only showed signs of visible cold work at the corners at maximum strain. This can be seen in Figure 36. The average cross-sectional hardness of all areas of the tube increased with a maximum occurring at the extrados corner. The mean hardness of the outer corner increased by $30 \mathrm{HV}$. However, the neutral axis experienced a minor reduction.

The sections mostly fail to meet the dimensional requirements of BS EN 10210[55], [56]. The wall thickness however is the exception. The wall thickness of the section remains within specification even at the most extreme strains. The reason for this is that it is easier for the wall to bow when under compression than to increase in thickness. The other geometric requirements however do fail to meets some of the requirements of the standard. For example, the external dimensions fall out of specification at the first strain increment, $0.25 \%$. The breadth to depth ratio is approximately $9 \%$ by $4 \%$ strain. The collapse of the wall caused by the compression of the tube was evident, surpassing the failure threshold at $3 \%$ strain. The maximum concavity was experienced at $4 \%$ strain with a value of $2.25 \%$. All of these dimensional changes lead to a large increase in second moment of area in the $\mathrm{Y}-\mathrm{Y}$ direction. This can be seen in Figure 110.The value increases from approximately $1600 \mathrm{~cm}^{4}$ to $1700 \mathrm{~cm}^{4}$. However, this increase in second moment of area will improve the resistance to bending in the $Y-Y$ direction but any decrease I would mean a reduced resistance to bending in that direction. Therefore, the reduction in I X-X means that the section has a reduced capacity to 
resist bending in the $\mathrm{X}-\mathrm{X}$ direction. The second moment of area is an important value used in steel design calculations, any change brought about during the bending process must be calculated in order for accurate design calculations to be achieved. The decrease in second moment of area in the $X-X$ direction, coupled with the Bauschinger effect will result is a lower required stress to bend the section back in the opposite direction. If the section had been reformed using induction bending the internal stresses within a hot-finished member would be minimised due to the recrystallization. 


\subsection{Cold formed RHS}

The cold formed RHS is a TATA product produced in the Netherlands and has a relatively high carbon equivalent value. This high CEV means that the section is harder and is susceptible to hardening. The cold formed production method yields a hard microstructure with high internal stresses and elevated dislocation density. The weld-line has not been heat treated so a large HAZ can be observed. In contrast to the bulk material, a large quantity of acicular ferrite can be seen along the weld-line which is a sign of the rapid cooling during the forming process. This HAZ has a significantly greater hardness than the bulk material due to microstructure brought about by the rapid cooling rate and the chemistry of the steel. Due to the cold formation of the corners during production, they have a significantly greater hardness than the bulk material. Greater bending forces are required to deform the more rigid microstructure. A loud, high pitched sound was produced during bending of the cold-formed RHS which was not experienced during bending of the hot-finished products. This was brought about as the rollers slipped on the hard surface.

Bent sections are not required to conform to the same rigorous standards as straight sections; however, all of the sections in this report have been compared against the straight length standards to illustrate the effect of the bending process. The cold-formed RHS would normally conform to the BS EN 10219[7], [9].

As with the hot-finished RHS, only the corners showed visible signs of cold work, even at maximum strain. There was no significant change is surface hardness as the results are too variable. The average cross-sectional hardness of all areas of the tube increased with the exception of the neutral axis. The most notable increase was at the extrados corner which experienced an increase of $16 \mathrm{HV}$.

As with the hot-finished section, the sections failed to meet all of the dimensional requirements evaluated except wall thickness change. The wall thickness of the section remains within specification even at the most extreme strains. The other geometric requirements however do fail to meets some of the requirements. For example, the external dimensions fall out of specification at the first strain increment, $0.25 \%$ strain. The breadth to depth ratio is approximately $12 \%$ by $5 \%$ strain. The concavity of the walls was clearly evident, surpassing the failure threshold at $3 \%$ strain with a maximum at $5 \%$ strain of $2.25 \%$ concavity. The second moment of area of the cold-formed sections is therefore affected by this change in the depth to breadth ratio. Both planes experience an initial reduction in second moment of area. This is unlike the hot-finished section which experienced an increase in the $Y-Y$ direction and a decrease in the $X-X$ direction. This can be seen in Figure 112 . The $I_{Y-Y}$ value goes from the approximately $1675 \mathrm{~cm}^{4}$ to $1673 \mathrm{~cm}^{4}$ by $4 \%$ strain. The $I_{X-X} I_{S}$ reduced further with a reduction from $1675 \mathrm{~cm}^{4}$ to $1347 \mathrm{~cm}^{4}$. 
The reduction in the second moment of area in both directions equates to a reduction in the sections capacity to resist bending in both planes. The second moment of area is an important value used in steel design calculations, any change brought about during the bending process must be calculated in order for accurate design calculations to be achieved. The decrease in second moment of area in the $\mathrm{X}-\mathrm{X}$ direction, coupled with the Bauschinger effect will result is a lower required stress to bend the section back in the opposite direction. If the section had been reformed using induction bending the internal stresses caused by bending and the cold-formed production method would be neutralised due to recrystallization. 


\subsection{Chemistry}

It has become increasingly clear that the steel chemistry is one of the most important factors in controlling the properties of the steel tube, both before and after bending. The chemistry of the cold formed tubes has a high CEV which gives it a much higher, unbent hardness, making it more brittle. This means that the cold formed sections are significantly more brittle after bending than the hot-finished and TMRC equivalents. The steel that is used for these sections has a much more lean chemistry with a slightly lower carbon percentage and CEV.

\subsection{Pass Number}

The number of passes that a section makes through the bending apparatus clearly has an effect on the properties of the section. A high pass number yields a larger alteration to the mechanical properties and dimensions of the tubes. When the pass number is reduced, the effect on the mechanical properties and the dimensions is also reduced. However with rate related effects such as wall concavity, a reduced pass number, in most cases increases the severity of the defect. 


\subsection{Spring-back}

The elastic spring-back that occurs after the bending load has been removed is an important consideration. Upon removal of the bending load, the section's deflection will be reduced proportionally to the sections elastic. The severity to which this occurs is based upon the yield strain of the material. The section will always re-straighten by this fixed amount until the UTS has been reached and the material fails. For example the yield strain at the yield point in the hot-finished RHS is approximately $0.176 \%$ so the section will spring-back by $1.32 \mathrm{~mm}$ for a $750 \mathrm{~mm}$ length. This means the section must be over bent in order to reach the target strain. It can be seen from the Strain against distance from the neural axis graphs in the analysis chapter that spring-back has a larger percentage effect on the low strain samples. For example, for the RHS at $0.25 \%$; the pre spring-back strain induced is approximately $0.42 \%$, almost double the target strain. However, the extra strain required for the tighter curvature samples was almost negligible.

\section{Elasto-plastic Deformation}

The sample sections were curved at the extreme fibres to the chosen strains; however, the rest of the section will have experienced a lesser strain rate. The neutral axis will not experience any longitudinal stress or strain. The strain will increase from 0 at the neutral axis to the chosen maximum at the extreme fibres at the extrados and intrados. The tubes undergo elasto-plastic deformation; therefore some area of the cross section will be elastically deformed. However, these areas will not spring-back to their original, unbent position as they are held in position by the plastically deformed areas. This will produce internal stresses within the material. These stresses act in the opposite direction to the bending load to revert to the original, low energy state.

For example, the sample section bent to $0.25 \%$ strain has a zone approximately $40 \mathrm{~mm}$ wide either side of the neutral axis that is held in elastic deformation. This elastic deformation increases as distance from the neutral axis is increased towards the extreme fibres of the section. The elastic deformation increases with distance from the neutral axis until the elastic limit is breached and plastic deformation occurs. As the strain induced is increased, this area of elastic deformation around the neutral axis is reduced. A larger proportion of the section is therefore plastically deformed. If the sample has been completely plastically deformed (plastic hinge) during bending then the section will spring-back uniformly and this residual stress will not be experienced. However, if the section is stressed to this point it would not be fit for purpose.

In cold-bending the loading is cyclical and the sample undergoes many loading and unloading cycles before the required strain is achieved. Work hardening hysteresis should be considered when stress calculation is being performed. Work hardening hysteresis curves often plot the negative stresses, 
however in cold bending the pressure is applied and then removed, it is never reversed therefore the area of the graph where $y \geq 0$ is the relevant part. From the literature it can be seen that the hysteresis loop follows the plastic deformation path of a tensile test, the work hardening can be witnessed from this.

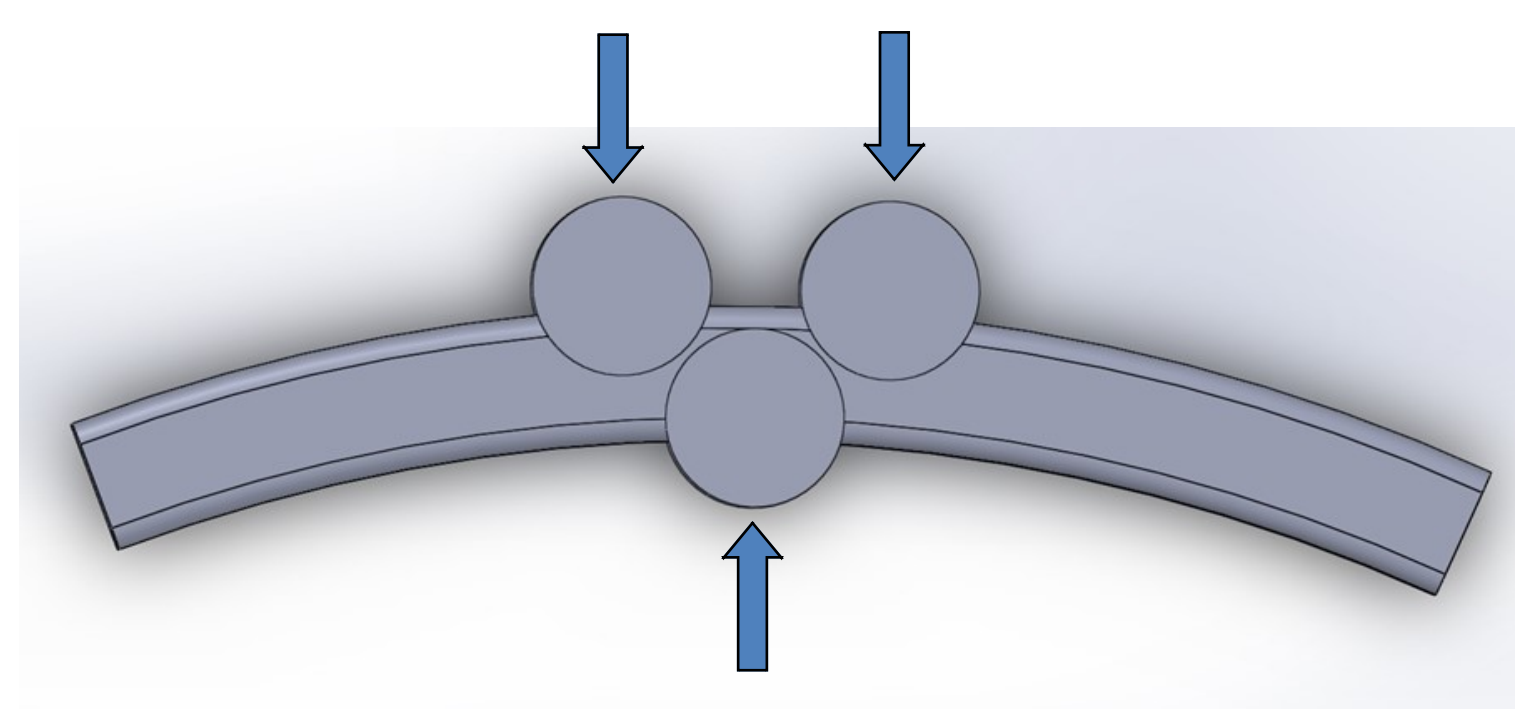

Figure 125-Forming roller force application points after bending 


\subsection{Intrados and Extrados}

The bending moment introduced by the forming rollers causes a compressive force below the neutral axis and a tensile force at the outer edge.

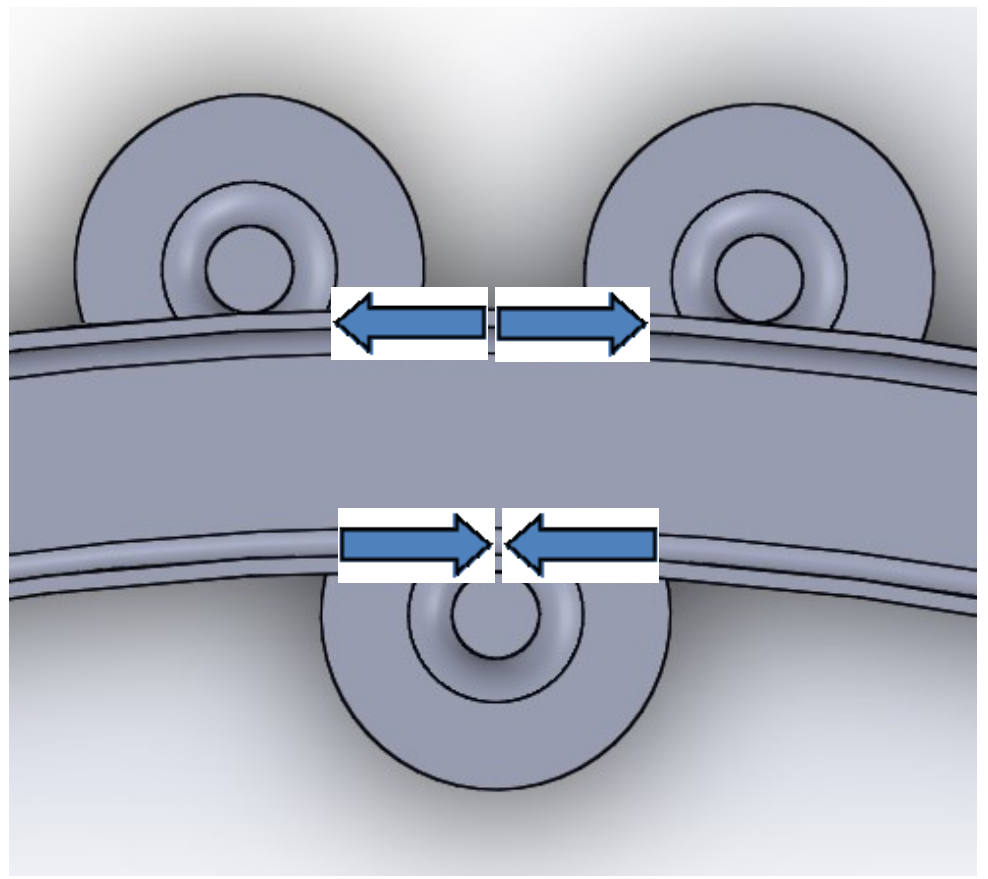

Figure 126-Maximum axial tension and compression zones

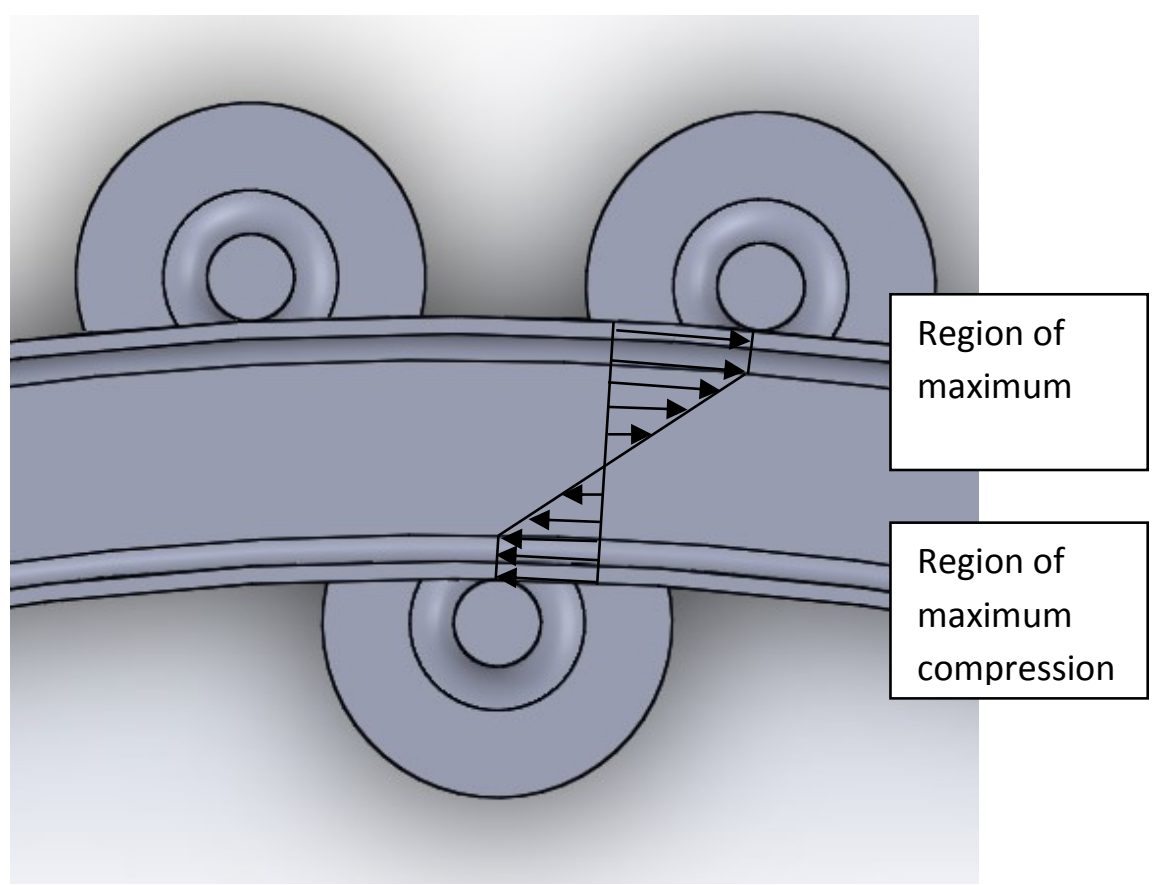

Figure 127-Elastoplastic Stress Distribution 
Figure 126 and Figure 127 show the areas of maximum longitudinal tension and compression and the elasto-plastic stress distribution through a bent section.

The compressive force at the intrados causes material shortening at the inside edge. Due to the conservation of volume, the cross-section will change shape to compensate for this reduction in length. The intrados wall will become thicker, increasing the gauge of the wall. It will also increase in breadth. This change is thickness and breadth is proportional unless the elastic limit of the material has been breached. However, for a material that has been plastically deformed, it is not.

As the strain is increased the corner radii at the intrados are reduced. The material is pressed into the forming rolls transferring the material to the intrados flange. These two factors produce a significant compressive force across the breadth of the intrados causing it to buckle. This reduces the stress on the material. This can be seen in Figure 128, Figure 129 and Figure 130

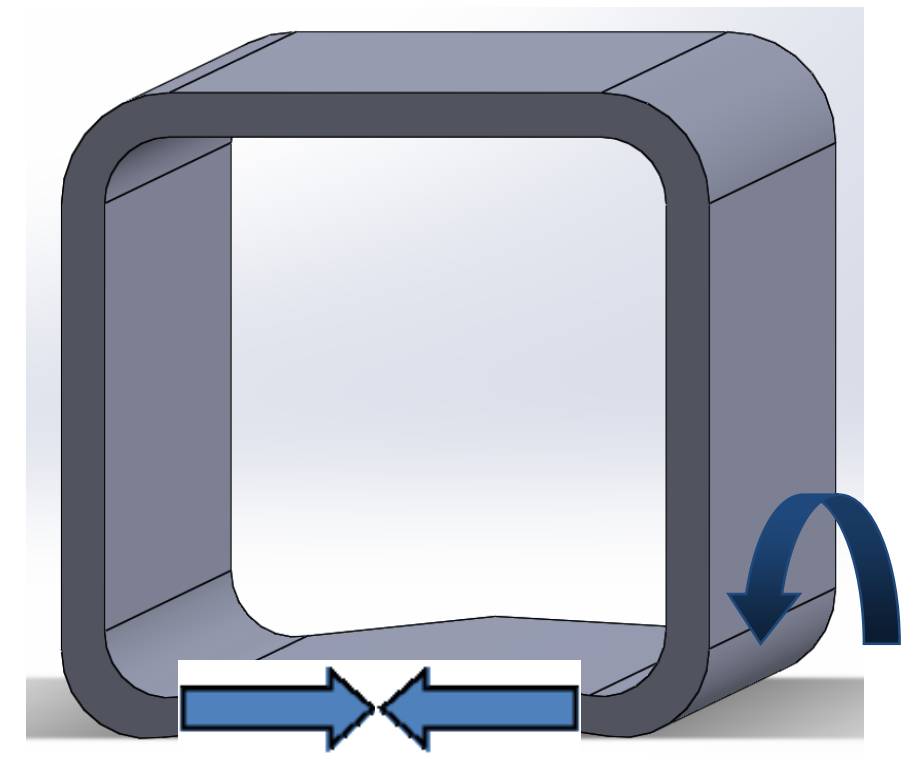

Figure 128-Intrados concavity 


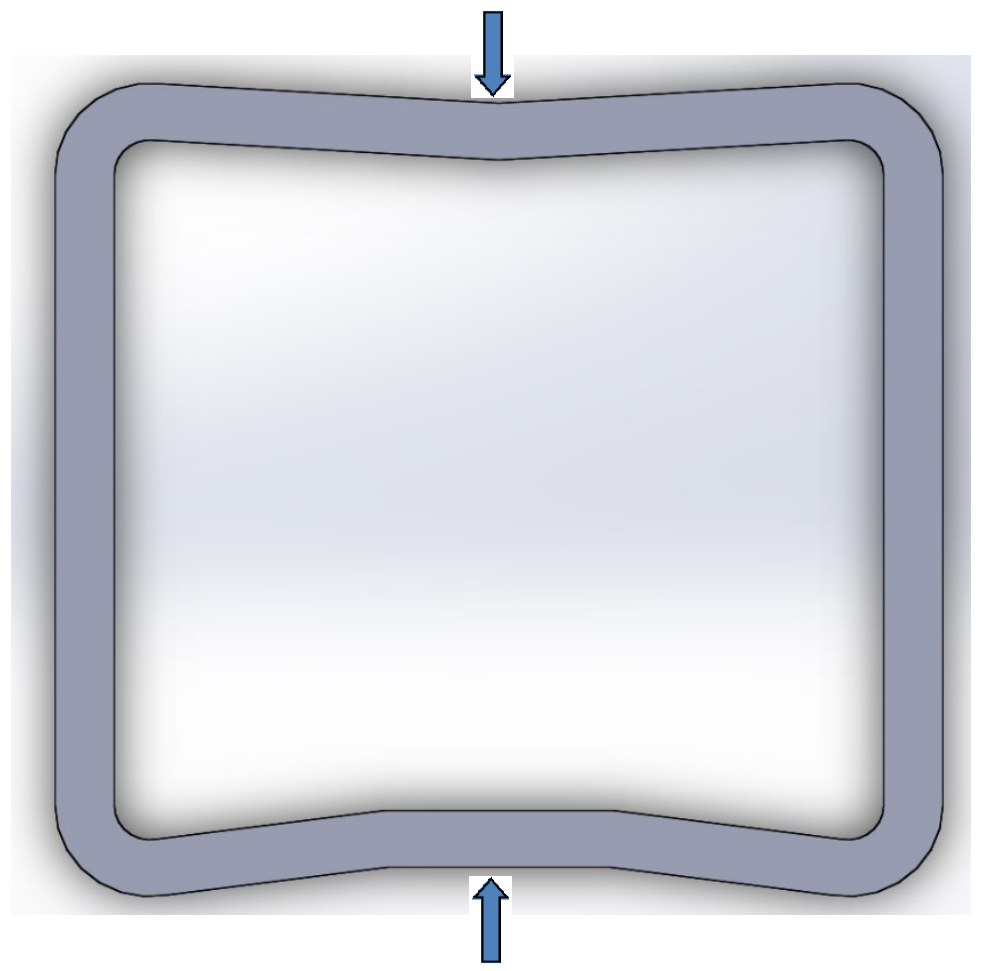

Figure 129-Intrados and Extrados concavity

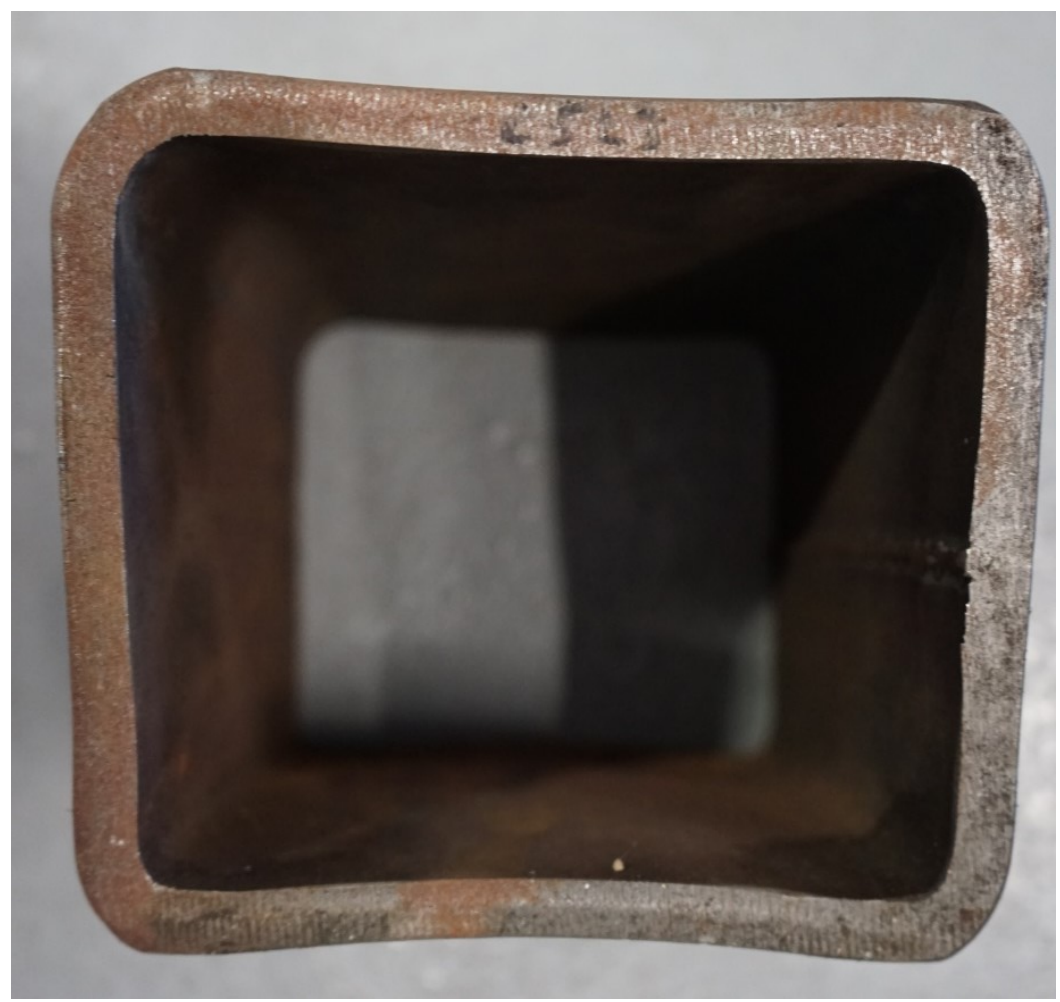

Figure 130-Intrados concavity of cold formed high-strain RHS 
The cause for the collapse of the extrados is less clear; the area is in tension and therefore was expected to experience a reduction in thickness and breadth. However, this was not observed in practice. Extension of a $750 \mathrm{~mm}$ section at the extrados is $37.5 \mathrm{~mm}$ when bent to $5 \%$ stain. Reducing the vertical displacement from the neutral axis reduces the extension required to achieve the same curvature. The section wall will extend by the minimum possible amount in order to reduce internal stresses. This will cause the unsupported wall to bow into the bore to reduce the extension made by the material. With a $4 \mathrm{~mm}$ wall bow the strain is reduced by 0.0027 to 0.0473 . This equates to a reduction in extension of the centre wall of $2 \mathrm{~mm}$ over a $750 \mathrm{~mm}$ bend. The corner sections are too rigid to collapse in and therefore are pinned at the $5 \%$ strain position. A mandrel would cause all of the material (corners and flanges) to extend equally and would prevent this wall collapse from occurring. 


\subsection{Intrados Corners}

The intrados corners are under compression by the forming rollers and the side walls of the section. This will lead to a reduction in the corner radii as the section is pressed into the tighter corner profile of the forming rolls. This will mean that material that was previously part of the corner will be added to the intrados flange. This will contribute to an increased stress across the section breadth and therefore the wall concavity.
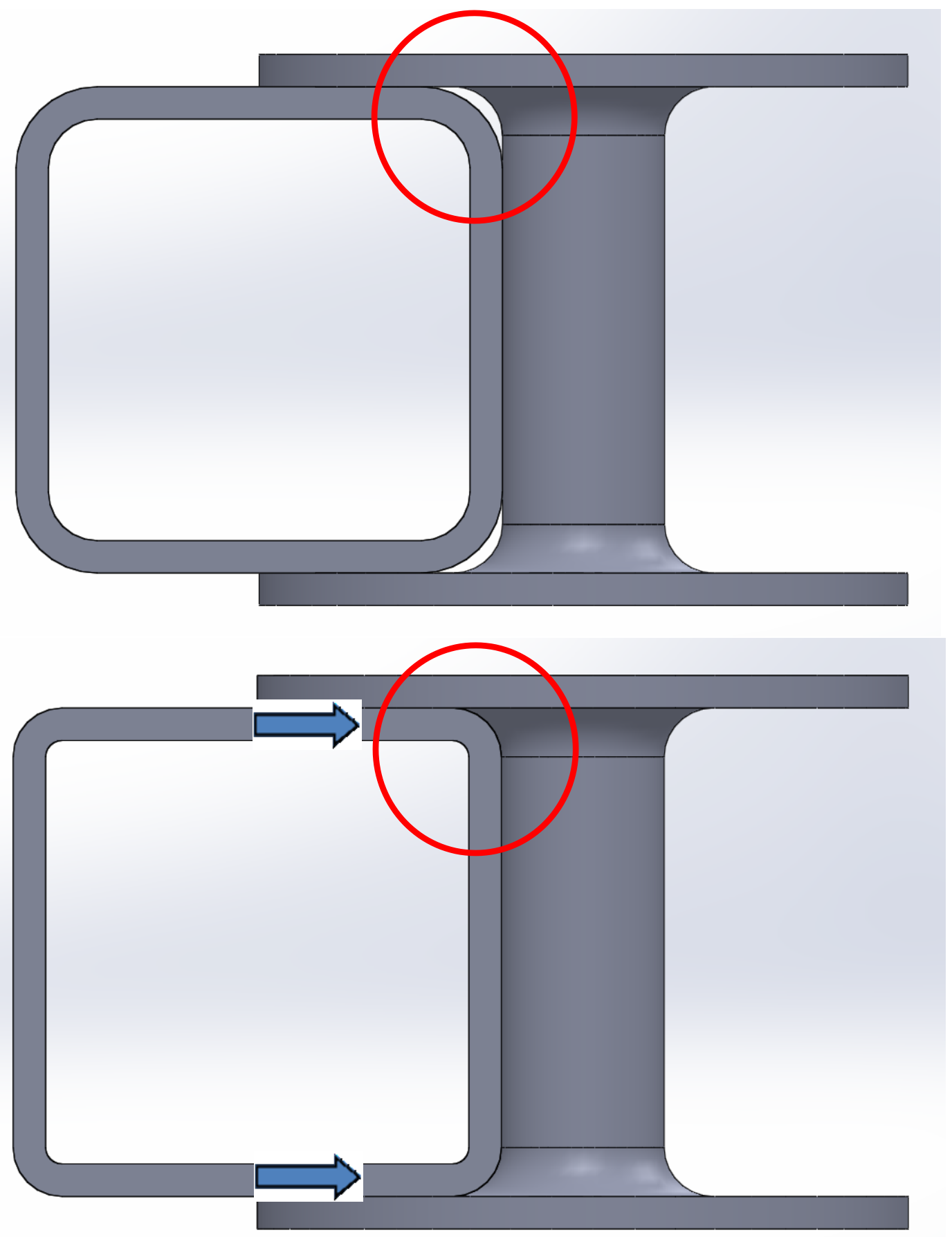

Figure 131-Change at intrados corner radii 
It was originally planned for tensile and Charpy testing to be carried out at the intrados and extrados corners in order to get a true "worst case" stress position. Due to the walls bowing at the intrados and extrados, the flanges are not axially strained to the extent of the corners. However, cutting samples from the corners was deemed too difficult to be achieved. Only microstructure and hardness testing was performed on the corners of the sections. From this testing it can be seen that the corners are area of particular importance and should be focused on in any further work. 


\subsection{Neutral Axis}

Ideally, the neutral axis is the line at which no net force is experienced and therefore there will be no relative change in length. Either side of this line, the material is either in tension or compression. The closer to the extrados, the higher the tension stress conversely, the closer to the intrados, the higher the compressive stress.

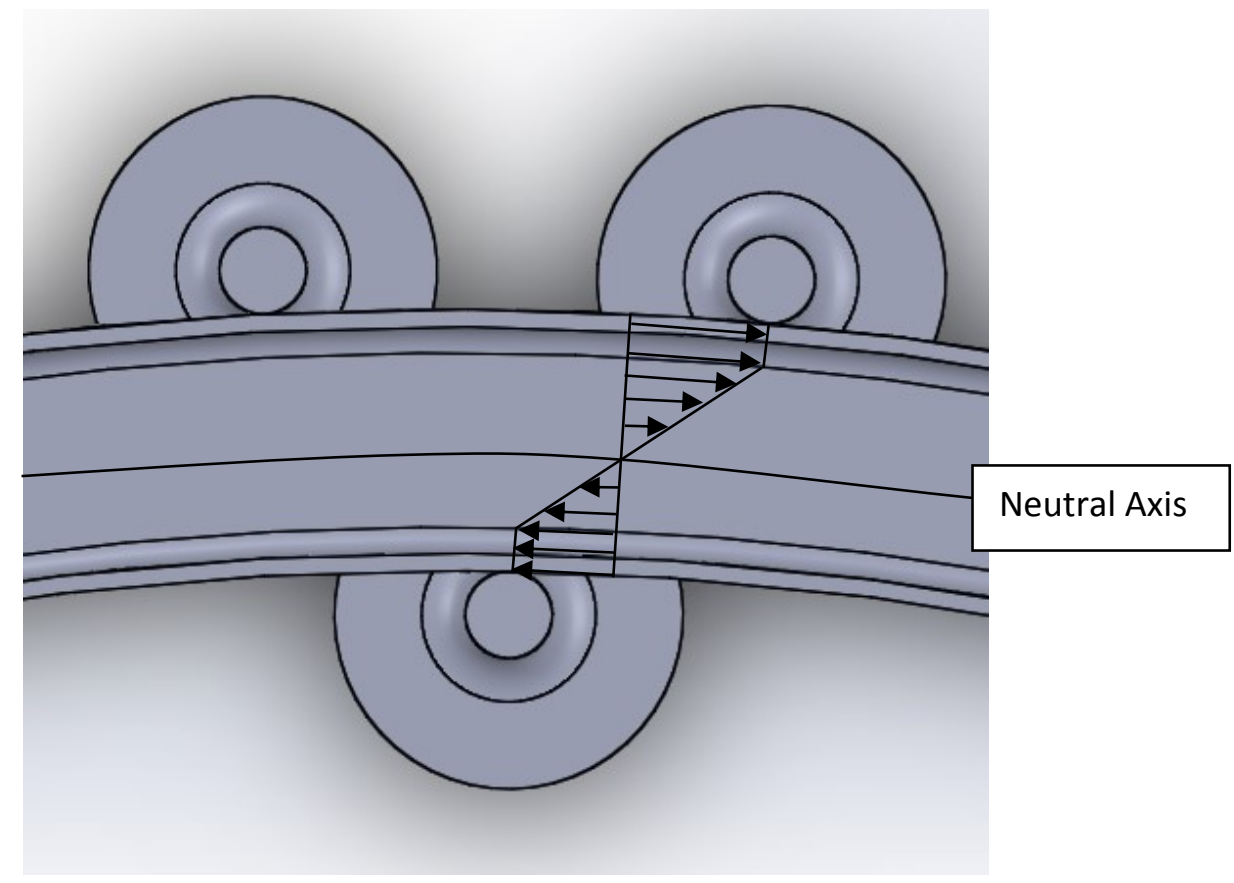

Figure 133-Elastoplastic stress distribution around the neutral axis

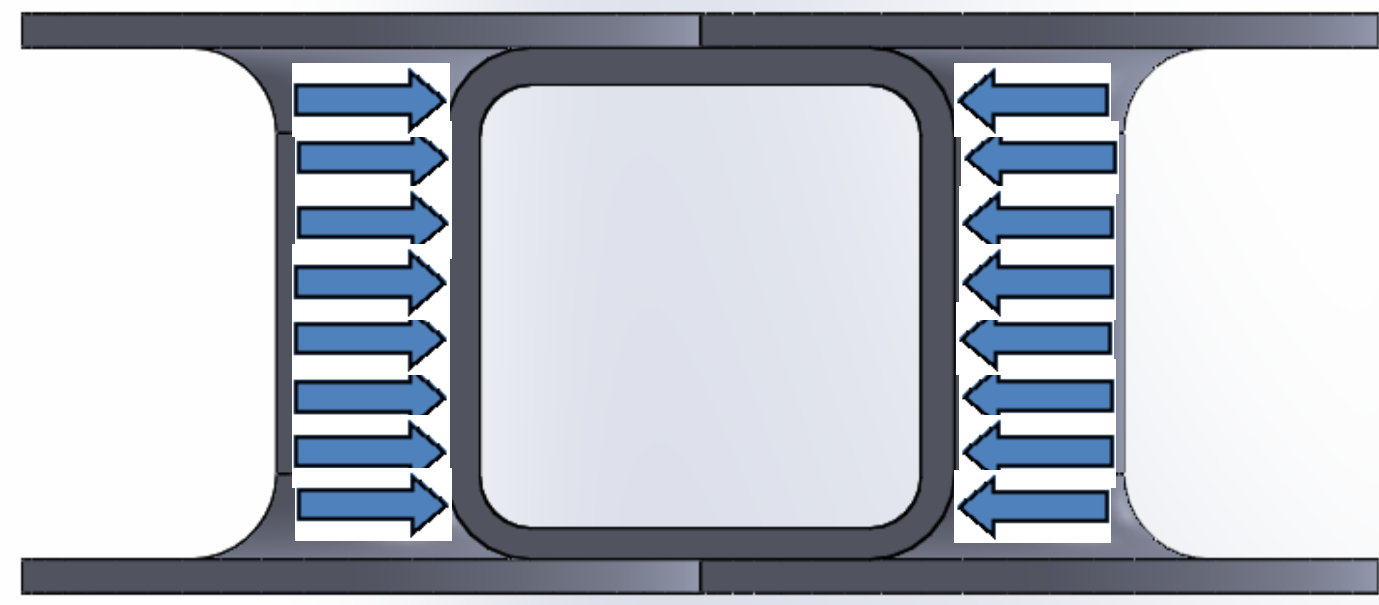

Figure 134-lateral pressure experienced by the RHS during bending 


\subsection{Bauschinger Effect}

The Bauschinger effect is a significant factor that must be considered when cold-roller bending steel sections. The reduction in yield strength that occurs must be taken into account during engineering design calculations. In a number of cases, large reductions in proof stress were recorded. This caused the sections to fail to meet specification requirements. There is no evidence from the testing performed that the strain rate applied affects the severity of the phenomenon. However, the more of the tube cross section that has been yielded, the more area that will be affected by it.

For the low strain bends, a large section of the tube side walls will not have been plastically deformed and are therefore unlikely to be affected by the phenomenon. For the high strain sections, the majority of the section will have been deformed plastically, meaning a larger area of the cross-section will be affected when the loading is reversed. This reduction in yield strength must be compensated for if the sections expected loading will be opposite to that during bending.

The Bauschinger effect is a common phenomenon experienced when steel of any cross-section is manipulated at ambient temperature. It is not exclusive to hollow sections [22], [59]. The reduction in proof stresses observed at the intrados during testing is typical of any cold-bent steel crosssection of a material in compression. Areas in compression during bending will experience a reduced tensile yield stress; conversely, areas in tension during bending will experience a reduction in compressive yield stress. However, the Bauschinger effect is not limited to the yield stress of a material. It is known to increase the ductile-brittle transition temperature, making a material more brittle at higher temperature [5].

The problems associated with the Bauschinger effect could be negated in 2 ways, either alter the chemistry of the section or perform heat treatments. Altering the chemistry in order to ensure steel meets yield requirements could be done in two ways:[22], [59], [11]: Firstly, stronger sections could be used in order to compensate for the reduction in yield strength. This could cause problems during bending as the material will be harder and therefore more expensive to bend. This in turn would increase the cost per bend. Metallurgy could also be altered in order to reduce the dislocation movement and therefore improve the Bauschinger resistance of a steel. It can be seen from the literature that additions of $\mathrm{Cu}, \mathrm{Ni}, \mathrm{Cr}$ in combination with $\mathrm{Nb}$ and $\mathrm{V}$ into steel containing $0.07 \mathrm{C}$ and $1.2 \% \mathrm{Mn}$ improves the Bauschinger resistance[11]

The last option would be to heat treat the section, returning the mechanical properties to those of the unbent section. This is the most expensive solution as large, full-length furnaces are required. This solution removes the cost benefits of cold-roller bending over hot-induction bending. 
The reduction in strength observed is significant and many sections to fail to meet the S355 requirement. However, during steel construction designs, large safety factors are used in order to compensate for material differences and fabrication errors. It is clear from historical records that steel construction is safe, despite using inaccurate data for the calculations. The safety factors compensate for any reduction in mechanical properties caused by the bending process. However, the safety margin will be reduced and therefore, a further safety factor should be introduced for curved steelwork to compensate. 


\subsection{Statistical Significance}

Originally, the testing schedule included many more replicates which would allow for statistical significance checks to be made. This was not achieved due to the difficulty of testing curved steel samples. More replicates would be required in order to carry out any statistical significance checks. Though it is likely that the findings of this investigation re-occur during repetition of the testing, they are based on the small amount of testing performed and could therefore be anomalous. The testing only investigated hot-finished and cold-formed RHS of a particular cross section so cannot be used to predict any effects on the properties of bending of any other grade or section size with any statistical likelihood.

\subsection{Further Work}

If more work were to be performed, a number of additions should be made to improve the validity of the project. This includes carrying out more replicates of the tests that have been performed in order to produce statistically significant results. Extra testing would also be required to gain a better understanding of the worst-case loading areas at the intrados and extrados corners including tensile and Charpy testing. The Charpy testing that has been carried out surpasses current specification by testing the intrados and extrados. However, it should be improved further by testing the corners and testing at a range of temperatures to discern the brittle-ductile transition temperature of the material. The Bauschinger effect is thought to increase the ductile to brittle transition temperature by as much as $3^{\circ} \mathrm{C}$.[5] Compression testing should be carried out to gain a better understanding of the Bauschinger effect.

It would also be beneficial to test other section sizes to ensure that they are affected in the same way. Other grades are likely to be affected differently by the bending process. The Bauschinger effect is highly chemistry dependant [10].

A more comprehensive mathematical model and simulation should be produced in order to more accurately determine the section stresses during bending. This will give a better understanding of the elasto-plastic deformation and the internal stresses of the material after bending and springback have occurred.

Residual stress analysis should be performed using either the hole-expansion method or an STA. An added benefit of the STA is that it would indicate the required temperature at which the residual stresses are alleviated. 


\section{Conclusions}

From the testing performed it is clear that the cold-roller bending process in its current state affects both the mechanical and geometric properties in many ways. Some of the affects that have been noted are more detrimental than others.

- A moderate increase in the yield strength was observed during tensile testing when the testing is performed in the same direction as bending strain in the material. However, the hot-finished product does not exhibit this increase in yield strength as all of the bending was carried out in the discontinuous yielding zone.

- Conversely, when the testing was carried out at $180^{\circ}$ to the bending strain, the yield strength of the material is significantly reduced. This has been attributed to the Bauschinger effect.

- Further testing revealed that the reduction in yield strength increased with strain applied to the section during bending. This was also shown during simulated bending on the tensile tester with both compression-tension testing and 3 point bend testing.

- The ultimate tensile strength of the section was largely unaffected by the bending process and the results remained largely consistent for both hot-finished and coldformed sections.

- Both hot-finished and cold-formed sections experienced a similar drop in yield when the testing load is $180^{\circ}$ to the bending load however, cold-formed sections start with a higher initial yield strength so do not fail to meet the requirements of S355 steel. The cold-formed product experienced the greatest reduction in yield strength with a reduction of $16 \mathrm{MPa}$ more than the hot-finished product at worst case.

- Further testing on the hot-finished product revealed that the properties are restored after the pre-strain threshold has been breached. This restoration of properties is likely the reason that no structures in the literature are known to have collapsed due to the Bauschinger effect. The large safety factor applied to structures will also allow for a reduction in yield strength.

- Hardness increases with increasing strain for all areas that experienced a net change in length. The average increase in hardness for the hot-finished product for the extrados was $15 \mathrm{HV}$ with an increase of $30 \mathrm{HV}$ at the corners. The cold-formed product experienced smaller increases in hardness as the material was already cold worked so had already experienced an increase in hardness. 
- It is possible that the increase in hardness would be greater if the hardness testing was performed orthogonal to the direction of axial displacement.

- Neutral axis hardness is not altered by the bending process as there is no net change in length. Neither the hot-finished or the cold-formed product experienced an increase in hardness or yield strength at the neutral axis.

- The consistency of the results for both products, before and after bending suggests that testing, typically performed at the neutral axis is not sufficient to assess the quality of the section after bending has been performed.

- The Charpy impact testing performed suggested that the hot-finished product was better suited to cold-roller bending. The cold-formed product exhibited significantly lower and less consistent impact energies than the hot-finished product.

- Most of the sample cross-sections failed to meet the geometric requirements for their straight standards (BS EN 10219-2:2006 for cold-formed and BS EN 102102:2006 for hot-finished[7], [9], [55], [56]). Even at relatively low strains, the H:D ratio was the cause of the failure in the majority of samples. The samples became too narrow across the $\mathrm{X}$-X direction as the section was compressed. The cold-formed sections performed poorly in this aspect with the out of square ratio greater than $6 \%$ by $1 \%$ strain. The maximum value was greater than $11 \%$. None of the hotfinished sections surpassed $9 \%$.

- At higher strains, both the intrados and extrados faces buckled. Wall concavity reduces the strain in that area by reducing the distance from the neutral axis and therefore the extension or compression of the material in that area.

- The corners of the sections are stiffer due to their geometry and do not buckle in the same way as the flat faces at the intrados and extrados. It is therefore likely that the corners are the worst-case areas for extension or compression.

- This assumption makes testing at the corners increasingly important for assessing the true effects on bending on the properties of the sections. Impact and tensile testing must be carried out at the corners to truly asses these changes. Hardness testing can be used to calculate the yield strength[60] and the ultimate tensile strength of a material [61], [62].

- Largely it is this reduction in the X-X outer dimension that causes the changes in the geometric properties. If this reduction in section depth could be minimised it would significantly reduce the effect of bending of the geometric properties of the crosssection. 
- The second moment of area of both the hot-finished and cold-formed sections is reduced in the X-X direction, thus its resistance to bending is reduced. This is caused by the deformation of the cross-section, specifically, the reduction in section depth caused by the compression of the section exerted by the bending rollers. 


\section{References}

[1] T. J. Wilkinson, "The plastic behaviour of cold-formed rectangular hollow sections," no. October, 1999.

[2] D. Brown, "Design of Curved Steel," Steel Constr. Inst., 2007.

[3] J. Rondal, K.-G. Würker, D. Dutta, J. Wardenier, N. Yeomans, B. Standard, P. Description, and M. stein S.B. Batdorf , Murry Schildcrout, Structural Stability of Hollow Sections, vol. 3, no. 1. 1989.

[4] B. E. 1090-1:2009, Execution of steel structures and aluminium structures Part 1: Requirements for conformity assessment of structural components. 2009, pp. 1-46.

[5] A. C. Bannister, M. Skalidakis, A. Pariser, P. Langenberg, F. G.-S. Salcedo, L. Sanchez, D. Pesquera, and W. Azpiazu, "Performance Criteria for Cold-formed Structural Steels," 2003.

[6] T. Wilkinson and G. J. Hancock, "Compact or Class 1 Limits for Rectangular Hollow Sections in Bending," pp. 1-8, 1998.

[7] B. E. 10219-2:2006, "Cold formed welded structural hollow sections of non-alloy and fine grain steels - Part 2: Tolerances, dimensions and sectional properties," Eur. Stand., vol. 3, no. February, 2006.

[8] J. Wang, S. Afshan, M. Gkantou, M. Theofanous, C. Baniotopoulous, and L. Gardner, "Flexural behaviour of hot-finished high strength steel square and rectangular hollow sections," J. Constr. Steel Res., vol. 121, pp. 97-109, Jun. 2016.

[9] B. E. 10219-1:2006, "Cold formed welded structural hollow sections of non-alloy and fine grain steels - Part 1: Technical delivery conditions," Eur. Stand., vol. 3, no. February, 2006.

[10] A. G. Kostryzhev, "BAUSCHINGER EFFECT IN Nb AND V MICROALLOYED LINE PIPE STEELS by Andrii Gennadiovych Kostryzhev A thesis submitted to The University of Birmingham for the degree of DOCTOR OF PHILOSOPHY School of Metallurgy and Materials College of Engineering and Physical," University of Birmingham, 2009.

[11] H. I. Bharata, A. Desrianto, and Z. A. Muslim, "The Development of Hot Rolled Coil of API5LX65 Specifications With Improved Bauschinger Effec Resistance for Oil and Gas Linepipe Applications," SEAISI, vol. 40, no. 3, pp. 37-42, 2011.

[12] B. Crouch, "WHAT PIPELINERS NEED TO KNOW ABOUT INDUCTION BENDS," Melbourne.

[13] H. a. Al-Qureshi, "Elastic-plastic analysis of tube bending," Int. J. Mach. Tools Manuf., vol. 39, no. 1, pp. 87-104, 1999.

[14] W. L. Costin, O. Lavigne, and A. Kotousov, "Materials Science \& Engineering A A study on the relationship between microstructure and mechanical properties of acicular ferrite and upper bainite," Mater. Sci. Eng. A, vol. 663, pp. 193-203, 2016. 
[15] T. Ono, Y. Tomota, P. Lukas, D. Lugovy, D. Neov, N. Tsuchida, and K. Nagai, "In situ neutron diffraction during tensile straining of fine grained ferrite-pearlite steel," Mater. Sci. Technol., vol. 20, pp. 121-125, 2004.

[16] J. Richards, "A Mechanical and Microstructural Comparison of Induction Bent, Welded and Seamless Plain Carbon Steel Tubes," Swansea University, 2013.

[17] X. P. Huang and W. C. Cui, "Effect of Bauschinger Effect and Yield Criterion on Residual Stress Distribution of Autofrettaged Tube," J. Press. Vessel Technol., vol. 128, no. 2, p. 212, 2006.

[18] F. Yoshida, T. Uemori, and K. Fujiwara, "Elastic-plastic behavior of steel sheets under in-plane cyclic tension-compression at large strain," Int. J. Plast., vol. 18, no. 5-6, pp. 633-659, 2002.

[19] J. Gau and G. L. Kinzel, "A new model for springback prediction in which the Bauschinger e ect is considered," vol. 43, pp. 1813-1832, 2001.

[20] A. K. Roy, S. Bandyopadhyay, S. B. Suresh, D. Maitra, P. Kumar, D.Wells, and L. Ma, "Relationship of residual stress to dislocation density in cold-worked martensitic alloy," Mater. Sci. Eng. A, vol. 416, pp. 134-138, 2006.

[21] M. Zhan, H. Yang, L. Huang, and R. Gu, "Springback analysis of numerical control bending of thin-walled tube using numerical-analytic method," J. Mater. Process. Technol., vol. 177, no. 1-3, pp. 197-201, 2006.

[22] R. C. Spoorenberg, H. H. Snijder, and J. C. D. Hoenderkamp, "Mechanical properties of roller bent wide flange sections - Part 2: Prediction model," J. Constr. Steel Res., vol. 68, no. 1, pp. 63-77, 2012.

[23] J. Shackelford, Introduction to materials science for engineers., 3rd ed. 1992.

[24] W. D. Callister and D. G. Rethwisch, Materials Science and Engineering: An Introduction, 8th ed. J.W.Wiley \& Sons, Inc, 2009.

[25] X. Wang and J. Cao, "Wrinkling Limit in Tube Bending," J. Eng. Mater. Technol., vol. 123, no. 4, p. 430, 2001.

[26] L. Heng, Y. He, Z. Mei, S. Zhichao, and G. Ruijie, "Role of mandrel in NC precision bending process of thin-walled tube," Int. J. Mach. Tools Manuf., vol. 47, no. 7-8, pp. 1164-1175, 2007.

[27] E. Muthmann and F. Grimpe, "Fabrication of hot induction bends from LSAW large diameter pipes manufactured from TMCP plate FABRICATION OF HOT INDUCTION BENDS FROM LSAW LARGE," in Microalloyed Steels for the Oil \& Gas Industry International Symposium, 2006.

[28] E. Muthmann, W. Kaluza, M. Liedke, and W. Scheller, "Induction bends in material Grade X80 : experience from more than 15 years," in Pipeline Technology Conference, 2009, no. October. 
[29] H. Lee, J. Bae, M. Kim, and C. Kim, “Optimum Design of Pipe Bending Based on HighFrequency Induction Heating Using Dynamic Reverse Moment," Precis. Eng. Manuf., vol. 12, no. 6, pp. 1051-1058, 2011.

[30] V. I. Rudnev, "A common misassumption in induction hardening," Heat Treat. prgress, no. October, pp. 23-25, 2004.

[31] R. Naar and F. Bay, "Numerical optimisation for induction heat treatment processes," Appl. Math. Model., vol. 37, pp. 2074-2085, 2013.

[32] R. T. Alexandre, "Induction Bending of Internally Clad Steel Pipes: Failure Mechanisms \& Processing Parameter Optimization in Ni-base Alloy Weld Overlays," 2016.

[33] E. Kita, S. Hashimoto, T. Kayano, M. Minagawa, H. Yanagihara, M. Kishimoto, T. Oda, N. Ohkohchi, T. Takagi, T. Kanamori, Y. Ikehata, E. Kita, S. Hashimoto, T. Kayano, M. Minagawa, and H. Yanagihara, "Heating characteristics of ferromagnetic iron oxide nanoparticles for magnetic hyperthermia Heating characteristics of ferromagnetic iron oxide nanoparticles for magnetic hyperthermia," Jounal Appl. Phisics, vol. 123902, no. 2012, pp. 8-11, 2016.

[34] P. Jankejech, P. Fabian, J. Broncek, and Y. Shalapko, "INFLUENCE OF TEMPERING ON MECHANICAL PROPERTIES OF INDUCTION BENT S BELOW $540^{\circ} \mathrm{C}$," Acta Mech. Autom., vol. 10, no. 2, pp. 81-86, 2016.

[35] W. F. Smith and J. Hashemi, Foundation of Materials Science and Engineering, 5th ed. Mc Graw Hill, 2011.

[36] M. H. Cai, S. S. Dhinwal, Q. H. Han, Q. Chao, and P. D. Hodgson, "Gradient ultrafine ferrite and martensite structure and its tensile properties by asymmetric rolling in low carbon microalloyed steel," Mater. Sci. Eng. A, vol. 583, no. July, pp. 205-209, 2014.

[37] P. J. McNally, "Techniques: 3D imaging of crystal defects," Nature, vol. 496, no. 7443, pp. 37$38,2013$.

[38] W. . Read, Dislocations in crystals. 1953.

[39] A. Hadhud, "Design of Reducing Agent for Sintering of High - Performance Alloyed PM Steels Based on Different Carbon Grades Analysis by Aimn Hadhud (c) Aimn Hadhud 2012 Department of Materials and Manufacturing Technology," no. 95, 2012.

[40] J. K. Mahato, P. S. De, A. Sarkar, A. Kundu, and P. C. Chakraborti, "Effect of deformation mode and grain size on Bauschinger behavior of annealed copper," Int. J. Fatigue, vol. 83, pp. 42$52,2015$.

[41] D.-I. K.-H. Brensing and D.-I. B. Sommer, "Steel Tube and Pipe Manufacturing Processes Steel Tube and Pipe Manufacturing Processes."

[42] A. P. Parker and J. H. Underwood, "TECHNICAL REPORT ARCCB-TR-98010 THE BAUSCHINGER 
EFFECT IN AUTOFRETTAGED TUBES - A COMPARISON OF MODELS INCLUDING THE ASME CODE BENET LABORATORIES," 1998.

[43] "Effect of Stress reversal on the work hardening behaviour of polycrystalline copper," Acta Metall., vol. 34, no. 8, pp. 1553-1562, 1986.

[44] E. F. Rauch and J.-H. Schmitt, "Dislocation substructures in mild steel deformed in simple shear," Mater. Sci. Eng. A, vol. 113, pp. 441-448, 1989.

[45] E. F. Rauch, J. J. Gracio, F. Barlat, A. B. Lopes, and J. F. Duarte, "Hardening behaviour and structural evolution upon strain reversal of aluminium alloys," Scr. Mater., vol. 46, pp. 881886, 2002.

[46] A. Abel and H. Muir, "The Bauschinger effect and discontinuous yielding," Philos. Mag., vol. 26, pp. 489-504, 1972.

[47] D. V. Wilson and P. S. Bate, "Bauschinger effects in strain aged steels," Mater. Forum, vol. 10, no. 1, pp. 33-42, 1987.

[48] A. Abel, "Historical perspectives and some of the main features of the Bauschinger effect," Mater. Forum, vol. 10, no. 1, pp. 11-26, 1987.

[49] G. Vincze, E. F. Rauch, J. J. Gracio, F. Barlat, and A. B. Lopes, "A comparison of the mechanical behaviour of an AA1050 and low carbon steel deformed upon strain reversal," Acta Meterialia, vol. 53, pp. 1005-1013, 2005.

[50] K. Terada, K. Matsui, M. Akiyama, and T. Kuboki, "Mumerical re-examination of the microscale mechanism of the Bauschinger effect in carbon steel," Comput. Mater. Sci., vol. 31, pp. 67-83, 2004.

[51] R. C. Spoorenberg, H. H. Snijder, J. C. D. Hoenderkamp, and D. Beg, “Design rules for out-ofplane stability of roller bent steel arches with FEM," J. Constr. Steel Res., vol. 79, pp. 9-21, 2012.

[52] B. K. Chun, H. Y. Kim, and J. K. Lee, "Modeling the Bauschinger effect for sheet metals, part II: applications," Int. J. Plast., vol. 18, no. 5-6, pp. 597-616, 2002.

[53] F. Yoshida and T. Uemori, "A model of large-strain cyclic plasticity describing the Bauschinger effect and workhardening stagnation," in International Journal of Plasticity, 2002.

[54] S. P. Committee, E. Effects, S. Preparation, H. R. Innovation, and M. I. Standards, "Carbon Equivalent Limits for Thick Carbon and Low Alloy Steels," 2000.

[55] B. E. 10210-1:2006, "Hot finished structural hollow sections of non-alloy and fine grain steels - Part 1: Technical delivery conditions," Eur. Stand., vol. 3, no. February, 2006.

[56] B. E. 10210-2:2006, "Hot finished structural hollow sections of non-alloy and fine grain steels - Part 2: Tolerances, dimensions and sectional properties," Eur. Stand., vol. 3, no. February, 
2006.

[57] "Effect of strain and strain rate on the Bauschinger effect response of three different steels," Metall. Mater. Trans. A, vol. 36, pp. 2379-2384, 2005.

[58] N. C. Tang, "Plastic-deformation analysis in tube bending," Int. J. Press. Vessel. Pip., vol. 77, no. 12 , pp. 751-759, 2000.

[59] R. C. Spoorenberg, H. H. Snijder, and J. C. D. Hoenderkamp, "Mechanical properties of roller bent wide fl ange sections - Part 1 : Experimental investigation," Jcsr, vol. 68, no. 1, pp. 51$62,2012$.

[60] P. Leroux, "An indentation test that measures yield strength," Adv. Mater. Process., vol. 169, no. 10, pp. 34-35, 2011.

[61] M. Gasko and G. Rosenberg, "Correlation Between Hardness and Tensile Properties in UltraHigh Strength Dual Phase Steels-Short Communication," Mater. Eng. ..., vol. 18, pp. 155-159, 2011.

[62] M. Tiryakioğlu, J. S. Robinson, M. A. Salazar-Guapuriche, Y. Y. Zhao, and P. D. Eason, "Hardness-strength relationships in the aluminum alloy 7010," Mater. Sci. Eng. A, vol. 631, pp. 196-200, 2015.

[63] "Linear Defects - Dislocations." [Online]. Available: https://www.ndeed.org/EducationResources/CommunityCollege/Materials/Structure/linear_defects.htm. 